\title{
Shocks, Insurance and Welfare Evidence from Field Experiments in Ethiopia
}

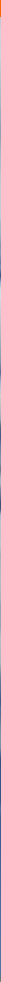

(1)

Desprosent

Nenowish

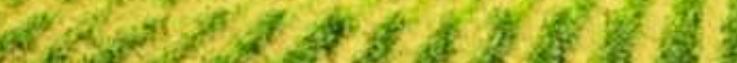

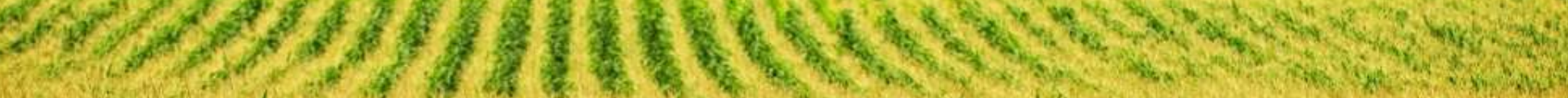

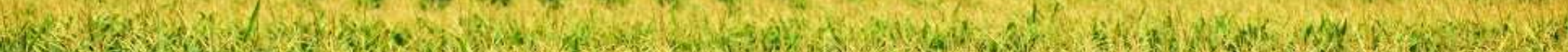
17 3.

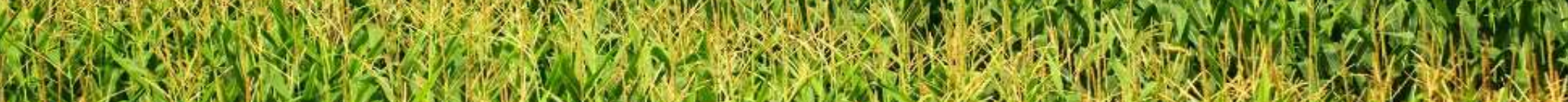
7. 2. (2)

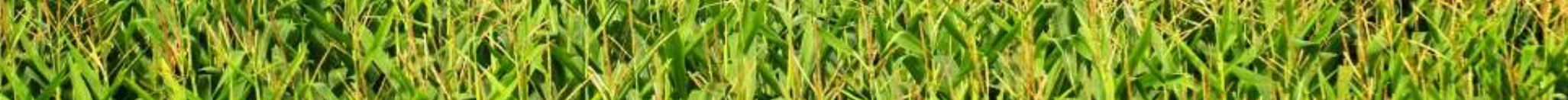
W N

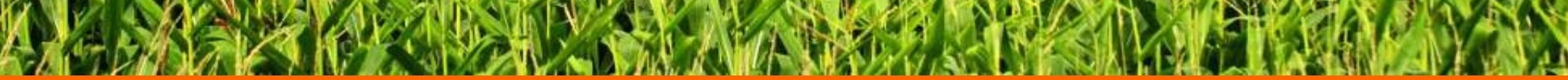


Shocks, Insurance and Welfare Evidence from Field Experiments in Ethiopia

Temesgen Keno Belissa 


\section{Thesis committee}

\section{Promotor}

Prof. Dr. B.W. Lensink

Special Professor, Finance and Development

Wageningen University and Research

\section{Co-promotor}

Dr. M.A.P.M. van Asseldonk

Senior Researcher, Development Economics Group

Wageningen University and Research

\section{Other members}

Prof. M.P.M. Meuwissen, Wageningen University and Research

Dr. Cornelis Gardebroek, Wageningen University and Research

Prof. C.L.M. Hermes, University of Groningen

Prof. I. Soumaré, Laval University, Canada

This research was conducted under the auspices of the Wageningen School of Social Sciences (WASS) 


\section{Shocks, Insurance and Welfare Evidence from Field Experiments in Ethiopia}

Temesgen Keno Belissa

Thesis

submitted in fulfilment of the requirements for the degree of doctor at Wageningen University by authority of the Rector Magnificus, Prof. Dr A.P.J. Mol, in the presence of the

Thesis Committee appointed by the Academic Board to be defended in public on Tuesday 12 March 2019 at 13.30 in the Aula. 
Temesgen Keno Belissa

Shocks, Insurance and Welfare: Evidence from Field Experiments in Ethiopia, 172 pages

$\mathrm{PhD}$ Thesis, Wageningen University, Wageningen, the Netherlands (2019)

With references, with summary

ISBN 978-94-6343-592-5

DOI: https://doi.org/10.18174/470355 
To my late father Keno Belissa Bosona (1946 - 2011) and my late mother Idoshe Bedassa Tullu (1952 - 2013) 


\section{Table of contents}

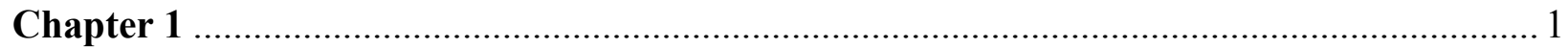

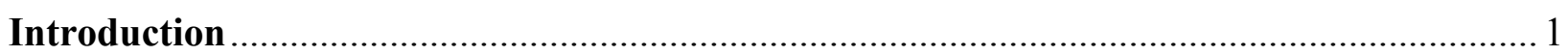

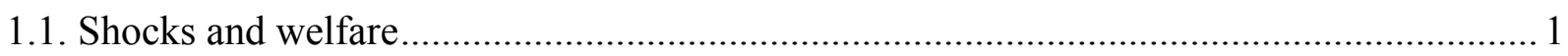

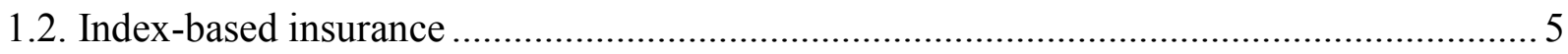

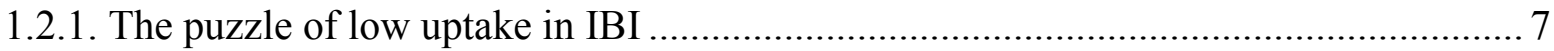

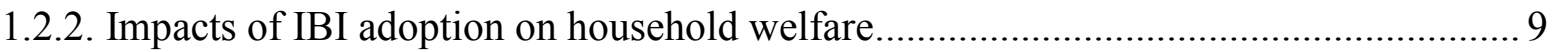

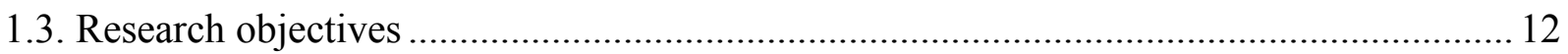

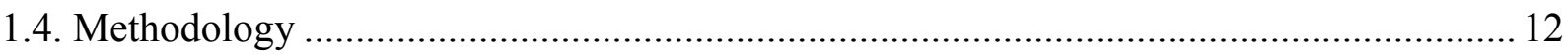

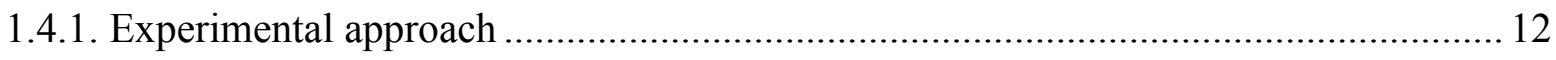

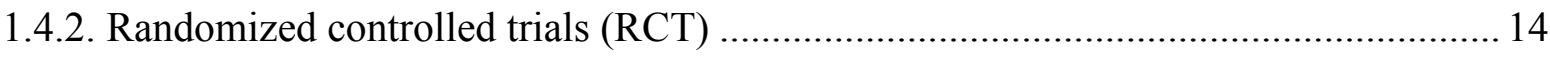

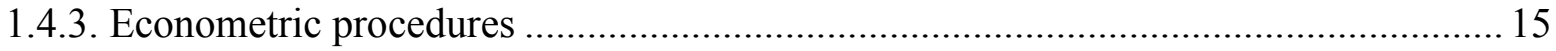

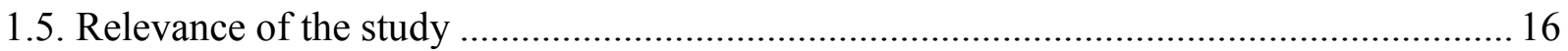

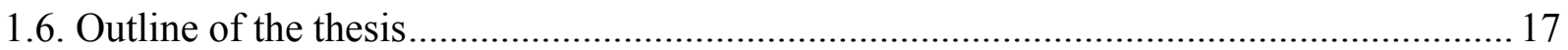

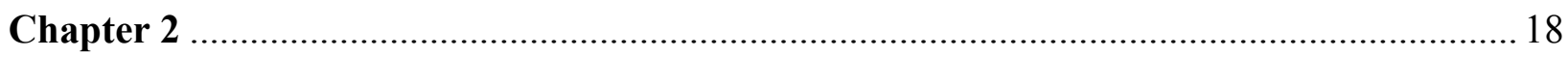

Risk and ambiguity aversion behaviour in index-based insurance uptake decision:

Experimental evidence from Ethiopia........................................................................... 18

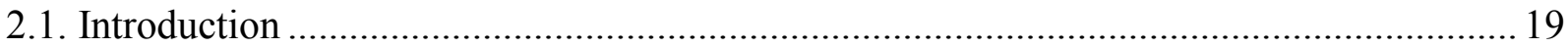

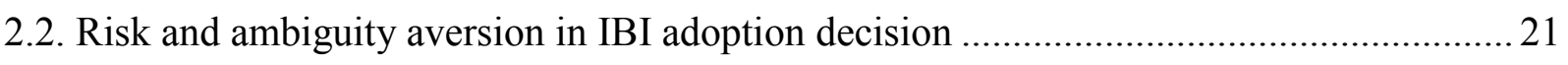

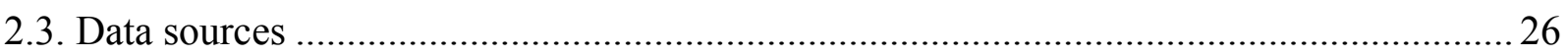

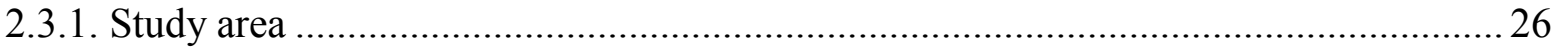

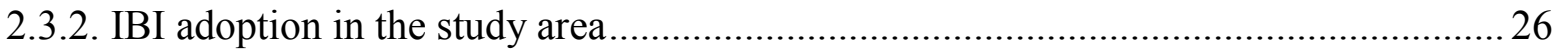

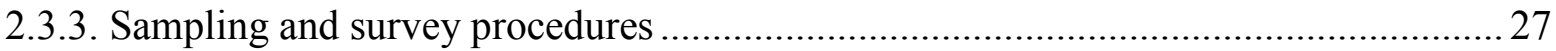

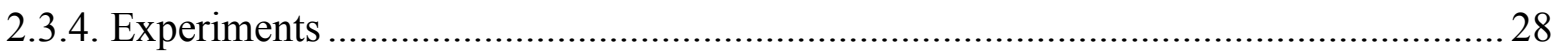

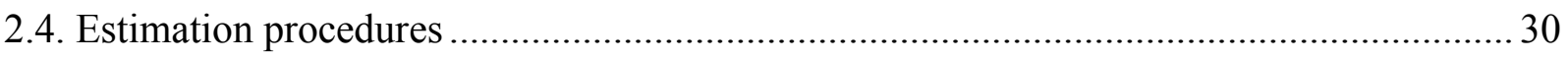

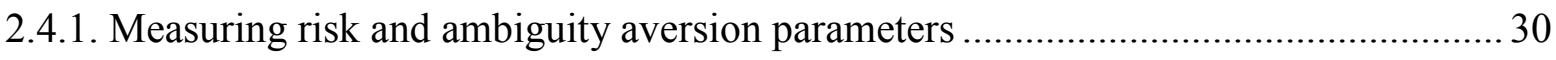

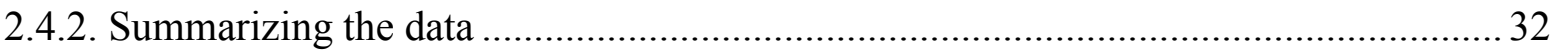

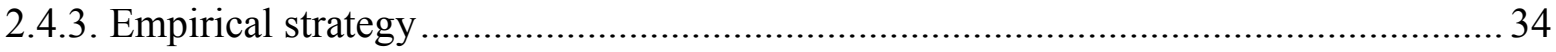

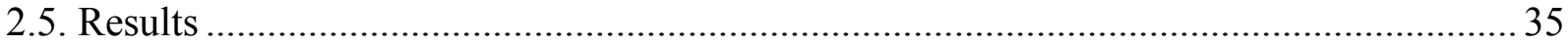

2.5.1. Impacts of risk and ambiguity aversion on the probability of IBI adoption................ 35

2.5.2. Impacts of risk and ambiguity aversion on intensity of IBI adoption ........................ 37

2.5.3. Impacts of risk and ambiguity aversion on late, dropout and persistent IBI adoption

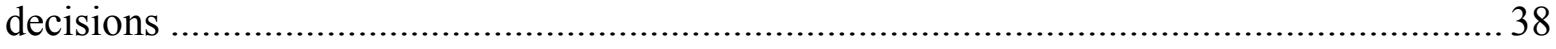

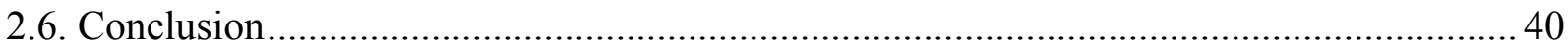


Trust, liquidity constraints and the adoption of weather insurance: A randomized controlled trial in Ethiopia.

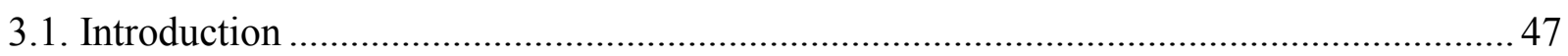

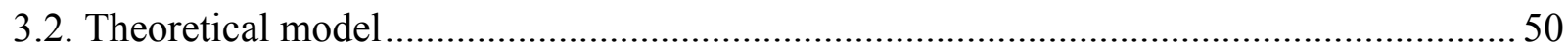

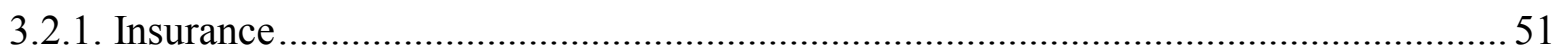

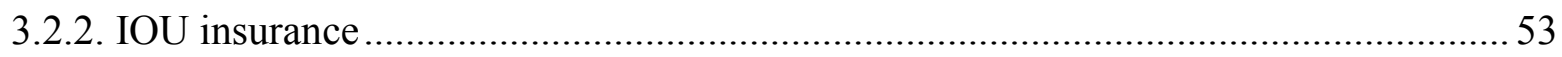

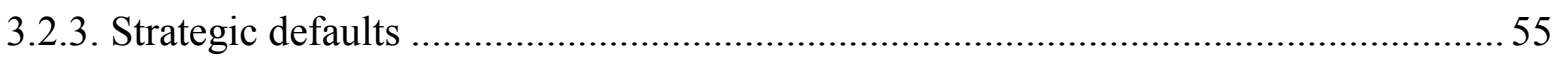

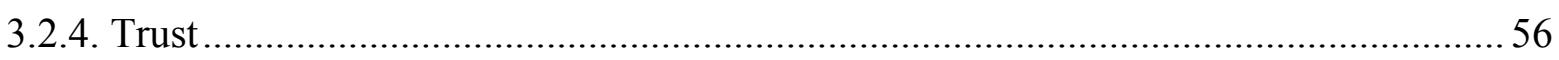

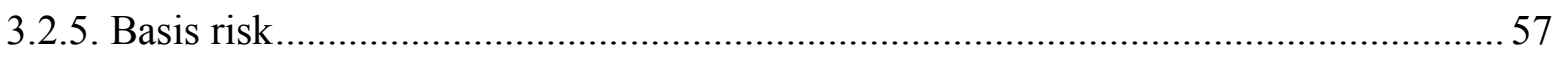

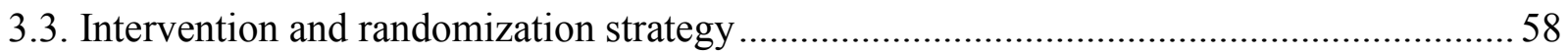

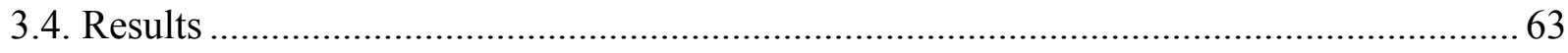

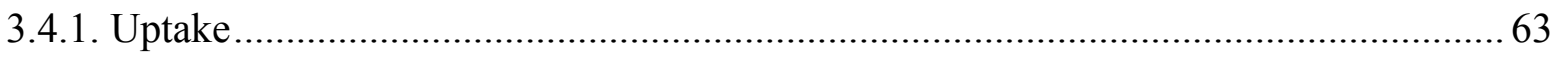

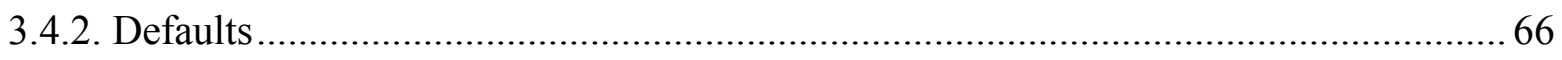

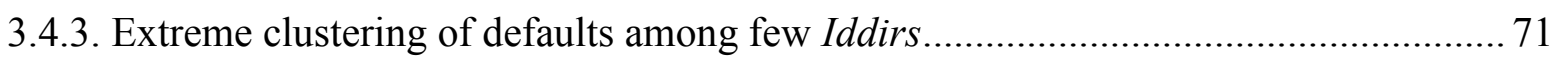

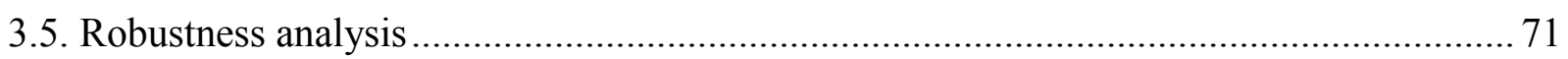

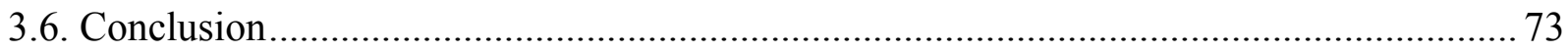

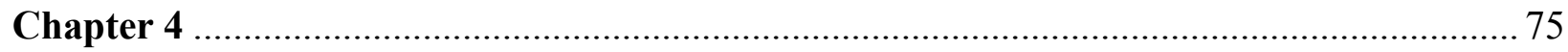

Effects of index insurance on demand and supply of credit: Evidence from Ethiopia ........ 75

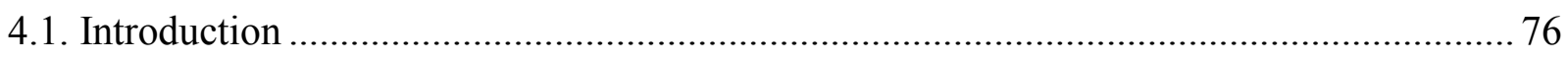

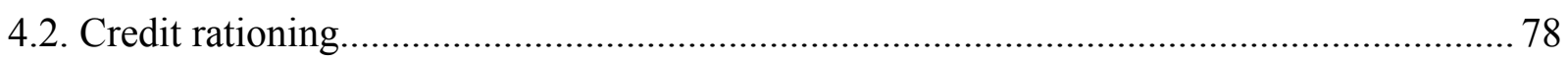

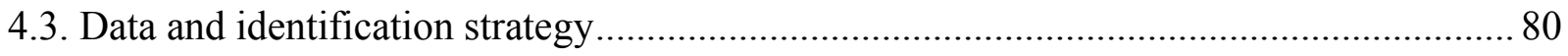

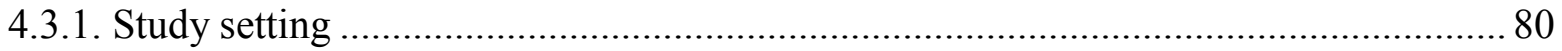

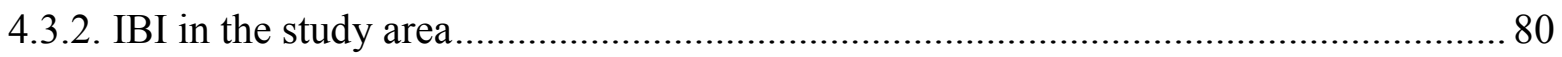

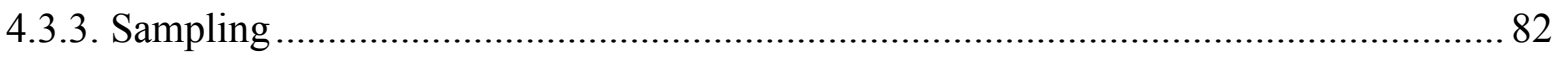

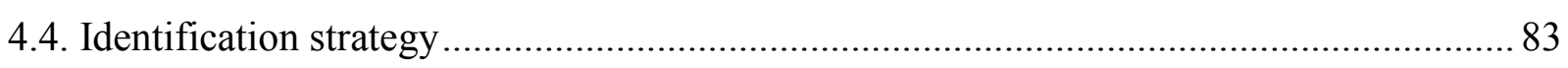

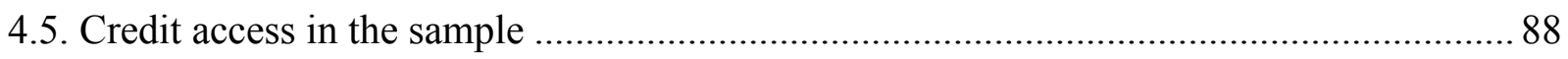

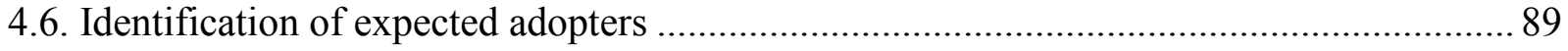

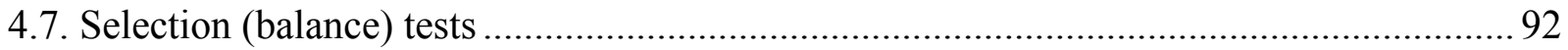

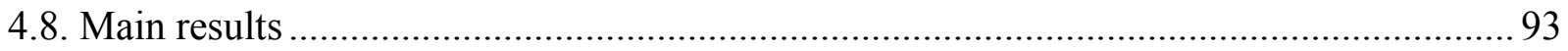

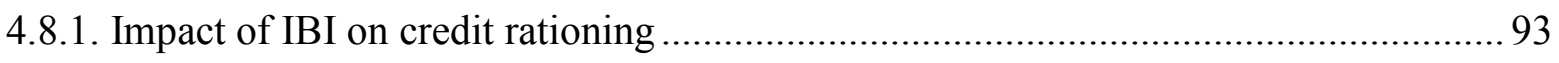

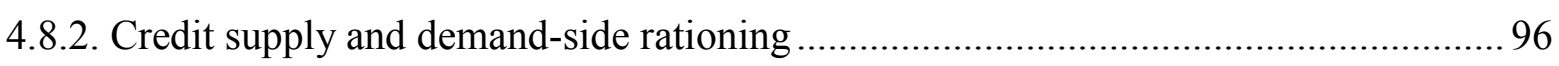

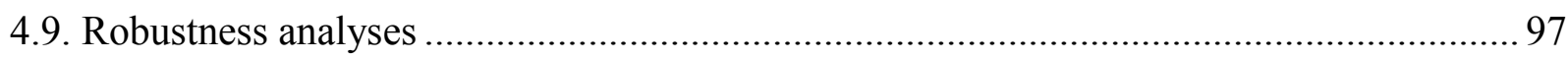

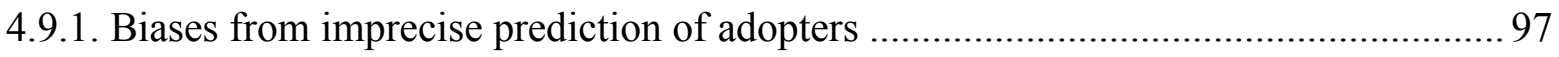




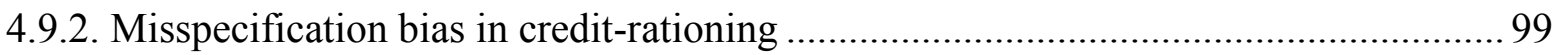

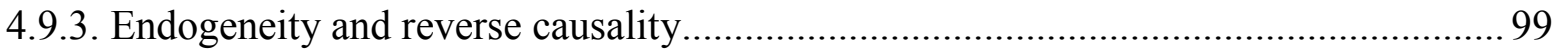

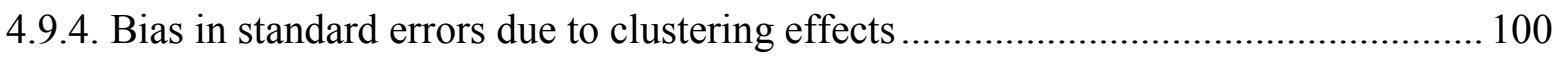

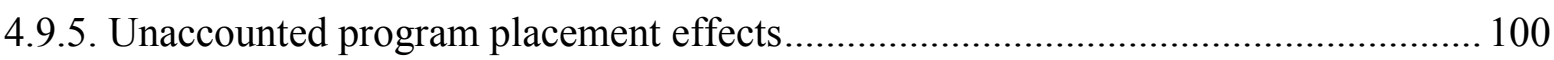

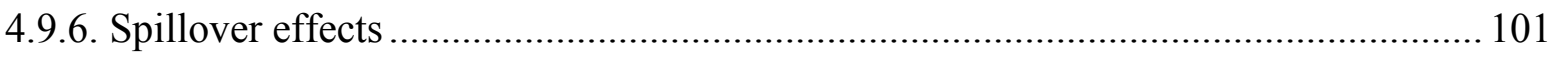

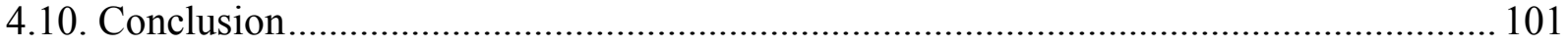

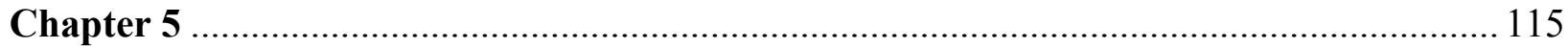

Does index-based insurance improve household welfare? Empirical evidence based on

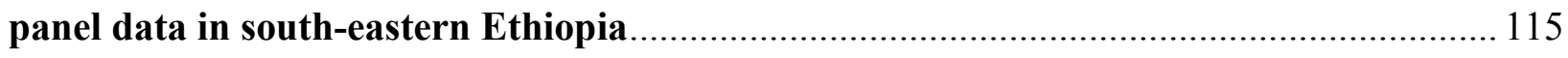

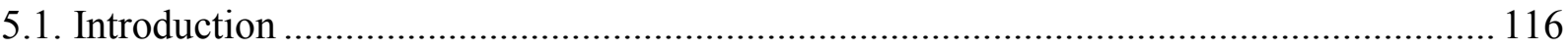

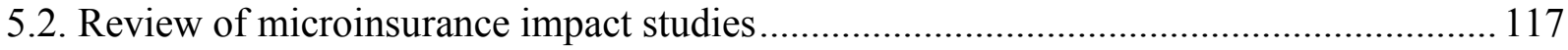

5.3. Study context, IBI intervention, survey design and data............................................. 120

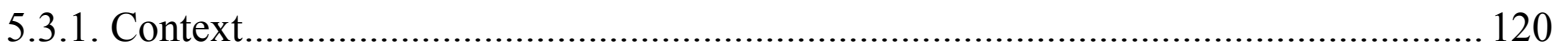

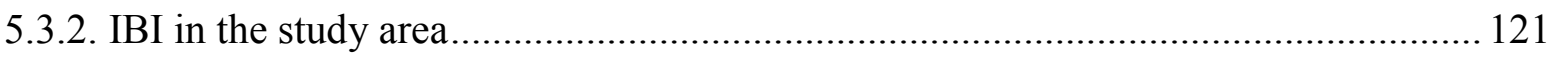

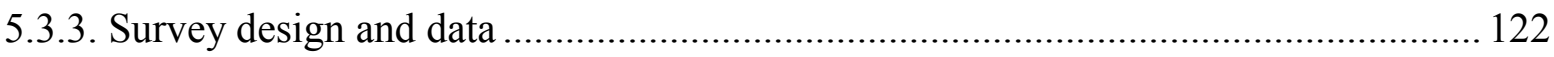

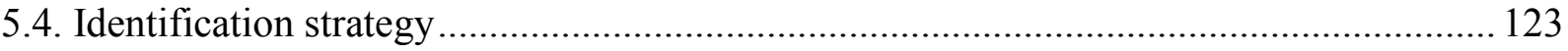

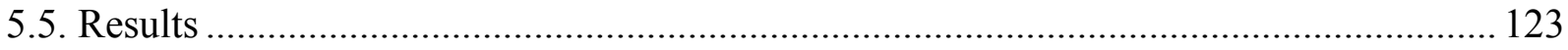

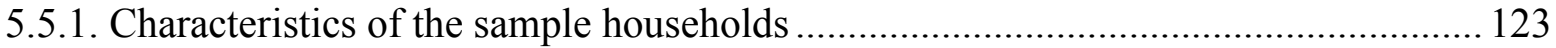

5.5.2. Effects of participation in IBI adoption on household welfare .................................. 125

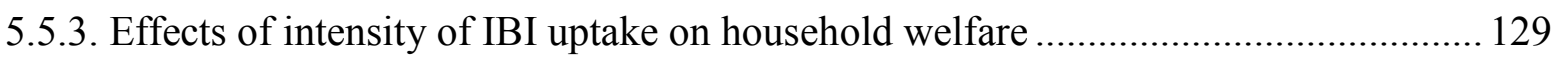

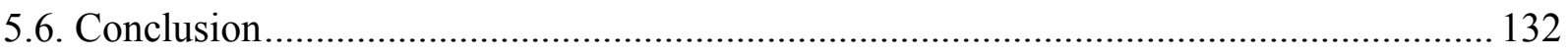

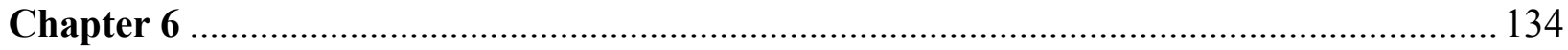

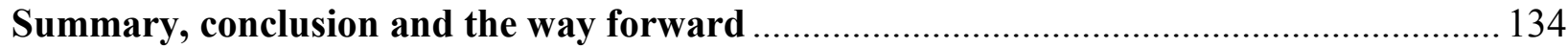

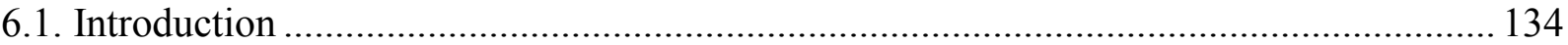

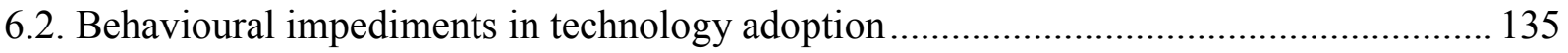

6.3. Relaxing liquidity constraints increases uptake of weather insurance ............................ 137

6.4. Trust holds the key in weather insurance uptake decisions......................................... 138

6.5. Contracts reduce strategic defaults in interlinked insurance-credit intervention ............. 138

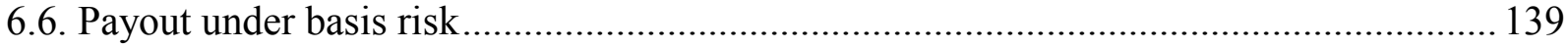

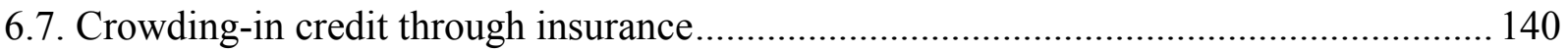

6.8. Impacts of IBI adoption on household welfare: consumption and investment ................ 142

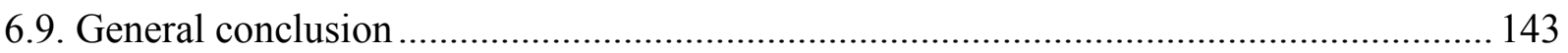

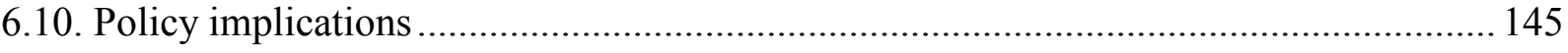

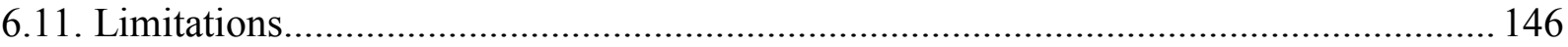




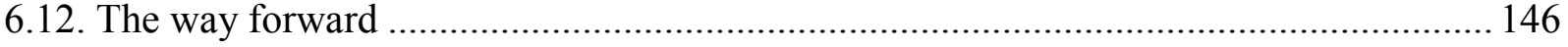

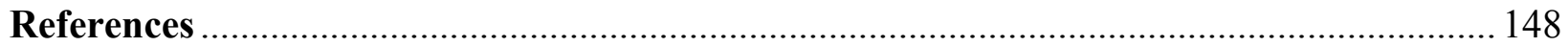

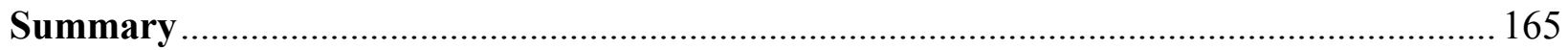

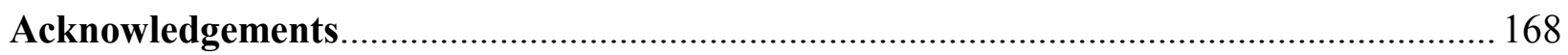




\section{Chapter 1}

\section{Introduction}

\subsection{Shocks and welfare}

Households in developing countries face idiosyncratic and covariate shocks resulting from nature and seasonality-based variations. Exposure to repetitive shock events causes low productivity, slow growth and persistent poverty (Morduch 2004). The majority of smallholders that constitute about two-third of the households in low-income countries rely on agricultural and natural resources for their wellbeing (World Bank 2007; Christiaensen et al. 2011). They practice traditional farming systems that bear little resilience to unprecedented covariate shock events induced by climate change. Weather shocks are evidenced to have a profound impact on the welfare of such poor households (Thomas et al. 2010). The adverse welfare effects of these shocks propagate through the value chain and local linkages. Shocks thus affect both smallholders whose income and consumption directly depend on agriculture, and rural dwellers whose employment and business income indirectly depend on agricultural outcomes. Covariate weather shocks also affect actors in the rural economy (Jayachandran 2006). These include rural producer organizations, workers in agribusiness sector, entrepreneurs, input suppliers and producers of non-tradable goods and services. At macro level, weather shocks undermine the functioning of local, regional and national governments as well as global humanitarian bodies. These agents face stringent budgetary constraints to respond to sudden demand for relief, recovery and rehabilitation during covariate shock events (Cummins et al. 2004).

Shocks occur when risks materialize, resulting in loss of livelihood assets, reduced investment in health, nutrition or education that in turn reinforce vulnerability and perpetuate poverty. Individuals cannot foresee shocks but make subjective probability distributions of their occurrence, failure of which refers to uncertainty. Formal risk management mechanisms like insurance and risk-contingent credit are mostly inaccessible in rural areas. Households heavily rely on a wide range of informal risk-sharing mechanisms including socially-constructed reciprocity obligations, informal social insurance networks and mutual help associations that function on the basis of trust and altruism (Aredo 1993; Coate and Ravallion 1993; Fafchamps and Lund 2003; Aredo 2010). However, these mechanisms are costly, limited in scope and unenforceable. Informal risk-sharing networks that often absorb idiosyncratic shocks offer only 
a limited protection when all community members commonly face covariate weather shocks at a time (Dercon 1996; Barrett 2014). Hence, when covariate shocks strike, since suitable market instruments that manage risks are not in place and informal risk-sharing networks are not effective, rural households respond to shocks by undertaking costly self-insurance measures. Household responses to shocks involve both ex-ante risk management and ex-post shock coping. Evidences reveal that ex-ante risk management strategies as well as ex-post shock coping practices of households entail high welfare costs and, can end in intergenerational poverty.

Ex-ante risk management mechanisms limit the capacity of the poor to surmount a critical poverty threshold. Before shocks occur or ex-ante, since poor households do not have the resources to take preventive measures or absorb shocks, they modify their livelihoods to mitigate risks. They choose low-risk but low-return crops and technologies or sell off productive assets, reduce investments and borrow at high interest rates, all which lead to further indebtedness and poverty. In line with this, Rosenzweig and Binswanger (1993) find that in India since farmers anticipate that uninsured shocks will strike, and that their capacity to cope with shocks is limited, they rationally adjust their behaviour to reduce exposure to shocks and invest in low-risk low-return activities that reduce the expected consequences of shocks. Similarly, in Tanzania, Dercon (1996) finds that poorer farmers grow more sweet potatoes-a low-risk low-return crop - than richer farmers, trading lower risk for a reduction of up to onefourth in average earnings. On the other hand, as risk exposure leaves lenders vulnerable to default by borrowers, uninsured risk is evidenced to commonly limit household's access to credit, especially for the poor who lack collateral to guarantee loan repayment (Besley 1995). Boucher et al. (2008) also identify that during negative shock periods, fear of losing the productive assets to be used as collateral—risk rationing — prohibit smallholder farmers to use a credit line though they own collateralizable assets that lenders are willing to accept to lend them (Caselli 1997). In general, engagement in low-risk but low-return asset and livelihood strategies reduces risks of severe suffering among the poor, but it proportionally limits their growth potential, investment incentives and capacity to adopt technologies. Further, together with the precautionary actions like limits to access credit, such suboptimal production practices can reinforce inherited patterns of chronic poverty (Feder et al. 1985; Eswaran and Kotwal 1990; Morduch 1995; Bardhan et al. 2000; Dercon 2005; Elbers et al. 2007).

Ex-post shock coping mechanisms can also trap poor households into poverty. After shocks or ex-post, rural households persuade shock coping mechanisms like self-insurance through reduced consumption and low expenditure on nutrition that leads to lasting health outcomes like 
stunted child development (Morduch 1995; Islam and Maitra 2012). Furthermore, shocks usually lead household decisions to make pupils withdraw from school for child labour employment on landlord farms, resulting in low human capital development (Beegle et al. 2003; Guarcello et al. 2010). Elabed and Carter (2014) report that farm households pursue shock coping through reducing consumption expenditures to achieve asset smoothing and/or selling productive assets to achieve consumption smoothing. Reducing consumption to protect assets typically has high immediate welfare costs. It can result in irreversible adverse consequences in health (e.g., stunted child development) or in education (e.g., withdrawal of pupils from school with low probability of return), thus perpetuating intergenerational poverty. Selling productive assets like livestock, seeds or land to protect consumption undermines income-generating capacity and can push households into poverty traps from which it will be difficult to escape (Barrett et al. 2007). Other disruptive shock-coping responses include costly labour adjustments through migration and child labour, and costly financial adjustments through dissaving and indebtedness (Gollier 1994). Short-term shocks can thus have unexpected long-term consequences, sometimes lasting across several generations (Premand and Vakis 2010).

Financial market instruments can help rural households to effectively manage idiosyncratic and covariate risks. Poor households in low-income countries have limited access to credit and insurance services. Chantarat (2009) views that if farm households have access to insurance to insure against shocks ex-ante, or access to credit to borrow ex-post, then the adverse welfare effects of risk can be attenuated or eliminated. Hence, risk cannot be the fundamental cause for the prevalence of poverty traps among the poor. Factors that impede the capacity of poor households to surmount the critical poverty threshold are some combinations of financial market imperfections that generate exclusionary mechanisms like insurance and credit rationing. Covariate risks, asymmetric information and high transaction costs are the fundamental causes for the failure of the functioning of formal financial markets in developing countries. Covariate risks in the form of spatially correlated weather-related disasters can cause losses that can exceed the reserves of an insurer or lender. Since insurers and lenders limit their financial service provision due to fear of shocks, potential policyholders or borrowers can be left unprotected. This can explain why crop insurance policies are functional only in countries where governments can take on much of the catastrophic risk exposure faced by insurers (Binswanger and Rosenzweig 1986; Miranda and Glauber 1997; Binswanger-Mkhize 2012). The prevalence of asymmetric information like adverse selection and moral hazard also exposes lenders and insurers to incur losses that exceed the proceeds that they collect from premium and 
interest rates. In addition, transaction costs of financial contracting in rural areas are very high due to limited transportation, communication and legal infrastructures (Renkow et al. 2004; Binswanger and Rosenzweig 1986). High lending costs, combined with the small amount of borrowing by poor households, naturally leads to credit rationing that excludes the poor in equilibrium (Williamson 1986).

Insurance is the obvious policy instrument to manage risks. The form of insurance intervention to be tailored for smallholders, however, depends on the type of shocks. Christiansen and Dercon (2007) report that in rural Ethiopian villages, covariate shocks induced by shortage of rainfall have sharp consumption reducing effects while idiosyncratic shocks have little or no effect on consumption patterns. In northern Mali, Harrower and Hoddinott (2005) document that though occur frequently, idiosyncratic shocks have negligible effects on household consumptions, while covariate shocks were highly correlated with consumption declines. Carter (1997) reveals that in Burkna Faso, smallholder yields are more exposed to covariate risks (54\%) than to idiosyncratic risks (46\%). Udry (1990) similarly found that smallholder farmers in northern Nigeria face more yield exposures to covariate risks (58\%) than to idiosyncratic risks (42\%). These evidences elucidate that households in developing countries are more exposed to covariate shocks than to idiosyncratic ones. Hence, risks that smallholders face are importantly covariate, requiring insurance interventions.

Access to agricultural insurance helps to manage covariate risks that smallholders face. This remains very important particularly when the infrequent but severe character of such shocks make a difficulty to smooth income and consumption through conventional financial products like precautionary saving or credit (Carter et al. 2014). However, though heavily subsidized indemnity-based agricultural insurances have well worked for farm households in developed countries, such services were prohibitive to effectively hedge the covariate risks that smallholders in developing countries face (Glauber 2004; Kovacevic and Pflug 2011). The most important reasons underlying why conventional indemnity-based insurance does not work for smallholder farmers in developing countries are information asymmetry and transaction costs (Hazell 1992; Miranda 1997; Farrin and Miranda 2015). Recent evidences broadly corroborate this fact. Africa, Latin America and Asia account only for 1\%, 2\% and 18\% of the global agricultural insurance premium, respectively (Iturrioz 2009). Hence, over the last decades, indemnity-based crop insurances that constitute the primary formal aggregate risk hedging markets achieved limited success in developing countries due to information asymmetry, high transaction costs and lack of prompt payments (Just et al. 1999). 


\subsection{Index-based insurance}

Index-based insurances (IBIs) are innovations that calculate insurance payouts based on external indices extracted from observable weather or satellite data. In the context of rural financial market development and risk management, IBIs constitute innovative hedging instruments that have an immense potential to mitigate the adverse welfare effects of drought shocks and seasonality-based weather variations, resulting from climate change (Miranda and Vedenov 2001; Barnett et al. 2008; Barrett 2011; Takahashi et al. 2016). The attractive feature of this innovation is that insurance payout is triggered when an objective index of a selective weather variable falls below a given threshold, signalling risk. The index is usually based on a measure of intensity of rainfall, images of vegetative cover on the earth's surface measured by satellite remote sensing, or direct measures of yields for a specific geographic zone that the insurance contract covers (Skees 2008; Zant 2008). Ideally, such index is closely correlated with the insured losses, objectively quantifiable and publicly verifiable in order not to be manipulated by both the insurer and the insured (Barnett et al. 2008; Zant 2008).

IBIs can overcome the classic challenges of rural financial market development at least in three different ways. First, IBIs delink loss assessment from individual behaviour to overcome moral hazard problems. Second, the design of IBIs is based on publicly verifiable data (e.g., rainfall data or satellite-based normalized differential vegetation index (NDVI) data). This partially helps to overcome the problem of adverse selection ${ }^{1}$. However, IBI contracts may still entail spatial adverse selection due to limitations in using low technological resolutions in extracting indices, or intertemporal adverse selection because of extended insurance sales windows. Thirdly, the use of a single index to estimate losses of a group of farmers helps to reduce transaction costs that otherwise would arise from claim verification and contract management. Hence, IBIs uniquely overcome classic incentive problems of information asymmetry and

\footnotetext{
${ }^{1}$ Unlike indemnity-based insurances, farm-level verification is not needed in IBIs. This helps to overcome the problems of moral hazard and adverse selection, as well as the high costs and long delays of claim verifications. In IBIs, instead of monitoring yields or livestock mortality over thousands of dispersed small farms, a prohibitively costly enterprise, measurement is needed only for the level of local rainfall, intensity of vegetation (greenery) measured by NDVI or the average yield per crop production zone. IBI is thus, in principle, a potentially highly cost effective approach to the unresolved issue of first order importance, expectedly to allow delivering much needed insurance to large numbers of smallholder farmers and rural inhabitants in developing countries.
} 
transaction costs associated with insurance claim verification and contract enforcement in the development of rural insurance markets (Barnett et al. 2008). However, the main shortcoming of IBIs is the inherit prevalence of basis risk in the product design.

Basis risk is the imperfect correlation between computed indices and real losses that can jeopardize actual uptake and impact of IBIs (Cummins et al. 2004; Jensen et al. 2014). The main strength in IBI design is its use of a single representative index to assess the losses of a group of individuals. But if the index is weakly correlated with individual farm level losses that it is supposed to insure, IBIs cannot credibly reveal the real value of the loss-payout function (Carter et al. 2014). In developing countries where terrestrial weather stations are sparse, the discrepancy between losses indicated by the index and actual losses realized at farm level is usually high (Clarke et al. 2012). Hence, due to basis risk, IBI provides only a partial (probabilistic) insurance coverage, sometimes with invalid responses. Invalid index signal that constitutes basis risk can arise with two mutually exclusive probabilities in a given insurance period. First, an upward basis risk with false positive probability (FPP) can cause the index to trigger payout though the insured did not incur losses (Elabed et al. 2013). Second, a downside basis risk with false negative probability (FNP) can cause the index not to trigger any or appropriate amount of payout though the insured incurred significant loss. In this way, IBI contracts with high levels of basis risk tend to function like a lottery than insurance (Jensen et al. 2014; Carter et al. 2015).

Downside basis risk has an important implication in enhancing the uptake and economic impacts of IBIs. For instance, in occasions when false negative index is occurred and announced, smallholders have already made out-of-pocket premium payment. They have also incurred losses on their farms but no payout is forthcoming. In this case, the uninsured smallholder remains better off than purchasing the insurance at all. Suppose this happens in a given period, and then potentially, there will be high reservation for uptake of IBIs in the subsequent period. This happens due to economic as well as behavioural reasons. First, cash outflow in the form of out-of-pocket premium payment and the yield loss due to covariate shocks penalizes the wealth of the insured household twice. As a consequence, poor farmers can remain very sensitive to such a double penalty that further ruins their minimum welfare level (Elabed and Carter 2015). Second, the expected but not realized payout can also make the insured to consider the contract as non-trustworthy. Individual buyers worry that if they decide to buy IBI, then their harvest can fail but the payout may not come forth. The state of nature in terms of low or high rainfall is unknown and whether the IBI contract correctly provides payout under low rainfall is 
ambiguous. Hence, due to compound risk and ambiguity aversion behaviour, potential buyers may well avoid the decision to buy IBIs, preferring to stay with their endowment (Elabed and Carter 2015). This decision leaves smallholders uninsured from covariate weather shocks, but the preference is like 'better the devil you know' (Bryan 2010).

\subsubsection{The puzzle of low uptake in IBI}

Carter et al. (2017) state that uptake of IBIs is a battle in progress with success and failure stories. Uptake remains quite low, and many reasons have been put forward to explain this. Preferences (e.g., risk, ambiguity and time preferences) were identified to substantially limit the uptake of IBIs among smallholders. In risk preference analysis, households having diminishing, constant or increasing marginal utility of wealth are categorized as risk-averse, risk-neutral or risk-seeking agents. Suppose these agents were provided with two preferences: a certain option with fixed payoffs, and a gamble with the same expected payoff. Under rational choice, those risk-averse, risk-neutral and risk-seeking households are expected to derive higher, equal and lower utility, respectively, from choosing the certain option with fixed payoff compared with the gamble with the same expected payoff. With non-zero transaction costs ${ }^{2}$, even at actuarially fair premium, taking-up the IBI is not worthwhile for the risk-neutral or risk-seeking household, because such price is above the certainty equivalent of the losses that the household expects to incur. Hence, the demand for IBI is higher for a risk-averse household than risk-neutral or riskseeking households. On top of this, various factors can still limit IBI uptake decision of a riskaverse household. One instance is when the household suspects that due to basis risk, the insurance contract may fail to payout even though losses are incurred. When households incur losses but payouts are not triggered, or vice versa, the IBI product itself becomes a risky product for the household to buy. Other instances that circumscribe the uptake of IBIs for a risk-averse individual arise if the insurer defaults, or if the client distrusts the insurer to make payout. Ambiguity preference also affects IBI uptake decisions because ambiguity-averse households worry that odds depend on their choices, and their choices are always wrong (Dercon 2008; Bryan 2010). From the perspectives of time preference, individual households derive varying discounted future utilities from IBI payouts based on their degree of impatience (Becker and Mulligan 1997; Gneezy and Potters 1997). Households being rational decision makers do

\footnotetext{
${ }^{2}$ Such costs arise from the difficulty of purchasing or renewing the insurance, the opportunity cost of time and the complexity of filing a claim, and the ease with which premiums can be paid and payouts can be collected.
} 
maximize their discounted utility in such a way that they prefer current payoffs over future payoff provided that the utility from the present payoff is greater or equal to the present value of the utility from the future payoffs (Harrison et al. 2007; Harrison et al. 2009; Netzer 2009; Henrich et al. 2010). Therefore, individual discount rates of these households determine their IBI purchase decisions.

Apart from preferences, cognitive factors like understanding insurance actuaries, knowledge about basis risk or trust on IBI product can affect perceptions and value judgements of households about the usefulness of IBIs (Patt et al. 2010). Households' learning from stochastic experience about the occurrence of shocks can also influence their IBI uptake decisions. Recent shock experience or recency bias, information on IBIs that updates with accumulated experience as well as its diffusion through social network groups can influence the decision of households to buy IBI. Barrett (2016) indicated that spatiotemporal adverse selection behaviour of households also limits their IBI uptake. Two forms of adverse selection can prevail in IBI markets. One is intertemporal adverse selection arising from the fact that households often buy less of insurance when they expect good rainfall condition in the future. The other is spatial adverse selection in which households in locations with high covariate risks are more likely to decide to buy IBI. Belissa et al. (2018b) also identified that liquidity constraints play an important role in determining IBI uptake decisions of households. IBI contracts require fixed premium payments during insurance sell windows. Such a time may not necessarily be the harvest time or a time when households have adequate cash inflows. In low-income countries, since saving is limited, formal credit is inaccessible and the informal credit sector charges exorbitant interest rate to lend, rural households can limit their IBI uptake due to their liquidity constraints (Udry 1994: 1995; Ashraf et al. 2006; Matsuyama 2007).

Liquidity in the form of time inconsistency between households' cash inflows and premium payment can thus influence IBI uptake decision of the households. Household specific behavioural and demographic characteristics can also influence IBI purchase decisions. Behavioural determinants including mental accounting, fatalism or status quo bias, (sophisticated) hyperbolic discounting, loss aversion and balanced reciprocity are also evidenced to influence the decision of a household to buy IBIs. Similarly, household characteristics including age, dependency ratio, gender and education can also influence IBI uptake decisions. For instance, the decision of female-headed households to buy or not to buy IBI can be different from those male-headed households. Since women in low-income countries are primarily responsible to manage income shocks, female-headed households can have 
differential responses in adopting insurance technologies. Yet, the way the decision to purchase IBI is made within a household is also an important issue. The interpretation of the gender coefficient in an uptake regression will be radically different in the presence of unitarian households rather than bargaining ones. In the latter case, each household member has a different weight in the decision-making process to buy IBI (Gneezy et al. 2009; Carlsson 2013).

\subsubsection{Impacts of IBI adoption on household welfare}

Economic returns to IBIs is potentially substantial, ranging from inducing households to make more prudential investments, providing better management for consumption risk (through stabilizing savings or asset accumulation) that protect those households from sliding into poverty traps, crowding-in finance for ancillary investment and growth to enhancing local adaptation to climate change (Barnett et al. 2008). Various evidences reveal that where effectively implemented, IBIs have welfare improvement impacts. For instance, recent studies show that adoption of IBIs encourages investment in higher-risk high-return activities with higher expected profits. Focusing on this ex-ante investment risk-taking behaviour, Karlan et al. (2014) found that lack of access to insurance is the limiting factor to investment for maize farmers in Ghana. Using a randomized experiment that allow access to IBI and cash grants, the study revealed that smallholders who purchased IBI increased agricultural investment by $13 \%$. Their study also has important implications for demand, learning and trust in adoption of IBIs ${ }^{3}$. Mobarak and Rosenzweig (2014) used a randomized experiment where IBI is offered to Indian cultivators. Results show that IBI helps cultivators reduce self-insurance and switch to riskier, higher-yield production techniques. They further argue that more risky production in turn destabilizes the labour market and hurts agricultural workers. When the same insurance is offered to farm workers, they respond less to changes in labour demand associated to seasonal weather shocks, helping smooth wages across rainfall states. The study puts the policy implication that weather insurance should be offered to both cultivators and farm workers to avoid the negative spill-over effects of insurance for the first without insurance coverage for the second. Cai et al. (2015) find that insurance for sows significantly increases farmers' tendency

\footnotetext{
${ }^{3}$ Importantly, demand for insurance remains strong even when a full market price is charged, equal to the fair price plus a $50 \%$ premium. At that price, some $50 \%$ of farmers still demand insurance to cover about $60 \%$ of their cultivated area. At the current stage of insurance experimentation, this finding is a rare case where insurance demand holds at market prices. This study also shows that experiencing payouts either oneself or through others is important for demand, indicating the importance of learning and trust (see also Cassar et al. 2017).
} 
to raise sows in south-western China, where sow production is considered as a risky production activity with potentially large returns. In another experiment, Cai (2012) demonstrates that weather insurance induces tobacco farmers in China to increase the land devoted to this risky crop by $20 \%$. This finding implies reduced diversification among tobacco farmers, consistent with less self-insurance. Using an experimental game method, Vargas-Hill and Viceisza (2010) show that insurance induces farmers in rural Ethiopia to take greater, yet profitable risks, by increasing (theoretical) purchase of fertilizer. An important inference from all these evidences is that adoption of IBIs indeed enhances prudential investment risk-taking behaviour among farm households.

IBIs are also evidenced to have impacts on ex-post shock coping. Recent works provide compelling evidences on this. Janzen and Carter (2013) show that access to an index-based livestock insurance (IBLI) in northern Kenya helped pastoral households to smooth their asset and consumption, the two key dimensions of self-insurance. The intervention was undertaken with the realization that pastoral households residing in arid and semi-arid areas were exposed to numerous income shocks that translate into erratic consumption patterns. Income, asset or consumption smoothing is highly necessary as variations result in adverse welfare effects and repeated exposure to downturns reinforces vulnerability (Morduch 1995). The study revealed that after the intervention, poor households are less likely to have to destabilize their consumption in response to drought, while rich households are less likely to have to compromise their accumulated assets. Insured households are observed to be less dependent on food aid and other forms of assistance, which indicates their better ability to cope with shocks. The impact of the intervention was also selective based on wealth position of the households. Fuchs and Wolff (2011) identify the impact of insurance against catastrophic drought to smallholder farmers in Mexico on corn yields, area cultivated in corn, and per capita income and expenditures. They find that insurance coverage induced ex-ante risk management responses with an $8 \%$ increase in corn yields, along with gains in income and expenditures.

Understanding the impacts of IBIs to crowd-in finance for rural investment and growth is another important economic issue. Although little empirical evidence details the interplay of IBI and other financial services like saving and credit, some earlier studies provide insights. In an experimental game conducted in China, Cheng (2014) studies the effect of offering IBI to riskrationed households. More than half of the risk-rationed farmers decide to apply for credit when IBI is available to them. Roughly two-thirds of credit diverters also choose to use their loan for productive investments rather than consumption when IBI is available. The rationale is that IBI 
reduces production risk, but the risk associated with using the loan for consumption remains constant or might even increase, because the household pays for the premium but does not invest. Further insights come from Giné and Yang (2009). They offered maize farmers in Malawi the choice between a loan and a loan plus insurance, which indemnifies them if rainfall is insufficient. Although they expected that farmers would prefer the insured loan, demand for the basic loan was $13 \%$ higher than that for the insured loan. To explain why insurance did not lead to higher demand for credit, these authors suggest that the limited liability clause of the basic loan provided some implicit form of insurance. Carter et al. (2016) argue that a standalone insurance product does not provide additional benefits to farmers who have low collateral. The reason is that, if no formal insurance is available, only farmers with high collateral may choose not to borrow, because they do not want to put their collateral at risk. Cai (2016) also finds that insurance causes households to decrease savings by more than $30 \%$. This implies that households were building up extra precautionary savings in order to better smooth consumption in the case of a shock. Belissa et al. (2018c) examined the impact of IBI on credit rationing in Ethiopia, and found that insurance coverage is positively related to credit use. The study then goes on to examine if the change is associated with reduced demand side or supply-side rationing, finding that the changes are due to differences in supply-side rationing.

Adoptions of IBIs were also evidenced to reinforce the functioning of the extant social insurance mechanisms. In an experiment, Mobarak and Rosenzweig (2012) show that in rural India the existence of informal risk-sharing networks among members of a sub-caste increases demand for IBI when informal risk-sharing covers idiosyncratic losses, reducing basis risk. In this case as well, IBI was evidenced to enable farmers to take on more risk in production. Belissa et al. (2018b) examined whether uptake of IBI can be enhanced by postponing the premium payment towards shortly after harvest, and by marketing the product through the extant social insurance institutions known as Iddirs in Ethiopia. The study found that coaxing the market-based IBI with the predominant social insurance reveals higher uptake than selling such insurance through conventional statutory channels like state-owned cooperative unions. On the other hand, Fuchs and Rodriguez-Chamussy (2011) analysed the impact of insurance payouts on voter behaviour in the 2006 presidential election in Mexico. The unit of analysis is the electoral section, and the question is whether insurance payouts received by farmers in the electoral section in 2005 affected voting behaviour toward the incumbent political party in the 2006 election. The identification strategy is a regression discontinuity based on the threshold rainfall levels that trigger payments of the IBI. They find that disaster relief buys votes. The 
incumbent party is estimated to have gained $8 \%$ more votes where payouts had been made prior to the election (List and Sturm 2006). The conclusion of this review of evidence is thus that, where available and affordable, IBI does work for the intended purposes. It helps to achieve more effective shock coping and less costly risk management. The outcome can be more resilience to climate change as well as less poverty and high growth in the economy.

\subsection{Research objectives}

The overall objective of this dissertation is to investigate innovative mechanisms that enhance the uptake and impact of IBIs on household welfare, in order that IBIs serve as a sustainable risk management tool. Risks are the potential states which individuals cannot control. The search for effective risk management mechanisms is high on the agenda of researchers and policy makers since risks cause significant welfare losses. In the past, indemnity crop insurances that constitute the primary formal insurance markets in developing countries were unsuccessful mainly due to the problems associated with information asymmetry. Recent innovations in IBIs calculate payouts based on satellite (weather) data that delink loss assessment from individual behaviour to overcome moral hazard problems. Hence, these innovations have a potential to manage production and consumption risks. However, basis risk inherently limits the design of IBIs, and micro-level take-up rate is quite low. Moreover, predominant social insurances that manage idiosyncratic risks are often overwhelmed due to covariate shocks or crowd-out effects of formal insurances. This raises concerns how IBI markets can be evaluated and reoriented to have increased impact on household welfare, together with the extant informal social insurances in rural areas. Accordingly, the specific research objectives addressed in this dissertation are thus to: (i) Understand the effects of risk and ambiguity aversion behaviour on IBI uptake decision (ii) Investigate the impacts of improving trust and relaxing liquidity constraints on uptake of IBI (iii) Measure the effects of IBI on promoting households' access to financial markets and (iv) Evaluate the welfare impacts of IBI adoption on household production and consumption behaviour.

\subsection{Methodology}

\subsubsection{Experimental approach}

The use of incentive-compatible experiments for better identification of causal mechanisms has recently attracted the attention of many scholars in development economics research. In testing the efficacy of a given development intervention or the validity of a given economic concept or theory, experiments help researchers to form their own setting (John 2008). Using experimental 
approaches, researchers make random assignment of subjects to treatment and control groups, and form an exogenous variation of the variable(s) of their interest. Field experiments undertaken in socio-economic studies can take one of three different forms. First, many studies employ what is known as artefactual or lab-in-the-field experiment, similar to natural laboratory experiments but with the exception that subjects are drawn from the domain of interest. While the theoretical justifications of this method were elucidated in various studies, relevant empirical applications in developing countries are largely growing. The second category are framed field experiments in which researchers use the context of the experimental setting such as the protocol, information, activities, technology or the product to be used by the participants. Elabed and Carter (2015) used such a framed field experiments to show that compound-risk aversion behaviour of cotton farmers in northern Mali largely affect their willingness to pay for agricultural insurance services. The third category of field experiments often used in social science research are natural field experiments which are undertaken in a natural environment regardless of the recognition of subjects that they are taking part in the experiment.

In the study included in Chapter 2 of this thesis, we used a lab-in-the-field experimental approach to measure the extent to which household behaviour towards risk and ambiguity aversions influence index-based insurance adoption decision. This approach helped us to explicitly understand the subtle contexts of uncertainty, risk and ambiguity aversion, and their respective roles in technology adoption decisions of smallholder farmers in Ethiopia. Similarly, in Chapter 4 and 5, we report the effect of an IBI implemented in a natural field experiment setting on households' credit rationing behaviour. The use of experiment is not however without raising questions associated with ethical considerations. Informed consent among the subjects that take part in the experiment is the first issue to be addressed (Couture 2008). Subjects should provide full consent, without any external coercion and should be able to explicitly realize the consequences of taking part in the experiment. All households who participated in the experiments considered in this study were carefully approached, informed and convinced about the pros and cons of taking part in the experiment. We explained to them and included in our instruments that participants can voluntarily take part in the experiment, and can terminate it at any time that they want. The prominence is on anonymity from the perspectives of both the participants and experimenters to avoid conflict of interest, revenge and retaliation among individual subjects (Gneezy and List 2006; Gerber and Green 2012). 


\subsubsection{Randomized controlled trials (RCT)}

Randomized experiments are designs on which the researcher has full control over the assignment of subjects to the treatment, and such assignment mechanisms do not depend on the observed or unobserved characteristics of the units. This means researchers form their own exogenous variations in the variables of their interest. Since researchers have control over designs, randomization guarantees unbiased allocation of treatments to study participants. In other words, by the virtue that the researcher has full control over the assignment of subjects to the treatment, RCTs uniquely eliminate selection biases in comparing the treatment and control units. Thus, randomized experiments can avoid many of the challenges of observational studies for causal inference. In observational studies the researcher has no control on the assignment of subjects to the treatment. Assignment procedures often depend on a set of observable and unobservable characteristics of the units in the study (Rosenbaum 1995; Imbens and Rubin 2015). Well-designed and implemented RCTs constitute the golden standards in evaluating the treatment efficacy of development interventions (Smith 1976; Wilde 1981; Smith 1982).

However, this does not imply that RCTs provide answers for all causal inference questions. For instance, though randomization is largely seen as the most credible approach that provide a more reliable evidence on causation than observational studies, neoclassical views put that randomizations have no special place in hierarchy of methods to obtain causal inferences (Deaton 2010). The main strengths of RCTs are that they have high internal validity, meaning that RCTs reveal a higher extent to which the causal impacts can be attributed to an intervention. Hence, well-designed RCTs overcome internal validity problems that often plague the reliability of causal inferences from observational studies. However, neither RCTs nor observational studies can effectively overcome the external validity problems. External validity concerns inferences about the extent to which a causal relationship holds over variation in persons, settings, treatments, and outcomes. The main reason is that in experiments involving human subjects, one typically needs informed consent such that individuals typically need to agree to participate in the experiment. But there is nothing that will guarantee that subjects who agree to do so will be similar to those that do not do so (similarity in baseline characteristics), and thus there is nothing that can guarantee that inferences for populations that give informed consent will generalize to populations that do not-external validity (Harrison and List 2004).

In addition, there are various statistical issues with regard to the design and analysis of experimental studies. For instance, in evaluating experiments by comparing sample means for 
the treatment and control group for observably homogeneous and independent subjects, how to make inference about the treatment effect is questionable. On the other hand, when there are observable differences in characteristics among study units, questions revolve around how to account for imbalances between the characteristics of the two groups in order to reveal the randomization has worked. In all experiments, thus, it is important to understand how the results of an experiment can be generalized (Gneezy and List 2006; Banerjee and Duflo 2009; Charness and Genicot 2009; Gerber and Green 2012).

RCTs are major shifts in approach that directly use statistical methods which are justified by the randomization than the conventional sample-based econometrics. The later assumes that treatment assignments are fixed and sample drawing is random. As a result, inference is made based on the argument that subjects are a small random sample of a larger population. In contrast, in RCT, the potential outcome of the subjects in the experiment is fixed, and the assignment of subjects to the treatment is considered as random. However, RCTs do not ensure for a particular trial that the study participants in each treatment group will have similar characteristics (Duflo et al. 2003; Duflo et al. 2007; Wantchekon and Guardado 2011).

But why RCTs are widely viewed as approaches that provide the most credible evidence on causal effects? The usual tests of significance are not precise to compare baseline similarity between treatment groups with control groups. It also can help to identify the covariate(s) that should be included in the model for adjustment. Distribution of baseline attributes simply presented in tabular form is however, rather preferred. For covariate selection, approaches that make use of information on the degree of correlation between the covariate(s) are preferred. Furthermore, it should be noted that stratification and minimisation are not alternatives to covariate adjusted analysis. Of course, these establish the need for covariate adjusted analysis. The common approach in analysis is the intention-to-treat for evaluation of primary outcome measures. Researchers have responsibility to report whether or not the procedure was followed. A major use of results from subgroup analysis is to generate hypothesis for future trials. Though RCTs are golden standards in the comparison of interventions, these remain costly as compared to survey observations.

\subsubsection{Econometric procedures}

We have given due emphasis to the experimental methods in many parts of the studies included in this thesis. For instance, in the RCT part the main focus is on balancing tests than putting a rigorous econometric approach in place. However, this thesis does not limit itself only to 
experimental methods; it also makes use of a variety of econometric models. In Chapter 2 we use discrete choice models to understand the effect of risk and ambiguity aversion not only on a mere uptake, but also on the intensity of IBI adoption among the smallholders. In Chapter 3 the focus of the RCT design was to read the level effects of the multiple treatment arms on uptake. We employed the OLS approach and interpret the constant term as the uptake of the control group. In Chapter 4 the cross-sectional difference-in-difference methods was employed. The procedure was so innovative in various aspects including the combined PSM-DID, and a handwritten program following the Coleman (1999) approach for cross sectional DID. In Chapter 5 we used panel data based DID method to evaluate the impact of IBI adoption on household production and consumption. This approach helps to overcome selection biases in impact evaluations.

\subsection{Relevance of the study}

This research is relevant in terms of its contribution in providing solutions for rural technology adoption when rural livelihoods are confronted with risks. It is also relevant since each chapter of the study provides a novel contribution to the literature in adoption and impact of drought insurance technologies. In Chapter 2, the role of risk and ambiguity aversion in hindering insurance uptake decisions of smallholders in Ethiopia is addressed. This largely contributes to the debates on the relevance of experimental and behavioural economics to understand the role of intrinsic behaviour among households in their technology adoption decisions (Simon 1959; Henrich et al. 2001; Karlan 2005; Henrich et al. 2010). It corroborates evidences by drawing inferences from multiple price elicitation exercises of risk and ambiguity behaviour using labin-the-field experiments. Chapter 3 further discovers innovative mechanisms to overcome the low uptake rate of index-based insurances among smallholders in Ethiopia by improving trust and relaxing liquidity constraints using a randomized controlled trial. This provides compelling evidence on the role of trust in the insurance product or the insurance provider and the role of time inconsistency related liquidity constraints in halting insurance uptake decisions of smallholders. Chapters 2 and 3 together help to identify mechanisms that enhance the demand for index-based insurances. Addressing the role of such important factors as trust, liquidity constraints as well as preferences like risk, ambiguity and time in limiting insurance adoption decisions of smallholders create an opportunity for inclusive risk finance to allow the poor benefit from formal insurances (Banerjee et al. 2011). Chapter 3 also provides evidence on the need for integrating IBIs with indigenous informal insurances that bear trust in order to overcome liquidity and trust problems, and to ensure social insurances to function even under 
exogenous risks. Chapter 4 and 5 measure the impact of index-based insurance adoption on alternative welfare outcomes including households' production, consumption and access to other financial markets like credit. Investigating economic benefits of IBIs in this way is central to the fight against vulnerability and poverty arising from weather-related risks. In general, future climatic scenarios are pessimistic. By 2050, extreme weather events cost $1 \%$ of global annual GDP and 20\% higher in lower income countries (Stern 2007). Hence, developing IBIs help to protect the welfare of vulnerable poor smallholders that dominate the developing world.

\subsection{Outline of the thesis}

In addition to this introductory chapter, this dissertation consists of six chapters. The next chapter examines how risk and ambiguity preferences determine households' insurance uptake decisions. In this chapter we present the role of risk and ambiguity aversion in explaining uptake of weather index insurance. We base this study on a lab-in-the-field experiment combined with a household survey in the Rift Valley Zone of Ethiopia. The third chapter examines whether uptake of index insurance can be enhanced by addressing two issues: liquidity constraints and trust. We assigned a total of 8,579 households grouped in 144 Iddir clusters to various treatment arms in which the type of IBI product, the channel through which the product is sold and the contract that reinforce repayment of a post-ponded premium were varying. We learnt that the innovative IOU insurance product increases uptake by itself, and there is even a high increase in uptake when this product is channelled through Iddirs that households trust with binding social or legal contracts that in turn reinforce repayment. The fourth chapter examines whether index insurance enhances households' access to financial markets. This chapter examined whether and to what extent index insurance reduces credit rationing. Using direct elicitation method (DEM) in line with Boucher et al. (2009) to differentiate between demand and supply-side credit rationing, and a cross-section based difference-in-difference technique, we estimated that adoption of IBI significantly reduces supply-side credit rationing. In Chapter 5, we measure the impact of index insurance on production and consumption behaviour of households. We focus specifically on the question whether and to what extent the use of insurance enhances households' investment in high-risk high-return agricultural inputs, food and non-food consumption. Using an innovative methodology in line with panel data based difference-indifference method, we find that adoption of IBI significantly increases household welfare in terms of both production and consumption behaviour. The final chapter presents the summary of the main results of the thesis. It also discusses some limitations of the thesis, and put forward some issues for further research. 


\title{
Chapter 2
}

\section{Risk and ambiguity aversion behaviour in index-based insurance uptake decision: Experimental evidence from Ethiopia}

\begin{abstract}
Index-based insurance (IBI) is an innovative pro-poor climate risk management strategy that suffers from low uptake. Evidence on the role of behavioural impediments in adoption of IBI is scant. We conducted lab-in-the-field experiments with 1139 smallholders out of whom 596 have adopted IBI in Ethiopia to elicit their risk and ambiguity aversion behaviour, and examine whether risk and/or ambiguity aversion can explain actual IBI uptake decisions. Our study suggests that an increase in risk-aversion increases uptake, but an increase in ambiguityaversion lowers uptake of IBI. We also find evidence that an increase in risk aversion speeds-up the uptake of IBI, while an increase in ambiguity aversion delays the adoption of IBI.
\end{abstract}

Keywords: index-based insurance; risk aversion; ambiguity aversion; lab-in-field experiment

This chapter is based on:

Belissa, Lensink and Asseldonk (2018): Risk and ambiguity aversion behaviour in index-based insurance uptake decision: Experimental evidence from Ethiopia. Under review, Journal of Economic Behaviour and Organization 


\subsection{Introduction}

Production risk is embedded in the day-to-day activities of smallholders. It is mainly driven by nature and seasonality-based variations. Insurance is the obvious risk management option to hedge household welfare from adverse risks. However, indemnity-based crop insurances that constitute the primary formal insurance markets are mostly unsuccessful in developing countries, due to information asymmetries and high transaction costs. Recent innovations in the form of index-based insurances (IBIs) aim to overcome moral hazard and asymmetric information problems by delinking payouts from individual behaviour. Payouts will be made if an objectively determined index falls below a given threshold. The index can be constructed from the intensity of rainfall, images of vegetative cover on the earth's surface, often measured by satellite remote sensing, or area yield losses. Dependable indices of this type should closely correlate with individual yield losses, objectively quantifiable and publicly verifiable in order not to be manipulated by both the insurer and the insured (Barnett et al. 2008; Skees 2008; Zant 2008). An additional advantage is that the use of a single index for a group of farmers in an area for premium rating and determining payouts minimizes transaction and underwriting costs. Thus, IBI is a hedging instrument with immense potential to manage especially drought shocks induced by climate change (Barrett 2011; Takahashi et al. 2016). However, the demand for IBI is sluggish and its uptake remains quite low in developing countries (Carter et al. 2014).

A main reason for the low uptake of IBI is basis risk. Basis risk refers to the imperfect correlation between computed indices and actual losses that can jeopardize actual uptake of IBI (Cummins et al. 2004; Jensen et al. 2014). In the presence of basis risk, a household exposed to production risk who contemplates to purchase IBI faces two hurdles of uncertainty. First, the household anticipates the likelihood of production risk. Second, the individual considers whether the IBI contract validly reflects the losses. While smallholders may be able to anticipate the probable failure of production based on their stochastic experience, they may not be able to comprehend whether the IBI contract accurately triggers payout once losses are incurred. This may imply that risk-averse individuals who are willing to buy IBI to hedge against production risk may withhold their IBI uptake decisions due to the ambiguity surrounding the validity of the insurance contract.

This study examines how risk and ambiguity aversion behaviour of smallholders influence their IBI adoption decisions. Our analysis begins with explaining why risk-exposed smallholders may remain behaviourally reluctant to hedge their production risks with IBI technologies. We argue 
that on top of being risk-averse, most smallholders are ambiguity-averse. As a result, they fear that if they buy any IBI, it may not payout when it is needed (and it may payout when it is not needed). This outcome is in line with the Ellsberg Paradox. Ellsberg (1961) indicated that most individuals prefer events with known probabilities (risk) over events with unknown probabilities (ambiguity). Individuals that exhibit such behaviour are ambiguity-averse (Becker and Brownson 1964). Bryan (2010) further explained that ambiguity-averse individuals 'worry' that odds depend on their choice in such a way that their choices are always wrong.

Previous empirical studies on microinsurance were mostly undertaken within the expected utility (EU) framework. Under EU, a utility maximizing individual buys IBI at an actuarially fair price expecting that it provides a better coverage than remaining uninsured. Particularly, risk-averse households may prefer buying IBI since they derive higher utility from the payouts than the utility from stochastic production. But for a risk-neutral or risk-loving individual, purchasing IBI even at actuarially fair price might not be worthwhile. The reason is that unavoidable transaction costs associated with underwriting, filing a claim and collecting payouts can make the insurance premium to be higher than the certainty equivalent of losses of the buyer (De Bock and Ontiveros 2013). Hence, under EU, the demand for IBI is higher for a risk-averse individual than a risk-neutral or risk-loving one. However, in the presence of basis risk, a riskaverse individual can still have no or limited uptake of IBI due to ambiguity whether the contract accurately reflects the actual losses. The EU framework, thus, under-weighs the adverse effect of ambiguity aversion on IBI uptake, and systematically overstates the demand for IBI (Carter et al. 2015).

There are few earlier studies that have examined the relevance of risk and ambiguity aversion in explaining the low uptake of IBI. Elabed and Carter (2015) conducted a framed field experiment with cotton farmers in Mali to measure the effect of compound-risk and ambiguity aversion on household willingness to pay for microinsurance. Their findings indicate that individuals are willing to pay more for microinsurance contracts with reduced basis risk. Drawing on theoretical justifications, Clarke (2012) indicated that basis risk largely limits the theoretical rational demand for IBIs. However, both studies are based on hypothetical and theoretical cases. Evidence on the effects of ambiguity and risk-aversion on actual uptake of IBI is almost entirely absent. The main contribution of our study is that we assess the effects of risk and ambiguity aversion on actual uptake decisions of smallholders in Ethiopia who have repeated access to IBI. 
The rest of the chapter is organized as follows. Section 2.2 provides a model of IBI adoption under risk and ambiguity, and drives testable hypotheses based on the model. Section 2.3 describes our data sources. We use experimental and survey data collected in 2017, from insured and uninsured smallholders in Ethiopia. Section 2.4 explains the estimation procedures. Section 2.5 presents the main results. First, an increase in risk aversion speeds-up adoption of IBI. Second, ambiguity-averse smallholders have low uptake of IBI. Third, because learning diminishes ambiguity overtime, increased experience in adoption of IBI mitigates the effect of ambiguity aversion on IBI adoption. Section 2.6 concludes the chapter.

\subsection{Risk and ambiguity aversion in IBI adoption decision}

In this section, first we model the inherent basis risk in IBI design as the source of ambiguity, and then formulate IBI adoption as a hierarchical decision through ambiguity and risk aversion. The model gives useful insights on how risk and ambiguity can affect IBI adoption decision. Various previous studies have used parts of this comprehensive model in their theoretical frames (Chantarat et al. 2013; Carter et al. 2017; Elabed and Carter 2015). Consider household $i$ farming at location $l$ in zone $z$ who faces drought risk during season $s$. The yield loss for the zone and the individual household can be specified as: $\omega_{z s}=\bar{\omega}_{z}+\varepsilon_{z s}$ and $\omega_{i l s}=\bar{\omega}_{i l}+\varepsilon_{i l s}$, respectively, where $\omega_{z s}, \bar{\omega}_{z}$ and $\omega_{i l s}, \bar{\omega}_{i l}$ represent the respective current season and long-term average yield loss of the zone and the household. Similarly, $\varepsilon_{z s}$ and $\varepsilon_{i l s}$ represent idiosyncratic components of yield estimates for the zone and the household, respectively. Deviation in yield for the household can be associated with zone level deviation as:

$$
\omega_{i l s}=\bar{\omega}_{i l}+\lambda_{i}\left(\omega_{z s}-\bar{\omega}_{z}\right)+\varepsilon_{i l s}
$$

where $\lambda_{i}$ measures the extent to which yield deviation (loss) of the household closely tracks that of the zone. If $\lambda_{i}=1$, the two losses closely track each other; but if $\lambda_{i}=0$, the two losses are statistically independent of each other. It also holds that $0 \leq \lambda_{i} \leq 1$ and the expected value of $\lambda_{i}$ overall the location is necessarily 1 . Since measuring yield deviation at individual farm level is not cost-effective, a representative index at zone level is estimated as follows.

$$
\omega_{z s}-\bar{\omega}_{z}=f\left(S_{z s}\right)+\xi_{z s}
$$

where $f\left(S_{z s}\right)$ is a yield loss predictor transformational function whose signal maps the index of a weather variable into average zone-level yield outcomes, and $\xi_{z s}$ is a prediction error. 
Substituting eq. (2.2) into eq. (2.1), and solving for $\omega_{i l s}$ provides household-level yield loss in terms of the index signal $f\left(S_{z s}\right)$ as follows.

$$
\omega_{i l s}=\bar{\omega}_{i l}+\lambda_{i} f\left(S_{z s}\right)+\lambda_{i} \xi_{z s}+\varepsilon_{i l s}
$$

where $\xi_{z s}$ and $\varepsilon_{i l s}$ represent design risk and idiosyncratic risk, respectively. These are the two main elements of basis risk. In practice, technocrats that design IBI reduce eq. (2.3) into a simplified underlying index and estimate yield loss of the individual household as:

$$
\omega_{i l s}-\bar{\omega}_{i l}=f\left(S_{z s}\right)
$$

Though simple, eq. (2.4) is consistent only under three restricted conditions: perfect correlation between yield deviations at the zone and individual household level $\left(\lambda_{i}=1\right)$; no design risk $\left(\xi_{z s}=0\right)$ and no idiosyncratic risk $\left(\varepsilon_{i l s}=0\right)$. From this, we can understand the inherent prevalence of basis risk in IBI design. First, imperfect correlation can potentially exist between yield deviations at zone and individual farm levels (i.e., $\lambda_{i} \neq 1$ ). Second, technocrats can commit design risk (i.e., $\xi_{z s} \neq 0$ ). Third, idiosyncratic yield losses cannot be zero (i.e., $\varepsilon_{i l s} \neq 0$ ). Hence, under one or more of these conditions, a non-zero basis risk can prevail in IBI contract design. As a result, IBI contract can trigger invalid index. Then, payout for insured farmers will be inaccurate since it is determined based on the estimated index of yield loss. This forms the primary source of ambiguity for smallholders to buy IBI. Suppose the rate of estimated index of yield loss is $\omega_{e}$, the actual rate of loss realized by a household is $\omega_{r}$ and the rate of exit (minimum level of index for payout) is $\omega_{t}$, then, the rate of payout, $\omega_{p}$, is determined as:

$$
\omega_{p}=\left\{\begin{aligned}
\omega_{r}-\omega_{t} ; & \omega_{t} \leq \omega_{r} \leq \omega_{e} \\
0 ; & \text { otherwise }
\end{aligned}\right.
$$

The rate of payout determined, $\omega_{p}$, is then used to calculate the monetary payouts. Suppose $q_{z s}$ and $p_{z s}$ represent the number of IBI policies and the premium per policy, respectively, then, value insured per policy is $p_{z s} q_{z s}$. The premium of the value insured per policy, $p r m_{z s}$, is determined as:

$$
\operatorname{prm}_{z s}\left(\omega_{r} / \omega_{t}, q_{z s}, p_{z s}\right)=E\left[\operatorname{Max}\left(\omega_{r}-\omega_{t}, 0\right) p_{z s} q_{z s}\right]
$$

where $E$ is the expectation operator taken over the distribution of the index. Based on this, the actuarially fair premium rate quoted as the percentage of total value of yield insured per policy is $\operatorname{prm}_{z s}\left(\omega_{r} / \omega_{t}, q_{z s}, p_{z s}\right)=E\left[\max \left(\omega_{r}-\omega_{t}, 0\right)\right]$. The insurer adds up all sum insured and determines total expected payout, $p m t_{z s}$, at the end of each season $s$ as: 


$$
p m t_{z s}\left(\omega_{r} / \omega_{t}, q_{z s}, p_{z s}\right)=\sum \operatorname{Max}\left(\omega_{r}-\omega_{t}, 0\right) p_{z s} q_{z s}
$$

Note that $\omega_{t}$ is estimated from $f\left(S_{z S}\right)$. Its precision depends on the level of technology to capture weather variables. Accuracy in measurement of rainfall or the degree of resolution of vegetative images determines the quality of the index. In developing countries, since weather infrastructure is poorly developed, IBI designs may reveal invalid indices leading to inaccurate computation of losses and payouts. Hence, households can be ambiguous about the benefit from such contracts.

Let $q$ and $1-q$ represent the probabilities with unknown distribution that IBI contract triggers valid and invalid index, respectively. Production risk that smallholders face due to drought depends on whether the state of rainfall is wet or dry. Let $p$ and $1-p$ represent the probabilities of wet and dry weather, respectively. Assume further that the smallholder pays premium $y$ to buy IBI, out of the current wealth $x$, in expectation of future payout $z$, conditional on facing losses amounting to $l$. The net random wealth of the smallholder is one of the four outcomes: $p q(y-x)$ under wet weather and valid index; $p(1-q)(y-x+z)$ under wet weather and invalid index; $q(1-p)(y-x-l+z)$ under dry weather and valid index or $(1-p)(1-$ $q)(y-x-l)$ under dry weather and invalid index.

EU theory assumes that individuals make their final decision by reducing the joint outcomes into a single payoff. This is known as the Reduction of Compound Lottery (RCOL) axiom (Segal 1990). But empirical studies show that due to illiteracy, emotions and other behavioural factors, computing and reducing lengthy joint probabilities is practically undoable and cumbersome (Carlin 1992; Budescu and Fischer 2001). Decision makers facing joint lotteries act surprisingly averse to such lotteries, effectively behaving as if the final simplified probabilities are unknown or ambiguous (Elabed and Carter 2015). Hence, their behaviour is inconsistent with EU maximizing behaviour (Borghans et al. 2009; Bryan 2010). An alternative theory of preference that can better handle such scenario is the subjective expected utility (SEU). SEU assumes that individuals make subjective cost-benefit analysis in making decisions that involve multiple conditions.

In this chapter, we study the behavior of smallholders to buy IBI from the SEU perspective. Specifically, we used the KMM model to characterize the double-hurdle uncertainty that they face. KMM assumes that individuals order their risk and ambiguity preferences based on their SEU. Some recent studies also employed this model (Elabed and Carter 2015; Barham et al. 2014; Izhakian and Benninga 2011; Neilson 2010). In KMM, risk and ambiguity preferences are 
represented by two utility functions in a double expectation form. Risk preference is captured by the curvature of the utility of wealth and ambiguity preference is explained by a second-stage utility function defined over the expected utility of wealth. Accordingly, we denote risk and ambiguity preferences of smallholders by $U(x)$ and $V(x)$, respectively. Each smallholder makes the decision to buy IBI, $d$, in a set of competing decisions, $D$, (i.e., $d \in \mathrm{D}$ ). Household decision that maximizes the final wealth from buying IBI is thus:

$$
x(d) \equiv\left\{E_{q} V\left[E_{p} U(x)\right]: d \epsilon D\right\}
$$

where $x(d)$ is wealth; $E$ is expectation operator; $U$ and $V$ are risk and ambiguity preferences. Subscripts, $p$ and $q$ are probabilities of wet weather and valid index. Eq. (2.8) shows the joint probability density function of the final wealth in the pattern that households order their risk and ambiguity preferences over wealth. Both $U$ and $V$ are assumed to be strictly increasing, twice differentiable von-Neumann Morgenstern type of utility functions with $U^{\prime}, V^{\prime}>0$, and $U^{\prime \prime}, V^{\prime \prime} \leq$ 0 . Using KMM allows us to separately understand risk and ambiguity preferences in experimental set ups. With KMM model, the objective function of a household that maximizes the net final wealth from buying IBI, under the four alternative joint probability outcomes can be expressed as follows:

$$
x(d) \equiv\left\{q V E_{p}\left[U\left(x / \omega_{t}=\text { valid }\right)\right]+(1-q) V E_{p}\left(x / \omega_{t}=\text { invalid }\right): d \epsilon D\right\}
$$

where $\omega_{t}=$ valid and $\omega_{t}=$ invalid, represent valid and invalid index, respectively. When the index signal is invalid $(q \neq 1)$, as it may happen in most cases, the final wealth outcome of a household from making the decision to buy IBI, $d$, is:

$x(d) \equiv\{q\langle V p U(y-x)+(1-p) U(y-x-l+z)+(1-q) V p U(y-x)+1-p U(y-x-l)\rangle: d \epsilon \mathrm{D}\}$

However, if the index signal is valid $(q=1)$, then, eq. (2.10) reduces to a simple contract in which only risk (but not ambiguity) matters. This can be represented as follows:

$$
x(d) \equiv\{p U(y-x)+(1-p) U(y-x-l+z): d \epsilon \mathrm{D}\}
$$

Smallholders base IBI adoption decisions on their subjective expected utility (SEU). They make the decision to adopt IBI $d$ through a subjective cost-benefit analysis. Let $x(d)$ represent the benefits from adopting IBI and $c_{r}(d)$ and $c_{a}(d)$ represent the costs associated with risk and ambiguity in making decision $d$, respectively. Since IBI uptake is binary, decision $d$ is a discrete 
choice with $d=1$, for households who decided to buy IBI and $d=0$ for those who decided not to buy. Optimal choice of the household is maximized as:

$$
\text { Choose } d^{*}=\left\{\begin{array}{l}
1 \\
0
\end{array}\right\} \text { when } x(1)-c_{r}(1)-c_{a}(1)\left\{\begin{array}{l}
\geq \\
<
\end{array}\right\} x(0)-c_{r}(0)-c_{a}(0)
$$

Eq. (2.12) shows that the decision to adopt IBI $(d=1)$ is preferred when the benefit from this decision $x(1)$ is higher, and when the costs of risk aversion $c_{r}(1)$ and ambiguity aversion $c_{a}(1)$ are lower. Eq. (2.12) is consistent with some empirical works. Highly profitable technologies entail higher adoption rates (Foster and Rosenzweig 2010). But new technologies can met low demand if smallholders perceive that these are risky (Feder et al. 1985; Foster and Rosenzweig 2010). Bryan (2010) shows that when there is imprecise knowledge, ambiguity reduces the adoption of insurance technologies. Barham et al. (2014) rather indicate that risk aversion has no effect while ambiguity aversion has a large positive effect on adoption of agricultural technologies. This unusual finding was argued to be due to the context of the study that US farmers can have a different risk and ambiguity attitude from smallholders in developing countries.

We derive three hypotheses based on the model in eq. (2.12). First, higher risk aversion levels increase uptake of IBIs. This is the case when the cost of risk under adoption is lower than the cost of risk if adoption of IBI is not undertaken. This means $c_{r}(1)<c_{r}(0)$ in eq. (2.12). Riskaverse households can expect that the benefit (payout) from buying IBI can well-compensate the losses due to production risks. The cost of risk aversion associated with adoption is also expected to be lower than the cost associated with not to adopt. Second, increase in ambiguity aversion decreases uptake of IBI. Eq. (2.12) reflects this case when $c_{a}(1)>c_{a}(0)$. Ambiguityaverse households worry that if they decide to buy IBI, then their harvest can fail and the payout may not come forth. If this happens, out-of-pocket premium payment and the yield loss sums up to a higher cost, penalizing their wealth twice. Ambiguity-averse households thus can become reluctant to take-up IBI during the early adoption phase. Third, the effect of ambiguity aversion on IBI adoption diminishes overtime. Due to learning, experience in use of IBI and diffusion of information about IBI, smallholders can have a better understanding about IBI technology. This can help to reduce the effect of ambiguity on adoption decisions. The level of basis risk can also be minimized through improved IBI contract design overtime (Carter et al. 2014). Hence, the effect of ambiguity aversion on IBI adoption would likely be minimal overtime. 


\subsection{Data sources}

\subsubsection{Study area}

We conducted this study in the Rift Valley zone of Ethiopia. The area is a semi-arid plain plateau with a low-land agro-ecology. It receives a very low level of annual average rainfall. The pattern and intensity of rainfall exhibits considerable spatial and temporal variation, with a bimodal type of distribution. Rainfall seasons are from May to August and during October and November. Moisture stress and drought frequently causes devastating crop failure, rampant livestock mortality and herd collapse (Biazin and Sterk 2013). Major droughts in the area include the 2015-16 drought which followed the historical trend of droughts during 1973-74, 1983-84, 1991-92, 1999-2000, 2005-06 and 2011-12 (Dercon 2004). Households in the area are smallholder subsistence farmers who mainly produce maize and wheat. They often face drought-induced income shocks that translate into erratic consumption patterns. Households employ various ex-post shock coping mechanisms including less meals per day, distress livestock sells, a reduction in investments in farm inputs like fertilizer and improved seed varieties, withdrawal of pupils from school for casual labour, renting land and family labour for local landlords and wage employment on floriculture farms held by foreign investors. Future drought shock predictions in Ethiopia are pessimistic, and the wide crop-livestock mixed farming system in arid and semi-arid areas like the Rift Valley zone were projected to transform into extensive systems to respond to the risks of climate change (Hulme et al. 2001; Meinke and Stone 2005; Thornton et al. 2010). Hence, innovative drought risk management mechanisms that transfer risks like IBI can be useful for smallholders in this area to complement on-farm (ex-ante and ex-post) adaptation strategies.

\subsubsection{IBI adoption in the study area}

Oromia Insurance Company (OIC) and Japan International Cooperation Agency (JICA) jointly launched IBI for crops in the study area in 2013, to improve the resilience of households in the face of climate change. The scheme was initially implemented in five districts: Boset, Bora, Ilfata, Adamitullu-Jido-Kombolcha and Arsi Negele. Major targeted stable food crops to be insured include maize, wheat, barley and teff. The IBI product sold in the study area is a vegetation index crop insurance (VICI). The product is marketed and sold twice per year in April and September, months preceding the two rainy seasons, to provide coverage against losses during the seedling and flowering stages of crop growth. VICI is designed based on the intensity of vegetation cover or greenery on the earth's surface. Greenery level is measured by a 
satellite indicator known as normalized differential vegetation index (NDVI). VICI design is based on NDVI data of 16 years, extracted at a geospatial resolution of $1 \mathrm{~km} \times 1 \mathrm{~km}$ from the GeoNetCast System. NDVI reflects the already accumulated result of rain on crop growth. It is a primary measurement with no assumptions or calibrations. It is the proven standard index, in use by all early warning units globally. Actual decal NDVI data for a given period is calculated for a set of households grouped in similar agro-ecological zones known as crop production system (CPS) zones. In pricing the product, it is assumed that since uptake gradually increases, it is possible to pool more risks across areas with greater geo-spatial variations that can help to reduce transaction costs and to reduce the loading factor. OIC estimates nearly about one out of six households who purchased IBI may face losses, and hence, the premium amounts $15 \%$ of the sum insured. During each sales window, a household that decides to buy IBI pays a premium of ETB 100 (USD 27.5) per policy. Payout calculation procedures are based on every decal (10 days) NDVI data generated. In computing payouts, OIC uses a linearly proportional indemnification (LPI) approach. For instance, for a single insurance with premium of ETB 100, the maximum payout is 100/0.15 which is about ETB 667. Using the LPI approach, for instance, in areas where the index indicates a 50\% loss, a partial payout of about ETB 333.5 is paid to the farmers. Institutional, technological and contractual innovations were also made to reduce basis risk. Initially, OIC attempted a twin-tracking system about the IBI index. Based on this, payout on the basis of the satellite index ranges between $12 \%-68 \%$ while payout as per the field assessment ranges between $10 \%-70 \%$. This indicates that there is some degree of correlation between the losses indicated by the index and the actual losses revealed at the ground.

\subsubsection{Sampling and survey procedures}

We used a multi-stage random sampling technique with probability proportional to size to identify our final units of observation. More concretely, we first selected three districts (Bora, Adamitullu-Jido-Kombolcha and Arsi Negele) out of the five districts covered by the IBI project. Next, we identified a random sample of kebeles $^{4}$ covered by IBI in these three districts. Thirdly, we referred the roaster of each kebele consisting of list of smallholders. In addition, from OIC, we also collected the list of adopters, and the years in which each household was engaged in adoption over the period 2013-2017. Based on the list of adopter and non-adopter households, we randomly selected a total of 1139 households for the survey. From these, 596 households were adopters of IBI while the remaining 543 were non-adopters. We conducted a household survey in 2017. The survey was administered in each kebele about one month prior to the

\footnotetext{
${ }^{4} \mathrm{~A}$ kebele is the smallest administrative unit, next to district in Ethiopia.
} 
execution of the experiments. We collected data on a wide range of topics including household and village characteristics as well as IBI adoption history. There are two IBI adoption cycles per year that provide coverage for the seedling and flowering stages of crop growth, respectively. We categorized households who adopted IBI during the first 3 adoption cycles as early adopters, and those who joined the adoption process during the last 3 cycles as late adopters. Similarly, we considered households who dropped the adoption for at least 3 cycles as dropouts, and those who did not dropout for more than 3 cycles as persistent adopters. Accordingly, from the total adopters, 187 households were late adopters, 260 households were dropouts and 149 households were persistent adopters.

\subsubsection{Experiments}

We conducted incentivized lab-in-the-field experiments to separately elicit risk and ambiguity aversion attitudes of the smallholders. All households who participated in the survey were invited for the experiment. We used multiple price list (MPL) protocols to elicit risk and ambiguity aversion (Andersen et al. 2006). The experiment sessions were undertaken at the Farmers' Training Centre (FTC) in each kebele. In each session, respondents were introduced with the purpose of the experiments. We explained the opportunities for respondents to keep their winnings from the experimental games and to receive payments for show-up. Enumerators explained at the outset that payoffs for the experiments were part of a research grant from a project, and that individuals running the experiment received no personal gains from the experiments or the payoffs made to participants. Such explanation was meant to minimize the extent to which participants might assume that the experimenters would benefit if respondents earned less. The ambiguity experiment involves choosing from a risky option with 50-50 probability, and an ambiguous option with unknown probability. Our procedures are similar to the procedures followed in previous studies (Holt and Laury 2002; Harrison and Rutström 2009; Ross et al. 2010; Barham et al. 2014). The risk experiment involves choosing from a safe option (100\% sure) and a risky option with $50-50$ probability. With slight modification, this design is similar to the protocols used in many studies (Binswanger 1980; Holt and Laury 2002; Brick et al. 2012). All respondents started with playing the ambiguity game before the risk game to avoid anchoring effects ${ }^{5}$. The ambiguity game consists of 11 decisions (see the details in Appendix

\footnotetext{
${ }^{5}$ As the probabilities for the risk game are much more explicitly known than the probabilities in the ambiguity game, if respondents are allowed to play the risk game before the ambiguity game, they may fix their choices to a given row in the risk game and then choose the same row in the ambiguity game.
} 
1A). There were two bags to play the ambiguity game: bag 1 for the risky option and bag 2 for the ambiguous option. Each bag contains 10 pens, some of which were blue and some of which were red. The win or loss in the ambiguity game depends on whether the respondent draws a blue or red pen. For each of the 11 decisions, drawing a blue pen resulted in a gain of ETB 20, while drawing a red pen awards nothing. Respondents had to make their decisions without any prior information about the number of blue and red pens in the ambiguity bag. Respondents were confronted with varying enumerators. Moreover, the proportion of blue and red pens in Bag 2 differed for each participant. After all respondents made the 11 decisions, they were asked to draw one card from another bag containing 11 cards, serially numbered 1 to 11 , in order to determine the payout for the ambiguity game. The payoff was determined as follows. For instance, consider a respondent who draws card No. 7 in the ambiguity game. Then, we refer the colour of the pen that this respondent has drawn in decision 7. If $\mathrm{s}(\mathrm{he})$ has drawn the blue pen, we pay the respondent Birr 20. But if $s($ he) has chosen the red pen, we pay him/her nothing.

Second, respondents played the risk game. The risk game also consists of 11 decisions. Each decision was a choice between two lotteries: Lottery A with a sure payoff and lottery B, a risky payoff with 50-50 probability. The risky option of the risk game was played by flipping a coin by the respondents (see Appendix 1B). Similar to the ambiguity game, after respondents made the 11 decisions, they were asked to draw one card from a bag containing 11 cards, serially numbered 1 to 11 . The card number drawn was considered for the final payment of the respondent for the risk game. That means, corresponding to the card number that each respondent draws, we see their choice. If the respondent has chosen the safe option, we pay the respondent the amount of the safe option. But if the respondent has opted for the risky option, we let the respondent toss a coin, and consider her/his payment based on the flip of the coin.

The average payoff per participant including the ETB 15 payment for show-up was about ETB 38.5. This payment was well above the opportunity cost for labour in the area. For example, one full day's agricultural wage like daily wage from works on floriculture farm (a known daily work in the area) was approximately ETB 50. All the 1139 households have taken part in the experiments. A total of 13 enumerators and one coordinator have worked for about 30 days to conduct the experiments. Enumerators informed the participants that discussing with each other about the choices in the experiment is not allowed. Each participant is asked to carefully think over and decide their choices. The two experiments together have approximately taken about $2 \frac{1}{2}$ hours per respondent. Our experiments were scheduled after typical morning farm activities. 


\subsection{Estimation procedures}

\subsubsection{Measuring risk and ambiguity aversion parameters}

Constant relative risk aversion (CRRA) and constant relative ambiguity aversion (CRAA) values were determined based on individual responses in the experiments. The outcome of the experiments in terms of the measures of CRRA and CRAA values are presented in Tables 2.1A and $2.1 \mathrm{~B}$, respectively. We determined the CRRA values following the procedures in previous studies (Barham et al. 2014; Ross et al. 2010). Accordingly, we assume that household risk preferences entirely exhibit a constant relative risk aversion with a utility function over payoff given by $U(x)=x^{1-\eta} /(1-\eta)$, where $U(x)$ is the expected utility of payoffs under the safe option, $x$ is the payoff under the risky option, and $\eta$ is the CRRA (Pratt 1964). Thus, the CRRA ranges presented under column 6 in Table 2.1A were determined using this function. Then, the CRRA for each respondent is determined based on the row in the risk experiment at which a respondent switches for the first time from the safe option to the risky option. Specifically, we set the CRRA values for each respondent at the minimum value of the range since this reflects the minimum risk aversion level of the respondent. For instance, in Table 2.1A, consider a respondent who chooses the safe option in the first 7 decisions, and switched-off to the risky option on the $8^{\text {th }}$ decision. Since the minimum value of the CRRA range determined using the above function corresponding to the $8^{\text {th }}$ decision is 0.68 , we assigned the value $\eta=0.68$ for all respondents in this category. Our data shows that the proportion of respondents falling in this category is $16 \%$. Similar procedures were undertaken to determine the CRRA values for each respondent. Table $2.1 \mathrm{~A}$ shows that cumulatively about $53 \%$ of the respondents were risk-averse or risk-neutral with $\eta \geq 0$.

Similarly, in determining the CRAA values for each respondent, we assume that abiguity preferences of the households exhibit a constant relative ambiguity aversion with a utility function over payoff given by $V(x)=1-e^{-\tau x} / 1-e^{-\tau}$, for $\tau>0$ and $V(x)=x$ for $\tau=0$, whereas $V(x)$ represents the expected utility of payoffs under the risky option and $\tau$ estimates the coefficient of ambiguity aversion (Klibanoff et al. 2005). As we have 11 decisions involving choices between risky and ambiguous options in the experiment (see Table 2.1B), we consider the switch-off points to determine the minimum CRAA value for each respondent (Barham et al. 2014; Ross et al. 2010). The switch-off point is the row at which the respondent prefers the ambiguous option over the risky option for the first time. 
Table 2.1A: Risk aversion $(\boldsymbol{\eta})$

\begin{tabular}{|c|c|c|c|c|c|c|c|c|}
\hline (1) & $(2)$ & (3) & (4) & (5) & $(6)$ & (7) & $(8)$ & $(9)$ \\
\hline Task & Safe option & Risky option & $\mathrm{EV}^{\text {safe }}$ & $\mathrm{EV}^{\text {risky }}$ & CRRA ranges & $\eta$ & $\%$ & Risk aversion class* \\
\hline 1 & ETB 18 & 0.5 of ETB $20 ; 0.5$ of ETB 0 & ETB 18 & ETB 10 & $-1.73<\eta<-1.49$ & -1.73 & 0.01 & Extremely risk-loving \\
\hline 2 & ETB 16 & 0.5 of ETB $20 ; 0.5$ of ETB 0 & ETB 16 & ETB 10 & $-1.49<\eta<-0.95$ & -1.49 & 0.04 & Highly risk-loving \\
\hline 3 & ETB 14 & 0.5 of ETB $20 ; 0.5$ of ETB 0 & ETB 14 & ETB 10 & $-0.95<\eta<-0.42$ & -0.95 & 0.15 & Risk-loving \\
\hline 4 & ETB 12 & 0.5 of ЕТВ $20 ; 0.5$ of ЕTВ 0 & ETB 12 & ETB 10 & $-0.42<\eta<0.00$ & -0.42 & 0.16 & Moderately risk-loving \\
\hline 5 & ETB 10 & 0.5 of ЕТВ $20 ; 0.5$ of ЕТВ 0 & ETB 10 & ETB 10 & $0.00<\eta<0.11$ & 0.00 & 0.12 & Risk-neutral \\
\hline 6 & ETB 8 & 0.5 of ETB $20 ; 0.5$ of ETB 0 & ETB 8 & ETB 10 & $0.11<\eta<0.41$ & 0.11 & 0.10 & Slightly risk-averse \\
\hline 7 & ETB 6 & 0.5 of ETB $20 ; 0.5$ of ETB 0 & ETB 6 & ETB 10 & $0.41<\eta<0.68$ & 0.41 & 0.09 & Moderately risk-averse \\
\hline 8 & ETB 4 & 0.5 of ЕТВ $20 ; 0.5$ of ЕТВ 0 & ETB 4 & ETB 10 & $0.68<\eta<0.97$ & 0.68 & 0.08 & Risk-averse \\
\hline 9 & ETB 2 & 0.5 of ЕTВ $20 ; 0.5$ of ЕТВ 0 & ETB 2 & ETB 10 & $0.97<\eta<1.37$ & 0.97 & 0.15 & Highly risk-averse \\
\hline 10 & ETB 1 & 0.5 of ETB $20 ; 0.5$ of ETB 0 & ETB 1 & ETB 10 & $1.37<\eta<3.76$ & 1.37 & 0.07 & Very high risk-averse \\
\hline 11 & ETB 0 & 0.5 of ETB $20 ; 0.5$ of ETB 0 & ETB 0 & ETB 10 & $3.76<\eta<\infty$ & 3.76 & 0.03 & Extremely risk-averse \\
\hline
\end{tabular}

*Note: The classifications in Table 2.1A are based on the terminologies used in Holt and Laury (2002: p 1649). The CRRA values at each switch off points were estimated entirely assuming the CRRA utility function $U(x)=x^{1-\eta} /(1-\eta)$, for $\eta \neq 1$, and $U(x)=\log x$ for $\eta=1$. For instance, for a respondent that switches at the $5^{\text {th }}$ row, we compute the CRRA by equalizing the expected utility of the risky lottery with the expected utility of the safe lottery (i.e., $\left.E U_{R}^{5}=E U_{S}^{5}\right)$, where $E U_{R}^{5}=$ $[(0.5 * 20)+(0.5 * 0)=10]$, and $E U_{S}^{4}=E(10)=10$. Then, substituting, $U(x)=10$, and $x=10$, and solving for the equation, $U(x)=x^{1-\eta} /(1-\eta)$, provides, $\eta=0$

Table 2.1B: Ambiguity aversion $(\tau)$

\begin{tabular}{|c|c|c|c|c|c|c|c|c|}
\hline (1) & (2) & (3) & (4) & (5) & (6) & (7) & (8) & (9) \\
\hline Task & Risky option & Ambiguous option & EV $^{\text {risky }}$ & $\mathrm{EV}^{\text {ambiguous }}$ & CRAA ranges & $\tau$ & $\%$ & Ambiguity aversion class* \\
\hline 1 & 1.0 of ETB $20 ; 0.0$ of ETB 0 & $?(20), ?(0)$ & ETB 20 & {$[?(20)]$} & $-\infty<\tau<-2.57$ & -3.50 & 0.00 & Extremely ambiguity-loving \\
\hline 2 & 0.9 of ETB $20 ; 0.1$ of ETB 0 & $?(20), ?(0)$ & ETB 18 & {$[?(20)]$} & $-2.57<\tau<-1.93$ & -2.57 & 0.03 & Highly ambiguity-loving \\
\hline 3 & 0.8 of ETB $20 ; 0.2$ of ETB 0 & $?(20), ?(0)$ & ETB 16 & {$[?(20)]$} & $-1.93<\tau<-0.31$ & -1.93 & 0.22 & Ambiguity-loving \\
\hline 4 & 0.7 of ETB $20 ; 0.3$ of ETB 0 & $?(20), ?(0)$ & ETB 14 & {$[?(20)]$} & $-0.31<\tau<0.00$ & -0.31 & 0.18 & Moderately ambiguity-loving \\
\hline 5 & 0.6 of ETB $20 ; 0.4$ of ETB 0 & $?(20), ?(0)$ & ETB 12 & {$[?(20)]$} & $0.00<\tau<0.07$ & 0.00 & 0.15 & Ambiguity-neutral \\
\hline 6 & 0.5 of ETB $20 ; 0.5$ of ETB 0 & $?(20), ?(0)$ & ETB 10 & {$[?(20)]$} & $0.07<\tau<0.18$ & 0.07 & 0.05 & Slightly ambiguity-averse \\
\hline 7 & 0.4 of ETB $20 ; 0.6$ of ETB 0 & $?(20), ?(0)$ & ETB 8 & {$[?(20)]$} & $0.18<\tau<0.27$ & 0.18 & 0.04 & Moderately ambiguity-averse \\
\hline 8 & 0.3 of ETB $20 ; 0.7$ of ETB 0 & $?(20), ?(0)$ & ETB 6 & {$[?(20)]$} & $0.27<\tau<0.69$ & 0.27 & 0.07 & Ambiguity-averse \\
\hline 9 & 0.2 of ETB 20; 0.8 of ETB 0 & $?(20), ?(0)$ & ETB 4 & {$[?(20)]$} & $0.69<\tau<1.17$ & 0.69 & 0.16 & Highly ambiguity-averse \\
\hline 10 & 0.1 of ETB $20 ; 0.9$ of ETB 0 & $?(20), ?(0)$ & ETB 2 & {$[?(20)]$} & $1.17<\tau<2.53$ & 1.17 & 0.09 & Very high ambiguity-averse \\
\hline 11 & 0.0 of ETB $20 ; 1.0$ of ETB 0 & $?(20), ?(0)$ & ETB 0 & {$[?(20)]$} & $2.53<\tau<\infty$ & 2.53 & 0.01 & Extremely ambiguity-averse \\
\hline
\end{tabular}

*Note: For the ambiguous option, with uninformative prior, the probability distribution of the payoff is unknown. This is indicated with the question in pair as ? (20), and ?(0) under column 3 and with [?(20)] under column 5 in Table 2.1B. In each row, we calculate the expected value of the risky option. Though we cannot calculate the expected value of the ambiguous option, we can, however, construct ranges for the possible CRAA values as given in column 6 . The CRAA values at each switch off points were estimated entirely assuming the CRRA utility function $V(x)=1-e^{-\tau x} / 1-e^{-\tau}$, for $\tau>0$ and $V(x)=x$ for $\tau=0$. Based on the minimum value of the CRAA ranges, we can determine the $\tau$ values corresponding to each row as given under Column (7) of Table 2.1B. 
For instance, consider a respondent who persistently chooses the risky option for the first 3 decisions, and switch-off to the ambiguous option at the $4^{\text {th }}$ decision row in Table $2.1 \mathrm{~B}$. The CRAA for this respondent is $\tau=-0.31$, and the respondent is considered as ambiguity-loving. Table 2.1B presents the minimum CRAA $(\tau)$ and the percentage of the respondents in this category is $18 \%$. While there is no definitive way to estimate the minimum coefficient of relative ambiguity aversion for those who always chose the ambiguous option (since the minimum could be negative infinity), this behaviour remains rational. It simply implies extreme ambiguity lovingness. Thus, we assign these smallholders with CRAA of -3.50 .

Table 2.1B shows the proportion of respondents in each ambiguty aversion class. It shows that in sum, about $57 \%$ of the respondents were ambiguity-averse or ambiguity-neutral with $\tau \geq 0$. The risk and ambiguity aversion values determined in this study are consistent with the estimates in various studies in developing countries. The share of ambiguity-averse individuals ranges between 42 to 61 percent (Akay et al. 2012). In our sample, 57\% of the respondents are ambiguity-averse or ambiguity-neutral while the remaining $43 \%$ were ambiguity-loving. Camerer and Weber (1992) report that $50 \%$ of their sample were ambiguity-averse.

\subsubsection{Summarizing the data}

Table 2.2 presents summary statistics separately for the entire sample, categories of adopters (late adopters, dropouts and persistent adopters) as well as non-adopters. Column 1 of Table 2.2 shows that, the mean CRAA for the entire sample is -0.29 . The mean CRAA values are -0.35 and -0.22 for adopters and non-adopters, respectively.

Similarly, the corresponding mean CRAA values are $-0.24,-0.40$ and -0.41 for late adopters, dropouts and persistent adopters, respectively. Column 5 in Table 2.2 presents the p-values of the t-test for the difference-in-means between adopters and non-adopters of IBI. The two groups have statistically significant differences in their ambiguity aversion behaviour. Adopters are less ambiguity-averse than non-adopters. But in terms of their risk behaviour, adopters are more risk-averse than non-adopters. Table 2.2 also shows that the average coefficient of relative risk aversion is 0.16 for the entire sample; while the corresponding CRRA values are 0.23 and 0.08 for the sample of adopters and non-adopters, respectively. 
Table 2.2: Summary statistics of IBI adopter and non-adopter households

\begin{tabular}{|c|c|c|c|c|c|c|c|}
\hline \multirow[t]{2}{*}{$\underline{\text { Variables }}$} & \multirow[t]{2}{*}{$\begin{array}{c}\text { (1) } \\
\text { Full sample } \\
\end{array}$} & & $\begin{array}{c}(2) \\
\text { Adopters } \\
\end{array}$ & & \multirow[t]{2}{*}{$\begin{array}{c}(3) \\
\text { Non-adopters } \\
\end{array}$} & \multirow[t]{2}{*}{$\begin{array}{c}(4) \\
\text { Mean difference } \\
\end{array}$} & \multirow[t]{2}{*}{$\begin{array}{c}\text { (5) } \\
\text { T-value } \\
\end{array}$} \\
\hline & & Late & Dropout & Persistent & & & \\
\hline Ambiguity aversion & $\begin{array}{l}-0.29 \\
(1.11)\end{array}$ & $\begin{array}{l}-0.24 \\
(1.07)\end{array}$ & $\begin{array}{l}-0.40 \\
(1.13)\end{array}$ & $\begin{array}{l}-0.41 \\
(1.16)\end{array}$ & $\begin{array}{l}-0.22 \\
(1.09)\end{array}$ & $\begin{array}{c}0.13 \\
(0.07)\end{array}$ & $2.04 * *$ \\
\hline Risk aversion & $\begin{array}{c}0.16 \\
(1.01)\end{array}$ & $\begin{array}{c}0.41 \\
(1.04)\end{array}$ & $\begin{array}{c}0.07 \\
(1.01)\end{array}$ & $\begin{array}{c}0.27 \\
(1.07)\end{array}$ & $\begin{array}{c}0.08 \\
(0.98)\end{array}$ & $\begin{array}{l}-0.15 \\
(0.06)\end{array}$ & $-2.52 * *$ \\
\hline Time preference & $\begin{array}{c}0.26 \\
(0.21)\end{array}$ & $\begin{array}{c}0.25 \\
(0.19)\end{array}$ & $\begin{array}{c}0.28 \\
(0.25)\end{array}$ & $\begin{array}{c}0.20 \\
(0.18)\end{array}$ & $\begin{array}{c}0.26 \\
(0.21)\end{array}$ & $\begin{array}{c}0.01 \\
(0.01)\end{array}$ & 1.05 \\
\hline Age & $\begin{array}{c}40.56 \\
(12.36)\end{array}$ & $\begin{array}{c}41.61 \\
(11.41)\end{array}$ & $\begin{array}{c}41.36 \\
(11.76)\end{array}$ & $\begin{array}{c}40.56 \\
(10.25)\end{array}$ & $\begin{array}{c}39.81 \\
(13.42)\end{array}$ & $\begin{array}{l}-1.43 \\
(0.73)\end{array}$ & -1.96 \\
\hline Gender & $\begin{array}{c}0.84 \\
(0.37)\end{array}$ & $\begin{array}{c}0.89 \\
(0.32)\end{array}$ & $\begin{array}{c}0.83 \\
(0.37)\end{array}$ & $\begin{array}{c}0.86 \\
(0.35)\end{array}$ & $\begin{array}{c}0.82 \\
(0.38)\end{array}$ & $\begin{array}{l}-0.03 \\
(0.02)\end{array}$ & -1.58 \\
\hline Education & $\begin{array}{c}2.39 \\
(1.15)\end{array}$ & $\begin{array}{c}2.51 \\
(1.07)\end{array}$ & $\begin{array}{c}2.45 \\
(1.15)\end{array}$ & $\begin{array}{c}2.62 \\
(1.15)\end{array}$ & $\begin{array}{c}2.25 \\
(1.16)\end{array}$ & $\begin{array}{l}-0.26 \\
(0.07)\end{array}$ & $-3.80 * * *$ \\
\hline Family size & $\begin{array}{c}7.00 \\
(2.80)\end{array}$ & $\begin{array}{c}7.98 \\
(3.25)\end{array}$ & $\begin{array}{c}7.22 \\
(2.55)\end{array}$ & $\begin{array}{c}7.46 \\
(3.06)\end{array}$ & $\begin{array}{c}6.43 \\
(2.54)\end{array}$ & $\begin{array}{l}-1.09 \\
(0.16)\end{array}$ & $-6.67 * * *$ \\
\hline Dependency ratio & $\begin{array}{c}0.50 \\
(0.20)\end{array}$ & $\begin{array}{c}0.52 \\
(0.19)\end{array}$ & $\begin{array}{c}0.49 \\
(0.21)\end{array}$ & $\begin{array}{c}0.48 \\
(0.19)\end{array}$ & $\begin{array}{c}0.50 \\
(0.21)\end{array}$ & $\begin{array}{c}0.00 \\
(0.01)\end{array}$ & 0.14 \\
\hline Land size in qarxi & $\begin{array}{c}8.83 \\
(8.65)\end{array}$ & $\begin{array}{c}9.93 \\
(10.52)\end{array}$ & $\begin{array}{c}9.64 \\
(8.53)\end{array}$ & $\begin{array}{c}9.91 \\
(13.04)\end{array}$ & $\begin{array}{c}7.78 \\
(5.99)\end{array}$ & $\begin{array}{l}-2.02 \\
(0.51)\end{array}$ & -3.96 \\
\hline Livestock size (TLU) & $\begin{array}{c}9.61 \\
(8.00)\end{array}$ & $\begin{array}{c}10.31 \\
(8.27)\end{array}$ & $\begin{array}{l}10.52 \\
(8.24)\end{array}$ & $\begin{array}{c}10.91 \\
(10.52)\end{array}$ & $\begin{array}{c}8.57 \\
(6.79)\end{array}$ & $\begin{array}{l}-1.98 \\
(0.47)\end{array}$ & -4.19 \\
\hline Distance from market & $\begin{array}{c}1.72 \\
(1.03)\end{array}$ & $\begin{array}{c}1.63 \\
(1.07)\end{array}$ & $\begin{array}{c}1.87 \\
(1.12)\end{array}$ & $\begin{array}{c}1.87 \\
(1.10)\end{array}$ & $\begin{array}{c}1.64 \\
(0.95)\end{array}$ & $\begin{array}{l}-0.15 \\
(0.06)\end{array}$ & $-2.52 * *$ \\
\hline Extension contact & $\begin{array}{c}0.94 \\
(0.24)\end{array}$ & $\begin{array}{c}0.93 \\
(0.26)\end{array}$ & $\begin{array}{c}0.95 \\
(0.21)\end{array}$ & $\begin{array}{c}0.99 \\
(0.08)\end{array}$ & $\begin{array}{c}0.92 \\
(0.27)\end{array}$ & $\begin{array}{l}-0.04 \\
(0.01)\end{array}$ & $-2.50 * *$ \\
\hline Crop production zone (CPZ) & $\begin{array}{c}0.71 \\
(0.45)\end{array}$ & $\begin{array}{c}0.51 \\
(0.50)\end{array}$ & $\begin{array}{c}0.81 \\
(0.39)\end{array}$ & $\begin{array}{c}1.00 \\
(0.00)\end{array}$ & $\begin{array}{c}0.65 \\
(0.48)\end{array}$ & $\begin{array}{l}-0.12 \\
(0.03)\end{array}$ & $-4.37 * * *$ \\
\hline Observations & 1139 & 187 & 260 & 149 & 543 & 1139 & \\
\hline
\end{tabular}

$* * * \mathrm{p}<0.01, * * \mathrm{p}<0.05, * \mathrm{p}<0.1$. Standard deviations are in parentheses. T-values compare differences between adopters and non-adopters.

$$
{ }^{\mathrm{a}} S D_{\text {differenceinmeans }}=\sqrt{\frac{S D_{1}^{2}}{N_{1}}+\frac{S D_{2}^{2}}{N_{2}}}
$$


Similarly, the average coefficients of relative risk aversion among late adopters, dropouts and persistent adopters are $0.41,0.07$ and 0.27 , respectively. The estimated coefficients of risk aversion parameter in this study are relatively lower than the value indicated in previous studies. This may be attributed to the experimental design ${ }^{6}$. Compared to non-adopters, IBIadopters are more educated and have larger family size. Moreover, IBI-adopters are more distant from markets and make more frequent contact with extension agents compared with non-adopter households. Adopters and non-adopters also differ on the basis of their local climate conditions.

\subsubsection{Empirical strategy}

We estimate the effects of risk and ambiguity aversion on IBI adoption decision of the households as follows:

$$
d_{i}^{*}=\alpha+\theta(\eta)_{i}+\delta(\tau)_{i}+\beta Z_{i}+\varepsilon_{i ;} \quad d_{i}=1, \text { if } d_{i}^{*}>0
$$

where $d_{i}^{*}$ is an unobserved latent variable; $d_{i}$ is adoption decision of individual household $i$; $\eta$ and $\tau$ are the respective CRRA and CRAA values (directly measured from the experiments); $Z_{i}$ is a vector of covariates affecting IBI adoption including household specific demographic characteristics like age, sex, education and dependency ratio; factors influencing IBI purchase decisions like time preference, trust in the insurer, marketing (promotion), peer influence and intertemporal adverse selection; household resources like land and livestock size, household services like access to market and extension services as well as crop production zone; and $\varepsilon_{i}$ is the error term.

\footnotetext{
${ }^{6}$ Literature identifies two main causes for this: the order effect and the comparative ignorance. Fox and Tversky (1995) found some evidence of ambiguity aversion using a within subjects experimental design. But when the study subjects evaluate one prospect in isolation using a between subjects design, the evidence of ambiguity aversion was not revealed. These authors argue that such effects are due to the comparative ignorance. Fox and Weber (2002) further argue that due to order effects, if a participant makes two decisions, the first decision will be analysed non-comparatively whereas the second will be analysed comparatively. Accordingly, measures of ambiguity aversion are lower in experiments such as ours where the ambiguous bet comes before the risky bet. This suggests that we may underestimate ambiguity and risk-aversion.
} 
We use eq. (2.13) to estimate the effects of risk and ambiguity aversion on IBI adoption in three different ways. First, decision $d_{i}^{*}$ is a discrete choice with $d=1$ for adopters and $d=0$ for non-adopters. Thus, we estimate the effects of risk and ambiguity aversion on the probability of IBI adoption decision of the households using a binary logit. Second, limiting $d_{i}^{*}$ to a mere binary adoption choice imposes the restriction that ambiguity or risk aversion has the same effect on every level of adoption. But we expect that the effect of risk or ambiguity aversion can vary with the intensity or frequency of adoption. Hence, to examine the effect of risk and ambiguity aversion on intensity of IBI adoption, we estimate OLS model where the dependent variable is the number of months that the smallholders have been adopting IBI over the period 2013-2017. Third, a common characteristic of smallholders in technology adoption is that after a group of farmers start adoption in a given year, some of them may continue while others tend to dropout. In IBI adoption, some households who adopted during the initial phase continued while others dropped out. Accordingly, the effects of risk and ambiguity aversion on IBI adoption can vary overtime. For instance, due to learning, the effect of ambiguity aversion on insurance adoption can decrease overtime (Bryan 2010). To understand this, we run separate binary logit regressions measuring the effects of risk and ambiguity aversion on late, dropout and persistent adoption decisions of the households.

\subsection{Results}

\subsubsection{Impacts of risk and ambiguity aversion on the probability of IBI adoption}

Table 2.3 suggests that an increase in risk aversion increases uptake of IBI while an increase in ambiguity aversion reduces the uptake of IBI. Both risk aversion and ambiguity aversion significantly affect adoption of IBI in the model without controls, as well as the specifications with a full set of controls.

Note, however, that risk aversion and ambiguity aversion are borderline significant if both variables are included simultaneously in the specification with the full set of controls (see column 11 and 12). Our findings suggest that risk-averse smallholders try to insure against production risks by adopting IBI. Yet, due to basis risk, ambiguity-averse smallholders are reluctant to buy IBI. 
Table 2.3: Effects of risk and ambiguity aversion on probability of IBI adoption

\begin{tabular}{|c|c|c|c|c|c|c|c|c|c|c|c|c|}
\hline & (1) & (2) & (3) & (4) & $(5)$ & (6) & (7) & $(8)$ & (9) & $(10)$ & $(11)$ & (12) \\
\hline Variables & Coefficients & $\begin{array}{c}\text { Marginal } \\
\text { effects }\end{array}$ & Coefficients & $\begin{array}{c}\text { Marginal } \\
\text { effects }\end{array}$ & Coefficients & $\begin{array}{c}\text { Marginal } \\
\text { effects }\end{array}$ & Coefficients & $\begin{array}{c}\text { Marginal } \\
\text { effects }\end{array}$ & Coefficients & $\begin{array}{c}\text { Marginal } \\
\text { effects }\end{array}$ & Coefficients & $\begin{array}{c}\text { Marginal } \\
\text { effects }\end{array}$ \\
\hline Risk aversion & $0.149 * *$ & $0.037 * *$ & & & $0.122 *$ & $0.030^{*}$ & $0.156 * *$ & $0.039 * *$ & & & 0.116 & 0.029 \\
\hline & $(0.060)$ & $(0.015)$ & & & $(0.064)$ & $(0.016)$ & $(0.073)$ & $(0.018)$ & & & $(0.078)$ & $(0.019)$ \\
\hline Ambiguity aversion & & & $-0.110^{* *}$ & $-0.027 * *$ & -0.070 & -0.017 & & & $-0.140^{* *}$ & $-0.035^{* *}$ & -0.103 & -0.026 \\
\hline Age & & & $(0.054)$ & & $(0.058)$ & $(0.014)$ & & & $(0.066)$ & $(0.017)$ & $(0.070)$ & $(0.018)$ \\
\hline & & & & & & & $0.018 * * *$ & $0.004 * *$ & $0.017 * * *$ & $0.004 * *$ & $0.018^{* * *}$ & $0.004 * *$ \\
\hline Sex & & & & & & & $\begin{array}{l}(0.007) \\
-0.098\end{array}$ & -0.024 & -0.080 & $\begin{array}{l}0.002) \\
-0.020\end{array}$ & $\begin{array}{l}(0.007) \\
-0.099\end{array}$ & $\begin{array}{l}(0.002) \\
-0.025\end{array}$ \\
\hline & & & & & & & $(0.217)$ & $(0.054)$ & $(0.217)$ & $0.054)$ & $(0.218)$ & $(0.054)$ \\
\hline Education & & & & & & & $0.182 * *$ & $0.045^{* *}$ & $0.176^{* *}$ & $0.044 * *$ & $0.180 * *$ & $0.045^{* *}$ \\
\hline & & & & & & & $(0.076)$ & $(0.019)$ & $(0.076)$ & $0.019)$ & $(0.076)$ & $(0.019)$ \\
\hline Dependency ratio & & & & & & & -0.184 & -0.046 & -0.180 & -0.045 & -0.195 & -0.049 \\
\hline & & & & & & & $(0.364)$ & $(0.091)$ & $(0.364)$ & $0.091)$ & $(0.365)$ & $(0.091)$ \\
\hline Time preference & & & & & & & -0.534 & -0.133 & $-0.569^{*}$ & $-0.142 *$ & $-0.599^{*}$ & $-0.150 *$ \\
\hline & & & & & & & $(0.333)$ & $(0.083)$ & $(0.336)$ & $0.084)$ & $(0.337)$ & $(0.084)$ \\
\hline Trust in the insurer & & & & & & & $1.582 * * *$ & $0.361 * * *$ & $1.629 * * *$ & $0.370 * * *$ & $1.612 * * *$ & $0.367 * * *$ \\
\hline & & & & & & & $(0.248)$ & $(0.046)$ & $(0.249)$ & $0.046)$ & $(0.250)$ & $(0.046)$ \\
\hline IBI promotion & & & & & & & $1.218 * * *$ & $0.292 * * *$ & $1.209 * * *$ & $0.290 * * *$ & $1.210 * * *$ & $0.290 * * *$ \\
\hline & & & & & & & $(0.191)$ & $(0.042)$ & $(0.192)$ & 0.042 ) & $(0.192)$ & $(0.042)$ \\
\hline Peer influence & & & & & & & $0.758 * * *$ & $0.186 * * *$ & $0.726 * * *$ & $0.179 * * *$ & $0.740 * * *$ & $0.182 * * *$ \\
\hline & & & & & & & $(0.168)$ & $(0.040)$ & $(0.168)$ & $0.040)$ & $(0.168)$ & $(0.040)$ \\
\hline IAS & & & & & & & $0.386^{* * *}$ & $0.096 * *$ & $0.380 * * *$ & $0.095 * *$ & $0.383 * * *$ & $0.096^{* *}$ \\
\hline & & & & & & & $(0.144)$ & $(0.036)$ & $(0.144)$ & $0.036)$ & $(0.144)$ & $(0.036)$ \\
\hline Land size in qarxi & & & & & & & $0.027 * *$ & $0.007 * *$ & $0.027 * *$ & $0.007 * *$ & $0.027 * *$ & $0.007 * *$ \\
\hline & & & & & & & $(0.011)$ & $(0.003)$ & $(0.011)$ & $0.003)$ & $(0.011)$ & $(0.003)$ \\
\hline Livestock size (TLU) & & & & & & & $0.034 * * *$ & $0.009 * * *$ & $0.035 * * *$ & $0.009 * * *$ & $0.035 * * *$ & $0.009 * * *$ \\
\hline & & & & & & & $(0.011)$ & $(0.003)$ & $(0.011)$ & $0.003)$ & $(0.011)$ & $(0.003)$ \\
\hline Distance from market & & & & & & & 0.087 & 0.022 & 0.079 & 0.020 & 0.083 & 0.021 \\
\hline & & & & & & & $(0.076)$ & $(0.019)$ & $(0.076)$ & $0.019)$ & $(0.076)$ & $(0.019)$ \\
\hline Extension contact & & & & & & & 0.472 & 0.116 & 0.481 & 0.118 & 0.471 & 0.116 \\
\hline & & & & & & & $(0.307)$ & $(0.073)$ & $(0.306)$ & $0.073)$ & $(0.306)$ & $(0.073)$ \\
\hline CPS zone & & & & & & & -0.014 & -0.003 & -0.014 & -0.004 & -0.014 & -0.003 \\
\hline & & & & & & & $(0.014)$ & $(0.004)$ & $(0.014)$ & $0.004)$ & $(0.014)$ & $(0.004)$ \\
\hline District 1 & & & & & & & -0.418 & -0.104 & -0.430 & -0.106 & -0.420 & -0.104 \\
\hline & & & & & & & $(0.276)$ & $(0.067)$ & $(0.276)$ & $0.067)$ & $(0.277)$ & $(0.068)$ \\
\hline District 2 & & & & & & & -0.321 & -0.080 & -0.298 & -0.074 & -0.314 & -0.078 \\
\hline & & & & & & & $(0.223)$ & $(0.055)$ & $(0.223)$ & $0.055)$ & $(0.224)$ & $(0.055)$ \\
\hline Constant & 0.071 & & 0.062 & & 0.055 & & $-4.385^{* * *}$ & & $-4.398 * * *$ & & $-4.375^{* * * *}$ & \\
\hline & $(0.060)$ & & $(0.061)$ & & $(0.062)$ & & $(0.670)$ & & $(0.671)$ & & $(0.671)$ & \\
\hline Observations & 1,139 & 1,139 & 1,139 & 1,139 & 1,139 & 1,139 & 1,139 & 1,139 & 1,139 & 1,139 & 1,139 & 1,139 \\
\hline
\end{tabular}

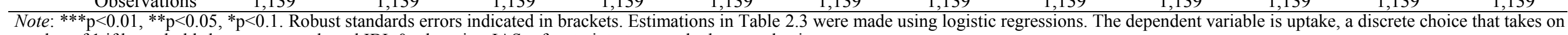
a value of 1 if households have ever purchased IBI; 0 otherwise. IAS refers to intertemporal adverse selection. 


\subsubsection{Impacts of risk and ambiguity aversion on intensity of IBI adoption}

Table 2.4 presents the effects of risk and ambiguity aversion on intensity of IBI adoption. It indicates that an increase in risk aversion increases the number of months that households stay in IBI adoption while an increase in ambiguity aversion decreases the number of months that households stay in IBI adoption phase, with and without including all covariates.

The marginal effects with no other covariates indicate that a unit increase in the experimental risk measure (as households become more risk-averse), increases households' stay in adoption by 0.963 months. Table 2.4 also shows that ambiguity aversion significantly decreases the adoption of IBI. The marginal effects indicate that an increase in the experimental ambiguity measure (as smallholders become more ambiguity-averse) decreases the households' stay in IBI adoption by 1.298 months ( $p$-value $<0.001$ ), controlling for all covariates (See column 5 ).

Risk aversion, however, is not significant if it is included with ambiguity aversion and with all set of covariates (See column 6). Likely, in making their IBI purchase decisions, smallholders might have given due weight for the ambiguity surrounding the IBI contract, but they may give relatively less attention for the stochastic occurrence of production risk. Similar to this study, Barham et al. (2014) finds that risk aversion has only a small impact on the timing of adoption of agricultural technologies, while ambiguity aversion has a large impact on adoption. Ross et al. (2010) also finds that risk aversion does not impact farmers' adoption of rice varieties in Mali while ambiguity aversion does. 
Table 2.4: Effects of risk and ambiguity aversion on intensity of IBI adoption

\begin{tabular}{|c|c|c|c|c|c|c|}
\hline Variables & (1) & (2) & (3) & (4) & (5) & (6) \\
\hline Risk aversion & $\begin{array}{l}0.963^{*} \\
(0.572)\end{array}$ & & $\begin{array}{c}0.445 \\
(0.612)\end{array}$ & $\begin{array}{c}0.801 \\
(0.488)\end{array}$ & & $\begin{array}{c}0.342 \\
(0.521)\end{array}$ \\
\hline Ambiguity aversion & & $\begin{array}{c}-1.463 * * * \\
(0.522)\end{array}$ & $\begin{array}{c}-1.316^{* *} \\
(0.559)\end{array}$ & & $\begin{array}{c}-1.298 * * * \\
(0.447)\end{array}$ & $\begin{array}{c}-1.186 * * \\
(0.478)\end{array}$ \\
\hline Age & & & & $\begin{array}{c}0.144 * * * \\
(0.045)\end{array}$ & $\begin{array}{c}0.139 * * * \\
(0.045)\end{array}$ & $\begin{array}{c}0.139 * * * \\
(0.045)\end{array}$ \\
\hline Sex & & & & $\begin{array}{l}-2.465 \\
(1.508)\end{array}$ & $\begin{array}{l}-2.373 \\
(1.502)\end{array}$ & $\begin{array}{l}-2.426 \\
(1.505)\end{array}$ \\
\hline Education & & & & $\begin{array}{c}1.524 * * * \\
(0.526)\end{array}$ & $\begin{array}{c}1.474 * * * \\
(0.524)\end{array}$ & $\begin{array}{c}1.486^{* * *} \\
(0.525)\end{array}$ \\
\hline Dependency ratio & & & & $\begin{array}{l}-2.120 \\
(2.467)\end{array}$ & $\begin{array}{l}-2.183 \\
(2.461)\end{array}$ & $\begin{array}{l}-2.227 \\
(2.462)\end{array}$ \\
\hline Time preference & & & & $\begin{array}{l}-3.341 \\
(2.282)\end{array}$ & $\begin{array}{c}-4.036^{*} \\
(2.295)\end{array}$ & $\begin{array}{c}-4.121^{*} \\
(2.299)\end{array}$ \\
\hline Trust in the insurer & & & & $\begin{array}{c}8.606 * * * \\
(1.564)\end{array}$ & $\begin{array}{c}8.939 * * * \\
(1.560)\end{array}$ & $\begin{array}{c}8.872 * * * \\
(1.564)\end{array}$ \\
\hline IBI promotion & & & & $\begin{array}{c}10.681 * * * \\
(1.397)\end{array}$ & $\begin{array}{c}10.554 * * * \\
(1.395)\end{array}$ & $\begin{array}{c}10.551 * * * \\
(1.395)\end{array}$ \\
\hline Peer influence & & & & $\begin{array}{c}7.276^{* * * *} \\
(1.175)\end{array}$ & $\begin{array}{c}7.079 * * * \\
(1.173)\end{array}$ & $\begin{array}{c}7.107 * * * \\
(1.174)\end{array}$ \\
\hline IAS & & & & $\begin{array}{c}3.596 * * * \\
(0.993)\end{array}$ & $\begin{array}{c}3.548 * * * \\
(0.990)\end{array}$ & $\begin{array}{c}3.568 * * * \\
(0.991)\end{array}$ \\
\hline Land size in qarxi & & & & $\begin{array}{l}0.104^{*} \\
(0.059)\end{array}$ & $\begin{array}{l}0.112^{*} \\
(0.059)\end{array}$ & $\begin{array}{l}0.112^{*} \\
(0.059)\end{array}$ \\
\hline Livestock size (TLU) & & & & $\begin{array}{c}0.157 * * \\
(0.063)\end{array}$ & $\begin{array}{c}0.160 * * \\
(0.062)\end{array}$ & $\begin{array}{c}0.159 * * \\
(0.063)\end{array}$ \\
\hline Distance from market & & & & $\begin{array}{c}1.679 * * * \\
(0.520)\end{array}$ & $\begin{array}{c}1.625^{* * *} \\
(0.519)\end{array}$ & $\begin{array}{c}1.638 * * * \\
(0.519)\end{array}$ \\
\hline Extension contact & & & & $\begin{array}{l}4.148 * * \\
(2.029)\end{array}$ & $\begin{array}{l}4.180 * * \\
(2.024)\end{array}$ & $\begin{array}{l}4.162 * * \\
(2.024)\end{array}$ \\
\hline CPS zone & & & & $\begin{array}{c}0.021 \\
(0.094)\end{array}$ & $\begin{array}{c}0.015 \\
(0.094)\end{array}$ & $\begin{array}{c}0.016 \\
(0.094)\end{array}$ \\
\hline District 1 & & & & $\begin{array}{l}3.215^{*} \\
(1.881)\end{array}$ & $\begin{array}{c}3.086 \\
(1.876)\end{array}$ & $\begin{array}{l}3.118^{*} \\
(1.878)\end{array}$ \\
\hline District 2 & & & & $\begin{array}{l}-1.157 \\
(1.515)\end{array}$ & $\begin{array}{l}-1.047 \\
(1.510)\end{array}$ & $\begin{array}{l}-1.088 \\
(1.512)\end{array}$ \\
\hline Constant & $\begin{array}{c}19.098 * * * \\
(0.585)\end{array}$ & $\begin{array}{c}18.827 * * * \\
(0.596)\end{array}$ & $\begin{array}{c}18.800 * * * \\
(0.598)\end{array}$ & $\begin{array}{c}-16.367 * * * \\
(4.252)\end{array}$ & $\begin{array}{c}-16.175 * * * \\
(4.242)\end{array}$ & $\begin{array}{c}-16.105 * * * \\
(4.244)\end{array}$ \\
\hline Observations & 1,139 & 1,139 & 1,139 & 1,139 & 1,139 & 1,139 \\
\hline R-squared & 0.002 & 0.007 & 0.007 & 0.308 & 0.311 & 0.311 \\
\hline
\end{tabular}

Note: ${ }^{* * *} \mathrm{p}<0.01,{ }^{* *} \mathrm{p}<0.05,{ }^{*} \mathrm{p}<0.1$. Standard errors are in parentheses. Regressions in Table 2.4 are undertaken using OLS. The dependent variable is the number of months that the household have been adopting IBI over the period 2013-2017.

\subsubsection{Impacts of risk and ambiguity aversion on late, dropout and persistent IBI adoption decisions}

Next, Table 2.5 presents estimated results of the impacts of risk and ambiguity aversion on late, dropout and persistent IBI adoption decisions. It shows that risk aversion is positively associated with late adoption decision without covariates as well as with inclusion of a set of covariates while ambiguity aversion has no a statistically significant impact on late adoption decisions at 5 percent level (see columns 1-4). 
Table 2.5: Heterogeneous effects of risk and ambiguity aversion on categories of adopters

\begin{tabular}{|c|c|c|c|c|c|c|c|c|c|c|c|c|}
\hline \multirow[b]{2}{*}{ Variables } & \multicolumn{4}{|c|}{ Late adopters } & \multicolumn{4}{|c|}{ Dropouts } & \multicolumn{4}{|c|}{ Persistent adopters } \\
\hline & (1) & (2) & (3) & (4) & (5) & (6) & (7) & (8) & (9) & (10) & (11) & (12) \\
\hline Risk aversion & $0.334 * * *$ & $0.255^{* * *}$ & & $0.304 * * *$ & $-0.186^{* *}$ & $-0.187 * *$ & & $-0.253 * * *$ & 0.092 & $0.140^{*}$ & & 0.095 \\
\hline & $(0.078)$ & $(0.079)$ & & $(0.084)$ & $(0.080)$ & $(0.080)$ & & $(0.087)$ & $(0.089)$ & $(0.085)$ & & $(0.092)$ \\
\hline \multirow[t]{2}{*}{ Ambiguity aversion } & $0.170 * *$ & & 0.049 & $0.147 *$ & $-0.176^{* * *}$ & & -0.089 & $-0.166^{* *}$ & 0.082 & & $-0.15^{*}$ & -0.120 \\
\hline & $(0.081)$ & & $(0.078)$ & $(0.085)$ & $(0.067)$ & & $(0.070)$ & $(0.075)$ & $(0.084)$ & & $(0.08)$ & $(0.090)$ \\
\hline \multirow[t]{2}{*}{ Age } & & 0.009 & 0.010 & 0.010 & & 0.011 & 0.010 & 0.010 & & 0.005 & 0.01 & 0.005 \\
\hline & & $(0.008)$ & $(0.008)$ & $(0.008)$ & & $(0.007)$ & $(0.007)$ & $(0.007)$ & & $(0.009)$ & $(0.01)$ & $(0.009)$ \\
\hline \multirow[t]{2}{*}{ Sex } & & $0.515^{*}$ & $0.553^{*}$ & $0.517^{*}$ & & -0.307 & -0.332 & -0.305 & & -0.245 & -0.23 & -0.240 \\
\hline & & $(0.285)$ & $(0.283)$ & $(0.285)$ & & $(0.231)$ & $(0.231)$ & $(0.232)$ & & $(0.297)$ & $(0.30)$ & $(0.298)$ \\
\hline \multirow[t]{2}{*}{ Education } & & 0.019 & 0.012 & 0.022 & & 0.053 & 0.060 & 0.049 & & $0.218 * *$ & $0.21 * *$ & $0.216^{* *}$ \\
\hline & & $(0.091)$ & $(0.090)$ & $(0.091)$ & & $(0.080)$ & $(0.080)$ & $(0.080)$ & & $(0.099)$ & $(0.10)$ & $(0.099)$ \\
\hline \multirow[t]{2}{*}{ Dependency ratio } & & 0.460 & 0.515 & 0.488 & & -0.203 & -0.243 & -0.215 & & -0.467 & -0.47 & -0.485 \\
\hline & & $(0.443)$ & $(0.441)$ & $(0.444)$ & & $(0.390)$ & $(0.389)$ & $(0.392)$ & & $(0.478)$ & $(0.478)$ & $(0.479)$ \\
\hline \multirow[t]{2}{*}{ Time preference } & & -0.377 & -0.212 & -0.304 & & $0.729 * *$ & $0.578^{*}$ & $0.622 *$ & & $-1.765^{* * *}$ & $-1.85^{* * *}$ & $-1.847 * * *$ \\
\hline & & $(0.413)$ & $(0.410)$ & $(0.418)$ & & $(0.341)$ & $(0.343)$ & $(0.345)$ & & $(0.506)$ & $(0.51)$ & $(0.508)$ \\
\hline \multirow[t]{2}{*}{ Trust in the insurer } & & $1.229 * * *$ & $1.246 * * *$ & $1.200 * * *$ & & $1.097 * * *$ & $1.086 * * *$ & $1.141 * * *$ & & $1.169 * * *$ & $1.230 * * *$ & $1.210 * * *$ \\
\hline & & $(0.371)$ & $(0.371)$ & $(0.372)$ & & $(0.356)$ & $(0.354)$ & $(0.357)$ & & $(0.432)$ & $(0.433)$ & $(0.434)$ \\
\hline \multirow[t]{2}{*}{ IBI promotion } & & 0.424 & $0.426^{*}$ & $0.442 *$ & & $1.266^{* * *}$ & $1.242 * * *$ & $1.256 * * *$ & & $0.570^{*}$ & $0.548^{*}$ & $0.545^{*}$ \\
\hline & & $(0.259)$ & $(0.259)$ & $(0.260)$ & & $(0.274)$ & $(0.272)$ & $(0.274)$ & & $(0.303)$ & $(0.303)$ & $(0.303)$ \\
\hline \multirow[t]{2}{*}{ Peer influence } & & -0.129 & -0.130 & -0.102 & & $0.830 * * *$ & $0.815^{* * *}$ & $0.799 * * *$ & & $0.669 * *$ & $0.646^{* *}$ & $0.653 * *$ \\
\hline & & $(0.207)$ & $(0.206)$ & $(0.207)$ & & $(0.213)$ & $(0.213)$ & $(0.214)$ & & $(0.274)$ & $(0.275)$ & $(0.275)$ \\
\hline \multirow[t]{2}{*}{ IAS } & & -0.021 & -0.022 & -0.018 & & $0.266^{*}$ & $0.261 *$ & $0.263^{*}$ & & $0.349 *$ & $0.345^{*}$ & $0.348 *$ \\
\hline & & $(0.170)$ & $(0.169)$ & $(0.171)$ & & $(0.153)$ & $(0.153)$ & $(0.154)$ & & $(0.188)$ & $(0.188)$ & $(0.188)$ \\
\hline \multirow[t]{2}{*}{ Land size in qarxi } & & 0.014 & 0.012 & 0.013 & & 0.003 & 0.004 & 0.004 & & 0.007 & 0.007 & 0.008 \\
\hline & & $(0.009)$ & $(0.009)$ & $(0.009)$ & & $(0.008)$ & $(0.008)$ & $(0.008)$ & & $(0.010)$ & $(0.010)$ & $(0.010)$ \\
\hline \multirow[t]{2}{*}{ Livestock size (TLU) } & & 0.011 & 0.012 & 0.011 & & 0.012 & 0.010 & 0.012 & & 0.011 & 0.012 & 0.011 \\
\hline & & $(0.010)$ & $(0.010)$ & $(0.010)$ & & $(0.009)$ & $(0.009)$ & $(0.009)$ & & $(0.010)$ & $(0.010)$ & $(0.010)$ \\
\hline \multirow[t]{2}{*}{ Distance from market } & & $-0.215^{* *}$ & $-0.207 * *$ & $-0.208^{* *}$ & & $0.198 * * *$ & $0.193 * *$ & $0.193 * *$ & & 0.067 & 0.069 & 0.066 \\
\hline & & $(0.094)$ & $(0.093)$ & $(0.094)$ & & $(0.076)$ & $(0.076)$ & $(0.076)$ & & $(0.093)$ & $(0.093)$ & $(0.093)$ \\
\hline \multirow[t]{2}{*}{ Extension contact } & & -0.253 & -0.243 & -0.254 & & 0.275 & 0.233 & 0.260 & & $2.258 * *$ & $2.262 * *$ & $2.252 * *$ \\
\hline & & $(0.336)$ & $(0.334)$ & $(0.337)$ & & $(0.352)$ & $(0.350)$ & $(0.351)$ & & $(1.019)$ & (1.019) & $(1.019)$ \\
\hline \multirow[t]{2}{*}{ CPS Zone } & & $-0.050 * * *$ & $-0.050 * * *$ & $-0.050 * * *$ & & 0.013 & 0.014 & 0.013 & & 0.014 & 0.014 & 0.014 \\
\hline & & $(0.018)$ & $(0.018)$ & $(0.018)$ & & $(0.015)$ & $(0.015)$ & $(0.015)$ & & $(0.018)$ & $(0.018)$ & $(0.018)$ \\
\hline \multirow[t]{2}{*}{ District 1} & & $-1.636^{* * *}$ & $-1.627 * * *$ & $-1.636^{* * *}$ & & $0.595 * *$ & $0.610 * *$ & $0.607 * *$ & & 0.158 & 0.167 & 0.164 \\
\hline & & $(0.361)$ & $(0.360)$ & $(0.361)$ & & $(0.288)$ & $(0.287)$ & $(0.288)$ & & $(0.326)$ & $(0.326)$ & $(0.326)$ \\
\hline \multirow[t]{2}{*}{ District 2} & & $-0.730 * * *$ & $-0.682 * * *$ & $-0.747 * * *$ & & $0.481 * *$ & $0.452 *$ & $0.497 * *$ & & -0.314 & -0.295 & -0.307 \\
\hline & & $(0.252)$ & $(0.250)$ & $(0.253)$ & & $(0.232)$ & $(0.231)$ & $(0.232)$ & & & $(0.260)$ & $(0.261)$ \\
\hline \multirow[t]{2}{*}{ Constant } & $-1.67 * * *$ & $-2.44 * * *$ & $-2.54 * * *$ & $-2.48 * * *$ & $-1.25 * * *$ & $-5.62 * * *$ & $-5.47 * * *$ & $-5.60 * * *$ & $-1.94 * * *$ & $-6.47 * * *$ & $-6.49 * * *$ & $-6.46^{* * *}$ \\
\hline & $(0.09)$ & $(0.794)$ & $(0.790)$ & $(0.797)$ & $(0.074)$ & $(0.770)$ & $(0.766)$ & $(0.771)$ & $(0.094)$ & $(1.313)$ & $(1.312)$ & $(1.313)$ \\
\hline Observations & 1,139 & 1,139 & 1,139 & 1,139 & 1,139 & 1,139 & 1,139 & 1,139 & 1,139 & 1,139 & 1,139 & 1,139 \\
\hline
\end{tabular}

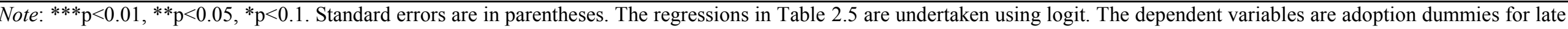

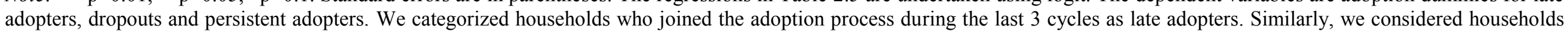
who dropped the adoption for at least 3 cycles as dropouts, and those who did not dropout for more than 3 cycles as persistent adopters. 
Table 2.5 also indicates that an increase in risk aversion as well as an increase in ambiguity aversion significantly decreases the rate of dropout for households who have adopted IBI (see columns 5-8). Thus, from the prespective of sustained uptake, both risk and ambiguity aversion behaviour of smallholders matter for their dropout decisions once they enaged in IBI adoption. Further, Table 2.5 also reveals that risk aversion as well as ambiguity aversion has insignificant effect on persistent adoption decisions of the households at 5 percent level (see columns 9-12). While ambiguity about the IBI contract is potentially high in the initial periods of adoption due to inadequate information, such effect can reduce overtime due to learning. To this end, though the level of literacy of smallholders is low in the study area, the insurance firm (OIC) offered different training and awareness creation activities through which learning, experience sharing or information diffusion can be effected. Thus, with increased information and understanding about IBI, the ambiguity of households might have been clarified overtime.

Some control variables were observed to influence IBI uptake decisions of the smallholders. Age and education of the household head, trust in the insurer, IBI poduct promotion and intertemporal adverse selection postitively affect IBI adoption decisions. Larger livestock size and frequent contact with extension agents were also evidenced to enhance IBI adoption. Trust in the insurer, distance from the market and crop production zone were also influenced late adoption decisions. Similarly, trust in the insurer, peer influence, distance from the market and IBI product promotion also affect dropout decisions of the households. Level of education, time preference, trust in the insurer and peer influence again determined persistent adoption decisions. This seems to be a promising avenue for future investigation with a larger panel dataset. Although we do control for many variables, there is still a potential for omitted variables. For instance, we might think that people who are more well-connected in social networks may likely adopt IBI and they may also tend to be less ambiguity-averse (Munshi 2004). But we do not have data on social networks. Experimental data on social networks would have further strengthened our findings.

\subsection{Conclusion}

Using data from lab-in-the field experiments and household survey, this study examines whether risk and/or ambiguity aversion can explain the low uptake of IBI by smallholders in Ethiopia. Though risk-averse smallholders attempt to insure against stochastic production risks, the main problem in adoption of IBI is the ambiguity due to basis risk. Because of a higher potential degree of ambiguity associated with the failure of the IBI contract to 
accurately reflect loss realizations of smallholders, we hypothesized that, on top of risk aversion, ambiguity aversion might play a large role in hampering adoption of IBI in the study area. First, we tested the effects of risk and ambiguity aversion on incidence of IBI adoption using a discrete choice adoption model. Our analyses suggest that an increase in risk aversion increases while an increase in ambiguity aversion reduces the probability of IBI adoption among the smallholders. In contrast with most of the previous empirical tests of the roles of risk aversion on technology adoption, we find that risk aversion speeds-up rather than delays the adoption of IBI. This difference may be due to the fact that most empirical literature so far has been considering the whole uncertainty as risk in their analysis, without having a split look at risk and ambiguity that sums up to uncertainty. Second, we estimated an OLS model to measure the effect of risk and ambiguity aversion on intensity of IBI adoption, measured by the number of months that households have stayed in adoption phase. Results reveal that risk aversion increases while ambiguity aversion decreases the number of months that the smallholders have stayed in IBI adoption over the period 2013-2017. Thirdly, we estimated the differential effects of risk and ambiguity aversion on late, dropout and persistent adoption decisions of the smallholders. This analysis is important from the perspective of sustained uptake. Our results suggest that risk aversion increases while ambiguity aversion has no effect on late adoption decisions. Both risk and ambiguity aversions also decrease dropout decisions once the households adopted IBI. However, our results evidence that neither risk aversion nor ambiguity aversion has a significant effect on persistent IBI adoption decisions of the households.

There is interplay between risk and ambiguity aversion in IBI adoption decisions of smallholders. Since farmers are averse to production risk, most of them can be fundamentally willing to insure such risk using the IBI contract. However, in the presence of basis risk, farmers can remain ambiguous about whether the contract accurately reveals their actual loss realizations. This means, though risk-averse smallholders are willing to buy IBI in order to hedge their production risk, their actual uptake may not be effective due to the ambiguity surrounding the validity of the contract to payout in the future. Ambiguity aversion thus dictates uptake decisions of smallholders in a sort of dominance effect. However, the effect of ambiguity aversion on IBI adoption diminishes overtime, because farmers' learning and experience in adoption gradually clarifies their ambiguity regarding the nature of the contract. In addition, improvements in IBI design that inherently reduces basis risk helps to reduce the ambiguity surrounding the validity of IBI contract overtime. 
Several implications for future studies on technology adoption can be drawn from this study. The first is the need to distinguish between risk and ambiguity aversion behaviour in analysis of technology adoption decisions. Second, the effects of risk and ambiguity aversion on technology adoption can vary over time. These implications underscore the need for future studies to theoretically distinguish and empirically test the relative relevance of the effect of risk and ambiguity aversion in technology adoption decisions. Our study also has methodological implications in designing studies related to the adoption of rural technologies. Combining experimental methods to measure variables that are otherwise difficult to be identified like risk and ambiguity aversion with survey methods helps to better understand the role of the behaviour of decision making units. In addition, the fact that the effect of ambiguity aversion on IBI adoption diminishes overtime suggests the importance of learning mechanisms such as training and information sharing in shaping the behaviour and capacity of individual smallholders to manage uncertainty through adoption of new technologies. Since large numbers of rural producers and entrepreneurs inherently face challenges of managing uncertainty in an increasingly volatile global economy, the imperative to deepen our understanding in this regard seems high.

\section{Appendix 1: Risk and ambiguity aversion experiments}

\section{Description}

Enumerators explain to the respondent that, we will pay you ETB 15 for the time that you spend with us today. In addition, if you are willing to participate in the following two games, then you will earn some additional money based on your chances of winning. The two games have separate payments based on your chance of winning. In the first game, you are able to win an additional amount of Birr 20. The same holds true for the second game.

\section{Appendix 1A: Ambiguity aversion experiment}

Enumerators explain to the respondent that we have one game that pays you some amount of money based on your chances of drawing a blue pen. This game involves 11 decisions. You need to respond to all the 11 questions. To play this game, there are two bags from which you can draw a blue pen: Bag 1 and Bag 2. Each bag contains 10 pens, some blue and some red. In bag 1 , the bag on the right hand side of the enumerator, the number of blue and red pens is known for each of the 11 decisions. Thus, the proportion of red and blue pens is known for bag 1. But in Bag 2, the bag on the left hand side of the enumerator, while the total number of 
pens is known, the number of blue or red pens is unknown. You earn Birr 20 if you draw a 'Blue' pen and earn nothing if you draw a red one. Are you willing to participate? If yes, here is the game.

\section{Question 1: Which bag do you prefer? (Tick the relevant box)}

Bag 1: contains 10 blue pens and no red pen.

Bag 2: contains 10 pens. But how many blue and how many red are there is unknown.

\section{Question 2: Which bag do you prefer? (Tick the relevant box)}

Bag 1: contains 9 blue pens and only 1 red pen.

Bag 2: contains 10 pens. But how many blue and how many red are there is unknown.

Question 3: Which bag do you prefer? (Tick the relevant box)

Bag 1: contains 8 blue pens and 2 red pens

Bag 2: contains 10 pens. But how many blue and how many red are there is unknown.

Question 4: Which bag do you prefer? (Tick the relevant box)

Bag 1: contains 7 blue pens and 3 red pens

Bag 2: contains 10 pens. But how many blue and how many red are there is unknown.

Question 5: Which bag do you prefer? (Tick the relevant box)

Bag 1: contains 6 blue pens and 4 red pens

Bag 2: contains 10 pens. But how many blue and how many red are there is unknown.

Question 6: Which bag do you prefer? (Tick the relevant box)

Bag 1: contains 5 blue pens and 5 red pens

Bag 2: contains 10 pens. But how many blue and how many red are there is unknown.

Question 7: Which bag do you prefer? (Tick the relevant box)

Bag 1: contains 4 blue pens and 6 red pens

Bag 2: contains 10 pens. But how many blue and how many red are there is unknown.

Question 8: Which bag do you prefer? (Tick the relevant box)

Bag 1: contains 3 blue pens and 7 red pens

Bag 2: contains 10 pens. But how many blue and how many red are there is unknown.

Question 9: Which bag do you prefer? (Tick the relevant box) 
Bag 1: contains 2 blue pens and 8 red pens

Bag 2: contains 10 pens. But how many blue and how many red are there is unknown.

Question 10: Which bag do you prefer? (Tick the relevant box)

Bag 1: contains 1 blue pen and 9 red pens.

Bag 2: contains 10 pens. But how many blue and how many red are there is unknown.

Question 11: Which bag do you prefer? (Tick the relevant box)

Bag 1: contains no blue pen and 10 red pens

Bag 2: contains 10 pens. But how many blue and how many red are there is unknown.

\section{Appendix 1B: Risk aversion experiment}

Enumerators explain to the respondent that we have also one additional game that pays you some amount of money based on your choices. This game involves 11 decisions. You need to answer all 11 questions. To play this game, there are two lotteries: Lottery A and Lottery B. Lottery A offers a sure pay-off that varies across the 11 decisions. But lottery B offers an option with two alternatives with a 50-50 probability. If you choose Lottery B, you will toss a coin. If the coin is 'Head', you get Birr 20; if it is 'Tail', you get nothing. Are you willing to participate? If yes, here is the game.

Question 1: Which option do you choose? (Tick the relevant box)

Lottery A: You will get Birr 18 for sure

Lottery B: Flip a coin: If it is 'Head', you get Birr 20; if it is 'Tail', you get Birr 0.

Question 2: Which option do you choose? (Tick the relevant box)

Lottery A: You will get Birr 16 for sure

Lottery B: Flip a coin: If it is 'Head', you get Birr 20; if it is 'Tail', you get Birr 0.

Question 3: Which option do you choose? (Tick the relevant box)

Lottery A: You will get Birr 14 for sure

Lottery B: Flip a coin: If it is 'Head', you get Birr 20; if it is 'Tail', you get Birr 0.

Question 4: Which option do you choose? (Tick the relevant box)

Lottery A: You will get Birr 12 for sure

Lottery B: Flip a coin: If it is 'Head', you get Birr 20; if it is 'Tail', you get Birr 0. 
Question 5: Which option do you choose? (Tick the relevant box)

Lottery A: You will get Birr 10 for sure

Lottery B: Flip a coin: If it is 'Head', you get Birr 20; if it is 'Tail', you get Birr 0.

Question 6: Which option do you choose? (Tick the relevant box)

Lottery A: You will get Birr 8 for sure

Lottery B: Flip a coin: If it is 'Head', you get Birr 20; if it is 'Tail', you get Birr 0.

Question 7: Which option do you choose? (Tick the relevant box)

Lottery A: You will get Birr 6 for sure

Lottery B: Flip a coin: If it is 'Head', you get Birr 20; if it is 'Tail', you get Birr 0.

Question 8: Which option do you choose? (Tick the relevant box)

Lottery A: You will get Birr 4 for sure

Lottery B: Flip a coin: If it is 'Head', you get Birr 20; if it is 'Tail', you get Birr 0.

Question 9: Which option do you choose? (Tick the relevant box)

Lottery A: You will get Birr 2 for sure

Lottery B: Flip a coin: If it is 'Head', you get Birr 20; if it is 'Tail', you get Birr 0.

Question 10: Which option do you choose? (Tick the relevant box)

Lottery A: You will get Birr 1 for sure

Lottery B: Flip a coin: If it is 'Head', you get Birr 20; if it is 'Tail', you get Birr 0.

Question 11: Which option do you choose? (Tick the relevant box)

Lottery A: You will get nothing for sure

Lottery B: Flip a coin: If it is 'Head', you get Birr 20; if it is 'Tail', you get Birr 0. 


\title{
Chapter 3
}

\section{Trust, liquidity constraints and the adoption of weather insurance: A randomized controlled trial in Ethiopia}

\begin{abstract}
We report the results of a drought insurance experiment in Ethiopia, and examine whether uptake of index-based insurance is enhanced if we allow farmers to pay after harvest, addressing a liquidity constraint. We also test to what extent uptake can be enhanced by channeling insurance via indigenous risk-sharing institutions (Iddirs) that bear altruistic trust. The insurance product with delayed premium increases uptake substantially when compared to standard insurance, and promoting this new product via Iddirs results in even greater uptake. However, default rates associated with delayed payments are relatively high and concentrated in a small number of Iddirs - compromising the economic viability of the novel product. We examine how default rates can be reduced.
\end{abstract}

Keywords: index-based insurance; liquidity constraints; trust; randomized controlled trial

This chapter is based on:

Belissa, Bulte, Cecchi, Gangopadhyay and Lensink (2018): Trust, liquidity constraints and the adoption of weather insurance: A randomized controlled trial in Ethiopia. Under review, Journal of Development Economics 


\subsection{Introduction}

The majority of the world's poor reside in rural areas and their economic fate depends crucially on the performance of the agricultural sector (Ray 1998; Jalan and Ravallion 1999; World Bank 2007; Haggblade et al. 2007; Christiaensen et al. 2011). Despite global efforts to promote the intensification of rain-fed agriculture, the diffusion of modern agricultural technologies - such as improved crop varieties and fertilizer - remains low (Lamb 2003; Barrett et al. 2017). Evidence is growing that downside (production) risk is an important factor impeding the uptake of these technologies (Emerick et al. 2016). ${ }^{7}$ Purchasing external inputs in a context where harvests may fail is risky - exposing farmers to the risk of heavy debts and the loss of valuable assets (Boucher et al. 2008). The pursuit of 'low-risk lowreturn' activities may be rational in such a context (Walker and Ryan 1990). Increasing the uptake of insurance against weather shocks in rain-fed production systems may therefore be an important component of strategies to modernize agriculture and lift farm households out of poverty (Sarris 2013; Mobarak and Rosenzweig 2013; Karlan et al. 2014; Cai 2016; Elabed and Carter 2016).

In recent years, experiments with index-insurance products have sought to overcome wellknown problems associated with indemnity-based insurance. These include prohibitive transaction costs, asymmetric information and moral hazard as well as covariate shocks that are hard to re-insure. Index-insurance delinks payouts from farm-level losses, and allows farmers to purchase coverage based on an index correlated with these losses. This may be a measure of average biomass productivity or a measure of local rainfall during a certain time period or a measure of vegetative cover on the earth surface - variables that are objectively quantifiable and verifiable. Payouts are triggered when the index falls short of a predetermined threshold.

While index insurance can promote agricultural intensification, challenges for development remain because adoption of index insurance is low, hovering below 10\% (Cole et al. 2013). The literature identifies several reasons for low uptake of index insurance. For example, index insurance provides only imperfect coverage for household shocks if individual

\footnotetext{
${ }^{7}$ Various other factors also play a role in explaining slow diffusion of new technologies. These include heterogeneity in (net) benefits and profitability (Suri 2011), under-performing extension systems, and lack of liquidity (including lack of access to credit). For a recent overview, refer to Foster and Rosenzweig (2010).
} 
damages are not correlated with the index. If the index is not identical to on-farm losses, residual risk (or basis risk) remains. Individual losses may be high while the index does not reach the threshold, implying that insured farmers are worse off than in the absence of insurance because they paid the premium (Clarke 2016). 'False negatives' undermine the expected utility of adoption, especially for highly risk averse farmers. The combination of uncertain rainfall and uncertain payouts implies that farmers face a compound lottery, inviting ambiguity aversion (Elabed and Carter 2015; Belissa et al. 2018a). Interventions that aimed to reduce basis risk indeed help to increase uptake (Elabed et al. 2013).

In this chapter we report on the outcomes of an RCT in rural Ethiopia that focused on two alternative reasons for low adoption of insurance: ${ }^{8}$ (i) lack of liquidity to make upfront insurance premium payment, and (ii) lack of trust in the insurance product (or insurance provider). Consider the former first. To study the role of liquidity constraints during the planting season, we allow (randomly selected) farmers to pay the premium after harvest. Many smallholders are unable to mobilize the resources needed to pay for payment of the premium upfront. ${ }^{9}$ The standard insurance product requires farmers to pay the premium when disposable income is at its lowest and the marginal utility of cash is at its highest - just before the 'hunger season'. In return, they might receive compensation after harvest when, no matter how meagre, disposable income is often higher than in the planting season. We allow smallholders to postpone premium payment until after the harvest, and henceforth call this insurance product 'I owe you (IOU)'. The properties of the IOU, except for the delayed payment, are identical to those of a standard product, but the delayed premium is slightly higher to account for the opportunity cost of time (making the premiums of both products inter-temporally equivalent). A crucial challenge for the viability of delayed payment schemes is default after production uncertainty has been resolved in case there was no payout. Defaults can also be induced due to basis risk. We probe this issue by exploring contracts and leveraging group dynamics or peer pressure to manage defaults.

\footnotetext{
${ }^{8}$ Other reasons for imperfect uptake exist. For example, the fact that insurance products are 'complex' and low levels of financial literacy among target populations imply that not all potential beneficiaries can comprehend its logic or understand the potential benefits (Cole et al. 2013; Cai et al. 2015). Lack of experience with shocks may also matter as does precise knowledge about the probability of disasters (Cai and Song 2017).

${ }^{9}$ Such outcomes may be due to several factors, including poverty gap dynamics and present bias (hyperbolic discounting leading to procrastination - see Laibson 1997; Duflo et al. 2011).
} 
Second, to understand the role of trust in insurance uptake decisions, we vary the marketing channel between the cooperatives (statutory channel that bears low level of trust at the public due to state control and bureaucratic operations) and Iddirs (customary institutions that bear higher altruistic trust at the public). ${ }^{10}$ Iddirs are indigenous risk-sharing institutions in Ethiopia, originally created to help their members to organize burial ceremonies (Aredo 1993), but currently Iddirs in Ethiopia are often engaged in a broader spectrum of activities and mutual assistances (Aredo 2010). In this experiment, we trained Iddir leaders about the benefits of index insurance, and encouraged them to train their members. It is important to emphasize that insurance was sold to individual members directly from the insurance firm, Oromia Insurance Company (OIC). We did not sell insurance to Iddirs. Rather we used the customary Iddir channel for promotion of insurance. The idea is since people trust their Iddir, they can also trust the benefits of insurance, when advertised or promoted by via Iddirs. This in turn can potentially increase the uptake of insurance. In the conventional way, similar to other agricultural technologies, a statutory channel of cooperatives was used to promote the insurance technology in Ethiopia.

Our study in this chapter is closely related to the following two papers. First, Dercon et al. (2014) propose selling index insurance to Iddirs because there might be important coordination benefits from group-wise purchasing of index insurance - in the presence of basis risk, formal and informal insurance may be complements (see also De Janvry et al. 2014). ${ }^{11}$ They evaluate the impact of an intervention that trains Iddir members to benefit from post-payout redistribution, and find that such training increases the uptake of insurance. Second, Casaburi and Wills (2019) study delayed payments of the premium to induce

\footnotetext{
${ }^{10}$ Iddirs are indigenous mutual help associations made up by a group of persons united by ties of family and friendship, living or working together, or belonging to the same ethnic group. Their member size, composition, function and organization can differ from one to another. Iddirs are based on voluntary mutual agreements and intense participation of their members. Such risk-sharing networks provide reciprocity based assistances when members face shocks, and thus members have high degrees of altruistic trust on Iddirs (Aredo 1993:2010; Fehr and Fischbacher 2003; Bernhard et al. 2006; Dercon et al. 2006; Fafchamps and Gubert 2007; Della et al. 2012).

${ }^{11}$ This enables group members to redistribute payouts among each other. Since members have superior knowledge about true damages, this may reduce basis risk. Observe that traditional indemnity-based insurance typically serves as a substitute mechanism for informal risk-sharing arrangements (Arnott and Stiglitz 1991).
} 
insurance uptake, but the insurance is interlinked with a contract farming scheme (which prevents defaults on the premium payment commitments). They find that uptake increases to $72 \%$, compared to $5 \%$ for the standard contract. It is an open question whether this result extends to other contracting arrangements, because most smallholders are not engaged in contract farming (Oya 2012).

We use a factorial design involving 144 Iddirs and 8,579 member households to test whether delayed premium payments and the promotion of insurance via Iddirs affect adoption of index insurance. We test for 'level effects' and complementarities among trust, delayed premium payment and contracts. Our main results are that the IOU has a large accentuating effect on uptake when introduced in isolation. However, the defaults rates are higher for IOUs sold without the involvement of Iddirs, which might jeopardize the viability of the scheme. Promoting standard insurance via Iddirs does not significantly increase adoption. Hence, the combination of IOU and Iddir outperforms all other modalities. We conclude that a package of measures - combining the delayed payment option with promotion through Iddirs and measures to curb default using contracts - hold promise for scaling up.

The remainder of this chapter is organized as follows. Section 3.2 presents a theoretical model that provides a framework for the role of trust and liquidity constraints as well as the associated defaults and basis risk in adoption of weather insurance. We next seek to take these predictions to the data. In Section 3.3 we sketch the context, explain the intervention and randomization strategy, and introduce our data. We demonstrate that the randomization 'worked' in the sense that we have created well-balanced experimental arms. Section 3.4 presents the results, considering both the uptake of insurance and default on repayment of premiums. Section 3.5 presents robustness analyses followed by conclusions in Section 3.6.

\subsection{Theoretical model ${ }^{12}$}

We present a theoretical model that shows how delayed payment of premiums and increased trust in the insurance product affect uptake of insurance and strategic defaults. We first contrast the IOU product to a standard one, where farmers pay a premium before uncertainty is resolved. Consider farmer $i, i=1,2, . . n$, deriving utility in period $t, t=0,1,2, .$. from cashflow $z_{i t}$. We assume that $z_{i 0}$ is known but the future is uncertain and $z_{i t}=\tilde{y}_{i t}$ is the

\footnotetext{
${ }^{12}$ This section is the sole contribution of my promotor Prof. Robert Lensink and our co-author Prof. Shubahshis Gangopadhyay.
} 
stochastic cash flow in periods $t=1,2, .$. The farmer derives utility each period from his cash flow and utility is expressed as the certainty equivalent of the cash flow as follows:

$$
u_{i 0}=z_{i 0} \text { and } u_{i t}=\bar{z}_{i t}-\frac{1}{2} \rho_{i} \sigma_{z_{i t}}^{2}
$$

where $\bar{z}_{i t}$ is the expected cash flow in period $t, t=1,2, . ., \sigma_{z_{i t}}^{2}$ is the variance of $z_{i t}$ and $\rho_{i}$ is the parameter measuring constant absolute risk aversion of farmer $i$. Assume that income stream $\tilde{y}_{i t}$ has support $\left[\underline{y}_{i}, \bar{y}_{i}\right]$ and that $\tilde{y}_{i t}$, is independently and identically distributed over time with mean $\bar{y}_{i t}=\bar{y}_{i}$ and variance $\sigma_{\tilde{y}_{i t}}^{2}=\sigma_{y_{i}}^{2}$ for all $t=1,2, \ldots$ Let $F_{t}\left(\tilde{y}_{i t}\right)=F\left(\tilde{y}_{i}\right)$ be the distribution of $\tilde{y}_{i t}$. If the farmer has no insurance (or saving opportunities), lifetime utility of the farmer can be written as:

$$
\begin{aligned}
\underline{U}_{i} & =z_{i 0}+\sum_{t=1}^{\infty} \beta^{t}\left(\bar{z}_{i t}-\frac{1}{2} \rho_{i} \sigma_{z_{i t}}^{2}\right) \\
& =y_{i 0}+\sum_{t=1}^{\infty} \beta^{t}\left(\bar{y}_{i}-\frac{1}{2} \rho_{i} \sigma_{y_{i}}^{2}\right)=y_{i 0}+\frac{\beta}{1-\beta}\left(\bar{y}_{i}-\frac{1}{2} \rho_{i} \sigma_{y_{i}}^{2}\right)
\end{aligned}
$$

\subsubsection{Insurance}

Now let us introduce insurance in the model. Suppose the farmer can buy one-period insurance in year 0 . If farmer $i$ buys insurance in $t=0$, then, the farmer gets a utility of $y_{i 0}-c\left(y_{i 0}\right) \pi$ in period 0 , where $\pi$ is the premium paid for the insurance and $c$ denotes the marginal utility of money. Different farmers have different values of $c$. In particular, we assume that $c^{\prime}\left(y_{i 0}\right)<0$, i.e., the marginal utility of money falls with the cash flow. ${ }^{13} \mathrm{We}$ further assume that $\bar{y}_{i} \geq \pi$, i.e., there are states of income when it is feasible to buy insurance. Observe the possibility that there exists $y^{\prime}$ where the purchase of insurance is affordable, i.e., $y^{\prime}-\pi>0$, but 'too costly' from a utility perspective, i.e., $y^{\prime}-c\left(y^{\prime}\right) \pi<0$.

\footnotetext{
${ }^{13}$ One way to interpret this is as follows. If the farmer buys insurance in period 0 , his period 0 utility is given by $u_{i}\left(y_{i 0}-\pi\right)$ which, by Taylor's expansion can be approximated as $u\left(y_{i 0}\right)-u^{\prime}\left(y_{i 0}\right) \pi=$ $y_{i 0}-u^{\prime}\left(y_{i 0}\right) \pi$. We can interpret $u^{\prime}\left(y_{i 0}\right)$ as the marginal utility of money or, the liquidity cost of buying insurance for a farmer with cash, or liquidity, $y_{i 0}$. For ease of notation we are writing this as $c\left(y_{i 0}\right)$. Note that, for a risk-averse farmer, $u^{\prime \prime}()<$.0 implies $c^{\prime}\left(y_{i 0}\right)<0$. Given our utility expression $c($.$) is always greater than or equal to unity.$
} 
Also note that in any insurance mechanism, the farmer gets a payout from the insurance when $\tilde{y}$ is low and no payout when $\tilde{y}$ is high. If $\tilde{x}$ denotes the insurance payout, then $\tilde{x}$ and $\tilde{y}$ are negatively correlated. If the farmer buys insurance, then his cash flow in the next period is given by $\tilde{z}=\tilde{y}+\tilde{x}$. We consider insurance as an uncertain cash flow claim in the next period which can be bought in this period for a price of $\pi$ and a utility $\operatorname{cost}$ of $c(z) \pi$. Denote the variance of $\tilde{x}_{i}$ as $\sigma_{x_{i}}^{2}$ and its covariance with $\tilde{y}_{i}$ as $\sigma_{y_{i} x_{i}}$. Note that $\tilde{x}_{i t}=x\left(\tilde{y}_{i t}\right)$ such that $\tilde{z}_{i t} \equiv \tilde{y}_{i t}+\tilde{x}_{i t} \geq \tilde{y}_{i t}$, with strict inequality for low values of $\tilde{y}_{i t}$ and equality for high values of $\tilde{y}_{i t}\left(\right.$ when $\left.\tilde{x}_{i t}=0\right)$. In particular, we have $\tilde{z}_{i t} \in\left[\underline{z}_{i t}, \bar{z}_{i t}\right] \subset\left[\underline{z}_{i}, \bar{y}_{i}\right]$ with $\underline{z}_{i}=\underline{y}_{i}+x\left(\underline{y}_{i}\right)$ and $\bar{z}_{i t}=\bar{y}_{i}$. Hence, $\left[\underline{z}_{i}, \bar{y}_{i}\right] \subset\left[\underline{y}_{i}, \bar{y}_{i}\right]$.

A comparison of utility levels with and without insurance enables us to identify the condition under which insurance is purchased. Let $a_{i t} \in\{0,1\}$ denote the decision to buy insurance in period $t$. If the farmer buys insurance in period 0 , the farmer's utility of the first two periods is given as:

$$
y_{i 0}-c\left(y_{i 0}\right) \pi+\beta\left[\bar{y}_{i}+\bar{x}_{i}-\frac{1}{2} \rho_{i}\left(\sigma_{y_{i}}^{2}+\sigma_{x}^{2}+2 \sigma_{x y_{i}}\right)-E\left[a_{i 1} c\left(\tilde{z}_{i 1}\right) \pi\right]\right]
$$

While, if the farmer does not buy insurance, the farmer's utility function is given as:

$$
y_{i 0}+\beta\left(\bar{y}_{i}-\frac{1}{2} \rho_{i} \sigma_{y_{i}}^{2}-E\left[a_{i 1} c\left(\tilde{y}_{i 1}\right) \pi\right]\right)
$$

where $c\left(\tilde{y}_{i 1}\right) \pi$ is the utility cost of buying insurance in period 1 if insurance was not bought in period 0 . Since cash flows in period 1 after buying insurance in period 0 are not lower than cash flows in period 1 if insurance was not bought in period $0, c\left(\tilde{z}_{i 1}\right) \leq c\left(\tilde{y}_{i 1}\right)$ and, hence, $E\left[a_{i 1} c\left(\tilde{z}_{i 1}\right) \pi\right] \leq E\left[a_{i 1} c\left(\tilde{y}_{i 1}\right) \pi\right]$. Comparing the two expressions, it is immediate that buying insurance in period 0 is the preferred option if

$$
\beta\left[\bar{x}_{i}-\frac{1}{2} \rho_{i}\left(\sigma_{x_{i}}^{2}+2 \sigma_{y_{i} x_{i}}\right)\right] \geq c\left(y_{i 0}\right) \pi
$$

Generalizing beyond period 0 , we obtain the following condition: ${ }^{14}$

$$
\beta\left[\bar{x}_{i}-\frac{1}{2} \rho_{i}\left(\sigma_{x_{i}}^{2}+2 \sigma_{y_{i} x_{i}}\right)\right] \geq c\left(\underline{z}_{i}\right) \pi
$$

\footnotetext{
${ }^{14}$ The condition in (3.4) is a sufficient condition but not a necessary one. Because even when the condition in eq. (3.4) does not hold, but if $E\left[a_{i 1} c\left(\tilde{z}_{i 1}\right) \pi\right]<E\left[a_{i 1} c\left(\tilde{y}_{i 1}\right) \pi\right]$, then, the farmer may buy insurance in period 0 . However, since $a_{i t}$ is a choice variable, we stick to the sufficient condition.
} 
This condition guarantees that if the insurance is bought in any period, it pays to buy insurance in the next period. ${ }^{15}$ Insurance is then a sustainable instrument in the sense that if bought once, it can be bought in the next period also.

\subsubsection{IOU insurance}

Now suppose the farmer has access to the IOU insurance which has the same payout plan as the erstwhile insurance but its premium can be paid in the same period as the payouts are made. The delayed premium payment equals $\pi(1+r)$ where $r$ is the risk-free interest rate that the insurance company could get on its one-period cash holdings. We assume that, for low income realizations of $\tilde{y}_{i 1}$, the insurance company pays an amount $x\left(\tilde{y}_{i 1}\right)-\pi(1+r)$ to the farmer - deducting the premium that is due from the payout. Obviously, this option is not available when income realizations are favorable, and the farmer has to pay the premium without receiving a payout.

Observe that the IOU prompts the farmer to make two choices. In period 0 , she has to decide whether to sign on to the IOU. And in period 1, she may decide whether to (strategically) default on the premium when the realization of $\tilde{y}_{i 1}$ is high and the farmer does not qualify for a payout. The farmer's utility in period 1 , as expected in period 0 , is now given by

$$
\beta\left[\bar{y}_{i}+\bar{x}_{i}-\frac{1}{2} \rho_{i}\left(\sigma_{y_{i}}^{2}+\sigma_{x}^{2}+2 \sigma_{x y_{i}}\right)-E\left[a_{i t-1} c\left(\tilde{z}_{i t}\right) \pi\right]\right]
$$

If condition (3.5) holds, it always pays the farmer to sign on to the IOU in period 0 and honor the agreement to pay the premium, compared to not signing on to the IOU. However, strategic default may be an even better solution for the farmer. To analyze this issue, we need to make assumptions about what happens when the farmer defaults in period 1 . Let $y^{*}$ be such that payouts have to be made when $\tilde{y}_{i 1}<y^{*}$ and no payouts are made when $\tilde{y}_{i 1} \geq y^{*}$. We assume that if the farmer refuses to pay $\pi(1+r)$ when $\tilde{y}_{i 1} \geq y^{*}$, she is punished for breach of

\footnotetext{
${ }^{15}$ If the farmer buys insurance in period $t-1$, then her cash flow in the next period, $\tilde{z}_{i t}$, has a lower spread than if the farmer had not bought insurance. With insurance, the farmer's cash-flow in period $t$ is $\tilde{z}_{i t} \in\left[\underline{z}_{i}, \bar{y}_{i}\right]$; without insurance, the farmer's cash-flow in period $t$ is $\tilde{z}_{i t} \in\left[\underline{y}_{i}, \bar{y}_{i}\right]$ and we know that $\underline{z}_{i}>\underline{y_{i}}$. Therefore, if she buys insurance in period 0 , then her ability to buy insurance in period 1 is enhanced compared to the situation where she did not buy insurance in period 0 . Hence, if she can buy insurance in period 0 , and the inter-temporal trade-off between period 0 and period 1 is such that she wants to buy insurance in period 0 , she will do so.
} 
agreement and the insurance company refuses to sell insurance to her in the future.

First we compare expected utility under the IOU and the standard insurance product (and going uninsured) for the no-default case. Let $W_{i}$ be the lifetime utility of the farmer in the presence of the IOU from period 2 onwards. If the farmer signs on to the IOU and commits to honoring the agreement, she expects to get:

$$
y_{0 i}+\beta\left[\bar{y}_{i}+\bar{x}_{i}-\frac{1}{2} \rho_{i}\left(\sigma_{y_{i}}^{2}+\sigma_{x_{i}}^{2}+2 \sigma_{y_{i} x_{i}}\right)-\pi(1+r)\right]+\beta^{2} W_{i}
$$

If she does not purchase IOU, even it if is available, she expects to get

$$
y_{0 i}+\beta\left[\bar{y}_{i}-\frac{1}{2} \rho_{i} \sigma_{y_{i}}^{2}\right]+\beta^{2} W_{i}
$$

Note that even if she does not buy an IOU in period 0 , we assume that the IOU remains available for the future periods, so that (maximum) life time utility from period 2 onwards also equals $W_{i}$. Comparing (3.7) to (3.8), the farmer will take the IOU in period 0 if the following condition holds:

$$
\bar{x}_{i}-\frac{1}{2} \rho_{i}\left(\sigma_{x_{i}}^{2}+2 \sigma_{y_{i} x_{i}}\right)-\pi(1+r) \geq 0
$$

Now we can compare the attractiveness of the standard insurance product and the IOU. Recall that, a farmer buys standard insurance if $\bar{x}_{i}-\frac{1}{2} \rho_{i}\left(\sigma_{x_{i}}^{2}+2 \sigma_{y_{i} x_{i}}\right) \geq\left(c\left(y_{0 i}\right) \pi / \beta\right)$. This condition may be combined with (3.9), yielding that the IOU (with no strategic default in period 1), is preferred to standard insurance if $\left(c\left(y_{0 i}\right) / \beta\right) \geq(1+r)$. It is natural to assume that $\beta \leq(1+r)$, i.e., the insurance company's opportunity cost of funds is not more than the rate of time discount for the farmer. Then, given $c() \geq$.1 , we have the immediate result that anyone buying standard insurance will also accept the IOU, while some who would not have bought standard insurance will take the IOU even when they can commit to not defaulting on their deferred payment of the premium.

Hypothesis 1: In the absence of opportunities for strategic default, an IOU is weakly preferred to a standard index insurance product. 


\subsubsection{Strategic defaults}

Strategic default can happen only if there is no payout from the insurance company and the farmer has to make the deferred premium payment. No payout from the insurance company happens with probability $F\left(y^{*}\right)$ and so a farmer will only consider strategic default with probability $F\left(y^{*}\right)$. Strategic default is the better option for the farmer when

$$
\begin{gathered}
y_{0 i}+\beta\left[\bar{y}_{i}+\bar{x}_{i}-\frac{1}{2} \rho_{i}\left(\sigma_{y_{i}}^{2}+\sigma_{x_{i}}^{2}+2 \sigma_{y_{i} x_{i}}\right)-F\left(y^{*}\right) \pi(1+r)\right]+\beta^{2} U_{i} \\
\geq y_{0 i}+\beta\left[\bar{y}_{i}+\bar{x}_{i}-\frac{1}{2} \rho_{i}\left(\sigma_{y_{i}}^{2}+\sigma_{x_{i}}^{2}+2 \sigma_{y_{i} x_{i}}\right)-\pi(1+r)\right]+\beta^{2} W_{i}
\end{gathered}
$$

where $U_{i}$ refers to the lifetime utility of the farmer if she defaulted in period 1 from period 2 onwards. The right-hand side is the payoff when the farmer does not default in period 1 and the left-hand side is when the farmer defaults. This condition boils down to the condition:

$$
\left[1-F\left(y^{*}\right)\right] \pi(1+r) \geq \beta\left(W_{i}-U_{i}\right)
$$

When a farmer signs on to the IOU with the intention to default if she can, the expected gain (from non-payment of premium) is given by $\left[1-F\left(y^{*}\right)\right] \pi(1+r)$. The expected loss in future periods (after the default) is given by the loss in life-time utility. Since the gain happens in one period and the loss kicks in from the next period onwards, the loss is discounted by that one period. This implies that impatient subjects are more likely to default.

Two important observations follow from this analysis, from which testable hypotheses can be gleaned. First, introducing the opportunity to strategically default in the next period weakly increases the returns to signing the IOU. By increasing the choice set, farmers cannot be made worse off and the proportion of farmers for whom signing up for the IOU is welfareincreasing goes up. However, not all farmers signing up for the IOU are legitimate clients the subset of farmers for whom condition (3.11) holds will not honor their contract in the future and default in the first period when they do not receive a payout.

Hypothesis 2: In the presence of opportunities for strategic default, uptake of an IOU will be greater than uptake of an IOU where strategic default is not possible. Uptake will also be greater than uptake of a standard insurance product.

Hypothesis 3: In the presence of opportunities for strategic default, this is a dominant strategy for farmers who are sufficiently impatient. Hence, some default will occur. 
It is also possible to formulate a corollary for these hypotheses. All measures taken by the insurance company to make strategic default more costly will lower uptake and default rates. These measures could include formal contracts and strict enforcement, but also leveraging social pressure from peers.

Corollary 1: Measures that reduce the benefits of strategic default will reduce uptake of the IOU, and also lower default rates.

\subsubsection{Trust}

We hypothesize that promoting index insurance via Iddirs improves trust in the insurance business. Peer pressure among Iddir members to repay the premium, together with the altruistic trust in Iddir structure, help to reduce strategic defaults. But how does trust can be captured in the farmer's considerations? We consider the example of trust for the standard index insurance. Farmers must be confident that the provider of insurance will pay up when the state of nature warrants a payout. Let this trust factor be represented by $p$, or the expected probability that the insurance company will pay when this is required. So far, we assumed $p=1$. Lack of trust, however, lowers the expected value of the payout. The expected value of payouts $\tilde{x}_{t}$ reduces to $p \bar{x}_{t}$ and its variance is $p^{2} \sigma_{x}^{2}$. If the farmer buys insurance in period 0 , the farmer's utility of the first two periods equals:

$$
y_{i 0}-c\left(y_{i 0}\right) \pi+\beta\left[\bar{y}_{i}+p \bar{x}_{i}-\frac{1}{2} \rho_{i}\left(\sigma_{y_{i}}^{2}+p^{2} \sigma_{x}^{2}+2 \sigma_{x y_{i}}\right)-E\left[a_{i 1} c\left(\tilde{z}_{i 1}\right) \pi\right]\right]
$$

As $p$ decreases, reflecting falling trust among farmers being served by the insurance company, fewer people are willing to buy the standard insurance. Similarly, a lack of trust also affects the uptake of IOUs. Suppose rainfall is low, then with probability $p$ the company is expected to payout $\bar{x}_{t}-\pi(1+r)$. Assume that farmers do not default. ${ }^{16}$ The farmer's utility in period 1 , as expected in period 0 , when adopting an IOU, is now given by:

$$
\beta\left[\bar{y}_{i}+p \bar{x}_{i}-\frac{1}{2} \rho_{i}\left(\sigma_{y_{i}}^{2}+p^{2} \sigma_{x}^{2}+2 \sigma_{x y_{i}}\right)-E\left[a_{i t-1} c\left(\tilde{z}_{i t}\right) \pi\right]\right]
$$

This leads us to hypothesis 4 as stated below.

\footnotetext{
${ }^{16}$ For simplicity we also assume that the insurance company comes to collect the deferred premium payment of the farmer even when it denies payment to farmers who are in a state with rainfall below the threshold. This facilitates the notation, but does not qualitatively affect the analysis.
} 
Hypothesis 4: An increase in trust will increase the uptake of standard index insurance as well as IOUs.

In addition to building trust in the insurance business, channeling through Iddirs is also potentially a useful mechanism to curb strategic default. Specifically, if all Iddir members honor their IOU contract and one farmer does not, there could be some 'social cost' from deviant behavior. Iddirs inform the insurance company about members who reneged on their contract. Then, the insurance company may not sell IOU to such farmers in the subsequent periods. In addition, the social cost can reinforce farmers not to default. Thus, in eq. (3.11), the LHS stays the same but the presence of social cost increases the RHS to $\beta\left(W_{i}-\underline{U}_{i}\right)+\delta$, where $\delta$ is the monetized value of the social cost. Corollary (1) applies, and leveraging the social dynamics and peer pressure among members of Iddirs should lower default rates.

\subsubsection{Basis risk}

Our experiment does not address basis risk explicitly. Yet, it is relevant to show how our model can be adjusted to deal with basis risk, because our experiment below is based on an index-based insurance product where payouts are triggered when the normalized difference vegetation index (NDVI) or greenery level falls below a threshold. While NDVI at the measurement point of a crop production zone (CPZ) is correlated with the NDVI for nearby farmers, there is only a statistical relationship between each CPZ's output and measured NDVI. In other words, if $\omega$ is the random NDVI variable, output $\tilde{y}_{i}(\omega)$ is a stochastic function of the type, $\tilde{y}_{i}(\omega)=G_{i}(\omega)+\varepsilon_{i}$, where $\varepsilon_{i}$ is random (See Section 2.2 for the details). In our analysis above, we used the correlation between $y$ and $x$, assuming that payouts are determined by yields through NDVI measures. With NDVI-based insurance, instead, $x$ is triggered by $\omega$ and not by $y$. This reduces the magnitude of the correlation (or covariance) between $x$ and $y$ but does not change the structure of our model. Basis risk reduces the value of $\beta\left[\bar{x}_{i}-\frac{1}{2} \rho_{i}\left(\sigma_{x_{i}}^{2}+2 \sigma_{y_{i} x_{i}}\right)\right]$ since the absolute value of $2 \sigma_{y_{i} x_{i}}$ is reduced. The probability of uptake goes down for higher levels of basis risk.

Does basis risk also affect strategic default? Consider condition (3.11) and realize that basis risk reduces the lifetime utility of farmers adopting IOU, $W$, from period 2 onwards. It follows immediately that the probability of strategic default increases, which may compromise the financial viability of the IOU. It is an empirical question whether this is a serious problem. 


\subsection{Intervention and randomization strategy}

We work together with Oromia Insurance Company (OIC) in Ethiopia. OIC, in collaboration with the Japan International Cooperation Agency (JICA), developed drought index insurance for crops in the Rift Valley zone of Ethiopia. The product was originally implemented in five districts: Boset, Bora, Ilfata, Adamitullu-Jido-Kombolcha (AJK), and Arsi Negele. It is marketed and sold twice per year, in months preceding the two rainy seasons (April and September). Insurance provides coverage against losses during the seedling and flowering stages of crop growth. The standard insurance is often marketed and sold via cooperatives. A household that buys insurance pays a premium of ETB 100 per policy (ETB $27.5=$ USD 1). To compensate for the delay in payment, the premium of the IOU was set at 106 , with the $6 \%$ surcharge for six months based on the $12 \%$ annual interbank rate in Ethiopia. The payout depends on the level of NDVI measured using satellite remote sensing. OIC applies a linearly proportional indemnification (LPI) approach in calculating the payouts. Farmers earn a maximum of ETB 667 per policy.

As in many other localities, take-up of the index insurance product is very low, approximately $7-8 \%$ of the population. OIC suspected two constraints are mainly responsible for low uptake: lack of liquidity and trust. To test this, and explore potential solutions, we designed an RCT with multiple treatment arms. Specifically, to relax a binding liquidity constraint we introduce an IOU and allow farmers to pay the premium after harvest. To make farmers trust the insurance system, we promoted the product through trained (randomly selected) Iddir leaders, who then train their members. During the training sessions, important aspects of agricultural insurance, the details of the insurance modality (either IOU or standard insurance) as well as the trustworthiness of insurance to mitigate the adverse welfare consequences of climate change induced covariate risks, were explained. Iddir leaders who participated on training were also informed to train and motivate their members on their regular Iddir meetings. Leaders from control Iddirs did not participate in the training, but they were informed through the conventional channel - through cooperative agents.

The study took place in three districts in the Rift Valley zone; Bora, Adami Tullu and Arsi Negele. This area regularly suffers from drought shocks. From each district, we randomly selected four kebeles, and 12 Iddirs per kebele, or a total of 144 Iddirs. We obtained lists of all Iddir members in our sample. On our pre-sales registration list, all households were 
registered as a member of only one Iddir. ${ }^{17} \mathrm{We}$ started the experiment with a baseline survey during April and May in 2016. During this period we also organized training sessions for Iddir leaders. Standard insurance and IOU were sold in May and June of 2016. We used multi-level randomization to assign the 144 Iddirs to six experimental arms. The treatment arms with the number of Iddirs and size of members presented in Table 3.1. ${ }^{18}$

Table 3.1: Randomization and treatment arms

\begin{tabular}{llcc}
\hline $\begin{array}{l}\text { Treatment } \\
\text { arms }\end{array}$ & Description of the treatment & $\begin{array}{c}\text { Iddir } \\
\text { size }\end{array}$ & $\mathrm{N}$ \\
\hline IBI $\left(\mathrm{T}_{1}\right)$ & Standard IBI through cooperative promotion & 16 & 853 \\
$\mathrm{IOU}\left(\mathrm{T}_{2}\right)$ & IOU through cooperative promotion without contract & 16 & 685 \\
IOUC $\left(\mathrm{T}_{3}\right)$ & IOU through cooperatives with binding contract & 16 & 633 \\
IBIM $\left(\mathrm{T}_{4}\right)$ & Standard IBI through Iddir promotion & 48 & 3056 \\
IOUM $\left(\mathrm{T}_{5}\right)$ & IOU through Iddir promotion without binding contract & 24 & 1887 \\
IOUMC $\left(\mathrm{T}_{6}\right)$ & IOU through Iddir promotion with binding contract & 24 & 1465 \\
\hline Total & & 144 & 8,579 \\
\hline Note: $\mathrm{IBI}\left(\mathrm{T}_{1}\right)=$ standard index-insurance marketed and sold via cooperatives; & IOU $\left(\mathrm{T}_{2}\right)=$ IOU
\end{tabular}

Note: IBI $\left(\mathrm{T}_{1}\right)=$ standard index-insurance marketed and sold via cooperatives; IOU $\left(\mathrm{T}_{2}\right)=$ IOU insurance without binding contract marketed and sold via cooperatives; IOUC $\left(\mathrm{T}_{3}\right)=$ IOU insurance with binding contract marketed and sold via cooperatives; IBIM $\left(\mathrm{T}_{4}\right)=$ standard index insurance marketed via Iddirs; IOUM $\left(\mathrm{T}_{5}\right)=$ IOU insurance without contract marketed via Iddirs; IOUMC $\left(\mathrm{T}_{6}\right)$ $=$ IOU insurance with binding contract marketed via Iddirs.

Iddirs assigned to IBI $\left(\mathrm{T}_{1}\right)$ are the control group. Members of these Iddirs were offered with the standard IBI promoted through the cooperatives, which is the statutory conventional (standard channel). Iddir members in IOU $\left(\mathrm{T}_{2}\right)$ were offered with the IOU product with promotion through cooperatives. Iddir members in IOUC $\left(\mathrm{T}_{3}\right)$ were also offered with IOU through cooperative promotions, but in addition, this group were required to sign a contract.

\footnotetext{
${ }^{17}$ A few households were members of 2 Iddirs, and to be considered in the experiment these households were asked to choose membership of only one Iddir.

${ }^{18}$ The number of households varies across the treatment arms because of variations in member size. We also purposefully over-sampled the groups in $T_{4}, T_{5}$ and $T_{6}$ to be able further sub-divided in follow-up work focusing on the Iddir channel. However, the number of subjects in the other treatment arms is sufficiently large for meaningful econometric analysis - exceeding 600 farmers per arm.
} 
This contract arrangement was designed to limit default ${ }^{19}$. Specifically, the contract stipulated that members were not only legally liable for the full premium, but in case of default, members will socially be responsible according to the rules and regulations of their Iddir. Farmers were also informed that a legal action could follow in case of contract breach. But the transaction costs associated with pursuing legal sanctions against defaulting clients outweigh the immediate benefits. Iddir members in IBIM $\left(\mathrm{T}_{4}\right)$ bought the standard insurance product with upfront payment promoted through Iddirs. Iddir members in IOUM $\left(\mathrm{T}_{5}\right)$ received the IOU with promotion via their Iddirs and, finally, Iddir members in IOUMC $\left(\mathrm{T}_{6}\right)$ received the most extensive package including delayed payment, via Iddir promotion, and with the contract. Iddirs assigned to the last three treatments, namely $\mathrm{T}_{4}, \mathrm{~T}_{5}$ and $\mathrm{T}_{6}$, are supposed to have a greater level of trust in insurance as their Iddir leader participated in a training and then trained their members about the benefits of insurance.

To verify whether randomization resulted in balanced groups, we estimate a series of OLS models, regressing household observables on treatment group dummies and a constant (see Tables 3.2a and 3.2b). Table 3.2a presents tests for socio-economic variables. It contains the following demographic characteristics of the household: Age (in years); Sex (male = 1; female $=0$ ); Marital status (married $=1$; not-married $=0$ ); Education (years of schooling); Family size; Total income in the last month (in ETB); Drought (a dummy taking the value of 1 if the household experienced a drought in the last three years); and Insurance (a dummy taking the value of 1 if the household had purchased index insurance during the past three years). The constant term reflects the mean of the comparison group (i.e., mean of IBI). The coefficients in Tables 3.2a and 3.2b indicate whether other groups are significantly different from the comparison group, and the Wald statistics test differences among groups.

\footnotetext{
${ }^{19}$ The contract states as follows: I, the undersigned individual, have signed a binding contract with Oromia Insurance Share Company in such a way that the Company provides me an index-based crop insurance policy of 100 ETB premium which entitles me a maximum payout of 667 ETB in case I incur crop losses due to drought during the $2015 / 16$ production year. In return, I will pay the premium of ETB 106 until October 30, 2016 upon harvesting my yield. If I fail to pay the indicated amount till the due date, I will be (1) legally liable for the amount of the promissory note (2) socially responsible according to the rules and regulations of my Iddir.
} 
Table 3.2a: Balance tests on socio-economic variables

\begin{tabular}{|c|c|c|c|c|c|c|c|c|}
\hline Variables & $\begin{array}{c}\text { Age } \\
\text { (years) }\end{array}$ & $\begin{array}{c}\text { Sex } \\
(1=\text { male })\end{array}$ & $\begin{array}{c}\text { Marital } \\
\text { status }\end{array}$ & $\begin{array}{c}\text { Education } \\
\text { (years) }\end{array}$ & $\begin{array}{c}\text { Family } \\
\text { size }\end{array}$ & Income & $\begin{array}{l}\text { Drought } \\
\text { dummy }\end{array}$ & $\begin{array}{c}\text { Bought } \\
\text { IBI } \\
\text { Before }\end{array}$ \\
\hline \multirow[t]{2}{*}{ IBIM } & -0.84 & 0.11 & -0.01 & 0.05 & 0.14 & -198.24 & 0.01 & -0.05 \\
\hline & (1.138) & $(0.089)$ & $(0.029)$ & $(0.426)$ & $(0.325)$ & (186.348) & $(0.049)$ & $(0.045)$ \\
\hline \multirow[t]{2}{*}{ IOUMC } & -1.10 & 0.16 & -0.02 & 0.05 & 0.43 & 62.84 & -0.06 & -0.03 \\
\hline & $(1.361)$ & $(0.096)$ & $(0.031)$ & $(0.473)$ & $(0.429)$ & $(248.140)$ & $(0.059)$ & $(0.051)$ \\
\hline \multirow[t]{2}{*}{ IOUM } & -0.48 & 0.12 & -0.02 & 0.34 & 0.32 & 303.50 & -0.05 & 0.08 \\
\hline & (1.356) & (0.109) & $(0.036)$ & $(0.559)$ & $(0.362)$ & $(558.396)$ & $(0.057)$ & $(0.064)$ \\
\hline \multirow[t]{2}{*}{ IOUC } & $-1.80 *$ & 0.02 & 0.00 & 0.11 & -0.38 & -160.66 & 0.05 & $-0.08 * *$ \\
\hline & $(0.781)$ & $(0.063)$ & $(0.022)$ & $(0.355)$ & $(0.244)$ & $(245.663)$ & $(0.028)$ & $(0.030)$ \\
\hline \multirow[t]{2}{*}{ IOU } & $-2.34 * *$ & 0.01 & -0.03 & 0.40 & -0.19 & -58.50 & $0.07 *$ & $-0.09 *$ \\
\hline & $(0.854)$ & $(0.072)$ & $(0.032)$ & $(0.396)$ & $(0.286)$ & $(400.776)$ & $(0.033)$ & $(0.036)$ \\
\hline Constant & $39.40 * *$ & $0.47 * *$ & $0.90 * *$ & $1.91 * *$ & $5.67 * *$ & $854.30 * *$ & $0.87 * *$ & $0.12 * *$ \\
\hline (mean of IBI) & $(0.901)$ & $(0.073)$ & $(0.025)$ & $(0.347)$ & $(0.299)$ & $(161.121)$ & $(0.041)$ & $(0.041)$ \\
\hline Test IBIM=IOUMC & 0.84 & 0.58 & 0.85 & 0.99 & 0.38 & 0.22 & 0.15 & 0.58 \\
\hline Test IBIM=IOUM & 0.76 & 0.94 & 0.76 & 0.55 & 0.46 & 0.36 & 0.18 & 0.01 \\
\hline Test IBIM=IOUC & 0.41 & 0.23 & 0.65 & 0.89 & 0.03 & 0.86 & 0.41 & 0.24 \\
\hline Test IBIM=IOU & 0.11 & 0.23 & 0.59 & 0.44 & 0.17 & 0.71 & 0.07 & 0.08 \\
\hline Test IOUMC $=$ IOUM & 0.66 & 0.71 & 0.88 & 0.59 & 0.76 & 0.67 & 0.87 & 0.05 \\
\hline Test IOUMC $=$ IOUC & 0.61 & 0.11 & 0.56 & 0.91 & 0.03 & 0.41 & 0.04 & 0.16 \\
\hline Test IOUMC $=$ IOUC & 0.30 & 0.11 & 0.71 & 0.49 & 0.10 & 0.78 & 0.005 & 0.08 \\
\hline Test IOUM=IOUC & 0.33 & 0.32 & 0.50 & 0.67 & 0.01 & 0.42 & 0.05 & 0.002 \\
\hline Test IOUM=IOU & 0.11 & 0.29 & 0.84 & 0.92 & 0.08 & 0.58 & 0.005 & 0.001 \\
\hline Test IOUC $=\mathrm{IOU}$ & 0.44 & 0.82 & 0.28 & 0.35 & 0.26 & 0.61 & 0.12 & 0.58 \\
\hline Observations & 8,579 & 8,579 & 8,579 & 8,579 & 8,579 & 8,579 & 8,579 & 8,579 \\
\hline
\end{tabular}

Notes: Robust standard errors in parentheses are clustered for 144 Iddirs; $* * * \mathrm{p}<0.01,{ }^{* *} \mathrm{p}<0.05,{ }^{*} \mathrm{p}<0.1$. Test gives p-values of Wald tests. The constant reflects the average uptake the control group (i.e., IBI).

Table $3.2 \mathrm{~b}$ presents similar balance tests for a vector of farming variables, capturing quantities of crops produced in the last cropping season (maize, haricot, teff, sorghum, wheat, and barely); a measure of total land under cultivation, and a dummy taking the value 1 if the household had any formal savings. Tables $3.2 \mathrm{a}$ and $3.2 \mathrm{~b}$ suggest that the randomization has worked well, especially regarding crop production at baseline-farmers of the different treatment groups produce on average the same crops. 
Table 3.2b: Balance tests for production variables and savings

\begin{tabular}{lllllllll}
\hline Variables & Maize & $\begin{array}{l}\text { Haricot } \\
\text { bean }\end{array}$ & Teff & Sorghum & Wheat & Barley & Land & Savings \\
\hline IBIM & 2.30 & 0.19 & -0.10 & 0.07 & 2.73 & -0.13 & -0.40 & 0.06 \\
& $(1.201)$ & $(0.158)$ & $(0.380)$ & $(0.144)$ & $(4.212)$ & $(0.132)$ & $(0.867)$ & $(0.068)$ \\
IOUMC & 2.23 & 0.17 & -0.34 & 0.01 & -1.03 & -0.14 & 0.82 & 0.01 \\
& $(1.513)$ & $(0.148)$ & $(0.375)$ & $(0.103)$ & $(1.767)$ & $(0.134)$ & $(1.178)$ & $(0.068)$ \\
IOUM & 0.54 & -0.01 & -0.05 & -0.01 & 0.74 & -0.20 & -1.24 & 0.02 \\
& $(1.167)$ & $(0.073)$ & $(0.452)$ & $(0.100)$ & $(2.112)$ & $(0.126)$ & $(0.850)$ & $(0.061)$ \\
IOUC & 0.40 & 0.03 & 0.10 & -0.08 & -0.85 & -0.06 & -0.05 & -0.03 \\
& $(0.730)$ & $(0.069)$ & $(0.295)$ & $(0.059)$ & $(0.991)$ & $(0.128)$ & $(0.415)$ & $(0.035)$ \\
IOU & 0.37 & -0.04 & -0.05 & -0.10 & -1.18 & -0.14 & 0.31 & 0.00 \\
Constant & $(0.751)$ & $(0.067)$ & $(0.406)$ & $(0.074)$ & $(1.277)$ & $(0.152)$ & $(0.617)$ & $(0.042)$ \\
(mean of IBI) & $6.54 * *$ & $0.21 * *$ & $1.35^{* *}$ & $0.19 *$ & $5.09 * *$ & $0.29 *$ & $8.06 * *$ & $0.21 * *$ \\
\hline Test IBIM=IOUMC & $(0.876)$ & $(0.061)$ & $(0.266)$ & $(0.081)$ & $(1.268)$ & $(0.121)$ & $(0.739)$ & $(0.044)$ \\
Test IBIM=IOUM & 0.12 & 0.18 & 0.92 & 0.59 & 0.65 & 0.27 & 0.18 & 0.49 \\
Test IBIM=IOUC & 0.12 & 0.30 & 0.66 & 0.27 & 0.39 & 0.58 & 0.70 & 0.16 \\
Test IBIM=IOU & 0.14 & 0.13 & 0.93 & 0.19 & 0.34 & 0.86 & 0.49 & 0.38 \\
Test IOUMC=IOUM & 0.25 & 0.22 & 0.52 & 0.84 & 0.40 & 0.47 & 0.04 & 0.92 \\
Test IOUMC=IOUC & 0.24 & 0.35 & 0.31 & 0.27 & 0.51 & 0.48 & 0.48 & 0.52 \\
Test IOUMC=IOU & 0.24 & 0.15 & 0.54 & 0.14 & 0.90 & 1.00 & 0.70 & 0.88 \\
Test IOUM=IOUC & 0.90 & 0.60 & 0.77 & 0.35 & 0.41 & 0.20 & 0.19 & 0.41 \\
Test IOUM=IOU & 0.88 & 0.61 & 1.00 & 0.19 & 0.31 & 0.57 & 0.14 & 0.78 \\
Test IOUC=IOU & 0.94 & 0.18 & 0.60 & 0.53 & 0.50 & 0.53 & 0.34 & 0.35 \\
\hline Observations & 8,579 & 8,579 & 8,579 & 8,579 & 8,579 & 8,579 & 8,579 & 8,579
\end{tabular}

Notes: Robust standard errors in parentheses, clustered for 144 Iddirs; $* * * \mathrm{p}<0.01, * * \mathrm{p}<0.05$, * $\mathrm{p}<0.1$. Test gives $\mathrm{p}$-values of Wald tests.

Compared to the control group IBI, the average ages in treatment groups IOU and IOUC, are slightly lower. Households in the IOU group experienced a bit more drought; and households in IOU and IOUC were slightly less likely to be insured before. The constant reflects the average in the control group. There are also some imbalances regarding family size (compare IBIM and IOUC as well as IOUM and IOUC), and regarding drought experiences. However, these differences are small, and we control for these observables in some regression models below. In the follow-up data collection we were able to retrieve all baseline respondents. 


\subsection{Results}

\subsubsection{Uptake}

We present post-treatment OLS regressions to analyze the determinants of uptake, and in particular examine how the different treatments affect uptake. Table 3.3 presents the results, and shows estimates with and without baseline controls. The results in column 1 of Table 3.3 are based on the subsample of treatment arms without promotion via Iddirs (i.e., IBI, IOU and IOUC). This enables us to understand the extent to which relaxing liquidity constraints conditional on promotion through the conventional cooperative channel enhances uptake. The constant in column 1 provides mean uptake of the standard insurance product, IBI. This uptake rate of $8 \%$ corresponds nicely with the pre-existing uptake figures by the insurance company for other kebeles (i.e., kebeles which were not considered in our study). An important result is that uptake can be tripled if farmers can delay payment until after the harvest season. In the IOU treatment, uptake increases by 17 percentage points without the contract. Liquidity therefore appears to be an important cause for low adoption rates. However, when farmers are offered an IOU product combined with a legal contract (IOUC), uptake drops to the level of the control group. The uptake-reducing effect of a binding contract suggests either that farmers are unsure about their ability to pay the premium in the future and fear repercussions in case of default, or that a subsample of farmers signing up for the normal IOU product intends to strategically default on their delayed payments in case the weather is favourable for production. This will be examined further below.

Column 2 in Table 3.3 reports on a similar model, but this time based on the subsample of groups exposed to insurance product promotion through Iddirs. The constant term under column 2 is $15 \%$, implying that uptake of the standard index product nearly doubles if Iddirs were used for promotion instead of cooperatives (compared to the constant under column 1). This suggests that trust is the underlying reason for increasing the uptake of weather index insurance. When promoted through Iddirs, the uptake of the IOU product increases by $28 \%$. Note that when the same IOU product is promoted through cooperatives, the increase in uptake was $17 \%$. This implies that an incredible increase in uptake in both the standard IBI and the innovative IOU is due to the use of Iddirs that hold higher levels of trust at the public. Table 3.3 also indicates that the existence of a contract has an attenuating effect on uptake. With a contract, the IOU product increases uptake by $17 \%$ as compared with the control group. Thus, uptake falls when contracts are used, compared to the increase in uptake of IOU without a contract which is $28 \%$ (See column 2). Introducing the contract for the 
innovative IOU with the cooperative promotion has no significant effect to increase uptake. The IOUC is significant in neither of the regressions while the IOUM and IOUMC variables are uniformly significant in all the regressions in Table 3.3.

Table 3.3: Uptake of insurance product

\begin{tabular}{|c|c|c|c|c|c|}
\hline Variables & $\begin{array}{c}\text { (1) } \\
\text { Restricted } \\
\text { sample: coop } \\
\text { promotion }\end{array}$ & $\begin{array}{c}(2) \\
\text { Restricted } \\
\text { sample: Iddir } \\
\text { promotion }\end{array}$ & $\begin{array}{c}\text { (3) } \\
\text { Full sample }\end{array}$ & $\begin{array}{c}\text { (4) } \\
\text { With } \\
\text { controls }\end{array}$ & $\begin{array}{c}\text { (5) } \\
\text { Adjusted } \\
\text { uptake }\end{array}$ \\
\hline $\mathrm{IOU}$ & $\begin{array}{l}0.17 * * \\
(0.067)\end{array}$ & & $\begin{array}{l}0.17 * * \\
(0.067)\end{array}$ & $\begin{array}{l}0.15^{* *} \\
(0.062)\end{array}$ & $\begin{array}{l}0.13 * * \\
(0.059)\end{array}$ \\
\hline IOUC & $\begin{array}{c}0.03 \\
(0.041)\end{array}$ & & $\begin{array}{c}0.03 \\
(0.041)\end{array}$ & $\begin{array}{c}0.03 \\
(0.041)\end{array}$ & $\begin{array}{c}0.02 \\
(0.037)\end{array}$ \\
\hline IBIM & & & $\begin{array}{c}0.07 \\
(0.058)\end{array}$ & $\begin{array}{c}0.05 \\
(0.053)\end{array}$ & $\begin{array}{c}0.07 \\
(0.058)\end{array}$ \\
\hline IOUM & & $\begin{array}{l}0.28 * * * \\
(0.102)\end{array}$ & $\begin{array}{c}0.35 * * * \\
(0.092)\end{array}$ & $\begin{array}{l}0.33^{* * *} \\
(0.086)\end{array}$ & $\begin{array}{l}0.31 * * * \\
(0.095)\end{array}$ \\
\hline IOUMC & & $\begin{array}{c}0.17^{*} \\
(0.097)\end{array}$ & $\begin{array}{l}0.25^{* * *} \\
(0.088)\end{array}$ & $\begin{array}{l}0.22 * * * \\
(0.069)\end{array}$ & $\begin{array}{l}0.23 * * \\
(0.089)\end{array}$ \\
\hline Age & & & & $\begin{array}{c}-0.00 * * * \\
(0.001)\end{array}$ & \\
\hline Sex & & & & $\begin{array}{l}-0.04 \\
(0.037)\end{array}$ & \\
\hline Marital status & & & & $\begin{array}{c}0.02 \\
(0.017)\end{array}$ & \\
\hline Education & & & & $\begin{array}{l}0.01 * * \\
(0.004)\end{array}$ & \\
\hline Family size & & & & $\begin{array}{l}0.02 * * * \\
(0.005)\end{array}$ & \\
\hline Income & & & & $\begin{array}{l}0.00 * * \\
(0.000)\end{array}$ & \\
\hline Drought experience & & & & $\begin{array}{l}-0.09 * * \\
(0.044)\end{array}$ & \\
\hline Bought IBI before & & & & $\begin{array}{c}-0.18 * * * \\
(0.055)\end{array}$ & \\
\hline Savings & & & & $\begin{array}{c}-0.14 \\
(0.046)^{* * *}\end{array}$ & \\
\hline $\begin{array}{l}\text { Indicators for crop } \\
\text { quantities }\end{array}$ & & & & Yes & \\
\hline Constant (IBI) & $\begin{array}{l}0.08 * * \\
(0.029)\end{array}$ & & $\begin{array}{l}0.08^{* * *} \\
(0.029)\end{array}$ & $\begin{array}{c}0.47 * * * \\
(0.116)\end{array}$ & $\begin{array}{l}0.08^{* * * *} \\
(0.029)\end{array}$ \\
\hline Constant2 (IBIM) & & $\begin{array}{l}0.15^{* * *} \\
(0.051)\end{array}$ & & & \\
\hline Observations & 2,171 & 6,408 & 8,579 & 8,579 & 8,579 \\
\hline Adjusted R-squared & 0.042 & 0.075 & 0.086 & 0.178 & 0.074 \\
\hline
\end{tabular}

Cluster robust standard errors in parentheses. ${ }^{* * *} \mathrm{p}<0.01,{ }^{* *} \mathrm{p}<0.05,{ }^{*} \mathrm{p}<0.1$. 
Column 3 essentially summarizes the results for the full sample. As before, we interpret the importance of trust, liquidity and contracts in increasing uptake. The result of the estimation for the constant term again reveals that the uptake of standard IBI is 0.08 , which is similar to the historical adoption statistics before this experiment. We now also learn that using Iddirs for the promotion of the standard insurance has no significant effect to increase uptake. While the coefficient for the IBIM variable equals 0.08 , it is not significantly different from zero. On the other hand, column 3 in Table 3.3 again indicates that the IOU promoted through cooperatives increases uptake by $17 \%$ compared to the standard insurance promoted through the same channel. Further, when the same product IOU is promoted through Iddirs without any contract, uptake increases by $35 \%$. This is the highest level of uptake when we compare all of the treatment arms. However, in cases where the IOU product is promoted through Iddirs, but members were required to sign a contract promising to repay the premium if the harvest is good, the increase in uptake is reduced to $25 \%$ (See column 3 in Table 3.3).

Similar results eventuate when we include our vectors of baseline controls - demographic variables and crop indicators. The following characteristics are associated with an increased propensity to purchase insurance: being younger and better educated, holding a larger farm, and having a higher income. Somewhat surprising, we also find that uptake is decreasing in prior experience with droughts and purchasing insurance. The latter correlation can suggest some farmers had already purchased some insurance elsewhere, or that these farmers have had bad experiences with index insurance in the past, for instance, because of basis risk. The former finding appears inconsistent with Cai and Song (2017) who simulate disaster experience in the lab, and find that gaining experience with shocks in a game promotes uptake of insurance in real life. In our case, it could imply that victims from (recent) droughts are more liquidity-constrained, and do not have the cash to pay the premium of the standard IBI product (leaving them more vulnerable to the adverse effects of future droughts - setting in motion a vicious circle of mutually enforcing poverty and vulnerability). It is interesting to observe the negative correlation between levels of own savings and the uptake of insurance. This could be because savings and insurance are substitute mechanisms to hedge against shocks. In line with this, Binswanger (2012) argued that index insurance can be less valuable for farmers with access to alternative approaches to cope with shocks. Finally, column 5 in Table 3.3 presents default adjusted uptake for robustness checks, but detail interpretations about this were given in Section 3.5 under robustness analysis. In general, as far as adoption is concerned, the experiment reported in this chapter reveals two important results on uptake 
of weather index insurance. First, if farmers can delay insurance payment towards shortly after harvest (IOU), the uptake of weather insurance increases almost threefold. Second, promoting insurance through socially-trusted customary institutions increases uptake of the IOU, but not of the standard insurance product. Neither do an IOU promoted through the conventional channel is promising in the prevalence of defaults. Sustainable adoption of weather insurances thus requires innovative promotion of the novel IOU product through trusted customary institutions.

\subsubsection{Defaults}

The success of the IOU intervention and its potential for scaling up depends on the combined effect of uptake and defaults. Enhanced uptake can be positive, but an increase in default limits the scope for scaling up. Hence, can we attribute the increase in uptake under IOU promoted through Iddirs to the relaxation of the liquidity constraint and improvement in trust? As discussed in Section 2, the prospect of strategic default may also tempt some farmers to adopt the IOU product. Exploring this issue in detail requires observing uptake and defaults of IOU over several years. This would enable identification of the sub-group of farmers who gamble on bad weather payout, but would never repay otherwise. Unfortunately we only observe defaults for one insurance period. Nevertheless, we are able to analyze defaults and identify approaches to mitigate defaults. Table 3.4 shows average default rates across the six experimental arms.

Table 3.4: Average default rates

\begin{tabular}{lc}
\hline Variables & Defaults \\
\hline IOU $\left(\mathrm{T}_{2}\right)$ & $0.17(0.37)$ \\
$\operatorname{IOUC}\left(\mathrm{T}_{3}\right)$ & $0.14(0.35)$ \\
$\operatorname{IOUM}\left(\mathrm{T}_{5}\right)$ & $0.09(0.25)$ \\
$\operatorname{IOUMC}\left(\mathrm{T}_{6}\right)$ & $0.05(0.23)$ \\
\hline Observations & 1,514
\end{tabular}

Note: Standard deviations are in parenthesis. Defaults prevail only for IOU products; the standard insurance requires upfront premium payment, and does not involve any postponed due, or defaults on repayment

For each IOU treatment arm, we consider the sub-group of farmers taking up insurance, and next calculate the percentage of these farmers that reneged on (delayed) payment of the premium. Observe there can be no default for the standard index insurance product, because payment of this premium has to be up-front. The standard IOU product has a default rate of 
nearly $17 \%$, which may compromise financial viability of such a product. Assuming an actuarially fair insurance product, this implies that the IOU premium has to increase to accommodate default. For a default rate of $17 \%$, the premium has to increase by more than $20 \%(1 / 0.83)$. If we also compensate for the opportunity cost of time $(6 \%)$, then the total price of IOU policies will have to be $26 \%$ higher than the price of regular IBI policies. But whether such pricing will curtail demand for the insurance product is questionable.

However, Table 3.4 suggests that it may be possible to reduce default rates associated with delayed payment. While uptake in the IOUC treatment was low (not exceeding the control group, the contract hardly brings down default (see Table 3.4). The default rate in the IOUC treatment is $14 \%$, nearly as high as in the basic IOU arm. Promotion of the IOU product via Iddirs, however, matters greatly for default. In the IOUM treatment, the default rate falls to $9 \%$ - a reduction of nearly $50 \%$ compared to the basic IOU. Adding the legal contract further pushes down default rate to $5 \%$. Compensating for the opportunity cost of time and the additional risk of default therefore implies the actuarially fair premium of the IOUMC product has to be $11 \%$ higher than that of the basic index insurance product.

It is reasonable to assume that the $5 \%$ of the defaulting clients in the IOUMC arm are farmers who cannot afford to pay the premium. These farmers risk judicial consequences, and may face social sanctions from Iddirs. It can also be the case that a small fraction of the clients who adopted insurance would be unable to pay. This can happen due to the challenges of basis risk and liquidity constraints. In contrast, farmers defaulting in the basic IOU arm may be either unable or unwilling to pay. Random assignment of Iddirs to treatment arms implies we have no reason to assume that (location-specific) basis risk is correlated with intervention status. Hence, farmers in the IOU arm are, on average, not less able to pay the premium than farmers in the IOUMC arm. If 5\% of these farmers were unable to pay the premium (e.g., because of basis risk), then, $12 \%$ of these farmers has strategically defaulted on payment of the premium. To probe whether many farmers are induced to take up the IOU by the prospect of strategic defaulting, we can compare take-up rates for arms where defaulting is costly.

We offered IOU with a contract through both cooperatives and Iddirs $\left(\mathrm{T}_{3}\right.$ and $\left.\mathrm{T}_{6}\right)$. Farmers committed to repay the future premium should be unaffected by this contract, but the expected cost of default increases. This should reduce uptake for strategic defaulters (and for farmers who are uncertain about their future ability to pay). Table 3.5 compares uptake rates for the different IOU treatments, with and without contract, to uptake rates of standard insurance. 
Table 3.5: Uptake with and without contract

\begin{tabular}{|c|c|c|}
\hline Variables & $\begin{array}{c}\text { (1) } \\
\text { Parsimonious }\end{array}$ & $\begin{array}{c}\text { (2) } \\
\text { With controls }\end{array}$ \\
\hline \multirow[t]{2}{*}{$\mathrm{IOU}\left(\mathrm{T}_{2}\right)$} & $0.17 * *$ & $0.14 * *$ \\
\hline & $(0.065)$ & $(0.060)$ \\
\hline \multirow[t]{2}{*}{$\operatorname{IOUC}\left(\mathrm{T}_{3}\right)$} & 0.03 & 0.02 \\
\hline & $(0.041)$ & $(0.044)$ \\
\hline \multirow[t]{2}{*}{$\operatorname{IOUM}\left(\mathrm{T}_{5}\right)$} & $0.35 * * *$ & $0.33 * * *$ \\
\hline & $(0.094)$ & $(0.066)$ \\
\hline \multirow[t]{2}{*}{$\operatorname{IOUMC}\left(\mathrm{T}_{6}\right)$} & $0.25 * * *$ & $0.16 * * *$ \\
\hline & $(0.086)$ & $(0.052)$ \\
\hline Kebele f.e. & No & Yes \\
\hline Additional controls & No & Yes \\
\hline \multirow[t]{2}{*}{ Constant } & $0.08 * *$ & -0.03 \\
\hline & $(0.029)$ & $(0.143)$ \\
\hline $\mathrm{F}$ test $(I O U>I O U C)$ & $7.90 * * *$ & $8.29 * * *$ \\
\hline $\mathrm{F}$ test $(I O U M>I O U M C)$ & 0.73 & $6.43 * *$ \\
\hline Observations & 5,523 & 5,523 \\
\hline Adjusted R-squared & 0.085 & 0.346 \\
\hline
\end{tabular}

Robust standard errors in parentheses are clustered at the Iddir level. Kebele fixed effects capture 12 kebeles across 3 Districts. Additional controls include Age, Male, Married, Education level, Family size, Income in last month, Drought dummy, Insured before dummy, Maize production, Haricot bean production, Teff production, Sorghum production, Wheat production, Barley production, Land size, and Savings. ${ }^{* * *} \mathrm{p}<0.01,{ }^{* *} \mathrm{p}<0.05, * \mathrm{p}<0.1$.

While the IOU promoted through cooperatives but without contract increases uptake, we find no significant difference between standard insurance and the IOU augmented with a contract. Since both IOU and IOUC were promoted through cooperatives, this difference cannot be attributed to the promotion channel. Rather, the results suggests that nearly all of the additional adoption induced by delayed payment is either motivated by the prospect of strategic default, or the result of farmers who are unsure about their ability to pay the future premium. This is hardly a promising outcome for the insurance company.

However, there is actually a scope to increase sustainable uptake of the IOU, when promotion through Iddirs is leveraged to build farmers' trust in insurance. This is evident from the uptake of IOUM and IOUMC in Table 3.5. While, as before, introducing the contract lowers uptake (uptake of IOUMC < uptake of IOUM), we now find that the adoption rate of the IOU 
with contract remains significantly higher than that of the standard insurance product $(I O U M C>I B I)$. Hence, trust in product that might have been built from promotion through Iddirs matters in increasing uptake. Overall, the uptake of IOU with contracts promoted through cooperatives is not significantly different from the uptake of the standard insurance. However, if promotion through Iddirs that builds trust can be leveraged, uptake of the IOU with a contract remains significantly higher than the uptake of standard insurance (though it is significantly lower than the IOU promoted by Iddirs but without a contract).

To capitalize on the promise of the IOU promoted through Iddirs with contract enforcement for sustainable scale up, let us look at defaults in more detail. Figure 1 shows default rates for the four IOU interventions (i.e. $\mathrm{T}_{2}, \mathrm{~T}_{3}, \mathrm{~T}_{5}$ and $\mathrm{T}_{6}$ ).

Figure 1: Default rates across IOU treatments

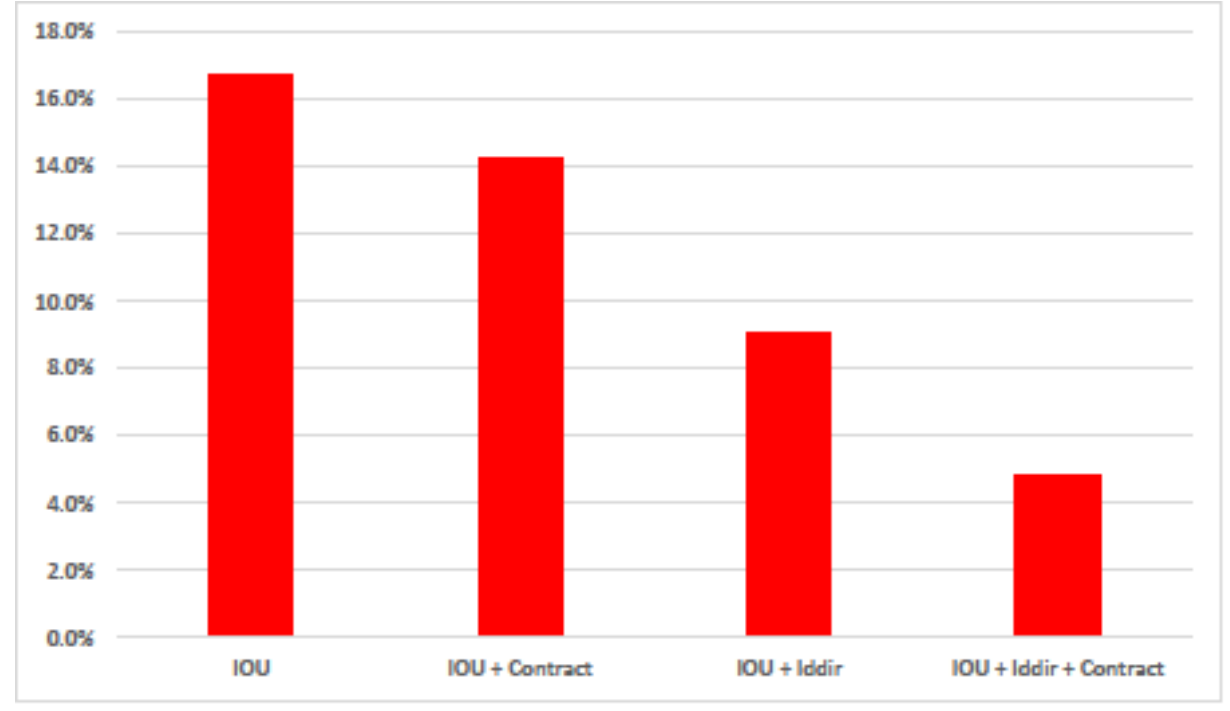

As explained before the default rate is highest for the IOU promoted through cooperatives without contract, and the default rate is the lowest for the IOU promoted through Iddirs with a contract. The lowest default rate in the latter case can be reasonably attributed to trust and peer pressure.

Combining the point estimates in Table 3.5 and Figure 1 enables us to define an 'upper bound' on the share of strategic defaulters in the population. Introducing the contract lowers the share of farmers adopting the IOU by 12 percentage points (from $14 \%$ to $2 \%$ ). Among those who adopt the IOU, some $14 \%$ can default the next season by the same trend. Hence, at most, $26 \%$ of the population can be suspected to be strategic defaulters. A similar estimate follows from considering the IOU promoted by Iddirs. The share of adopters drops by $17 \%$ 
(from $33 \%$ to $16 \%$ ), and some $5 \%$ of the adopters can default the next season. The maximum share of strategic defaulters in the population is therefore $22 \%$. As mentioned, our estimate of $22-26 \%$ should be interpreted as an upper bound default rate. This is due to the fact that in addition to genuine strategic defaulters, this group also includes, farmers who fail to adopt due to fear of inability to repay the deferred premium.

Table 3.6: Default rates in IOU promoted via Iddirs

\begin{tabular}{lccc}
\hline Variables & $(1)$ & $(2)$ & $(3)$ \\
& Parsimonious & Additional controls & Heckman \\
\hline Iddir & -0.08 & -0.04 & -0.04 \\
Contract & $(0.175)$ & $(0.049)$ & $(0.049)$ \\
& -0.02 & -0.05 & $-0.05^{*}$ \\
Iddir $\times$ Contract & $(0.040)$ & $(0.028)$ & $(0.028)$ \\
& -0.02 & 0.03 & 0.03 \\
Clustered standard errors & $(0.105)$ & $(0.042)$ & $(0.042)$ \\
Additional Controls & Yes & Yes & Yes \\
Constant & No & Yes & Yes \\
& 0.17 & 0.01 & 0.04 \\
Inverse Mills Ratio & $(0.151)$ & $(0.047)$ & $(0.065)$ \\
Observations & 1,514 & & $-0.55^{* * *}$ \\
Adjusted R-squared & 0.015 & 1,514 & 4,670 \\
\hline
\end{tabular}

Note: Robust standard errors in parentheses are clustered at the Iddir level in (1) and (2). In (3) we use all additional controls in the selection model. We estimate the Inverse Mills Ratio separately using a two-step method. Additional controls include Age, Male, Married, Education level, Family size, Income last month, Drought dummy, Insured before dummy, Maize production, Haricot production, Teff production, Sorghum production, Wheat production, Barley production, Land size, and Savings. $* * * \mathrm{p}<0.01, * * \mathrm{p}<0.05,{ }^{*} \mathrm{p}<0.1$.

Table 3.6 analyzes, in more detail, the effect of Iddir promotion, contracts, and a combination of promotion and contract on default rates. Surprisingly, it appears as if there is hardly a significant correlation between treatment status and default rates. This is, however, consistent with data summarized in Figure 1. While differences in mean default rates across treatment arms are considerable, they are not significant when controlling for Iddir fixed effects. What can explain this finding? As the underlying causes for this, in Section 3.4.3 below, we analyze how basis risk ultimately induces extreme clustering of defaults among few Iddirs. 


\subsubsection{Extreme clustering of defaults among few Iddirs}

Closer inspection of our data reveals extreme clustering of defaults in a small number of Iddirs - reducing the statistical power of our analysis. More specifically, all 134 defaults in our dataset come from only 4 Iddirs out of the subsample of 90 Iddirs that were offered IOUs. In each of these Iddirs, every member who had purchased an IOU policy defaulted. We conducted 12 focus group discussions (FGD) at Iddir level. Participants strongly argued that members of defaulting Iddirs in our sample were severely suffered from drought but did not qualify for payout. But OIC justifies that smallholders were not entitled to earn payout since the threshold values for NDVI were (just) reached. There insured farmers 'complained that they have faced severe yield losses due to drought (FDG discussions report). This is the wellknown problem of basis risk that according to our theoretical model induces default ${ }^{20}$. As a consequence of basis risk, Iddir members appear to have signaled their collective discontent by coordinating on an outcome of complete default. We did not anticipate such coordinated responses, and believe that this finding points to an important potential risk for parties offering services with delayed payment. Coordinated responses of smallholders might complicate re-insurance on international markets, and threaten the liquidity of insurance companies and the viability of IOU products.

\subsection{Robustness analysis}

First, to understand the sensitivity of uptake under the prevailing defaults, we consider how different treatments affect adjusted uptake, defined as, $U^{\prime}=U(1-D)$, whereas $U^{\prime}, U$ and $D$ represent adjusted uptake, uptake and defaults rates, respectively. The results for adjusted uptake as an alternative outcome indicator are presented under column 5 in Table 3.3. The results are consistent with results for unadjusted uptake variable. That is the combination of the marketing intervention with an IOU outperforms (See column 5 in Table 3.3).

Second, we found that marketing via Iddirs promotes uptake of IOU insurance. We also hypothesized that the mechanism linking Iddirs to uptake is enhanced trust. However, some literatures identify additional reasons why group-based marketing might boost adoption. Specifically, understanding insurance (knowledge) is identified as an important determinant as trust and liquidity constraints in adopting weather insurance (Cole et al. 2012). However,

\footnotetext{
${ }^{20}$ Still, we lack some quantitative data to further assess whether farmer-level drought intensities were sufficiently severe to warrant payouts.
} 
our initial intervention does not enable us to cleanly distinguish the effect of information (knowledge), from the effects of liquidity and trust as mechanisms for increase in uptake. In this experiment both an existing index insurance product and the IOU were sold with promotions via both cooperatives and Iddirs. The products are offered by the same insurance company. Based on this, one might argue that the differential increase in uptake among the insurance products promoted via Iddirs is due to the differential effect in information (knowledge) across contexts, in terms of higher financial literacy, or higher cognitive ability.

To test this hypothesis and be able to untangle whether knowledge differentials prevail among the treatment groups and matter in increasing uptake, we organized a midline survey after the experiment. We collected data on seven cognitive ability questions, four financial literacy questions and 5 questions about index insurance. We constructed 3 indices by summing the correct answers per category: a cognitive ability index; a financial literacy index; and an index on understanding the logics of weather index insurance. Next, we regress these three knowledge indices on a vector of treatment dummies. Results were presented in Table 3.7.

Table 3.7: Financial literacy
(1)
(2)
(3)

\begin{tabular}{lccc} 
Variables & Cognitive Ability & Financial Literacy & IBI Understanding \\
\hline IBIM & 0.03 & -0.11 & -0.12 \\
IOUMC & $(0.239)$ & $(0.183)$ & $(0.167)$ \\
& 0.01 & -0.02 & -0.31 \\
IOUM & $(0.262)$ & $(0.189)$ & $(0.206)$ \\
& 0.35 & 0.04 & 0.12 \\
IOUC & $(0.225)$ & $(0.197)$ & $(0.202)$ \\
& 0.27 & 0.04 & -0.15 \\
IOU & $(0.166)$ & $(0.125)$ & $(0.114)$ \\
& 0.20 & 0.07 & -0.05 \\
Constant & $(0.170)$ & $(0.141)$ & $(0.144)$ \\
(mean of IBI) & 4.29 & 1.97 & 3.70 \\
\hline Observations & $(0.173)^{* * *}$ & $(0.144)^{* * *}$ & $(0.128)^{* * *}$ \\
Adjusted R-squared & 8,579 & 8,579 & 8,579 \\
\hline
\end{tabular}

Cluster robust standard errors in parentheses are adjusted for 144 clusters, ${ }^{* * *} \mathrm{p}<0.01,{ }^{* *} \mathrm{p}<0.05, *$ $\mathrm{p}<0.1$. 
As Table 3.7 indicates, none of the groups scored differently on any of the indices, suggesting that differences in information (knowledge) do not explain variation in uptake across the treatment arms. Further, observe from the constants of the three models that the analysis does not suffer from 'floor' or 'ceiling' effects - there is no bunching of respondents towards the end of the lower or upper tail. Hence, the fact that knowledge differences are insignificant across the treatment arms, affirm that mere information sharing is not an effective mechanism for the increase in uptake, rather it is the in-built altruistic trust in Iddir setups that increases the uptake of insurance.

\subsection{Conclusion}

Uptake of agricultural insurance among African smallholders remains very low. This not only impairs the ability of smallholders to smooth consumption levels over time, it also mitigates incentives for intensification and modernization. Finding (scalable) approaches to boost uptake is an important topic on the international research agenda. The main finding of our experiment in this chapter is that uptake of weather insurance can be enhanced by promoting innovative IOU products through trusted customary institutions. Novel insurance products featuring delayed payment of premiums are useful to shift expenditures from periods when capital is very scarce (and the marginal utility of money is very high) to periods with relatively abundant access to money. Promoting such products through socially trusted customary institutions like Iddirs can be successful in enhancing uptake. However, to make this a cost-effective scalable intervention, it is important to ensure that default rates on delayed payments are low. While delayed payment does not matter in a world inhabited solely by Homo economicus (who would save surpluses from capital-abundant periods to finance worthwhile investments in capital-scarce periods), it is well-known that behavioral factors and market imperfections may restrict the pursuit of such strategies in practice (Levitt and List 2008; Duflo et al. 2011; Baland et al. 2011). Our pilot suggests that default rates of a simple IOU product are relatively high (17\%), perhaps curbing the enthusiasm of insurance companies to further scale up products involving delayed payment. However, promoting IOUs through Iddirs with binding contracts reduces the defaults to only 5\%. Further, we also find that defaults are concentrated in a very small number of Iddirs. This suggests that members of these few Iddirs were coordinated on default since they expected but did not earn payouts as a consequence of basis risk. 
Co-ordination on non-payment - invited by basis risk or otherwise - threatens the viability of the IOU product. From the group-lending literature it is well-known that a bank's possibility to harness 'social capital' may, under certain conditions, be undermined by borrowers who collectively collude against the bank (Besley and Coate 1995; Ghatak 1999). Our IOU intervention seems to some extent be affected by a similar problem. Further research should explore the potential of combining IOU insurance with joint liability contracts with dynamic incentives at the Iddir level. This could further reduce the prevalence of (strategic) defaults through the mechanisms like peer pressure, monitoring the behavior of current adopters and enforcing repayment. In addition, future research could focus on identifying other innovative approaches to address the liquidity problem that appears to cripple the insurance market, without exposing organizations involved in selling insurance to undue risks. 


\title{
Chapter 4
}

\section{Effects of index insurance on demand and supply of credit: Evidence from Ethiopia}

\begin{abstract}
Index-based insurance is increasingly recognized as a climate risk management strategy that can benefit the poor. This paper focuses on the question whether the adoption of index insurance improves access to financial markets and reduces credit rationing. Our empirical analyses focus on Ethiopia and leverages the situation in which index insurance is not yet available in some areas. With an innovative identification strategy we control for potential selection biases by forecasting potential insurance adopters, combined with a cross-sectional double-difference method. As credit rationing can take the form of either supply-side quantity rationing, in which case potential borrowers who need credit are involuntarily excluded from the credit market, or demand-side rationing, such that borrowers self-select and voluntarily withdraw from the credit market to avoid transaction costs and threats to their collateral, we differentiate supply-side and demand-side forms. Employing a direct elicitation method to determine credit rationing status, we determine that $38 \%$ of the sample households are credit constrained. Our analyses suggest that index insurance reduces supply-side credit rationing but not demand-side rationing.
\end{abstract}

Keywords: Index insurance; Credit rationing; Ethiopia; Smallholders

This chapter is based on:

Belissa, Lensink and Winkel (2018): Effects of Index Insurance on Demand and Supply of Credit: Evidence from Ethiopia. American Journal of Agricultural Economics revised and submitted. 


\subsection{Introduction}

As innovative hedging instruments, index-based insurances (IBIs) can mitigate risks of drought and seasonality-based weather variations resulting from climate change. An attractive feature of this innovation is that insurance payout occurs when an objective index falls below a given threshold. The index is usually based on a measure of the intensity of rainfall or direct measures of the yields for the specific geographic zone that the insurance contract covers (Carter et al. 2014). Ideally, an index is closely correlated with insured losses, objectively quantifiable, and publicly verifiable, so that it cannot be manipulated by either the insurer or the insured (Barnett et al. 2008; Zant, 2008). While the uptake of IBIs remains unexpectedly low $^{21}$ (Cole et al. 2012; Dercon et al. 2014; Hill et al. 2013; Takahashi et al. 2016) and demand for IBI is generally sluggish (Carter et al. 2014), several studies point to potentially substantial wealth effects of IBI (Elabed \& Carter 2014; Janzen \& Carter 2013; Karlan et al. 2014). The adoption of IBI can induce households to make more prudential investments, and better manage consumption risk (through stabilizing savings or asset accumulation), which could protect those households from sliding into poverty traps (Barnett et al. 2008).

IBI delinks loss assessments from individual behaviour, which means it can avoid moral hazard and adverse selection problems (Barnett et al. 2008; Skees 2008), and hence may improve credit access. However, there is almost no empirical evidence on the effect of the adoption of IBI on demand and supply of credit. The study included in this chapter is among the first to explicitly study the impact of index-based insurance on credit rationing by using data from a drought-prone area in the Rift Valley zone of Ethiopia. Although little empirical evidence details the effect of IBI on credit rationing, some earlier studies provide insights. In a framed field experiment in China, Cheng (2014) studies the effect of offering IBI to households that voluntarily withdraw from the credit market. All subjects participate in both the control and treatment game. The results demonstrate that more than half of the farmers decide to apply for credit when IBI is made available to them. Roughly two-thirds of credit diverters also choose to use their loan for productive investments rather than consumption

\footnotetext{
${ }^{21}$ A potentially important reason for the low uptake of IBI is the so-called basis risk, which arises due to the imperfect correlation between computed indices and actual losses (Cummins et al. 2004; Jensen et al. 2014). Especially in developing countries, where terrestrial weather stations are sparse, the discrepancy between losses indicated by the index and actual losses realized at the farm level is high (Clarke et al. 2012).
} 
when IBI is available. This behaviour can be explained in multiple ways: IBI reduces production risk, but the risk associated with using the loan for consumption remains constant or might even increase.

Further insights come from Giné and Yang (2009). They randomly offered maize farmers in Malawi either a loan or a loan plus insurance, which indemnifies them if rainfall is insufficient. The insurance could be bought at an actuarially fair premium. Although they expected that farmers would prefer the insured loan, demand for the basic loan was $13 \%$ higher than that for the insured loan. To explain why insurance did not lead to higher demand for credit, the authors suggest that the limited liability clause of the basic loan provided some implicit form of insurance. It should be noted, though, that in contrast to our study, Giné and Yang (2009) do no focus on the impact of a stand-alone insurance product. As Carter et al. (2016) suggest, a stand-alone insurance product does not provide additional benefits to farmers who have low collateral. If no formal insurance is available, only farmers with high collateral may choose not to borrow, because they do not want to put their collateral at risk.

Some studies focus on the impact of IBI on credit supply. On the one hand, some studies suggest that IBI is likely to relax supply-side constraints and quantity rationing as lenders tend to lower interest rates and lend more to insured clients as it may reduce default risk (Giné \& Yang 2009; Mahul \& Skees 2007; McIntosh et al. 2013). Other studies, however, suggest that insurance could decrease demand and supply of credit. As IBI contracts are sometimes unable to trigger payout, even when the insured incurs significant yield losses due to weather risk, the inability to repay the loan and hence loan defaults due to cash outflows for the premium may increase. Alternatively, a stand-alone insurance product might increase the minimum welfare level to which defaulting households can be pushed back in case they are caught. In this situation, incentives to repay are reduced as the welfare costs of defaulting are lower. Lenders who take loan default potentials into account could then limit credit supply in areas where IBI is implemented (Banerjee 2000; Clarke \& Dercon 2009; Farrin \& Miranda 2015).

Our study is unique in that it provides empirical evidence on the impact of IBI on demandside (i.e. risk and transaction cost rationing) and supply-side credit rationing. The analyses suggest that IBI reduces supply-side rationing (quantity rationing) but not demand-side rationing. The impact of IBI uptake on reducing supply-side credit rationing seems to be relatively large as the relative probability of being non-credit rationed rather than being supply-side credit rationed for IBI holders is more than double the corresponding relative 
probability for non-IBI holders with the same characteristics. In the next section, we explain the method we use to determine credit rationing. After detailing the study setting, the working of IBI, the sampling procedure, and identification strategy, we present the estimation results and conclude with some relevant insights.

\subsection{Credit rationing}

If rural financial markets were perfectly competitive, with symmetric information and costless enforcement, lenders could arrange conditional credit contracts according to borrowers' behaviours. However, rural financial markets in developing countries often feature information asymmetries, leading to moral hazard and adverse selection problems, as well as higher transaction costs associated with loan monitoring and contract enforcement (Besanko \& Thakor 1987; Bester 1985; Boucher et al. 2008; Fafchamps et al. 1997; Jaffee \& Russell 1976; Lensink \& Sterken 2002; Stiglitz \& Weiss 1981; Vandell 1984; Williamson 1986). These classic incentive problems make conditional credit contracts restrictive and infeasible (Deaton 1992; Dowd 1992; Bernheim and Whinston 1998; Ghosh et al. 2000; Lensink and Sterken 2001; Kumar and Matsusaka 2009).

Rural financial markets typically are characterized by credit rationing, such that lenders set a ceiling and avoid extending additional credit even if borrowers are willing to pay higher rates of interest. If firms are credit constrained, an increase in access to credit will facilitate investment. The ability to borrow also constitutes a major source of private insurance against consumption fluctuations that arise from idiosyncratic shocks in rural areas though, so credit rationing can have far reaching and adverse welfare consequences (Chetty and Szeidl 2007). These include decreased consumption smoothing (Morduch 1995; Zimmerman \& Carter 2003) and limited investment in high-risk high-return inputs or applications of chemical fertilizer (Beegle et al. 2003; Guarcello et al. 2010; Hill et al. 2013; McIntosh et al. 2013). Credit constrained households might be forced to lower their expenditures on nutrition, which often leads to persistent health outcomes, such as stunted child development (Islam \& Maitra 2012). Limited access to credit, which serves as a tool to smooth household income fluctuations, can also prompt school dropouts, due to the need for increased child labour on landlord farms. This in turn lowers the level of human capital development (Beegle et al. 2003; Guarcello et al. 2010). Exposure to repeated downturns and inaccessible credit thus reinforces vulnerability and perpetuates poverty by shaping both the behaviours and the outcomes of the poor (Fafchamps 2010). 
Credit rationing can take the form of either supply-side quantity rationing, in which case potential borrowers who need credit are involuntarily excluded from the credit market, even though they would be willing to pay higher interest rates, or demand-side rationing, such that borrowers self-select and voluntarily withdraw from the credit market to avoid transaction costs and threats to their collateral (Bester 1987; Boucher et al. 2008). Demand-side credit rationing thus includes both risk and transaction cost rationing. Risk rationing is prevalent in the absence of insurance markets when lenders shift contractual risks to borrowers; borrowers respond by voluntarily withdrawing from the market, even though the value of their collateral is sufficient to qualify them for a loan contract (Boucher et al. 2005; Boucher \& Guirkinger 2007). Similarly, transaction cost rationing arises when the costs of waiting for, processing, and administering the loan are too high for borrowers (Boucher et al. 2009).

Noting the several definitions of credit rationing, this study uses a direct elicitation method (DEM) (Boucher et al. 2009) to identify the credit rationing status of each household. With this method, we can identify credit constrained households according to their decision to borrow and the lender's decision to supply credit. The credit rationing module that we adopted (see Boucher et al. 2009 for the details) starts by asking whether the respondent has applied for a formal loan in the past five years. If so, it asks whether the application has been accepted. Households that have not applied for a formal loan indicate their reasons for not applying. According to their responses, all households can be categorized into one of four mutually exclusive groups: credit unconstrained, quantity (or supply-side) rationed, risk rationed, and transaction cost rationed. Households that apply for formal loans and receive them are categorized as unconstrained. However, if households applied for (more) credit at the prevailing interest rate and their application was rejected, they are classified as quantity rationed. If households have not applied for a formal loan in the past five years, because the bank branch is too far from their homes or the application procedure involves too much paperwork and waiting time, we categorize them as transaction cost rationed. If instead households do not apply for loans because they do not want to offer their land, house, or other assets as collateral that might be taken by the bank, we consider them risk rationed. Some households that are able to borrow do not apply because they do not need credit; they are also credit unconstrained. Finally, households that would have applied for loan, had they known the bank would lend to them, are another group of supply-side rationed households. We sum together the risk- and transaction cost rationed households into a group of demand constrained 
households; then we sum the demand constrained households and supply constrained households into a larger group of credit constrained households.

\subsection{Data and identification strategy}

\subsubsection{Study setting}

This study took place in the central Rift Valley zone of the Oromia regional state in southeastern Ethiopia. The area is characterized by plain plateaus and low-land agro-ecology. The pattern and intensity of rainfall exhibits considerable spatial and temporal variation, with a bimodal distribution. Rainfall seasons are from May to August and during October and November. However, moisture stress and drought frequently cause devastating crop failure, rampant livestock mortality, and herd collapse (Dercon et al. 2005, Takahashi et al. 2016). Major droughts in the area include the 2015-2016 drought, which has followed historical drought trends during 1973-74, 1983-84, 1991-92, 1999-2000, 2005-06, and 2011-12 (Dercon 2004; Takahashi et al. 2016). Households are smallholders and subsistence farmers who often face drought-induced income shocks that translate into erratic consumption patterns (Biazin \& Sterk 2013; Dercon \& Christiaensen 2011). Formal risk management mechanisms are inaccessible, so informal methods are often used. Ex post shock coping mechanisms being practiced in the area include reducing the frequency of meals, distress livestock sales, halting the purchase of production inputs, forcing pupils to withdraw from school for casual labour, renting land and family labour to local landlords, and seeking wage-based employment on floriculture farms held by foreign investors. These mechanisms, however, are costly, and limited in scope (Dercon 2004).

\subsubsection{IBI in the study area}

In 2013, Japan International Cooperation Agency (JICA) and Oromia Insurance Company (OIC) jointly implemented IBI for crops in the Rift Valley zone of Ethiopia to improve the resilience of households in the face of climate change. The product was designed by CelsiusPro Ag and Swiss Re using satellite rainfall data with $10 \mathrm{~km} \times 10 \mathrm{~km}$ grids for the period 1983-2012. Additional information about the insurance product studied in this chapter is provided in appendix 2. The project was implemented in five districts: Boset, Bora, Ilfata, Adamitullu-Jido-Kombolcha (AJK), and Arsi Negele. The selection of kebeles to be covered in each district was based on several criteria including shortage of rainfall, relevance of the area for crop production and food security, and motivation of kebele leaders and members to adopt IBI. The specific selection process was worked as follows. First, before the initial 
intervention in 2013, the Ethiopian Ministry of Agriculture, OIC and JICA discussed and identified districts in which drought shocks are frequent in Ethiopia. Most of these districts are located in the Rift Valley zone. Second, the three organizations organized focus group discussion with selective representative farmers from each kebele within each of the selected districts. Based on these discussions, kebeles with severe drought experience in the past were identified. Third, as the financial support that JICA allotted for the 2013 weather index insurance intervention was not adequate to cover all the identified drought-prone kebeles at the same time, kebeles for a first intervention in 2013 and kebeles for later interventions were selected by means of discussions between the three organizations. ${ }^{22}$

The product is marketed and sold twice per year during April and during September, months preceding the two rainy seasons, to provide coverage against losses during the seedling and flowering stages of crop growth. Major targeted stable food crops include maize, wheat, barley, and teff. During each sales period, a household that decides to buy IBI pays a premium of $\mathrm{ETB}^{23} 100$ per policy. The payout is determined according to the level of rainfall measured at the meteorological station nearest to the focal kebele for a specified period. If the level of rainfall is below a threshold but above the exit level, ${ }^{24}$ a partial payout of ETB 250 is made. If the decrease in rainfall falls below the exit level, a full payout of ETB 500 is made to policyholders. The exact trigger and exit level differ for each kebele in each season. A markup at $20 \%$ on the actuary fair premium is also included in the contract to cover selling and administration costs (the loading factor). The selling costs refer to costs made by selling agents (e.g., cooperative unions). The actuarially fair premium of the insurance product is ETB 100 per policy for a payout of ETB 500 to cover full losses. The mark-up of $20 \%$ is paid by JICA, so that farmers only pay the actuarially fair premium of ETB 100 per policy. According to OIC sales records, before September 2014 (start of the data collection period),

\footnotetext{
${ }^{22}$ We tried to obtain details about how kebeles that are served first were selected. The official answer of OIC was that this has been done randomly. However, as OIC was not able to inform us about how exactly the randomization was conducted, we doubt whether this is actually the case. It seems more likely that the final selection was very much influenced by the group discussions and based on informal criteria.

${ }^{23}$ ETB (Ethiopian Birr), 1 USD $=20$ ETB as of 2013.

${ }^{24}$ The exit level is the minimum level of rainfall that triggers a payout. A shortage of rainfall, below this level, indicates a catastrophe (higher level of risk) which market-based hedging instruments cannot manage, requiring external assistance such as food aid.
} 
approximately 5000 households in more than 30 kebeles of the five districts had purchased IBI, and plans were in place to expand into many other adjacent kebeles in each district. The intervention plan in subsequent periods involved intensifying coverage of the kebeles in the five districts, then expanding into other districts. Our identification strategy, as explained subsequently, is based on these expansion plans.

\subsubsection{Sampling}

We used a multi-stage random sampling technique with probability proportional to size. More concretely, we first selected three districts (Bora, AJK and Arsi Negele) of the five districts covered by the IBI project. Next, we identified a random sample of kebeles covered by IBI in these three districts. From the AJK district, we selected two kebeles (Qamo Garbi and Desta Abijata) where IBI was not yet available. ${ }^{25}$ Based on our available budget, we decided to survey in total around 1200 households, and to select households, proportional to the sample size of the study, from all kebeles identified. We decided to over-sample households from the treatment region in order to ensure that we will have enough actual "adopters" in our sample. More concretely, we decided to use a sampling strategy that would lead to a sample in which around $70 \%$ of the farmers are in the treatment region and around $30 \%$ in the control (expansion) region. We used random sampling within the treatment and control regions. That is for each kebele we used a roaster of farmers, from which we randomly selected our sample. Note that JICA and OIC organized product promotion campaigns in both the treatment and expansion region to enhance uptake of the index insurance. In both regions, an estimated amount of $70 \%$ of the farmers attended the insurance product promotion meeting. The final sample consists of 1143 households, of which 812 are in a treatment area, and 331 in the control region. Of these 812 households, 459 were adopters, and 353 were non-adopters of IBIs over the period 2013-14.

Table 4.1 presents the mean of adopters and non-adopters both regions. The average number of family members is seven, four of whom have reached productive age, with three who are either pre-school children or age dependents. On average, these households travel for approximately two hours to access public services, including market centres and financial institutions.

\footnotetext{
${ }^{25}$ These two kebeles were identified for future interventions, which will likely take place in coming years.
} 
Table 4.1: Sample characteristics

\begin{tabular}{lccccc}
\hline Variables & Age & Gender & Education & $\begin{array}{c}\text { Dependency } \\
\text { ratio }\end{array}$ & $\begin{array}{c}\text { Distance } \\
\text { from market }\end{array}$ \\
\hline Treatment region uptake & 39.4 & 0.8 & 2.5 & 0.5 & 1.7 \\
Treatment region no-uptake & 38.1 & 0.9 & 2.3 & 0.5 & 1.7 \\
Control region & 37.8 & 0.8 & 2.3 & 0.5 & 1.5 \\
Observations & 1,143 & 1,143 & 1,143 & 1,143 & 1,143 \\
\hline
\end{tabular}

Note: The statistics in Table 4.1 refer to the mean of adopters in Treatment region; the mean of nonadopters in treatment region; and the mean for households in the control (expansion) region.

There are some differences compared to households that did not buy insurance in the treatment area and for households in the control region, but these differences are small. Additional descriptive statistics is provided in Table A2 under Appendix 3.

\subsection{Identification strategy}

A potential bias in our study of the impact of IBI on credit rationing relates to selection bias. Smallholders buy IBI on a voluntary basis, which could result in major differences between adopters and non-adopters of IBI. For example, if more entrepreneurial smallholders decide to adopt IBI, unmeasured variables, such as entrepreneurial spirit, likely affect both the decision to adopt and the probability to obtain credit. A straightforward method to address selection biases would be to conduct intention to treat (ITT) analyses. However, an ITT approach only provides unbiased estimates if the treatment and control regions are similar, and hence that there is no program placement bias. However as we have explained above, the selection of treatment regions and control regions was not entirely random. Thus, ITT estimates may suffer from program placement bias. Nevertheless, it is worthwhile to explore whether ITT analyses could be useful by comparing characteristics of the treatment and control regions. 
Table 4.2: Comparing treatment and expansion regions

\begin{tabular}{lccccc}
\hline Variables & $\begin{array}{c}\text { Full } \\
\text { sample }\end{array}$ & $\begin{array}{c}\text { Treatment } \\
\text { region }\end{array}$ & $\begin{array}{c}\text { Expansion } \\
\text { region }\end{array}$ & $\begin{array}{c}\text { Mean } \\
\text { difference }\end{array}$ & T-stat \\
\hline Population density & 1.227 & 1.266 & 1.133 & -0.133 & -1.837 \\
& $(1.105)$ & $(0.042)$ & $(0.890)$ & $(0.072)$ & \\
Housing quality & 0.642 & 0.606 & 0.731 & 0.125 & $4.030^{* * *}$ \\
Salvage value of assets (net) & $(0.480)$ & $(0.489)$ & $(0.444)$ & $(0.031)$ & \\
& 7360.05 & 7075.87 & 8057.18 & 981.31 & 1.487 \\
Saving (ETB) & $(10121.90)$ & $(7908.05)$ & $(14148.23)$ & $(659.72)$ & \\
PSNP dependent & 1256.99 & 1414.40 & 870.85 & -543.55 & -1.736 \\
Livestock owned in TLU & $(4805.23)$ & $(5217.10)$ & $(3578.41)$ & $(313.09)$ & \\
Wealth index & 0.117 & 0.087 & 0.190 & 0.103 & $4.953^{* * *}$ \\
& $(0.322)$ & $(0.283)$ & $(0.393)$ & $(0.021)$ & \\
Dependency ratio & 9.634 & 9.759 & 9.329 & -0.423 & -0.816 \\
& $(8.069)$ & $(8.097)$ & $(8.004)$ & $(0.526)$ & \\
Access to non-farm employment & 0.546 & 0.478 & 0.713 & 0.235 & $7.408^{* * *}$ \\
& $(0.498)$ & $(0.018)$ & $(0.025)$ & $(0.032)$ & \\
& 0.499 & 0.491 & 0.519 & 0.028 & $2.149 * *$ \\
& $(0.487)$ & $(0.490)$ & $(0.480)$ & $(0.032)$ & \\
\hline
\end{tabular}

$* * * \mathrm{p}<0.01, * * \mathrm{p}<0.05, * \mathrm{p}<0.1 \quad$ Standard deviations are in brackets. Cluster-robust standards errors (clustered at Iddir level).

Table 4.2 compares averages of the treatment and control regions in terms of (1) population density $^{26}$ and (2) different poverty indicators (i.e., housing conditions ${ }^{27}$; being a beneficiary of a productive safety net program (PSNP); a wealth index ${ }^{28}$; the size of the livestock ${ }^{29}$ and an

${ }^{26}$ We measured population density as the ratio of the households' family size to land size. Households living in areas that have dispersed population density may have large land size which may offer them better economic opportunities. We calculate the net size of land possessed or owned by the household from the details of land owned by the household adjusted for land rented-in, rentedout, land share cropped-in and share cropped-out. Comparing the two regions on the basis of the ratio of family size to land provides a good proxy for the "economic implications" of population density. ${ }^{27}$ We proxied housing quality of the households by an indicator variable, indicating whether the household owns an iron corrugated house. In Ethiopia, households construct iron corrugated houses as an improvement from thatch houses.

${ }^{28}$ The wealth index is constructed by using information from the various plant asset compositions owned by households, and the salvage values of each asset in our dataset. 
indicator for 'access to non-farm employment'. Table 4.2 suggests that the treatment and expansion regions differ in terms of a variety of poverty indicators, including housing quality, being PSNP dependent, a wealth index and the dependency ratio. Thus, an ITT approach seems to be problematic, and probably not appropriate. ${ }^{30}$ Therefore, we rely on another methodology.

To reduce selection bias as much as possible, we use an approach in line with a doubledifference methodology. Our approach can be summarized as follows. First, we consider households (adopters and non-adopters of IBI) in areas where IBI are currently available (treatment area). Second, we consider households in areas where IBI is not yet available, but these households represent potential future clients, according to the insurance company (this is our control area; the expansion area for the insurance company). Third, using the sample of households in the treatment area (where IBI is available), we estimate the propensity to adopt and conduct an out-of-sample forecast of 'expected' adopters and expected non-adopters in the control area (the expansion region where IBI is not yet available).

Note that in order to be able to conduct the out-of-sample forecasts, all variables taken into account in the equation to estimate propensities to adopt in the treatment area also need to be (and actually are) available for the expansion (control) region. Fourth, we estimate the impact of IBI on credit rationing by a double-difference methodology in a cross-sectional framework, which includes three steps: (a) comparing predicted adopters and predicted non-adopters in the treatment area. ${ }^{31}$ We use predicted adopters instead of actual adopters as unobserved factors may both increase uptake and affect credit rationing; (b) comparing predicted adopters and predicted non-adopters in the control area, and (c) comparing the differences of a with $b$.

\footnotetext{
${ }^{29}$ The size of livestock owned by the household was measured in a standard unit (TLU).

${ }^{30}$ Nevertheless it may be relevant to present ITT estimates. We therefore present ITT estimates in Table A3 under appendix 3. The ITT estimates suggest that having access to index insurance does not affect overall credit rationing. A further analysis suggests that having access to index insurance enhances supply-side credit rationing and does not significantly affect demand side credit rationing.

${ }^{31}$ In an earlier version of the chapter, we compared in step (a) actual adopters and actual nonadopters in the treatment area, and in step (b) predicted "expected" adopters and predicted nonadopters in the control area, and in step (c) we compared the differences of a with b. We thank an anonymous referee for his/her suggestion to also use the same parameter estimates (the logit uptake model) to predict uptake in the treatment region. The earlier results, using actual adopters in the treated region can be obtained on request.
} 
Basically our approach combines propensity scores with DID. Our approach is innovative as it combines propensity scores with DID to improve identification in case baseline data are not available. To the best of our knowledge, there are no other empirical studies available that have used the same approach to estimate the impact of insurance. ${ }^{32}$ This methodology thus reveals the extent to which adopters in the treatment area are doing better than non-adopters in the treatment area, relative to the difference between predicted adopters and non-adopters in the control area. Our DID approach is similar to the one used in standard DID. While in standard DID a comparison between years and participants and non-participants is made, DID in a cross-sectional setting compares program and non-program villages, and target and nontarget groups (See e.g., Coleman 1999; Armendariz and Morduch 2010 and Khandkar et al. 2010). ${ }^{33}$

In our case, the DID approach involves comparing the difference between outcomes of farmers who take up insurance and farmers who do not take up insurance in areas where insurance is available, with outcomes of farmers who (would like to) take up insurance and farmers who do not (want to) take up insurance in areas where insurance is not yet available. Table 4.3 explains the approach. We consider areas (in our case kebeles) where insurance is available (1) and kebeles without the availability of insurance (2).

In the kebeles with insurance, we know who participates (takes-up insurance: group A) and those who do not participate (group C); for the kebeles without insurance we determine a group that would likely have participated in case the insurance would be available (group D) and the group that would probably not want to participate in case the insurance would be available (group B). By comparing $\mathrm{C}$ with $\mathrm{D}$, selection bias at the household level may be addressed.

\footnotetext{
${ }^{32}$ Cecchi et al. (2016) use a methodology somehow similar to ours. While they do not explore the double-difference methodology, they assess the impact of health insurance on public good contributions by comparing (predicted) adopters and non-adopters across villages with and without access to health insurance.

${ }^{33}$ The methodology we use comes closest to the methodology used by Coleman (1999). In line with his approach, we employ the expansion strategy of the organization, and estimate a double-difference model in space. However, while Coleman had ex-ante information about future adopters in the control region, we lack this information and thus had to estimate future adopters by using an out of sample forecast.
} 
The assumption is that characteristics of participants and (expected) participants are similar, so that the difference between the two reflects the impact of the insurance. Yet, a simple comparison of the means $\mathrm{C}$ and $\mathrm{D}$ does not address potential biases due to non-random program placement, which may be a serious issue if the order in which the insurance company serves the different areas is not random.

Table 4.3: Cross sectional double difference method

\begin{tabular}{llc}
\hline Sample households & $\begin{array}{l}\text { Kebeles with } \\
\text { insurance }\end{array}$ & $\begin{array}{c}\text { Kebeles without } \\
\text { insurance }\end{array}$
\end{tabular}

Non-participants and (would be) non-participants

C B

Participants and (expected) participants

A

$\mathrm{D}$

Note: Based on eq. (4.1), $\mathrm{A}=\mathrm{c}+\beta ; \mathrm{B}=\mathrm{c} ; \mathrm{C}=\mathrm{c}+\alpha+\beta+\gamma ; \mathrm{D}=\mathrm{c}+\alpha$

In order to address non-random program placement, we measure differences-in-differences. Our approach comes down to first comparing $\mathrm{C}$ with $\mathrm{A}$, and then $\mathrm{D}$ with $\mathrm{B}$ and next taking the difference between the two. Hence, the DID estimate is: $\left(Y_{C}-Y_{A}\right)-\left(Y_{D}-Y_{B}\right)$, where $\mathrm{Y}$ represents the outcome variable (access to credit, for instance) and the subscripts A, B, C, D represent the different groups.

We estimate the DID estimator in a regression framework, which enables us to control for other variables and hence reduce the residual variance and improve precision. In a regression framework, the DID approach is implemented as:

$$
Y_{i j}=\mathrm{c}+\alpha W_{i j}+\beta R_{j}+\gamma W_{i j} R_{j}+\delta X_{i j}+\mu_{i j}
$$

where $\mathrm{Y}$ is the outcome variable of interest (in our case, indicators for credit rationing); $\mathrm{W}$ is a binary dummy equal to 1 if it is predicted that the household has adopted (in treatment area) IBI or would adopt IBI (in control area), and 0 if not; $\mathrm{R}$ is a binary dummy equal to 1 for the treatment area and 0 for the control area; $X$ is a vector of household controls; and the subscripts $i$ and $j$ refer to household $i$ in region. ${ }^{35}$ The coefficient of interest is $\gamma$; a significant $\gamma$ indicates that predicted adopters in the treatment area are doing better (or worse, depending on the sign) than predicted non-adopters in the treatment area, relative to the difference between predicted expected adopters and predicted non-adopters in the control area,

\footnotetext{
${ }^{34}$ For reasons of convenience, we specify a linear model to explain our methodology. It should be noted, though, that our estimates are based on non-linear (logit) models, as will be explained below.

35 The coefficients in eq. (1) correspond to conditional means. Specifically, $A=c+\beta ; B=c$; $C=c+\alpha+\beta+\gamma ; D=c+\alpha$. DID estimate then equals: $(C-A)-(D-B)=[(c+\alpha+\beta+\gamma)-(c+\beta)]-[(c+\alpha)-c)]=\gamma$.
} 
controlling for household characteristics $\mathrm{X}$. The parameter $\alpha$ controls for the selection effect, by measuring the extent to which predicted adopters differ from non-adopters in terms of access to credit. With this approach, we assume that the dummy variable $\mathrm{W}$ proxies for the unobservable characteristics that may cause smallholders to adopt IBI (i.e., cause smallholders to self-select into IBI). In line with standard double-difference models, $\alpha$ is a fixed effect for smallholders with the characteristics of adopters. Therefore, $\gamma$ measures the impact of IBI on credit rationing, controlling for the selection effect $\alpha$ and the non-random program placement effect $\beta$. The variable $R$ indicates, for smallholders who have self-selected into IBI, whether IBI is available. A simple regression of credit rationing (Y) on an uptake dummy $(W * R)$ and a vector of controls $X$, ignoring the term $\alpha W_{i j}$, instead would lead to biased coefficients, because the resulting analyses could not control for characteristics that correlate with both uptake and $Y$.

Furthermore, $W$ in eq. (4.1) is a generated regressor, which may lead to biased standard errors if we estimate eq. (4.1) using ordinary least squares (OLS). Therefore, we use a bootstrapping procedure to determine standard errors (Cameron et al. 2008). Specifically, we wrote a small stata.ado program that enables us to estimate eq. (4.1) simultaneously with the procedures to estimate expected adopters in the control region (steps 1-3 in the next section), as well as bootstrap the entire process. More details (and the stata.ado file) are available on request.

\subsection{Credit access in the sample}

Using the direct elicitation method as explained above, we also identify the credit rationing status of each household in our sample. Table 4.4 summarizes the results. It thus appears that about $38 \%$ of the households in our sample are credit constrained, of which $20.5 \%$ are quantity constrained and $18 \%$ are demand constrained. Table 4.4 also differentiates between the regions with and without access to index insurance. It appears that the percentage of households that are credit constrained is higher in the region with access to index insurance, than in the region without access to index insurance. The same holds for the percentage of households who are quantity constrained. However, we hasten to add that this does not necessarily imply that having access to index insurance like an intention to treat (ITT) leads to more credit rationing. The statistics in column 1 in Table 4.4 refer to the entire sample; the second column refers to the treatment region (with index insurance available); the third column refers to the control region (no index insurance available); the last column gives pvalues for a T-test (comparing means of treatment and control Region). 
Table 4.4: Credit rationing in the sample (percentages)

\begin{tabular}{lcccc}
\hline Variables & Entire Sample & $\begin{array}{c}\text { Treatment } \\
\text { Region }\end{array}$ & $\begin{array}{c}\text { Control } \\
\text { Region }\end{array}$ & $\begin{array}{c}\text { T-test (comparing treatment- } \\
\text { control)(p-values) }\end{array}$ \\
\cline { 2 - 5 } & $\mathbf{( 1 )}$ & $\mathbf{( 2 )}$ & $\mathbf{( 3 )}$ & $\mathbf{( 4 )}$ \\
\hline Credit rationed & 0.382 & 0.400 & 0.338 & $0.05(0.26)$ \\
Quantity rationed & 0.205 & 0.228 & 0.148 & $0.002(0.05)$ \\
Demand rationed & 0.178 & 0.172 & 0.190 & $0.47(0.66)$ \\
Number of observation & 1143 & 812 & 331 & \\
\hline
\end{tabular}

The first value refers to a p-value using normal standard errors; the second $p$-value (between brackets) refers to a p-value based on cluster robust standard errors (clustered at kebele level).

\subsection{Identification of expected adopters}

A major challenge of our methodology is identifying both expected future adopters and expected future non-adopters in the control areas. We asked all households in the control areas whether they would like to buy IBI, if it were available, and initially we planned to use their answers to distinguish between expected future adopters and expected future nonadopters. However, as we show in Table A4 in the Appendix 3, comparing the percentage of adopters (treatment area) and expected future adopters (control area) using direct answers from smallholders in the control area seems to lead to a considerable overestimate of expected future adopters. Therefore, we decided to identify adopters in the control area according to estimates of the propensity to adopt in the treatment area, conducted in a three-step procedure.

Step 1: Estimate the propensity to adopt in the treatment area. We consider the decision to buy IBI or not. The utility difference of having an IBI or not depends on the vector of characteristics $Z$. The utility difference for having an IBI or not can thus be written for each household as a function of observed characteristics $Z$ and unobserved characteristics $\varepsilon_{i j}$. Assuming a linear additive relationship, we obtain for the utility difference

$$
W_{i j}^{*}=\beta Z_{i j}+\varepsilon_{i j}
$$

where $W_{i j}^{*}$ is an unobserved latent variable. We assume that the individual buys an IBI if the utility difference exceeds 0 . Consequently: $W_{i j}=1$ (adoption of IBI) if $W_{i j}^{*}>0$, and $W_{i j}=0$ (no adoption of IBI) if $W_{i j}^{*} \leq 0$. We also assume a logistic distribution of the $\varepsilon_{i j}$ values and estimate the equation with a binary logit model (see Table 4.5).

To identify the determinants of uptake, we include a series of variables that other studies have identified as potentially important. Table A5 under Appendix 4 provides more details about these variables. Risk aversion may affect uptake, so we add this variable, derived from an 
incentivized lab-in-the-field experiment that was conducted to mimic the risk attitudes of households. Our risk experiment includes a multiple price list protocol that involves choosing from a safe option and a risky option (50/50 probability) (Binswanger 1980). More details are available on request. Table 4.5 suggests that risk aversion negatively affects uptake, which sounds counterintuitive (Barham et al. 2014; Belissa et al. 2018a). This can be attributed to the fact that the experiment used to measure risk aversion in this case does not differentiate between risk and ambiguity.

We also add an indicator for time preference, derived from a time preference game we played with all households in the sample. The estimate shows that the relationship between the time preference variable and uptake is positive. However, this variable appears to be insignificant. As expected, the more basis risk is expected, the lower the probability to adopt insurance. However, this effect is also insignificant, probably because of its high correlation with the variable intertemporal adverse selection, an indicator that measures whether households are less willing to buy if their uncertainty about insurance payments is larger. ${ }^{36}$ Attending insurance promotion meetings positively affects uptake, as signalled by the positive significant coefficient for insurance product promotion. In addition, households seem more willing to adopt insurance if some of their peers have bought insurance before (peer influence). Older and female household heads exhibit a higher probability of adopting than younger and male-headed households. Table 4.5 presents the results on determinants of uptake including all these variables. The uptake regression appears to be quite good, as reflected, for instance, by the "area under the ROC curve" which equals 0.85 . This implies that our uptake equation is "good" in separating adopters from non-adopters.

Step 2: Using eq. (4.2), as presented in Table 4.5, we conduct an out-of-sample forecast to predict the propensity to adopt in the control area. It should be noted that all variables taken into account in Step 1 are available both for the treatment and the control regions, so that out of sample forecasts are possible. This also holds for the binary variable insurance product promotion as during our survey the insurance company had already organized promotion meetings in the expansion (control) regions.

\footnotetext{
${ }^{36}$ Basis risk becomes significant if the intertemporal adverse selection variable is dropped from the model. The main results hold for three alternative models: (1) both variables included, (2) only basis risk included, or (3) only intertemporal adverse selection included.
} 
Table 4.5: Determinants of uptake in treatment region

Variables

Age

Uptake of IBI

$0.019^{* *}$

(0.009)

District 1

$-0.347$

(0.994)

District 2

$-0.131$

(0.918)

Peer influence

$1.293 * * *$

$(0.374)$

Risk aversion

$-0.542 *$

$(0.279)$

Time preference

0.581

$(0.432)$

Basis risk

$-0.033$

$(0.064)$

IBI product promotion

$2.886^{* * *}$

Gender

$-0.545$

$(0.348)$

Intertemporal adverse selection

$0.602 * *$

(0.276)

Constant

$-2.916^{* * *}$

(0.958)

Area under ROC

0.85

Correctly classified

$79.7 \%$

Sensitivity $(\operatorname{Pr}(+\mid D))$

$87.8 \%$

Specificity $(\operatorname{Pr}(-\mid \sim D))$

$69.1 \%$

Pseudo R ${ }^{2}$

Observations

812

Standard errors, in parentheses, were obtained by conducting a cluster bootstrapping method (with 'kebele' as the cluster variable) on a system of equations, including the uptake equation and the creditrationing eq. (1). ${ }^{* * *} \mathrm{p}<0.01{ }^{* *} \mathrm{p}<0.05{ }^{*} \mathrm{p}<0.1$. Sensitivity refers to the percentage of actual adopters that have a propensity of adoption above 0.5; Specificity refers to the percentage of actual non-adopters that have a propensity of adoption below 0.5 .

Step 3: For the identification of expected future adopters and expected future non-adopters in the control area, we classify households in the control area with a probability to adopt above 
or equal to 0.5 as expected adopters; expected non-adopters have a probability to adopt that is less than 0.5 .

Step 4: For reasons outlined above, we also reclassify adopters in the treatment area using this methodology. Table 4.6 compares the percentage of predicted and actual adopters in the treatment area with the predicted expected adopters in the control area. The percentages appear similar, which suggests that our logit estimates provide reasonably accurate estimates of expected adopters. Our assumption a priori was that the percentages of adopters would not differ enormously between the treatment and control regions.

Table 4.6: Adopters and non-adopters by area

\begin{tabular}{lcccc}
\hline & Treatment area & Percentage & Control area & Percentage \\
\hline Actual Adopters & 459 & 56.5 & - & \\
Predicted Adopters & 512 & 63 & 195 & 59 \\
Actual Non-adopters & 353 & 43.5 & - & - \\
Predicted Non-adopters & 300 & 37 & 136 & 41 \\
Total & 812 & 100 & 331 & 100 \\
\hline
\end{tabular}

Note: proportion of adopters and non-adopters, actual and predicted, in treatment and control areas.

\subsection{Selection (balance) tests}

To test the reliability of our methodology, we conduct some selection tests and investigate whether the selection process is similar between the treatment and control regions. The selection tests need to be done on variables that have not been affected by the actual and predicted uptake of IBI (i.e., exogenous variables), and hence should also not include variables that are included in the uptake equation. The selection tests need to consider characteristics of households that bought and did not buy IBI. We therefore need to consider $\left(X_{C}-X_{A}\right)-\left(X_{D}-X_{B}\right)$, where $X$ represents exogenous variables and the subscripts $A, B, C$, $D$ refer to the different groups, as specified above. In a regression framework this comes down to estimating

$$
X_{i j}=\mathrm{c}+\alpha W_{i j}+\beta R_{j}+\gamma W_{i j} R_{j}+\mu_{i j}
$$

where $\alpha$ captures differences between predicted adopters and predicted non-adopters, and $\beta$ captures differences between the regions with and without access to IBI. $W$ is a dummy variable with a 1 if the farmer is predicted to be an adopter of IBI. $R$ is a region dummy with a 1 if IBI is already available in the region. The parameter of interest is $\gamma$. It captures differences 
between adopters and non-adopters beyond differences that are already taken into account by $\alpha$ and $\beta$. An insignificant $\gamma$ implies that the selection process in the two areas is similar in terms of observables. Balancing tests refer to results obtained using eq. (4.3) and these are presented in Table 4.7.

Table 4.7: Selection (balance) tests

\begin{tabular}{llllllc}
\hline Variables & $\begin{array}{c}\text { Education } \\
\text { (years) }\end{array}$ & $\begin{array}{c}\text { Family } \\
\text { size }\end{array}$ & $\begin{array}{c}\text { Extension } \\
\text { Contract }\end{array}$ & $\begin{array}{c}\text { Coop } \\
\text { member }\end{array}$ & Dependents & $\begin{array}{c}\text { Draft } \\
\text { oxen }\end{array}$ \\
\hline Region.( R) & -0.65 & $-0.63^{* *}$ & 0.05 & $0.04^{* *}$ & -0.30 & -0.31 \\
Uptake (W) & $-0.84^{* *}$ & $0.84^{* *}$ & 0.03 & $0.09^{* * *}$ & $0.62^{* *}$ & 0.69 \\
Region*Uptake & $1.89^{* * *}$ & -0.01 & -0.02 & -0.01 & -0.26 & -0.14 \\
$(\mathrm{R} * \mathrm{~W})$ & & & & & & \\
Constant & $4.25^{* * *}$ & $6.94^{* * *}$ & $0.92^{* * *}$ & $0.00^{* * *}$ & $3.61^{* * *}$ & $1.85^{* * *}$ \\
Observations & 1143 & 1143 & 1143 & 1143 & 1143 & 1143 \\
\hline $\mathrm{R}^{2}$ adjusted & 0.02 & 0.03 & 0.01 & 0.02 & 0.02 & 0.02
\end{tabular}

Note: $\mathrm{p}$-values based on cluster robust standard errors with kebeles as cluster; ${ }^{*} \mathrm{p}<0.10 ;{ }^{*} \mathrm{p}<0.05$; $* * \mathrm{p}<0.01$

As Table 4.7 shows, with the exception of the years of education, all variables are balanced, which provides additional confidence in our approach, and thus for our identification strategy. The adopters in the treatment region apparently enjoy a bit more education than expected adopters in the control region. ${ }^{37}$ To control for possible biases due to this difference, we add education (years) as another control variable in the credit rationing regressions.

\subsection{Main results}

\subsubsection{Impact of IBI on credit rationing}

Table 4.8 presents descriptive statistics regarding credit rationing for the four groups: predicted adopters and non-adopters in the treatment and control region. Using the double difference approach as set out in Table 4.3, the results presented in Table 4.8 suggests that after controlling for selection effects and program placement effects, the adoption of IBI

\footnotetext{
${ }^{37}$ It should be noted that for large samples a t-test - and hence also our approach - may reveal imbalances, even if the differences are small. Alternatively, when the amount of observations is very small, the null hypothesis of equal means may not be rejected even if differences are relatively big. Therefore, testing for balance requires to consider the magnitude of the coefficient. In our case, the years of education is about 1-2 years longer for adopters in the treatment area, which seems relatively small. Imbens (2015) suggests using normalized differences as an alternative test for balance.
} 
would reduce overall credit rationing by $18 \%$ (i.e., $[0.39-0.42]-[0.40-0.25]=-0.18$ ). Table 4.8 also clearly shows that determining the impact of IBI by comparing credit rationing status of predicted adopters in the treatment and control region provides a biased estimate: a simple comparison of predicted adopters suggests that IBI reduces credit rationing by only $1 \%(0.39$ 0.40). However, by comparing credit rationing of predicted non-adopters in both regions it become clear that there is a regional effect (a program placement bias) equal to $17 \%(0.42$ 0.25 ), so that the impact of IBI, controlling for selection bias and the region effect equals $18 \%$.

Table 4.8: Credit rationing for predicted adopters in treatment and control region

\begin{tabular}{lcccccc}
\hline Variables & \multicolumn{3}{c}{ Treatment Region } & \multicolumn{3}{c}{ Control Region } \\
& $\begin{array}{c}\text { Predicted } \\
\text { adopters }\end{array}$ & $\begin{array}{c}\text { Predicted non- } \\
\text { adopters }\end{array}$ & Total & $\begin{array}{c}\text { Predicted } \\
\text { adopters }\end{array}$ & $\begin{array}{c}\text { Predicted non- } \\
\text { adopters }\end{array}$ & Total \\
\hline Credit rationed & 199 & 126 & 325 & 78 & 34 & 112 \\
Quantity rationed & $(0.39)$ & $(0.42)$ & $(0.40)$ & $(0.40)$ & $(0.25)$ & $(0.34)$ \\
& 112 & 73 & 158 & 35 & 14 & 49 \\
Demand rationed & $(0.22)$ & $(0.24)$ & $(0.23)$ & $(0.18)$ & $(0.10)$ & $(0.15)$ \\
& 87 & 53 & 140 & 43 & 20 & 63 \\
\hline Observations & $(0.17)$ & $(0.18)$ & $(0.17)$ & $(0.22)$ & $(0.15)$ & $(0.19)$ \\
\hline
\end{tabular}

Note: The statistics in Table 4.8 shows total number of households and the percentage (in parenthesis) for each category.

We further assess the impact of IBI on credit rationing in general by using eq. (4.1), as specified in Table 4.9, according to a binary logit estimate. ${ }^{38}$ Table 4.9, first column (Overall Credit rationing) presents the results. The coefficient of interest refers to $\mathrm{W} \times \mathrm{R}$. This equation is estimated simultaneously with eq. (4.1).

\footnotetext{
${ }^{38}$ According to theory, IBI could also induce lenders to reduce interest rates. However, for our survey area we did not find any evidence supporting this claim. Hence, it seems as if lenders do not properly price risk.
} 
Table 4.9: Impacts of IBI uptake on credit rationing

\begin{tabular}{|c|c|c|c|}
\hline Variables & $\begin{array}{l}\text { Overall credit } \\
\text { rationing }\end{array}$ & $\begin{array}{c}\text { Supply-side } \\
\text { credit } \\
\text { rationing }\end{array}$ & $\begin{array}{l}\text { Demand- } \\
\text { side credit } \\
\text { rationing }\end{array}$ \\
\hline \multirow[t]{2}{*}{ Region (R) } & $1.109 * * *$ & $1.615^{* * *}$ & 0.539 \\
\hline & $(0.303)$ & $(0.358)$ & $(0.476)$ \\
\hline \multirow[t]{2}{*}{ Uptake (W) } & $0.774 *$ & $0.852 * * *$ & 0.722 \\
\hline & $(0.347)$ & $(0.391)$ & $(0.597)$ \\
\hline \multirow[t]{2}{*}{ Region*Uptake (W*R) } & $-0.867 * *$ & $-1.109 * *$ & -0.617 \\
\hline & $(0.363)$ & $(0.440)$ & $(0.611)$ \\
\hline \multirow[t]{2}{*}{ Age } & -0.002 & 0.002 & -0.005 \\
\hline & $(0.006)$ & $(0.008)$ & $(0.008)$ \\
\hline \multirow[t]{2}{*}{ Education (years) } & 0.007 & 0.025 & -0.014 \\
\hline & $(0.019)$ & $(0.024)$ & $(0.025)$ \\
\hline \multirow[t]{2}{*}{ Family size } & 0.012 & 0.014 & 0.013 \\
\hline & $(0.062)$ & $(0.078)$ & $(0.084)$ \\
\hline \multirow[t]{2}{*}{ Extension contact } & 0.534 & 0.837 & 0.318 \\
\hline & $(0.379)$ & $(2.184)$ & $(0.752)$ \\
\hline \multirow[t]{2}{*}{ District 1} & 0.049 & $-1.753 * * *$ & $1.772 * * *$ \\
\hline & $(0.234)$ & $(0.535)$ & $(0.443)$ \\
\hline \multirow[t]{2}{*}{ District 2} & $0.757 * * *$ & $0.481 * *$ & $1.650 * * *$ \\
\hline & $(0.192)$ & $(0.219)$ & $(0.427)$ \\
\hline \multirow[t]{2}{*}{ Dependency ratio } & 0.130 & 0.040 & 0.246 \\
\hline & $(0.791)$ & $(1.023)$ & $(1.043)$ \\
\hline \multirow[t]{2}{*}{ Dependents } & -0.096 & -0.084 & -0.112 \\
\hline & $(0.119)$ & $(0.152)$ & $(0.162)$ \\
\hline \multirow[t]{2}{*}{ Coop member } & $-0.499 * *$ & -0.396 & -0.684 \\
\hline & $(0.258)$ & $(0.313)$ & $(0.421)$ \\
\hline \multirow[t]{2}{*}{ Constant } & $-2.132 * *$ & -3.233 & $-3.148 * * *$ \\
\hline & $(0.634)$ & $(2.259)$ & $(1.094)$ \\
\hline Observations & 1,143 & 1,143 & 1,143 \\
\hline
\end{tabular}

Notes: Standard errors, in parentheses, are based on a bootstrap method, where the bootstrap is performed for a system of equations, containing the uptake eq. (4.2) and either the overall credit rationing equation or the supply and demand-side credit rationing equations. The overall credit rationing equation is estimated with a logit model; the demand-side and supply-side credit rationing equations follow from a multinomial logit regression. The bootstrap relies on 2000 repetitions. ${ }^{* * *} p<$ $0.01 . * * p<0.05 . * p<0.1$.

The results under the first column suggest that farmers who adopt IBI are less credit rationed, as indicated by the significant negative coefficient for the interaction term. The estimated 
parameter value for the interaction term is -0.867 , indicating that for a 1 unit increase in IBIadoption, there is a 0.876 decrease in the log-odds of being credit rationed, holding all the other covariates constant. Alternatively, as IBI adoption increases by 1 unit, the log-odds of being a credit de-rationed household increases by 0.867 .

We also conducted estimations using a linear probability model (LPM). This is undertaken as a simple OLS estimate of eq. (4.1), which is easier to interpret in terms of economic significance. Note that an OLS estimate without any additional controls provides the double difference result of a decrease in credit rationing of $18 \%$. If we add the full set of controls, the OLS estimate suggests that adoption of IBI reduces overall credit rationing even with $19 \%$. Thus, the purchase of index insurance seems to have a sizeable effect on the reduction of overall credit rationing.

\subsubsection{Credit supply and demand-side rationing}

Recall that the impact of IBI on credit rationing may be due to a decrease in demand-side rationing or a decrease in quantity rationing. Therefore, we further investigate the impact of IBI by differentiating between demand- and supply-side constraints. More concretely, to assess the impact of IBI on credit demand- and supply-side rationing, we use a multinomial logit model. Table 4.9 also presents the multinomial logit results, with credit unconstrained households as the base. As this table shows, uptake of IBI significantly reduces supply-side rationing. The estimated parameter value for the interaction term is -1.109 for supply-side credit rationing, and this is significant at a 5 percent level. This indicates that for a 1 unit increase in IBI-adoption, the multinomial log-odds for being credit constrained (relative to credit unconstrained) decreases by 1.109 . A better interpretation of the impact of adopting insurance on being supply-side credit rationed can be obtained by exponentiating the coefficient. Exponentiating 1.109, we obtain a value of 3.03. This implies that the relative

probability (also called the relative odds) of being non-credit rationed rather than being supply-side credit rationed for IBI holders is more than three times the corresponding relative probability for non-IBI holders with the same characteristics. A potential reason for this can be that lending institutions may extend more credit supply for insured households since such households may earn indemnity payments from IBI which can enhance their ability to repay back the loan. That means loan applications of insured households may get better acceptance by lenders. There is also a negative effect of IBI adoption on demand-side credit rationing. 
However, the impact on demand-side credit rationing is not significant at the usual significance levels to deal with its economic relevance.

\subsection{Robustness analyses}

The main results suggest that farmers who adopt IBI are less credit rationed, and that uptake of IBI negatively affects both supply-side and demand-side credit rationing. However, only the impact on supply-side credit rationing seems to be significant. Our methodology may suffer from some limitations, which may affect the main results, though. We undertake various robustness tests by classifying the main potential limitations/biases into six groups as follows: (i) Biases due to wrong impact estimates of insurance on credit rationing (i.e., possible imprecise and biased prediction of adopters in the control region; (ii) Imprecise specification of the credit-rationing equation; (iii) Endogeneity bias and reverse causality; (iv) Biased standard errors of the estimates due to clustering effects; (v) Remaining program placement effects and (vi) Spill-over effects from adopters to non-adopters. By conducting several robustness tests we can assess the importance of most of the above-mentioned potential biases. Below we therefore describe results of a series of robustness tests. Full results of these robustness tests are provided in different tables under appendix 5.

\subsubsection{Biases from imprecise prediction of adopters}

Our approach is based on the assumption that selection effects due to unobservables can be controlled for by adding a dummy variable that indicates which farmers are adopters and would-be adopters. However, if our results turn out to be very sensitive to a misclassification of (would-be) adopters, selection effects may still seriously bias results. In order to get some idea about how serious this limitation might be, we conduct some robustness tests. We start by considering to what extent the results are sensitive to the precise specification of the uptake equation. More specifically, it may be the case that some important determinants of uptake are ignored, leading to omitted variable bias. Moreover, some included determinants of uptake may be affected by insurance, leading to endogeneity bias.

We consider two alternative equations for the uptake equation in order to reduce possible omitted variable bias. First, some additional control variables are included. More specifically, we add four exogenous variables: Education, Family Size, Cooperative membership and Draft Oxen. Next, we ignore 'peer influence' as it may be argued that 'peer influence' is affected by insurance: if somebody buys IBI, there are potentially more peers available that may positively affect other purchasers or non-purchasers. 
Table A6 under Appendix 5 shows that the performance of the alternative uptake equations, in terms of $\mathrm{R}^{2}$ and ROC curve, are similar to the uptake equation presented in Table 4.5. Most importantly, the change in the uptake equations did not qualitatively change the main results in terms of the impact of IBI on credit rationing (see Table A7 under Appendix 5). Also with the alternative specifications of the uptake equation, IBI uptake has a negative impact on both supply-side and demand-side credit rationing. Moreover, it again appears that only the effect on supply-side credit rationing is significant. This impact of insurance on supply-side credit rationing still seems to be large, although somewhat smaller than in the base model.

It may also be the case that prediction of adopters is bad due to very little discriminatory power in the model, possibly even implying that uptake is as good as random. For the base model, we have provided some evidence that discriminatory power of our uptake model is quite good by presenting the (area below the) ROC curve. Yet, for the identification of expected adopters and expected non-adopters in the control area, we classified households in the control area with a probability to adopt above or equal to 0.5 as expected adopters. This is, however, a somewhat arbitrary threshold and it does not guarantee that we will not end up having eventual non-purchasers in our group of likely purchasers. Therefore, we also estimate the model by endogenously setting the threshold value at a level which optimizes the socalled Youden Index (Youden 1950). The Youden Index estimates the probability of an informed decision, contra a random guess, and thus provides a measure for discriminatory power. The index is calculated as: sensitivity + specificity -1 , where sensitivity (or true positive rate) measures the proportion of positives that are correctly classified and specificity (or true negative rate) measures the proportion of negatives that are correctly classified (Imbens 2003). If the model would perfectly predict farmers with and without insurance, sensitivity and specificity would take on the values of $100 \%$ and $100 \%$ respectively. Thus, the Youden Index is defined between -1 and 1. It gives the value of 0 when a test is deemed useless (i.e. when the classification model gives the same proportion of positive results for farmers with and without insurance).

The cut-off point that corresponds with the maximum value of the Youden Index optimizes the performance of the model in terms of the combination of specificity and sensitivity. Our uptake equation provides a maximum value of the Youden index of 0.61 , which corresponds to a cut-off value of 0.666 . As can be seen in Table A8 under Appendix 5, setting the cut-off value at 0.666 does not change the main results qualitatively: still insurance reduces supply- 
side rationing significantly (even more pronounced than in the base model), while it does not significantly affect demand-side credit rationing.

In alternative regressions, we also estimated the model by exogenously declining the cut-off value to values below 0.5 . It turned out that results start to change seriously if the cut-off value drops below 0.4: insurance still reduces credit rationing, but now the effect changes from a significant decline in supply-side credit rationing to a significant decline in demandside credit rationing. While there is no reason to set the cut-off value at a level below 0.5 , as this would deteriorate the performance of the uptake model in terms of prediction errors, the outcome signals that results are quite sensitive to the (predicted) classification in adopters and non-adopters in the control region.

There are several other reasons why prediction of adopters in the control region, based on outof-sample forecasts, may be incorrect. For instance, it may be the case that households in the control region changed their behaviour in expectation of receiving IBI in the near future. While we cannot control for this potential limitation, we have no indication that this happened and hence do not think that our results are biased due to behavioural changes in anticipation of future access to insurance.

\subsubsection{Misspecification bias in credit-rationing}

The results may also be affected by taking into account the wrong set of controls in our outcome equation. We therefore assess whether our results are sensitive to a change in the set of controls added to the credit rationing equations. More specifically, we first estimate an equation without any controls. Moreover, as $W$ is affected by variables taken into account in the uptake equation, we also estimate an equation in which, in addition to the controls specified in the base model, all other variables from the uptake equation are added to the credit rationing equation. Table A9 under Appendix 5, suggests that our main results are hardly affected by changing the set of controls in the credit rationing equation. For both equations, without any controls and with an extended set of controls, the main result still holds: insurance only has a significant effect on supply-side credit rationing. Moreover, the coefficient of interest hardly changes.

\subsubsection{Endogeneity and reverse causality}

A possible limitation of our approach is that the identification strategy may not entirely rule out endogeneity (e.g., due to reverse causality). Several studies suggest that credit constraints 
may reduce insurance demand (e.g., Giné et al. 2008; Cole et al. 2013). This may especially be problematic if (unobserved) characteristics are both related to credit and the variables that affect uptake. For instance, it may be the case that having access to credit increases the likelihood to attend an IBI product promotion meeting, while attending such a meeting increases uptake of insurance. While endogeneity bias may indeed play a role, we do not think that it will nullify our main results. Endogeneity bias (e.g., due to unobserved heterogeneity) is reduced by using "predicted" probabilities of uptake both in the treatment and control regions for classifying (would be) adopters, rather than actual uptake rates. Moreover, as has been shown above, our main results are not affected by ignoring the variable 'peer influence', which may be affected by insurance. Finally, we examined whether being credit rationed affects uptake of IBI, and whether adding a dummy for credit rationing to the uptake equation affects the main results. Table A10 under Appendix 5 shows that our credit rationing dummy is highly insignificant in the uptake equation, and therefore as expected, it does not affect the credit rationing regressions (see Table A11 under Appendix 5). Although the robustness tests we present do not fully control for endogeneity bias, they do give some confidence that reverse causality will not change our main results.

\subsubsection{Bias in standard errors due to clustering effects}

In all models so far, we presented standard errors obtained from a bootstrapping method, without clustering. However, as the introduction of IBI takes place at the kebele level, there may be cluster effects within kebeles, possibly leading to biased standard errors. Therefore, we also estimated all models using the same bootstrapping method, with clustering at the kebele level. As an alternative, we also re-estimated all models using a cluster bootstrapping approach where we cluster at the Iddir level. It turned out that none of the results are significantly affected by using the cluster bootstrapping approach. In Table A12 under Appendix 5, we present results for the impact of insurance on supply-side and demand-side credit rationing using our base specifications for the uptake equation and the credit rationing equation, but with standard errors obtained from the cluster bootstrap approach.

\subsubsection{Unaccounted program placement effects}

The index insurance we consider is introduced at the kebele level. If the order in which kebeles receive IBI is not random, and for instance the most dynamic kebeles first receive treatment, our estimates may be biased. We conduct estimates including kebele fixed effects, 
using a standard bootstrapping approach (see Table A13 under Appendix 5). ${ }^{39}$ It appears that our results are not seriously affected by program placement effects as the main results still hold. The estimation results now even suggest a somewhat more substantial impact of insurance on supply-side credit rationing. Yet, it should be noted that the kebele fixed effects only control for unobservables that affect all households in a kebele identically. If programs target their beneficiaries at qualities specific to target groups within districts or kebeles, fixed effects can actually increase the bias (see Armendariz \& Morduch 2010).

\subsubsection{Spillover effects}

Our methodology assumes that there are no spill overs within IBI areas. If such spill overs exist, they will show up as increases in the overall welfare of the district and/or kebele. These effects will be captured by the district fixed effects (in the main model), or the kebele fixed effects (in the robustness estimates), rather than by program effects. The implication of spill overs from IBI to non-IBI holders in the treatment area is that treatment effects will be biased. Unfortunately, cross-sectional data does not allow us to control for possible biases due to spill overs. Yet, it seems likely that, if spill overs exist, the average impact of index insurance on credit rationing will be underestimated.

\subsection{Conclusion}

Index-based insurance is increasingly recognized as a climate risk management strategy that can benefit the poor. This chapter focuses on an issue that has not received much attention in prior literature, namely, does the adoption of index insurance improve access to financial markets and reduce credit rationing? Our empirical analyses focus on Ethiopia and leverage the situation in which IBI is not yet available in some areas. With an identification strategy, in line with a cross-sectional double-difference method, we try to control for potential selection biases by forecasting potential insurance adopters in the control region. Our identification

\footnotetext{
${ }^{39}$ Note that introducing kebele fixed effects is not possible together with a cluster bootstrapping method where we cluster at the kebele level, because the cluster bootstrap draws clusters with replacement, implying that there will be an insufficient amount of observations. Hence, we can only introduce kebele fixed effects if we use a standard bootstrap method or a cluster bootstrap method at a lower level, such that the amount of clusters exceeds the amount of kebele fixed effects. Yet we have also estimated models with Kebele fixed effects combined with a cluster bootstrapping approach, where we cluster at the Iddir level. The results, which can be obtained on request, are similar.
} 
strategy enables us to draw some conclusions regarding the causal relationship between adopting index-based insurance and credit rationing.

Regarding credit rationing, we differentiate supply-side and demand-side forms. Our impact regressions indicate that IBI reduces both supply-side rationing (quantity rationing) and demand-side credit rationing. However, the latter appears to be statistically insignificant. The study suggests that the impact of IBI uptake on reducing supply-side credit rationing is relatively large. The variety of regressions suggests that the relative probabilities of being non-credit rationed rather than being supply-side credit rationed for IBI holders is more than double the corresponding relative probability for non-IBI holders with the same characteristics. A more straightforward interpretation of the results suggests that index insurance, controlled for selection biases and region effects, reduces total credit rationing by some $18 \%$, while it reduces supply-side credit rationing with some $10 \%$. This shows that adoption of IBI could enhance smallholders' access to credit. It also provides mutual benefits to both farmers (borrowers) and lenders. The benefits to the smallholder are two-fold. First, the alleviation of supply-sided constraints can enable famers to acquire inputs and enhance productivity. Second, as access to credit overcomes liquidity constraints of farmers, this helps them to employ other risk management strategies to hedge against down-side production risk. Lenders also benefit from lending to insured farmers as the default risk of lending to insured borrowers tends to be lower. Hence, the complementary nature of IBI and credit also make borrowers and lenders better off in this context. We accordingly hope that this study encourages new research that seeks to test whether our results also hold in other settings and are robust to other identification strategies, such as the use of randomized controlled trials.

\section{Appendix 2: Additional information about the Index Insurance Product}

So far, two IBI products were implemented in the study area. These are termed as weather index crop insurance (WICI) and vegetation index crop insurance (VICI). WICI was an initial IBI product co-implemented by JICA and OIC in 2013. It was designed by CelsiusPro Ag and Swiss Re, using satellite rainfall data with $10 \mathrm{~km} \times 10 \mathrm{~km}$ grids for the period 1983-2012. OIC used this product during the period 2013-2014 ${ }^{40}$. In WICI, payout is determined according to the level of rainfall measured at the meteorological station nearest to the focal kebele for a specified period. Since 2015, however, OIC further collaborated with the University of Twente in Netherlands and Kifiya Financial Technology in Ethiopia, to design

\footnotetext{
${ }^{40}$ The underwriting procedure includes awareness creation, grid selection, enrolment and sells.
} 
the VICI. VICI is a modified product with better quality compared to the WICI. VICI is designed based on the intensity of vegetation cover or greenery on the earth's surface. Greenery level is measured by a satellite indicator known as normalized difference vegetation index (NDVI) ${ }^{41}$. In VICI design, NDVI is extracted at a geospatial resolution of $1 \mathrm{~km} \times 1 \mathrm{~km}$. The current VICI product used by OIC is based on average NDVI of 16 years. NDVI reflects the already accumulated result of rain on crop growth. It is a primary measurement with no assumptions or calibrations. It is the proven standard index, in use by all early warning units globally. Actual decal NDVI data for a given period is calculated for a set of households grouped in a one crop production system (CPS) zone. The NDVI compiled for grids of $1 \mathrm{~km} \times$ $1 \mathrm{~km}$ is then arranged in percentile ranges from 1 to 20,25 and 50. Based on these percentiles, benchmark values for trigger and exit index points which will be compared to the actual risk level are determined ${ }^{42}$. Quality improvement is reflected in determination of the IBI premium and payout, which is based on some algorithms for both WICI and VICI. Accordingly, VICI is a product with better quality than WICI. To explain this, suppose that for a single policy the household pays a premium amount of $P$ in expectation of a payout $I$ for the total value of sum insured $S$. In the design of WICI, OIC assumed that on average one out of five farmers can incur a loss in a given insurance period. Hence, $P$ is 20 percent of $S$. Based on this, the sum to be insured per policy is given as follows:

$$
S_{\text {wici }}=\frac{P}{0.20}
$$

\footnotetext{
${ }^{41}$ NDVI is measured through images obtained from a geo-satellite weather infrastructure known as GeoNetCast System. The system reads to see if the observed area contains live green vegetation or not. The data from these images are converted into digital numbers (DN-values), i.e. Integers from 0 to 255 creating the NDVI.

${ }^{42}$ In summary, the mechanics of the VICI product design has the following characteristics. The index is based on greenness level. The source of the satellite data is a weather infrastructure known as GeoNetCast. The system converts satellite images into digital numbers ranging from 0 to 255 . It uses NDVI data for the last 16 years and reads deviation from this long-term average. In order to deal with transaction costs, it divides the geographical coverage into CPS zones. NDVI is computed for each zone at grids of $1 \mathrm{~km} \times 1 \mathrm{~km}$. NDVI data are usually arranged in percentiles, and payout is calculated for a decal or every 10 days period. Hence, trigger and exit thresholds are computed for CPS zones and 10-day period specific. The insurance coverage period is crop growth season specific. Payout is not crop-specific, but $1 \mathrm{~km} \times 1 \mathrm{~km}$ grid (location) specific.
} 
However, in the design of VICI, it is assumed that since uptake gradually increases, it is possible to pool more risks across areas with greater geo-spatial variations that can help to reduce transaction costs. Hence, OIC expects nearly about one out of six households who purchased IBI may face losses. Hence, the sum to be insured per policy is given as follows:

$$
S_{\text {vici }}=\frac{P}{0.15}
$$

During each sells window, a household that decides to buy IBI pays a premium of ETB ${ }^{43} 100$ per policy. Payout which is a maximum of sum insured is determined according to the level of the index (i.e., rainfall index for WICI or NDVI index for VICI). Let $T, E$ and $A$ represent trigger, exit and actual parametric values of the indices, rainfall index or NDVI. Then, the amount of payout in each insurance period can be calculated for individual WICI buyer households as follows:

$$
I_{w i c i}=\left(\frac{T-A}{T-E}\right)\left(\frac{P}{0.20}\right)
$$

If the level of rainfall is below a threshold but above the exit ${ }^{44}$ level, a partial payout of ETB 250 will be made. If the decrease in rainfall falls below the exit level, a full payout of ETB 500 is made to policyholders. This means, in WICI only a partial or full payout is made. In the design of VICI, however, payout calculation procedures are based on every 10 days NDVI data generated from the GeoNetCast System. Based on the NDVI data, payout is precisely determined as follows:

$$
I_{v i c i}=\left(\frac{T-A}{T-E}\right)\left(\frac{P}{0.15}\right)
$$

In computing payouts for VICI purchasers, OIC uses a linearly proportional indemnification (LPI) approach rather than the partial or full compensation approach previously used in WICI. For instance, for a single insurance with premium of ETB 100, the payout for a complete loss

\footnotetext{
${ }^{43}$ ETB (Ethiopian Birr), 1 USD $=20$ ETB in 2013/14

${ }^{44}$ The exit level is the minimum level of rainfall that triggers a payout. A shortage of rainfall, below this level, indicates a catastrophe (higher level of risk) which market-based hedging instruments cannot manage, requiring external assistance such as aid. As common parameters of NDVI, trigger point is the level of the index from the weather data at which payout begins while exit point is the index level at which $100 \%$ payout begins.
} 
is 100/0.15 which is about ETB 667. Using the linear proportional partial loss indemnification approach, for instance, in areas where the index indicates a 50\% loss, a partial payout of about ETB 333.5 is paid to the farmers. Hence, with the same premium of Birr 100, the payout for a complete loss is Birr 167 higher for VICI adopters than WICI adopters. In addition to the above technological innovation to improve the quality of IBI, institutional and contractual innovations were attempted to reduce basis risk. Initially, OIC conducted a twin-tracking system to assess the performance of IBI in 2016. Payout per the satellite index was between $12 \%-68 \%$ while payout as per the field assessment is $10 \%-70 \%$, indicating a good correlation between the losses indicated by the index and the actual losses revealed at the ground, and pointing a better quality of the IBI product in the study area.

\section{Appendix 3: Additional estimates}

Table A2: Additional descriptive statistics
(1)
(2)
(3)

(4)

(5)

Variables

Family size Coop member

Peer influence

Basis risk Draft oxen

\begin{tabular}{lccccc}
\hline Treatment region uptake & 7.3 & 0.1 & 0.9 & 2.5 & 2.1 \\
Treatment region no-uptake & 6.2 & 0.1 & 0.6 & 2.5 & 1.6 \\
Control region & 7.4 & 0.1 & 0.5 & 1.3 & 2.3 \\
\hline Observations & 1,143 & 1,143 & 1,143 & 1,143 & 1,143
\end{tabular}

Note: The statistics in Table A2 refer to the mean of adopters in Treatment region; the mean of nonadopters in treatment region; and the mean for households in the control (expansion) region.

Table A3: ITT estimates

\begin{tabular}{lccc}
\hline Variables & $\begin{array}{c}\text { Overall Credit } \\
\text { rationing }\end{array}$ & $\begin{array}{c}\text { Supply-side Credit } \\
\text { Rationing }\end{array}$ & $\begin{array}{c}\text { Demand-side Credit } \\
\text { Rationing }\end{array}$ \\
\hline Region $(\mathrm{R})$ & 0.06 & $0.08^{* *}$ & -0.02 \\
Constant & $0.34^{* * *}$ & $0.15^{* * *}$ & $0.19^{* * *}$ \\
Observations & 1143 & 1143 & 1143 \\
\hline $\mathrm{R}^{2}$ adjusted & 0.00 & 0.01 & 0.00 \\
\hline
\end{tabular}

Note: P-values are based on cluster robust standard errors with kebeles as cluster; ${ }^{*} \mathrm{p}<0.10 ;{ }^{* *} \mathrm{p}<0.05$; $* * * \mathrm{p}<0.01$. The regressions refer to Ordinary Least Squares (OLS) regressions. We have also estimated an ITT model with a nominal logit model (combining the different categories, no constraints, supply-side constraints and demand-side constraints), which suggests, qualitatively, the same results (more supply-side constraints in treatment region) 
Table A4: Adopters and non-adopters by area (direct answers)

\begin{tabular}{lcccc}
\hline & Treatment area & Percentage & Control area & Percentage \\
\hline Adopters & 461 & 56.78 & 287 & 87.00 \\
Non-adopters & 351 & 43.23 & 44 & 13.00 \\
Total & 812 & 100 & 331 & 100 \\
\hline
\end{tabular}

\section{Appendix 4: Type and definition of variables}

Table A5: Type and description of variables

\section{Variable type and description}

\section{Dependent variables}

Uptake

Dummy, equal to 1 for households that bought index-based insurance.

Credit rationing (1) Dummy, equal to 1 for credit-rationed households and 0 for creditunconstrained households

Credit rationing (2) Categorical, equal to 0 for credit-unconstrained, 1 for credit supplyrationed, and 2 for credit demand-constrained households

\section{Independent variables}

Risk aversion

Continuous, estimated as the value of constant relative risk aversion (CRRA) parameter based on the equation $u(\tau)=\frac{\tau^{1-C R R A}}{1-C R R A}$, where $u(\tau)$ is the payoff (utility) of the safe option, and $\tau$ is the payoff in the form of the expected value of a risky option. Payoffs were constructed from a Binswanger (1980) lottery game that the households played with small, real incentives in 2014.

Time preference Continuous, an exponential discount rate estimated as the value of $\delta$ from the equation $P V=F V\left(\frac{1}{1+\delta}\right)^{t}$, where $P V$ and $F V$ are present and future values, and $t$ is the discount period. Households were given a time preference game and chose from a fixed amount today $(P V)$ or a larger amount in the future $(F V)$.

Insurance product Dummy, equal to 1 for households that participated in a product promotion promotion meeting (campaign) of JICA and OIC and 0 for those that did not participate. In the expansion area (the control region), product promotion campaigns have taken place.

Peer influence

Dummy, equal to 1 for households that indicate that their peers, relatives, or neighbours who have bought insurance have convinced 
Basis risk

Gender

Intertemporal

adverse selection

Draft oxen

Education (years)

Distance from

market

Land size

Extension contact

Dependents

Dependency ratio

Population density

Housing quality

Livestock owned in

TLU

Access to non-farm employment

PSNP dependent

Net salvage value of physical assets (influenced) them to buy IBI, and 0 for others. In the expansion area, households indicated whether peer influence has convinced them to buy at the moment the insurance becomes available.

Continuous, distance of the household farm to the nearest metrological station measured in walking hours.

Dummy, equal to 1 if head of the household is male, 0 otherwise

Dummy, equals to 1 for households that indicate that, if insurance is available, they would postpone the uptake decision until the final insurance sales day, to make a better estimate of future weather conditions.

Number of draft oxen that the household uses for production

Household head's level of education in years of schooling

Distance from market in walking hours

Household's land holding, measured in a local unit called qarxi, where 1 qarxi $=0.25$ hectares

Dummy, equal to 1 for households that frequently make contact with extension agents; 0 for others

Number of dependents in a family

Dependents/family size

The ratio of the households' family size to land size.

Dummy, equals to 1 if the household owns an iron corrugated house

The size of livestock owned by the household measured in TLU-a standard unit of measurement.

Indicator variable with a one if a household has non-farm employment

Indicator variable with a one if the household is a beneficiary of a productive safety net program

the current value of the household's usable assets as estimated by the household 
Saving households monetary and non-monetary (cereal storage) saving in Birr

Wealth index An index constructed from information on various plant asset compositions of households, and the salvage values of each asset.

\section{Appendix 5: Robustness tests}

Tests whether the prediction of adopters in the control region are imprecise and biased

Table A6: Alternative determinants of uptake in treatment region

\begin{tabular}{|c|c|c|}
\hline Variables & (1) & (2) \\
\hline \multirow[t]{2}{*}{ Education (years) } & 0.017 & 0.025 \\
\hline & $(0.031)$ & $(0.031)$ \\
\hline \multirow[t]{2}{*}{ Family size } & $0.122^{* *}$ & $0.116^{* * *}$ \\
\hline & $(0.050)$ & $(0.039)$ \\
\hline \multirow[t]{2}{*}{ Coop member } & 0.300 & 0.252 \\
\hline & $(0.275)$ & $(0.330)$ \\
\hline \multirow[t]{2}{*}{ Draft oxen } & 0.100 & 0.103 \\
\hline & $(0.062)$ & $(0,055)$ \\
\hline \multirow[t]{2}{*}{ Peer influence } & $1.318 * * *$ & \\
\hline & $(0.401)$ & \\
\hline \multirow{2}{*}{ CRRA } & $-0.514 *$ & $-0.541 * *$ \\
\hline & $(0.274)$ & $(0.193)$ \\
\hline \multirow[t]{2}{*}{ Time preference } & 0.611 & $0.606^{* *}$ \\
\hline & $(0.438)$ & $(0.304)$ \\
\hline \multirow[t]{2}{*}{ Basis risk } & -0.039 & -0.016 \\
\hline & $(0.069)$ & $(0.041)$ \\
\hline \multirow[t]{2}{*}{ IBI product promotion } & $2.783 * * *$ & $3.002 * * *$ \\
\hline & $(0.340)$ & $(0.220)$ \\
\hline \multirow[t]{2}{*}{ Age } & 0.012 & 0.011 \\
\hline & $(0.010)$ & $(0.008)$ \\
\hline \multirow[t]{2}{*}{ Gender } & $-0.773 * *$ & $-0.731 * *$ \\
\hline & $(0.340)$ & $(0.294)$ \\
\hline \multirow[t]{2}{*}{ Intertemporal adverse selection } & $0.611^{* *}$ & $0.703 * * *$ \\
\hline & $(0.292)$ & $(0.190)$ \\
\hline \multirow[t]{2}{*}{ District 1} & -0.048 & -0.297 \\
\hline & $(1.085)$ & $(0.303)$ \\
\hline \multirow[t]{2}{*}{ District 2} & 0.016 & -0.158 \\
\hline & $(0.935)$ & $(0.237)$ \\
\hline \multirow[t]{2}{*}{ Constant } & $-3.640 * * *$ & $-2.696 * * *$ \\
\hline & $(1.151)$ & $(0.554)$ \\
\hline Pseudo R-squared & 0.33 & 0.31 \\
\hline Area under ROC curve & 0.86 & 0.85 \\
\hline Observations & 1,143 & 1,143 \\
\hline
\end{tabular}

Notes: Standard errors, in parentheses, were obtained by conducting a bootstrapping method on a system of equations, including the uptake equation and the credit-rationing equation. ${ }^{* * *} \mathrm{p}<0.01{ }^{* *} \mathrm{p}<0.05 .{ }^{*} \mathrm{p}<$ 0.1 . 
Table A7: Impacts of IBI uptake on credit rationing (alternative uptake equation)

\begin{tabular}{|c|c|c|c|c|}
\hline & (1) & (2) & (3) & (4) \\
\hline Variables & $\begin{array}{l}\text { Supply-side } \\
\text { credit } \\
\text { rationing }\end{array}$ & $\begin{array}{l}\text { Demand- } \\
\text { side credit } \\
\text { rationing }\end{array}$ & $\begin{array}{l}\text { Supply-side } \\
\text { credit } \\
\text { rationing }\end{array}$ & $\begin{array}{l}\text { Demand-side } \\
\text { credit } \\
\text { rationing }\end{array}$ \\
\hline \multirow[t]{2}{*}{ Uptake (W) } & $0.718^{*}$ & 0.387 & 0.527 & $1.240 * *$ \\
\hline & $(0.395)$ & $(0.500)$ & $(0.391)$ & $(0.562)$ \\
\hline \multirow[t]{2}{*}{ Region (R) } & $1.547 * * *$ & 0.343 & $1.472 * * *$ & 0.929 \\
\hline & $(0.369)$ & $(0.412)$ & $(0.385)$ & $(0.567)$ \\
\hline \multirow[t]{2}{*}{ Uptake*Region $\left(\mathrm{W}^{*} \mathrm{R}\right)$} & $-0.981 * *$ & -0.297 & $-0.808^{*}$ & -0.965 \\
\hline & $(0.4529)$ & $(0.527)$ & $(0.446)$ & $(0.609)$ \\
\hline \multirow[t]{2}{*}{ Age } & 0.002 & -0.004 & 0.002 & -0.004 \\
\hline & $(0.008)$ & $(0.008)$ & $(0.008)$ & $(0.008)$ \\
\hline \multirow[t]{2}{*}{ Education (years) } & 0.024 & -0.017 & 0.023 & -0.018 \\
\hline & $(0.024)$ & $(0.025)$ & $(0.024)$ & $(0.025)$ \\
\hline \multirow[t]{2}{*}{ Family size } & 0.011 & 0.005 & 0.0133 & -0.005 \\
\hline & $(0.078)$ & $(0.084)$ & $(0.078)$ & $(0.085)$ \\
\hline \multirow[t]{2}{*}{ Extension contract } & 0.841 & 0.347 & 0.851 & 0.274 \\
\hline & $(2.201)$ & $(0.748)$ & (2.192) & $(0.747)$ \\
\hline \multirow[t]{2}{*}{ District 1} & $-1.750 * * *$ & $1.763 * * *$ & $-1.737 * * *$ & $1.748 * * *$ \\
\hline & $(0.535)$ & $(0.442)$ & $(0.534)$ & $(0.441)$ \\
\hline \multirow[t]{2}{*}{ District 2} & $0.486 * *$ & $1.647 * * *$ & $0.483 * *$ & $1.642 * * *$ \\
\hline & $(0.220)$ & $(0.427)$ & $(0.219)$ & $(0.427)$ \\
\hline \multirow[t]{2}{*}{ Dependency ratio } & 0.011 & 0.159 & 0.074 & 0.235 \\
\hline & $(1.024)$ & (1.038) & (1.018) & (1.044) \\
\hline \multirow[t]{2}{*}{ Dependents } & -0.078 & -0.096 & -0.079 & -0.101 \\
\hline & $(0.152)$ & $(0.161)$ & $(0.151)$ & $(0.162)$ \\
\hline \multirow[t]{2}{*}{ Coop member } & -0.374 & -0.650 & -0.345 & -0.706 \\
\hline & $(0.340)$ & $(0.421)$ & $(0.315)$ & $(0.420)$ \\
\hline \multirow[t]{2}{*}{ Constant } & -3.167 & -2.950 & -3.141 & $-3.529 * * *$ \\
\hline & $(2.275)$ & (2.934) & $(2.265)$ & (1.124) \\
\hline Observations & 1,143 & 1,143 & 1,143 & 1,143 \\
\hline
\end{tabular}

Notes: Standard errors, in parentheses, are based on a bootstrap method, where the bootstrap is performed for a system of equations, containing the uptake equation (Estimation (1) in Table A6 for estimation (1) and (2) in Table A7; and estimation (2) in Table A6, for estimations (3) and (4) in Table A7) and the supply and demand-side credit rationing equations. The demand-side and supply-side credit rationing equations follow from a multinomial logit regression. The bootstrap relies on 2000 repetitions. ${ }^{* *} \mathrm{p}<0.01 .{ }^{* *} \mathrm{p}<0.05$. ${ }^{*} \mathrm{p}$ $<0.1$. 
Table A8: IBI uptake and credit rationing (cut-off value based on highest Youden Index)

\begin{tabular}{|c|c|c|}
\hline Variables & $\begin{array}{c}\text { Uptake (1) } \\
\text { Supply-side credit rationing }\end{array}$ & $\begin{array}{c}\text { Uptake (2) } \\
\text { Demand-side credit rationing }\end{array}$ \\
\hline \multirow[t]{2}{*}{ Uptake (W) } & $0.933^{* *}$ & -0.351 \\
\hline & $(0.375)$ & $(0.465)$ \\
\hline \multirow[t]{2}{*}{ Region (R) } & $1.584 * * *$ & 0.032 \\
\hline & $(0.310)$ & $(0.295)$ \\
\hline \multirow[t]{2}{*}{ Uptake*Region ( W*R) } & $-1.319 * * *$ & 0.397 \\
\hline & $(0.443)$ & $(0.491)$ \\
\hline \multirow[t]{2}{*}{ Age } & 0.002 & -0.004 \\
\hline & $(0.008)$ & $(0.008)$ \\
\hline \multirow[t]{2}{*}{ Education (years) } & 0.024 & -0.018 \\
\hline & $(0.024)$ & $(0.024)$ \\
\hline \multirow[t]{2}{*}{ Family size } & 0.0226 & $-9.68^{\mathrm{e}}-06$ \\
\hline & $(0.075)$ & $(0.085)$ \\
\hline \multirow[t]{2}{*}{ Extension contract } & 0.930 & 0.380 \\
\hline & $(1.989)$ & $(0.599)$ \\
\hline \multirow[t]{2}{*}{ District 1} & $-1.772 * * *$ & $1.773 * * *$ \\
\hline & $(0.432)$ & $(0.450)$ \\
\hline \multirow[t]{2}{*}{ District 2} & $0.480 * *$ & $1.655 * * *$ \\
\hline & $(0.210)$ & $(0.437)$ \\
\hline \multirow[t]{2}{*}{ Dependency ratio } & 0.176 & -0.029 \\
\hline & $(0.974)$ & $(1.071)$ \\
\hline \multirow[t]{2}{*}{ Dependents } & -0.097 & -0.070 \\
\hline & $(0.144)$ & $(0.165)$ \\
\hline \multirow[t]{2}{*}{ Cooperative member } & -0.366 & -0.586 \\
\hline & $(0.307)$ & $(0.537)$ \\
\hline \multirow[t]{2}{*}{ Constant } & -3.353 & $-2.603 * * *$ \\
\hline & $(2.056)$ & $(0.904)$ \\
\hline Observations & 812 & 812 \\
\hline Youden index & 0.61 & 0.61 \\
\hline Cut-off value & 0.666 & 0.666 \\
\hline Correctly classified & $81 \%$ & $81 \%$ \\
\hline Sensitivity & $82 \%$ & $82 \%$ \\
\hline Specificity & $79 \%$ & $79 \%$ \\
\hline
\end{tabular}

Note: Standard errors, in parentheses, are based on a bootstrap method, where the bootstrap is performed for a system of equations, containing the uptake equation (Estimation (1) in Table 4.5) and the supply and demand-side credit rationing equations. The demand-side and supply-side credit rationing equations follow from a multinomial logit regression. ${ }^{* * *} \mathrm{p}<0.01 .{ }^{* *} \mathrm{p}<0.05 . * \mathrm{p}<0.1$. Correctly classified; Sensitivity and Specificity refer to values for the "eligible" kebeles. 


\section{Tests whether the credit-rationing equation is wrongly specified}

Table A9: IBI uptake and credit rationing (alternative controls in credit rationing equation)

\begin{tabular}{|c|c|c|c|c|}
\hline Variables & $\begin{array}{c}\text { (1) } \\
\text { Supply-side } \\
\text { credit rationing }\end{array}$ & $\begin{array}{c}(2) \\
\text { Demand-side } \\
\text { credit rationing }\end{array}$ & $\begin{array}{l}\text { (3) } \\
\text { Supply-side credit } \\
\text { rationing }\end{array}$ & $\begin{array}{c}\text { (4) } \\
\text { Demand-side credit } \\
\text { rationing }\end{array}$ \\
\hline Uptake (W) & $\begin{array}{c}0.779 * * \\
(0.384)\end{array}$ & $\begin{array}{c}0.628 \\
(0.585)\end{array}$ & $\begin{array}{c}0.472 \\
(1.207)\end{array}$ & $\begin{array}{c}0.276 \\
(0.978)\end{array}$ \\
\hline Region (R) & $\begin{array}{l}1.117 * * * \\
(0.343)\end{array}$ & $\begin{array}{c}0.440 \\
(0.448)\end{array}$ & $\begin{array}{l}1.508 * * * \\
(0.411)\end{array}$ & $\begin{array}{l}0.844 * \\
(0.444)\end{array}$ \\
\hline Uptake*Region $\left(\mathrm{W}^{*} \mathrm{R}\right)$ & $\begin{array}{c}-0.938 * * \\
(0.428)\end{array}$ & $\begin{array}{l}-0.720 \\
(0.594)\end{array}$ & $\begin{array}{c}-1.039 * * \\
(0.461)\end{array}$ & $\begin{array}{l}-0.7433 \\
(0.536)\end{array}$ \\
\hline Age & & & $\begin{array}{l}-0.001 \\
(0.008)\end{array}$ & $\begin{array}{l}-0.003 \\
(0.008)\end{array}$ \\
\hline Family size & & & $\begin{array}{c}0.020 \\
(0.079)\end{array}$ & $\begin{array}{l}-0.005 \\
(0.088)\end{array}$ \\
\hline District 1 & & & $\begin{array}{c}-1.869 * * * \\
(0.543)\end{array}$ & $\begin{array}{l}1.750 * * * \\
(0.488)\end{array}$ \\
\hline District 2 & & & $\begin{array}{c}0.505 \\
(0.226)\end{array}$ & $\begin{array}{l}1.623 * * * \\
(0.477)\end{array}$ \\
\hline Extension contract & & & $\begin{array}{c}0.839 \\
(2.200)\end{array}$ & $\begin{array}{c}0.247 \\
(0.738)\end{array}$ \\
\hline Dependency ratio & & & $\begin{array}{c}0.345 \\
(1.071)\end{array}$ & $\begin{array}{l}-0.057 \\
(1.061)\end{array}$ \\
\hline Dependents & & & $\begin{array}{l}-0.122 \\
(0.155)\end{array}$ & $\begin{array}{l}-0.075 \\
(0.168)\end{array}$ \\
\hline Coop member & & & $\begin{array}{l}-0.413 \\
(0.325)\end{array}$ & $\begin{array}{l}-0.717 \\
(0.445)\end{array}$ \\
\hline Peer influence & & & $\begin{array}{c}0.400 \\
(0.265)\end{array}$ & $\begin{array}{l}-0.265 \\
(0.324)\end{array}$ \\
\hline Risk aversion & & & $\begin{array}{l}-0.063 \\
(0.181)\end{array}$ & $\begin{array}{l}0.414 * * \\
(0.206)\end{array}$ \\
\hline Time preference & & & $\begin{array}{l}0.567 * * \\
(0.259)\end{array}$ & $\begin{array}{c}0.127 \\
(0.298)\end{array}$ \\
\hline Basis risk & & & $\begin{array}{l}-0.021 \\
(0.041)\end{array}$ & $\begin{array}{c}0.002 \\
(0.059)\end{array}$ \\
\hline IBI product promotion & & & $\begin{array}{c}0.240 \\
(1.173)\end{array}$ & $\begin{array}{c}0.860 \\
(0.687)\end{array}$ \\
\hline Gender & & & $\begin{array}{l}0.752 * * \\
(0.274)\end{array}$ & $\begin{array}{l}-0.099 \\
(0.235)\end{array}$ \\
\hline IAS & & & $\begin{array}{l}-0.213 \\
(0.175)\end{array}$ & $\begin{array}{c}0.232 \\
(0.184)\end{array}$ \\
\hline Constant & $\begin{array}{c}-1.986 * * * \\
(0.305)\end{array}$ & $\begin{array}{c}-1.629 * * \\
(0.444)\end{array}$ & $\begin{array}{l}-3.871 * \\
(2.284)\end{array}$ & $\begin{array}{c}-3.663 * * * \\
(1.130)\end{array}$ \\
\hline Observations & 1,143 & 1,143 & 1,143 & 1,143 \\
\hline
\end{tabular}

Note: IAS refers to Intertemporal Adverse Selection. Standard errors, in parentheses, are based on a bootstrap method, where the bootstrap (2000 repetitions) is performed for a system of equations, containing the uptake equation (Equation 1 in Table 4.5) and the supply and demand-side credit rationing equations. The demand-side and supply-side credit rationing equations follow from a multinomial logit regression. $* * * \mathrm{p}<0.01 .{ }^{* *} \mathrm{p}<0.05 . * \mathrm{p}<0.1$. 
Tests whether the estimates suffer from endogeneity bias and reverse causality

Table A10: Adding credit rationing dummy to uptake equation

Variables

Age

Uptake of IBI

District 1

$(0.007)$

District 1

$-0.349$

$(0.269)$

District 2

$-0.141$

(0.235)

Peer influence

$1.294 * * *$

$(0.240)$

Risk aversion

$-0.545^{* * *}$

Time preference

$(0.195)$

0.578

(0.311)

Basis risk

$-0.032$

(0.040)

IBI product promotion

$2.888 * * *$

Gender

(0.220)

$-0.552$

(0.283)

Intertemporal adverse selection

$0.604 * * *$

Credit rationed (dummy)

$(0.191)$

0.048

(0.190)

Constant

$-2.916^{* * *}$

(0.958)

Area under ROC

0.85

Correctly classified

$79.7 \%$

Pseudo $\mathrm{R}^{2}$

0.32

Observations

812

Notes: Standard errors, in parentheses (with "kebele" as the cluster variable). ${ }^{* * *} \mathrm{p}<0.01 .{ }^{* *} \mathrm{p}<0.05 .{ }^{*} \mathrm{p}$ $<0.1$. 
Table A11: Impacts of IBI uptake on credit rationing (Credit rationing in uptake equation)

\begin{tabular}{|c|c|c|}
\hline Variables & $\begin{array}{l}\text { Supply-side Credit } \\
\text { rationing }\end{array}$ & $\begin{array}{c}\text { Demand-side credit } \\
\text { rationing }\end{array}$ \\
\hline \multirow[t]{2}{*}{ Region (R) } & $1.615^{* * *}$ & 0.539 \\
\hline & $(0.358)$ & $(0.503)$ \\
\hline \multirow[t]{2}{*}{ Uptake (W) } & $0.853 * * *$ & 0.722 \\
\hline & $(0.396)$ & $(0.671)$ \\
\hline \multirow[t]{2}{*}{ Region*Uptake (W*R) } & $-1.109 * * *$ & -0.617 \\
\hline & $(0.434)$ & $(0.647)$ \\
\hline \multirow[t]{2}{*}{ Age } & 0.002 & -0.005 \\
\hline & $(0.008)$ & $(0.008)$ \\
\hline \multirow[t]{2}{*}{ Education (years) } & 0.025 & -0.014 \\
\hline & $(0.024)$ & $(0.025)$ \\
\hline \multirow[t]{2}{*}{ Family size } & 0.014 & 0.013 \\
\hline & $(0.076)$ & $(0.085)$ \\
\hline \multirow[t]{2}{*}{ Extension contact } & 0.837 & 0.318 \\
\hline & $(1.970)$ & $(0.600)$ \\
\hline \multirow[t]{2}{*}{ District 1} & $-1.753 * * *$ & $1.772 * * *$ \\
\hline & $(0.429)$ & $(0.450)$ \\
\hline \multirow[t]{2}{*}{ District 2} & $0.481 * *$ & $1.650 * * *$ \\
\hline & $(0.209)$ & $(0.438)$ \\
\hline \multirow[t]{2}{*}{ Dependency ratio } & 0.040 & 0.246 \\
\hline & $(0.978)$ & $(1.067)$ \\
\hline \multirow[t]{2}{*}{ Dependents } & -0.084 & -0.112 \\
\hline & $(0.146)$ & $(0.164)$ \\
\hline \multirow[t]{2}{*}{ Coop member } & -0.396 & -0.684 \\
\hline & $(0.304)$ & $(0.537)$ \\
\hline \multirow[t]{2}{*}{ Constant } & -3.233 & $-3.148 * * *$ \\
\hline & $(2.042)$ & $(0.993)$ \\
\hline Observations & 1,143 & 1,143 \\
\hline
\end{tabular}

Note: Standard errors, in parentheses, are based on a bootstrap method, where the bootstrap is performed for a system of equations, containing the uptake equation (Estimation (1) in Table A10) and the supply and demand-side credit rationing equations. The demand-side and supply-side credit rationing equations follow from a multinomial logit regression. The bootstrap relies on 2000 repetitions. ${ }^{* * *} p<0.01 .{ }^{* *} p<0.05$. ${ }^{*} p$ $<0.1$. 


\section{Tests whether the standard errors of the estimates are biased due to clustering effects}

Table A12: Impacts of IBI uptake on credit rationing (with cluster robust standard errors)

\begin{tabular}{|c|c|c|c|c|}
\hline Variables & $\begin{array}{l}\text { (1) } \\
\text { Supply-side } \\
\text { credit } \\
\text { rationing }\end{array}$ & $\begin{array}{l}\text { (2) } \\
\text { Demand- } \\
\text { side credit } \\
\text { rationing }\end{array}$ & $\begin{array}{l}\text { (3) } \\
\text { Supply- } \\
\text { side credit } \\
\text { rationing }\end{array}$ & $\begin{array}{l}\text { (4) } \\
\text { Demand-side } \\
\text { credit } \\
\text { rationing }\end{array}$ \\
\hline Region (R) & $\begin{array}{c}1.615 * * * \\
(0.389)\end{array}$ & $\begin{array}{c}0.539 \\
(0.794)\end{array}$ & $\begin{array}{c}1.615 * * * \\
(0.445)\end{array}$ & $\begin{array}{c}0.539 \\
(0.522)\end{array}$ \\
\hline Uptake (W) & $\begin{array}{c}0.852 * * * \\
(0.287)\end{array}$ & $\begin{array}{c}0.722 \\
(0.896)\end{array}$ & $\begin{array}{l}0.852 * \\
(0.483)\end{array}$ & $\begin{array}{c}0.722 \\
(0.669)\end{array}$ \\
\hline Region*Uptake $\left(\mathrm{W}^{*} \mathrm{R}\right)$ & $\begin{array}{c}-1.109 * * \\
(0.421)\end{array}$ & $\begin{array}{l}-0.617 \\
(0.918)\end{array}$ & $\begin{array}{c}-1.109 * * \\
(0.567)\end{array}$ & $\begin{array}{l}-0.617 \\
(0.646)\end{array}$ \\
\hline Constant & $\begin{array}{l}-3.233 \\
(1.050)\end{array}$ & $\begin{array}{l}-3.148 \\
(2.594)\end{array}$ & $\begin{array}{l}-3.233 \\
(1.861)\end{array}$ & $\begin{array}{l}-3.148 \\
(2.130)\end{array}$ \\
\hline Observations & 1,143 & 1,143 & 1,143 & 1,143 \\
\hline
\end{tabular}

Notes: Standard errors, in parentheses, are based on a cluster bootstrap method (kebeles as cluster variable, for estimations (1) and (2); Iddir as cluster variable for estimations (3) and (4), where the bootstrap is performed for a system of equations, containing the uptake equation (Estimation (1) in Table 4.5) and the supply and demand-side credit rationing equations. The demand-side and supply-side credit rationing equations follow from a multinomial logit regression. The cluster bootstrap relies on 1000 repetitions. The equations include same set of controls as in base model (see Table 4.9), which are not presented for reasons of space. $* * * \mathrm{p}<0.01 . * * \mathrm{p}<0.05{ }^{*} \mathrm{p}<0.1$.

\section{Tests whether there are remaining program placement effects}

Table A13: IBI uptake and credit rationing (kebele fixed effects)

(1) (2)

\begin{tabular}{lcc} 
Variables & Supply-side credit rationing & Demand-side credit rationing \\
\hline Uptake $(\mathrm{W})$ & $0.780^{* *}$ & 0.629 \\
& $(0.379)$ & $(0.587)$ \\
Region $(\mathrm{R})$ & 3.239 & 1.932 \\
& $(3.652)$ & $(4.008)$ \\
Uptake*Region $(\mathrm{W} * \mathrm{R})$ & $-1.279^{* * *}$ & -0.676 \\
& $(0.439)$ & $(0.616)$ \\
Constant & $-1.725^{* * *}$ & $-1.389^{* * *}$ \\
& $(0.345)$ & $(0.487)$
\end{tabular}

Observations $1,143 \quad 1,143$

Notes: For estimations (1) and (2), standard bootstrap standard errors are presented in parentheses. The bootstrap is performed for a system of equations, containing the uptake equation (Estimations (1) in Table $4.5)$ and the supply and demand-side credit rationing equations. The demand-side and supply-side credit rationing equations follow from a multinomial logit regression. The estimations also include kebele fixed effects. $* * * \mathrm{p}<0.01 . * * \mathrm{p}<0.05 . * \mathrm{p}<0.1$. 


\title{
Chapter 5
}

\section{Does index-based insurance improve household welfare? Empirical evidence based on panel data in south-eastern Ethiopia}

\begin{abstract}
Evidence on the welfare impacts of index-based insurance is scant. This study examines the impact of sustained uptake of index-based insurance on household consumption and investment in inputs. A two-round panel data were collected from smallholders in Ethiopia, and the difference-in-difference estimator was employed to reduce program placement and self-selection biases, arising from time-invariant unobserved heterogeneity. Results indicate that adoption of index insurance has causally increased household consumption and investment in high-risk high-return agricultural inputs. Further, accounting for the intensity of adoption, results suggest that repeated adoption of index insurance has cumulative lasting effect on household welfare.
\end{abstract}

Keywords: Index insurance; Welfare impacts; Ethiopia; Smallholders

This chapter is based on:

Belissa (2018): Does Index-based insurance improve household welfare? Empirical evidence based on panel data in south-eastern Ethiopia. Working paper 


\subsection{Introduction}

Increased interest is seen in recent years in studying index-based insurance (IBI) as a potential drought insurance technology that constitutes a pro-poor climate risk management strategy. However, evidence on the welfare impacts of this innovation is scant. In the absence of data on repeated observations, identification and measurement problems create the difficulty to assess the impact of an intervention implemented in a natural experimental setting. Impact estimations that rely on simple comparison of outcomes of participants with that of non-participants often suffer from selection biases. In technology adoption, one form of such bias is self-selection bias that arises from the tendency of adopters to engage in adoption decisions being motivated by their own unique characteristics like individualspecific entrepreneurial spirit. Self-selection bias results in biased impact estimates since unmeasured individual attributes of adopters may simultaneously affect participation in adoption and the outcome of adoption. Program placement bias that occurs when interventions are undertaken on non-random basis is another form of selection bias (Ravallion 2007). In IBI intervention, insurance firms may choose villages for implementation based upon characteristics that may not be observable to the researcher.

Randomized experimental designs that create exogenous variations in sub-groups of treated and controlled units may help to overcome the problems of selection biases. However, randomization alone is not a necessary and sufficient condition to capture the complete impact of an intervention. Karlan and Goldberg (2007) explain that since interventions often take long time to establish lasting effects, impact estimates based on one period of experimental or quasi-experimental data meant to randomize over potential sources of selection may not reflect the full effect of an intervention. The timing and duration of exposures is important in assessing the impact of interventions (Ravallion 2001; Ahlin and Jiang 2008; Tedeschi 2008; King and Behrman 2009; Berhane and Gardebroek 2011).

Various studies (Pitt and Khandker 1998; Coleman 1999) depend on cross-sectional data analysis techniques, and exploit program-specific designs or innovative quasi-experimental survey methods to generate control and treatment groups. Estimation techniques like Heckman correction and instrumental variable (IV) are also used to control for selection biases stemming from unobserved heterogeneity. However, these procedures still impose distributional and functional form assumptions. Furthermore, identifying a valid instrument that determines the treatment status but not the outcome variable remains an empirical 
challenge in IV regressions (Staiger and Stock 1997). The IV approach also ignores interactions between the treatment variable and other covariates as it assumes that the treatment variable has only a parallel shift effect (intercept effect but not slope effect) with respect to the outcome variable (Wooldridge 2002). Hence, estimating unbiased impact of an intervention that may accrue overtime requires dealing with individual, village or intervention-specific time-invariant heterogeneity, using for instance, a panel dataset. The difference-in-difference approach is a better alternative to control for time-invariant unobservable heterogeneity that may cofound with impacts (Copestake et al. 2001; Khandker 2005; Coleman 2006; Tedeschi 2008). This study investigates whether adoption of IBI can provide for welfare enhancements at household level using this method. We use a two-round panel data on two welfare indicators: household consumption and investment risk-taking behaviour. We measure household consumption in terms of per capita weekly food consumption, annual non-food and total consumption expenditures. Similarly, we measure investment risk-taking behaviour of the households in terms of their investment in high-risk high-return inputs including investment in chemical fertilizer, improved seed variety and pesticides/herbicides. First, we estimate the impact of participation at the extensive margin of IBI adoption using the difference-in-difference method. Then, we estimate the impacts of the intensity of IBI adoption at the intensive margin using a flexible specification that takes adoption cycles or years into account. Both ways show that adoption of IBI has significant welfare improvement effects in terms of increased consumption and investment in high-risk high-return agricultural inputs at household level. The impact of IBI adoption on consumption and investment in high-risk high-return inputs also increases with the frequency of IBI adoption.

The remaining sections of this chapter are organized as follows. Section 5.2 presents review studies on the impacts of IBI adoption. Section 5.3 details the study context, IBI implementation in the study area and the design of the survey. Section 5.4 explains the impact identification strategy employed. Section 5.5 presents the results. Section 5.6 concludes the chapter.

\subsection{Review of microinsurance impact studies}

Barnett et al. (2008) explain that the economic returns to adoption of IBIs are potentially broad and substantial, ranging from inducing households to make more prudential investments, providing better management for consumption risk, crowding-in finance for 
ancillary investment and enhancing local adaptation to climate change. Corroborating this, various studies show that where effectively implemented, IBIs have welfare improvement impacts. For instance, Karlan et al. (2014) identified that lack of access to insurance is the limiting factor to investment for maize farmers in Ghana. The study revealed that smallholders who purchased IBI have a 13\% more investment in agricultural inputs than others. Fuchs and Wolff (2011) assessed the impact of insurance against catastrophic drought on corn yields, area cultivated in corn, and per capita income and expenditures of smallholders in Mexico. Results indicate that where coverage is available corn yield was increased by $8 \%$, with gains in income and expenditures. These evidences indicate that adoption of insurance induces ex-ante risk management responses. Mobarak and Rosenzweig (2013) used a randomized experiment where IBI is offered to Indian cultivators, finding that IBI helps cultivators reduce self-insurance and switch to riskier, higher-yield production techniques. Similarly, in Cai et al. (2015) it was found that insurance for sows significantly increased farmers' tendency to raise sows in south-western China, where sow production is a risky production activity with potentially large returns. In another experiment, Cai (2016) demonstrates that weather insurance induces tobacco farmers in China to increase the land devoted to this risky crop by $20 \%$. This finding implies reduced diversification among tobacco farmers, consistent with less self-insurance. Using an experimental game method, Vargas-Hill and Viceisza (2013) indicated that insurance induces farmers in rural Ethiopia to take greater, yet profitable risks, by increasing (theoretical) purchase of chemical fertilizer. An important inference from all these evidences is that adoption of IBI can enhance prudential investment risk-taking behaviour among farm households in developing counties.

IBIs were also evidenced to have impacts on ex-post shock coping. In an experimental game conducted in China, Cheng (2014) studies the effect of offering IBI to risk-rationed households. The study reports that more than half of the risk-rationed farmers decided to apply for credit when IBI is available to them. Similarly, Giné and Yang (2009) studied an intervention where maize farmers in Malawi were offered with a choice between a loan and a loan plus insurance. The study found, however, that demand for the basic loan was $13 \%$ higher than that for the insured loan. In Carter et al. (2016), it was argued that a stand-alone insurance product does not provide additional benefits to farmers who have low collateral. The reason is that, if no formal insurance is available, only farmers with high collateral may choose not to borrow, because they do not want to put their collateral at risk. Cai (2016) also finds that insurance causes households to decrease savings by more than $30 \%$, pointing that 
households were building up extra precautionary savings in order to better smooth consumption in the case of a shock. Belissa et al. (2018c) examined the impact of IBI on credit rationing in Ethiopia, and found that insurance coverage is positively related to credit use. The study then goes on to examine if the change is associated with reduced demand side or supply-side rationing, finding that the changes are due to differences in supply-side credit rationing.

Adoptions of IBIs were also evidenced to reinforce the functioning of the extant social insurance mechanisms. Mobarak and Rosenzweig (2012) show that, in rural India, the existence of informal risk-sharing networks among members of a sub-caste increases demand for IBI when informal risk-sharing covers idiosyncratic losses. Belissa et al. (2018b) examined whether uptake of IBI can be enhanced by postponing the premium payment towards shortly after harvest, and by marketing the product through the extant social insurance institutions known as Iddirs in Ethiopia. The study found that coaxing the marketbased IBI with the predominant social insurance increases uptake, as compared with selling such insurance through conventional statutory channels like state-owned cooperatives. On the other hand, Fuchs and Rodriguez-Chamussy (2011) analysed the impact of insurance payouts on voter behaviour in the 2006 presidential election in Mexico. Using a regression discontinuity design with electoral section as a unit of analysis, the study questions whether insurance payouts received by farmers in the electoral section in 2005 have affected voting behaviour in favour of the incumbent political party in the subsequent 2006 election. The study finds that disaster relief buys votes. The incumbent party is estimated to have gained $8 \%$ more votes where payouts had been made prior to the election.

However, though insurance is usually targeted to smooth consumption or enhance risk-taking behaviour (Townsend et al. 1994:1995), evidences in this regard are limited. Janzen and Carter (2013) explained that access to an index-based livestock insurance (IBLI) in northern Kenya helped pastoral households to smooth their asset and consumption that constitute the two key dimensions of self-insurance. The study revealed that after the intervention, poor pastoral households are less likely to destabilize their consumption in response to drought, while those who are better off are less likely to have to compromise their accumulated assets. Insured households are observed to be less dependent on food aid and other forms of assistance, which indicates their better ability to cope with shocks. The impact of the IBLI intervention was also selective based on wealth position of the pastoral households. Studies also show that adoption of IBI enhances households' access to other financial markets. The 
current study provides evidences on the impact of repeated adoption of index insurance for crops on household welfare from the perspectives of consumption and production (i.e., risking taking from investment in high-risk high-return production inputs).

\subsection{Study context, IBI intervention, survey design and data}

\subsubsection{Context}

This study is undertaken in the central Rift Valley zone of the Oromia regional state in southeastern Ethiopia. The Rift Valley zone is a semi-arid plain plateau area with a low-land agroecology. The pattern and intensity of rainfall exhibits considerable spatial and temporal variation, with a bimodal type of distribution. The area receives very low level of annual average rainfall. Rainfall seasons are from May to August and during October and November. Moisture stress and drought frequently causes devastating crop failure, rampant livestock mortality and herd collapse. Major droughts in the area include the 2015-16 drought which followed the historical trend of droughts during 1973-74, 1983-84, 1991-92, 1999-2000, 2005-06 and 2011-12 (Dercon 2004). Households in the area are smallholder subsistence farmers who mainly produce maize and wheat. They often face drought-induced income shocks that translate into erratic consumption patterns. Their ex-post shock coping mechanisms include reducing frequency of meals per day, distress livestock sells, reducing farm investment on chemical fertilizer and improved seed varieties, forcing pupils to withdraw from school for casual labour, renting land and family labour for local landlords and wage employment on floriculture farms of foreign investors. Future drought shock predictions in Ethiopia are pessimistic with expected rise in temperature from 23.08 to $26.92^{\circ} \mathrm{C}$ (Hulme et al. 2001; Meze-Hausken 2009). As a result, the wide crop-livestock mixed farming system in arid and semi-arid areas like the Rift Valley zone were projected to transform into extensive systems to respond to the risks of climate change (Meinke and Stone 2005; Thornton et al. 2010). Hence, innovative drought risk management mechanisms like adoption of drought insurances were highly required for farm households in the area. A large proportion of the smallholders in the study area have no access to formal financial service. They also do not have access to non-traditional risk coping mechanisms ${ }^{45}$.

\footnotetext{
${ }^{45}$ However, the functioning of indigenous social institutions is actively remarkable. Burial societies (Iddirs) provide social insurance services when households lose their bread winners like death of household heads or draft oxen.
} 


\subsubsection{IBI in the study area}

To improve the resilience of households in the face of climate change, Japan International Cooperation Agency (JICA) and Oromia Insurance Company (OIC) jointly initiated the implementation of IBI for crops in the Rift Valley zone of Ethiopia in 2013. The IBI scheme was implemented in five districts including Boset, Bora, Ilfata, Adamitullu-Jido-Kombolcha (AJK) and Arsi Negele. Before the initial intervention in 2013, OIC and JICA that provides the financial support and Ethiopian Ministry of Agriculture that provides the technical support for the intervention have discussed and identified districts in which drought shocks are common in Ethiopia. Most of these districts are located in the Rift Valley zone. The partners then held a focus group discussion (FGD) with selective representative farmers from each kebele within each of the selected districts. Based on this discussion, many kebeles which have severe drought experience in the past were identified. However, then, it was found that the financial support that JICA allotted for the 2013 weather index insurance intervention was not adequate to cover all the identified drought-prone kebeles. Therefore, the partners randomly considered some kebeles for the first intervention in 2013 and rankfiled the remaining kebeles to be considered in subsequent interventions. IBI is often marketed and sold twice per year during April and during September, months preceding the two rainy seasons, to provide coverage against losses during the seedling and flowering stages of crop growth, respectively. Major targeted stable food crops to be insured include maize, wheat, barley and teff. However, payout is not crop-specific. OIC currently uses vegetation index crop insurance (VICI). VICI is a modified product with better quality, designed based on the intensity of vegetation cover or greenery on the earth's surface. Greenery level is measured by a satellite indicator known as normalized difference vegetation index (NDVI). In VICI design, NDVI is extracted at a geospatial resolution of $1 \mathrm{~km}^{2}$. The current VICI product used by OIC is based on average NDVI of 16 years. NDVI reflects the already accumulated result of rain on crop growth. It is a primary measurement with no assumptions or calibrations. It is the proven standard index, in use by all early warning units globally ${ }^{46}$.

\footnotetext{
${ }^{46}$ NDVI is measured through images obtained from a geo-satellite weather infrastructure known as GeoNetCast System. The system reads to see if the observed area contains live green vegetation or not. The data from these images are converted into digital numbers (DN-values), i.e. Integers from 0 to 255 creating the NDVI. In order to deal with transaction costs, it divides the geographical coverage into CPS zones. NDVI is computed for each zone at grids of $1 \mathrm{~km}^{2}$. Payout is calculated for a decal or
} 


\subsubsection{Survey design and data}

Data used in this study were collected from smallholders in the Rift Valley zone in southeastern Ethiopian. A two-round survey conducted with two-year intervals during 2015 and 2017 were considered in this study. Recruitment of the sample households was worked out as follows. First, we selected three districts, namely Bora, AJK and Arsi Negele, out of the five districts where OIC implemented IBI. Second, we identified a random sample of kebeles within the three districts, including those kebeles covered by IBI as well as those that OIC did not cover. Finally, sample households were randomly drawn from all these selected kebeles.

In the first round of survey that we conducted during January-April 2015, data were collected from a total of 1143 households, out of which 461 were adopters and 682 were non-adopters of IBIs. However, during the second round survey, we observed that adoption status was changed since some new households joined and others dropped out the adoption. We excluded these two sets of households from our analysis. Hence, we used a balanced dataset of only 149 persistent adopters and 543 never-adopters. This enables us to identify the impact of sustained uptake of IBI over the period 2015-2017. Information on household, village and IBI intervention characteristics as well as investment in agricultural inputs, consumption, use of financial services as well as village infrastructure and access to markets were collected. In addition, we collected data on uptake, the number of years of adoption per household and payout from OIC.

The impact of IBI adoption was measured for two welfare indicators: household consumption and investment in high-risk high-return inputs. Both set of variables are continuous in nature. Household consumption is an aggregate of selected food and non-food consumption. Food items consumed both from own sources and from purchases over a period of one week were included ${ }^{47}$.

every 10 days period. Hence, trigger and exit thresholds are computed for CPS zones and 10-day period specific. The insurance coverage period is crop growth season specific. Payout is not cropspecific, but $1 \mathrm{~km}^{2}$ grid (location) specific.

${ }^{47}$ Food items include food grains, fruits, vegetables, milk and milk products, beef, meat and meat products, cooking oil, salt, and coffee, tea, and other leisure drinks. Non-food items include clothing and footwear, gas and fuel, schooling, health, family events, and household durables. Note that the recall period for estimating food items consumed was 7 days, and for non-food items like cloths was a year. 


\subsection{Identification strategy}

We analyze the effects of sustained uptake of IBI on household welfare using the difference-in-difference method in two different ways. First, we measure the effect of adoption at the extensive margin as follows:

$$
\mathrm{W}_{\mathrm{hkjt}}=\beta_{0}+\beta_{1} \mathrm{IBI}_{\mathrm{h}}+\beta_{2} \text { Post }_{\mathrm{t}}+\beta_{3}\left(\mathrm{IBI} * \text { Post }_{\mathrm{ht}}+\beta_{4} \mathrm{R}_{\mathrm{k}}+\beta_{5} \mathrm{X}_{\mathrm{h}}+\beta_{6} \text { Strata }_{\mathrm{j}}+\varepsilon_{\mathrm{hkjt}}\right.
$$

where $W_{h k j t}$ is the welfare outcome of interest for household $h$ in region $k$ and district $j$ at time $t ; I B I_{h}$ is an indicator for sustained uptake of IBI that equals ' 1 ' if household $h$ is an adopter of IBI in both 2015 and 2017 but equals ' 0 ' if the household is non-adopter; Post $_{t}$ is an indicator that equals ' 1 ' if the observation is in 2017 and equals ' 0 ' if the observation is in 2015; $R_{k}$ is an indicator that equals ' 1 ' if the household is in the initial adoption region and ' 0 ' if the household is in the expansion (subsequent phase adoption) region; $X_{h}$ is a vector of covariates including households' demographic characteristics, assets and access to services as described in Appendix 6; Strata ${ }_{j}$ represents the three districts (one of which is excluded from the regression); and $\varepsilon_{h k j t}$ is the error term. Second, following Berhane and Gardebroek (2011), we measure the effect of intensity of adoption of IBI at the intensive margin as follows:

$$
W_{\text {hkjt }}=\beta_{0}+\beta_{1} P_{1}+\beta_{2} P_{2}+\beta_{3} P_{3}+\beta_{4} P_{4}+\beta_{5} R_{k}+\beta_{6} X_{h}+\beta_{7} \text { Strata }_{j}+\varepsilon_{\text {hkjt }}
$$

where $P_{1} \ldots P_{4}$ are dummies indicating one-year, two-years, three-years and four-years adoption of IBI respectively, and the other variables are as defined in eq. (5.1). Hence, in eq. (5.2), intensities of IBI adoption are represented by dummy variables for each of the number of years that the households were stayed in adoption phase.

\subsection{Results}

\subsubsection{Characteristics of the sample households}

Summary statistics on the characteristics of IBI-adopter and non-adopter households is presented in Table 5.1. IBI-adopter and non-adopter households had statistically insignificant differences in terms of their various demographic characteristics including age, gender of the household head and dependency ratio. However, compared to non-adopters, IBI-adopters were more educated and had larger family size. In terms of production assets, IBI-adopters had larger land and livestock sizes compared to non-adopter households. 
Table 5.1: Summary statistics of IBI adopter and non-adopter households

\begin{tabular}{|c|c|c|c|c|c|c|c|c|c|}
\hline \multirow[t]{2}{*}{ Variables } & \multicolumn{2}{|c|}{$\underline{\text { Full sample }}$} & \multicolumn{2}{|c|}{ Adopters } & \multicolumn{2}{|c|}{$\underline{\text { Non-adopters }}$} & \multicolumn{2}{|c|}{ Difference in means } & \multirow[t]{2}{*}{ p-value } \\
\hline & Mean & SD & Mean & $\mathrm{SD}$ & Mean & SD & Mean & $\mathrm{SD}^{\mathrm{a}}$ & \\
\hline Age & 38.97 & 12.84 & 39.56 & 10.29 & 38.81 & 13.45 & -0.75 & 0.84 & 0.37 \\
\hline Gender & 0.83 & 0.37 & 0.86 & 0.34 & 0.82 & 0.38 & -0.04 & 0.02 & 0.14 \\
\hline Education (years) & 2.33 & 1.17 & 2.62 & 1.15 & 2.25 & 1.16 & -0.37 & 0.08 & 0.00 \\
\hline Family size & 6.66 & 2.69 & 7.45 & 3.06 & 6.45 & 2.54 & -1.01 & 0.17 & 0.00 \\
\hline Dependency ratio & 0.50 & 0.20 & 0.48 & 0.19 & 0.50 & 0.21 & 0.01 & 0.01 & 0.26 \\
\hline Land size in qarxi & 8.29 & 8.59 & 10.12 & 14.07 & 7.79 & 6.21 & -2.34 & 0.56 & 0.00 \\
\hline Livestock size (TLU) & 9.08 & 7.80 & 10.93 & 10.51 & 8.58 & 6.79 & -2.35 & 0.51 & 0.00 \\
\hline Distance from market & 1.69 & 0.99 & 1.87 & 1.10 & 1.64 & 0.95 & -0.23 & 0.06 & 0.00 \\
\hline Extension contact & 0.95 & 0.23 & 1.00 & 0.06 & 0.93 & 0.25 & -0.06 & 0.01 & 0.00 \\
\hline Investment in fertilizer & 1900.99 & 2424.94 & 3067.56 & 3187.98 & 1580.88 & 2058.42 & -1486.68 & 153.51 & 0.00 \\
\hline Investment in improved seed & 1542.15 & 3360.66 & 3104.9 & 5814.00 & 1113.32 & 2070.83 & -1991.58 & 213.22 & 0.00 \\
\hline Investment in pesticides & 226.00 & 1262.76 & 555.27 & 2586.66 & 135.65 & 403.81 & -419.61 & 81.83 & 0.00 \\
\hline Total investment in high-risk high-return inputs & 3669.14 & 4983.07 & 6727.72 & 8044.12 & 2829.86 & 3265.57 & -3897.86 & 308.66 & 0.00 \\
\hline Per capita food consumption expenditure & 88.05 & 178.25 & 109.76 & 166.13 & 82.09 & 181.05 & -27.67 & 11.64 & 0.02 \\
\hline Per capita non-food consumption expenditure & 1896.43 & 4000.30 & 2445.65 & 5537.43 & 1745.72 & 3449.41 & -699.93 & 261.02 & 0.01 \\
\hline Per capita total consumption expenditure & 6088.49 & 10556.83 & 7856.27 & 10840.61 & 5603.41 & 10430.41 & -2252.85 & 687.95 & 0.00 \\
\hline
\end{tabular}

$$
{ }^{\mathrm{a}} S D_{\text {difference in means }}=\sqrt{\frac{S D_{1}^{2}}{N_{1}}+\frac{S D_{2}^{2}}{N_{2}}}
$$


Our descriptive statistic results also show that IBI-adopters travel more distance to access markets and, also make more frequent contact with extension agents as compared with nonadopter households. Table 5.1 also shows that IBI-adopter and non-adopter households have significant differences in terms of the outcome variables. Compared to non-adopters, IBIadopters have made much more investment in total high-risk high-return agricultural inputs. On disaggregated terms, adopters have made much more investment in chemical fertilizer, improved seed varieties and pesticide/herbicides as compared with non-adopters. Similarly, there are statistically significant differences between IBI-adopter and non-adopter households in their per capita consumption. As shown in Table 5.1, on average, IBI-adopters have higher per capita weekly food consumption and higher per capita total annual consumption than IBI non-adopters. The difference in per capita non-food consumption between IBI-adopters and non-adopters is also statistically significant, showing that adopters have higher levels of consumption than non-adopters.

In general, Table 5.1 reveals that IBI-adopter and non-adopter households were observed to have statistically meaningful differences in some characteristics as well as outcome variables. These differences in characteristics between the two groups necessitate controlling for these variables in our subsequent impact estimate regressions. Similarly, the observed differences in outcome variables provide us with indications about the impacts. However, this measure of differences did not take into account the role of other covariates. As such these results are not adequate to causally infer the impact of IBI adoption on the welfare of adopter households. Hence, more rigorous estimates that take the role of other covariates into account were provided and discussed in the subsequent sections.

\subsubsection{Effects of participation in IBI adoption on household welfare}

Table 5.2 presents the impact of IBI adoption on household production behaviour. Estimated results were reported for value of high-risk high-return inputs, chemical fertilizer, improved seed variety and herbicide/pesticide, with and without controlling for potential covariates. Since we are primarily interested to measure the welfare impact of IBI adoption, we include only household observables that may systematically correlate with selection. Moreover, since time-invariant characteristics are removed by the within transformation, only time-varying variables were included in our estimation procedure. Demographic characteristics including age, gender and level of education of the household head as well as family size and dependency ratio are considered. 
Table 5.2: Impacts of IBI adoption on household production behaviour (difference-in-difference) estimates

\begin{tabular}{|c|c|c|c|c|c|c|c|c|}
\hline Variables & $\begin{array}{l}\text { (1) } \\
\text { Total value of } \\
\text { high-risk high- } \\
\text { return inputs }\end{array}$ & $\begin{array}{l}\text { (2) } \\
\text { Total value of } \\
\text { high-risk high- } \\
\text { return inputs }\end{array}$ & $\begin{array}{c}(3) \\
\text { Value of } \\
\text { fertilizers } \\
\text { used }\end{array}$ & $\begin{array}{c}(4) \\
\text { Value of } \\
\text { fertilizers } \\
\text { used }\end{array}$ & $\begin{array}{c}(5) \\
\text { Value of } \\
\text { improved } \\
\text { seed }\end{array}$ & $\begin{array}{c}(6) \\
\text { Value of } \\
\text { improved } \\
\text { seed }\end{array}$ & $\begin{array}{c}\text { (7) } \\
\text { Value of } \\
\text { pesticides } \\
\text { used }\end{array}$ & $\begin{array}{c}(8) \\
\text { Value of } \\
\text { pesticides } \\
\text { used }\end{array}$ \\
\hline Uptake of IBI & $\begin{array}{c}1,625.34^{* * * *} \\
(592.29)\end{array}$ & $\begin{array}{c}968.72 \\
(590.28)\end{array}$ & $\begin{array}{c}1,142.66^{* * *} \\
(338.53)\end{array}$ & $\begin{array}{c}753.73^{* *} \\
(305.66)\end{array}$ & $\begin{array}{c}190.90 \\
(147.57)\end{array}$ & $\begin{array}{c}-48.58 \\
(178.53)\end{array}$ & $\begin{array}{c}291.78 \\
(284.00)\end{array}$ & $\begin{array}{c}263.57 \\
(294.78)\end{array}$ \\
\hline Post & $\begin{array}{c}1,216.21^{* * *} \\
(207.30)\end{array}$ & $\begin{array}{c}1,194.02^{* * * *} \\
(215.74)\end{array}$ & $\begin{array}{c}119.10 \\
(134.73)\end{array}$ & $\begin{array}{c}98.48 \\
(138.88)\end{array}$ & $\begin{array}{c}1,046.43^{* * *} \\
(138.78)\end{array}$ & $\begin{array}{c}1,048.38^{* * *} \\
(142.99)\end{array}$ & $\begin{array}{c}50.68^{* *} \\
(22.13)\end{array}$ & $\begin{array}{c}47.16^{* *} \\
(23.31)\end{array}$ \\
\hline IBI*Post & $\begin{array}{c}4,545.06^{* * *} \\
(661.50)\end{array}$ & $\begin{array}{c}4,566.57 * * * \\
(667.04)\end{array}$ & $\begin{array}{l}688.03^{*} \\
(380.56)\end{array}$ & $\begin{array}{l}707.14 * \\
(382.76)\end{array}$ & $\begin{array}{c}3,601.35^{* * *} \\
(636.76)\end{array}$ & $\begin{array}{c}3,605.20 * * * \\
(640.93)\end{array}$ & $\begin{array}{c}255.68 \\
(296.18)\end{array}$ & $\begin{array}{c}254.23 \\
(300.49)\end{array}$ \\
\hline Region & & $\begin{array}{c}107.85 \\
(235.73)\end{array}$ & & $\begin{array}{c}173.46 \\
(174.18)\end{array}$ & & $\begin{array}{c}-73.38 \\
(150.45)\end{array}$ & & $\begin{array}{c}7.77 \\
(49.93)\end{array}$ \\
\hline Age & & $\begin{array}{c}9.64 \\
(12.40)\end{array}$ & & $\begin{array}{c}8.09 \\
(5.53)\end{array}$ & & $\begin{array}{c}-0.40 \\
(10.00)\end{array}$ & & $\begin{array}{c}1.94 \\
(1.70)\end{array}$ \\
\hline Sex & & $\begin{array}{c}-97.56 \\
(239.21)\end{array}$ & & $\begin{array}{l}-243.71 \\
(159.84)\end{array}$ & & $\begin{array}{c}218.05 \\
(149.55)\end{array}$ & & $\begin{array}{l}-71.91 * \\
(42.42)\end{array}$ \\
\hline Education & & $\begin{array}{c}520.52 * * * \\
(125.33)\end{array}$ & & $\begin{array}{c}343.78^{* * * *} \\
(76.81)\end{array}$ & & $\begin{array}{l}118.06^{*} \\
(60.97)\end{array}$ & & $\begin{array}{c}58.68 \\
(46.13)\end{array}$ \\
\hline Family size & & $\begin{array}{c}129.25^{*} \\
(74.59)\end{array}$ & & $\begin{array}{c}37.02 \\
(31.90)\end{array}$ & & $\begin{array}{c}84.96 \\
(67.44)\end{array}$ & & $\begin{array}{c}7.27 \\
(12.93)\end{array}$ \\
\hline Dependency ratio & & $\begin{array}{c}542.49 \\
(584.36)\end{array}$ & & $\begin{array}{c}85.59 \\
(283.49)\end{array}$ & & $\begin{array}{c}315.88 \\
(432.45)\end{array}$ & & $\begin{array}{c}141.02 \\
(139.76)\end{array}$ \\
\hline Land size & & $\begin{array}{c}42.93 * * \\
(17.48)\end{array}$ & & $\begin{array}{c}34.75^{* * *} \\
(8.20)\end{array}$ & & $\begin{array}{l}11.15 \\
(9.61)\end{array}$ & & $\begin{array}{l}-2.97 \\
(4.80)\end{array}$ \\
\hline TLU & & $\begin{array}{c}82.07 * * * \\
(17.81)\end{array}$ & & $\begin{array}{c}52.32 * * * \\
(11.23)\end{array}$ & & $\begin{array}{c}30.98 * * * \\
(10.90)\end{array}$ & & $\begin{array}{c}-1.23 \\
(3.95)\end{array}$ \\
\hline Distance from market & & $\begin{array}{l}-126.15 \\
(113.59)\end{array}$ & & $\begin{array}{l}-88.34 \\
(68.14)\end{array}$ & & $\begin{array}{l}-13.54 \\
(67.64)\end{array}$ & & $\begin{array}{l}-24.26 \\
(19.88)\end{array}$ \\
\hline Extension contact & & $\begin{array}{c}-256.98 \\
(504.10)\end{array}$ & & $\begin{array}{l}-255.36 \\
(361.00)\end{array}$ & & $\begin{array}{c}-26.71 \\
(222.10)\end{array}$ & & $\begin{array}{c}25.09 \\
(46.50)\end{array}$ \\
\hline District 1 & & $\begin{array}{c}704.98 \\
(435.70)\end{array}$ & & $\begin{array}{c}1,081.79^{* * * *} \\
(264.00)\end{array}$ & & $\begin{array}{c}-647.29 * * \\
(320.27)\end{array}$ & & $\begin{array}{c}270.48 \\
(275.18)\end{array}$ \\
\hline District 2 & & $\begin{array}{l}-150.91 \\
(390.44)\end{array}$ & & $\begin{array}{c}279.73^{*} \\
(142.24)\end{array}$ & & $\begin{array}{c}-388.53 \\
(353.73)\end{array}$ & & $\begin{array}{l}-42.11 \\
(55.42)\end{array}$ \\
\hline Constant & $\begin{array}{c}2,221.75^{* * *} \\
(135.28)\end{array}$ & $\begin{array}{c}-1,029.53 \\
(1,000.37)\end{array}$ & $\begin{array}{c}1,521.33 * * * \\
(108.38)\end{array}$ & $\begin{array}{l}-484.10 \\
(600.32)\end{array}$ & $\begin{array}{c}590.11^{* * * *} \\
(65.66)\end{array}$ & $\begin{array}{l}-414.03 \\
(668.21)\end{array}$ & $\begin{array}{c}110.31 * * * \\
(16.29)\end{array}$ & $\begin{array}{l}-131.41 \\
(241.53)\end{array}$ \\
\hline Observations & 1,384 & 1,384 & 1,384 & 1,384 & 1,384 & 1,384 & 1,384 & 1,384 \\
\hline R-squared & 0.19 & 0.23 & 0.07 & 0.16 & 0.18 & 0.20 & 0.02 & 0.03 \\
\hline
\end{tabular}

Note: $* * * \mathrm{p}<0.01, * * \mathrm{p}<0.05, * \mathrm{p}<0.1$ Robust standards errors, clustered at Iddir level are in brackets. Estimations follow OLS regressions based on eq. (5.1). 
Land size and size of the livestock owned by the household were also controlled for, since these productive assets may also affect the level of the outcome indicators. In anticipation that OIC might have targeted accessible villages in terms of infrastructure, we included proxies, distance from the market and frequency of extension contact, as two sets of controls. Moreover, we controlled for spatial variations by including district dummies in the regressions. The full estimate results based on the difference-in-difference show that adoption of IBI has a significant positive effect on household investment in high-risk highreturn agricultural inputs for adopters as compared with non-adopters. After controlling for potential selection on unobservables, household investment in high-risk high-return agricultural inputs has increased by ETB 4,567 for IBI-adopters, compared to non-adopters (see Column 2 in Table 5.2). Disaggregating these results, Table 5.2 indicates adoption of IBI have statistically significant impact on increasing investment in improved seed variety, but not on investment in chemical fertilizer and pesticide/herbicide. With the full estimate including all covariates, uptake of IBI increases investment in improved seed varieties by ETB 3,605. The results are statistically significant at 1 percent level. Note that the doubledifferencing estimation procedure reduces the risk of selection bias.

Two causal mechanisms can be proposed. First, household can pursue intensification with increased investment on insured farms with the expectation that increase in productivity provides for a higher return. Second, households can increase their investment in inputs as they expect that payout from the insurance firm can well commensurate their investment. Table 5.2 also shows that certain control variables, namely education of the household, land size and number of livestock owned by the household have statistically significant positive effect on investment in high-risk high-return inputs.

Table 5.3 presents the results of double difference estimates of the impact of IBI adoption on household food, non-food and total consumption, with and without controlling for potential covariates. The full estimate with covariates indicates that adoption of IBI increases household per capita weekly food consumption expenditure by ETB 48 (see Column 2 in Table 5.3). Similarly, per capita annual consumption is increased by ETB 3,006 for IBIadopters than non-adopters (see Table 5.3 under Column 6 for the details).

Table 5.3 also shows that consistent with theory, our estimation also shows household consumption decreases with increase in dependency ratio and family size, and consumption increases with household assets like increase in number of livestock owned. 
Table 5.3: Impacts of IBI adoption on household consumption (difference-in-difference estimates)

\begin{tabular}{|c|c|c|c|c|c|c|}
\hline Variables & $\begin{array}{l}\text { (1) } \\
\text { Per capita food } \\
\text { consumption } \\
\text { expenditure }\end{array}$ & $\begin{array}{c}(2) \\
\text { Per capita food } \\
\text { consumption } \\
\text { expenditure }\end{array}$ & $\begin{array}{l}(3) \\
\text { Per capita non-food } \\
\text { consumption } \\
\text { expenditure }\end{array}$ & $\begin{array}{l}(4) \\
\text { Per capita non-food } \\
\text { consumption } \\
\text { expenditure }\end{array}$ & $\begin{array}{c}\text { (5) } \\
\text { Per capita total } \\
\text { consumption } \\
\text { expenditure }\end{array}$ & $\begin{array}{c}6) \\
\text { Per capita total } \\
\text { consumption expenditure }\end{array}$ \\
\hline Uptake of IBI & $\begin{array}{c}3.69 \\
(2.80)\end{array}$ & $\begin{array}{c}1.36 \\
(5.31)\end{array}$ & $\begin{array}{l}602.83^{*} \\
(314.22)\end{array}$ & $\begin{array}{l}605.46^{*} \\
(358.62)\end{array}$ & $\begin{array}{l}757.92^{*} \\
(447.12)\end{array}$ & $\begin{array}{c}626.43 \\
(577.18)\end{array}$ \\
\hline Post & $\begin{array}{c}87.30^{* * * *} \\
(12.25)\end{array}$ & $\begin{array}{l}88.00 * * * \\
(12.76)\end{array}$ & $\begin{array}{c}794.86^{* * * *} \\
(181.65)\end{array}$ & $\begin{array}{c}822.29 * * * \\
(184.92)\end{array}$ & $\begin{array}{c}4,254.87^{* * * *} \\
(691.05)\end{array}$ & $\begin{array}{c}4,317.88^{* * * *} \\
(713.09)\end{array}$ \\
\hline IBI*Post & $\begin{array}{l}47.95 * * \\
(22.09)\end{array}$ & $\begin{array}{l}48.28^{* *} \\
(22.42)\end{array}$ & $\begin{array}{c}194.19 \\
(538.15)\end{array}$ & $\begin{array}{c}197.97 \\
(538.57)\end{array}$ & $\begin{array}{c}2,989.86^{* *} \\
(1,241.46)\end{array}$ & $\begin{array}{c}3,006.83 * * \\
(1,255.42)\end{array}$ \\
\hline Region & & $\begin{array}{c}23.82 \\
(14.86)\end{array}$ & & $\begin{array}{l}457.47^{*} \\
(258.71)\end{array}$ & & $\begin{array}{c}1,510.19^{*} \\
(841.72)\end{array}$ \\
\hline Age & & $\begin{array}{l}-0.55^{*} \\
(0.32)\end{array}$ & & $\begin{array}{l}-18.49 \\
(11.17)\end{array}$ & & $\begin{array}{c}-40.86^{* *} \\
(20.16)\end{array}$ \\
\hline Sex & & $\begin{array}{c}6.77 \\
(17.05)\end{array}$ & & $\begin{array}{c}84.42 \\
(303.58)\end{array}$ & & $\begin{array}{c}598.32 \\
(961.95)\end{array}$ \\
\hline Education & & $\begin{array}{c}2.36 \\
(3.83)\end{array}$ & & $\begin{array}{l}328.92 * * \\
(135.07)\end{array}$ & & $\begin{array}{c}363.62 \\
(239.86)\end{array}$ \\
\hline Family size & & $\begin{array}{c}-11.13 * * * \\
(1.36)\end{array}$ & & $\begin{array}{c}-291.01 * * * \\
(37.41)\end{array}$ & & $\begin{array}{c}-814.24 * * * \\
(91.69)\end{array}$ \\
\hline Dependency ratio & & $\begin{array}{c}-93.40 * * \\
(37.47)\end{array}$ & & $\begin{array}{c}-2,891.37 * * * \\
(795.03)\end{array}$ & & $\begin{array}{c}-7,246.75 * * * \\
(2,250.66)\end{array}$ \\
\hline Land size & & $\begin{array}{l}-0.13 \\
(0.35)\end{array}$ & & $\begin{array}{l}-11.73 \\
(8.15)\end{array}$ & & $\begin{array}{c}-8.42 \\
(21.94)\end{array}$ \\
\hline TLU & & $\begin{array}{l}1.01 * * \\
(0.49)\end{array}$ & & $\begin{array}{c}29.16^{* *} \\
(14.34)\end{array}$ & & $\begin{array}{l}92.02 * * \\
(36.81)\end{array}$ \\
\hline Distance from market & & $\begin{array}{l}1.47 \\
(3.77)\end{array}$ & & $\begin{array}{c}30.15 \\
(138.69)\end{array}$ & & $\begin{array}{c}88.28 \\
(252.42)\end{array}$ \\
\hline Extension contact & & $\begin{array}{l}-15.10 \\
(23.91)\end{array}$ & & $\begin{array}{l}-394.72 \\
(480.11)\end{array}$ & & $\begin{array}{c}-700.08 \\
(1,251.45)\end{array}$ \\
\hline District1 & & $\begin{array}{l}21.43 \\
(14.06)\end{array}$ & & $\begin{array}{c}1,393.29 * * * \\
(429.42)\end{array}$ & & $\begin{array}{c}1,878.41^{* *} \\
(862.40)\end{array}$ \\
\hline District2 & & $\begin{array}{c}-6.02 \\
(12.74)\end{array}$ & & $\begin{array}{c}604.42 * * \\
(256.08)\end{array}$ & & $\begin{array}{c}311.33 \\
(744.78)\end{array}$ \\
\hline Constant & $\begin{array}{c}38.44 * * * \\
(1.42)\end{array}$ & $\begin{array}{l}155.45 * * * \\
(29.50)\end{array}$ & $\begin{array}{c}1,348.29 * * * \\
(76.22)\end{array}$ & $\begin{array}{l}3,744.05 * * * \\
(978.22)\end{array}$ & $\begin{array}{c}3,475.98 * * * \\
(215.95)\end{array}$ & $\begin{array}{c}10,815.80 * * * \\
(2,063.67)\end{array}$ \\
\hline Observations & 1,384 & 1,384 & 1,384 & 1,384 & 1,384 & 1,384 \\
\hline R-squared & 0.08 & 0.16 & 0.02 & 0.15 & 0.06 & 0.19 \\
\hline
\end{tabular}

Note: ${ }^{* * *} \mathrm{p}<0.01, * * \mathrm{p}<0.05,{ }^{*} \mathrm{p}<0.1$ Robust standards errors, clustered at Iddir level are in brackets. Estimations follow OLS regressions based on eq. (5.1). 


\subsubsection{Effects of intensity of IBI uptake on household welfare}

An important follow-up question is that we address in this section is the extent to which the impact of IBI adoption varies with the intensity and frequency of repeated adoptions. Instead of mere participation in IBI adoption, in this case, indicators were assigned, for the number of years that each household has been involved in IBI adoption phase. Table 5.4 provides the estimated results of this model for the first category of outcome indicators related to investment in high-risk high-return inputs. Again note that the double-differencing estimation procedure reduces the risk of selection bias. Results in Table 5.4 show once again that IBI adoption has a significant impact on household investment in high-risk high-return agricultural inputs. Interestingly, Table 5.4 shows that the magnitude of the impact of IBI adoption increases with increase in frequency (years) of adoption.

Controlling for all covariates, adoption of IBI for two years have increased total investment in high-risk high-return agricultural inputs by ETB 2248 (See Column 2 in Table 5.4). Further, three- and four- years of adoption of IBI have increased total investment in high-risk high-return inputs by ETB 5,562 and ETB 4,746 (both with $p=0.00$ ). Table 5.4 also show that for individual input components, two-, three- and four-years of adoption of IBI increase investment in chemical fertilizers by ETB 852, ETB 1643 and ETB 1597, respectively. These results are statistically significant at 1\% level controlling for all covariates (See Column 4 in Table 5.4). Similarly, controlling for all covariates, two-, three- and four-years of adoption of IBI were observed to increase the respective investment in improved seed variety by ETB 1,296, ETB 2,757 and ETB 2,653, and the investment in pesticide/herbicide by ETB 100, ETB 1162 and ETB 495, respectively.

Next, the impact of repeated IBI adoption on per capita consumption is presented in Table 5.5. Overall results reveal that the impact of IBI adoption on household per capita food and total consumption increases with the number of years of adoption. Three- and four-years of adoption were indicated to increase household per capita weekly food consumption expenditure by ETB 73 and 52, respectively. Three-years of adoption was also indicated to increase per capita total consumption expenditure by ETB 4080. As their income increases, households may spend more on basic consumption items (e.g., food) at first, followed by investment in high-risk high-return inputs (e.g., purchase of chemical fertilizer, improved seed varieties and use of pesticide/herbicides). And, then, households may invest in other consumption items (e.g., health, education or clothing). 
Table 5.4: The impact of intensity of IBI adoption on household production behaviour (difference-in-difference estimates)

\begin{tabular}{|c|c|c|c|c|c|c|c|c|}
\hline Variables & $\begin{array}{l}\text { (1) } \\
\text { Total value of high- } \\
\text { risk high-return } \\
\text { inputs }\end{array}$ & $\begin{array}{l}\text { (2) } \\
\text { Total value of } \\
\text { high-risk high- } \\
\text { return inputs }\end{array}$ & $\begin{array}{c}(3) \\
\text { Value of } \\
\text { fertilizers } \\
\text { used }\end{array}$ & $\begin{array}{c}(4) \\
\text { Value of } \\
\text { fertilizers } \\
\text { used }\end{array}$ & $\begin{array}{c}(5) \\
\text { Value of } \\
\text { improved } \\
\text { seed }\end{array}$ & $\begin{array}{c}(6) \\
\text { Value of } \\
\text { improved } \\
\text { seed }\end{array}$ & $\begin{array}{c}(7) \\
\text { Value of } \\
\text { pesticides } \\
\text { used }\end{array}$ & $\begin{array}{c}\text { (8) } \\
\text { Value of } \\
\text { pesticides used }\end{array}$ \\
\hline Two-years adoption & $\begin{array}{c}2,684.45^{* * * *} \\
(570.20)\end{array}$ & $\begin{array}{c}2,247.66^{* * * *} \\
(576.57)\end{array}$ & $\begin{array}{c}1,009.46^{* * *} \\
(215.51)\end{array}$ & $\begin{array}{c}851.91 * * * \\
(198.14)\end{array}$ & $\begin{array}{c}1,574.55 * * * \\
(501.92)\end{array}$ & $\begin{array}{c}1,296.25^{* *} \\
(501.66)\end{array}$ & $\begin{array}{c}100.43 * * * \\
(30.34)\end{array}$ & $\begin{array}{l}99.50 * * * \\
(32.31)\end{array}$ \\
\hline Three-years adoption & $\begin{array}{c}6,014.70^{* * *} \\
(1,135.43)\end{array}$ & $\begin{array}{c}5,561.76^{* * * *} \\
(1,068.79)\end{array}$ & $\begin{array}{c}1,985.18 * * * \\
(417.34)\end{array}$ & $\begin{array}{c}1,642.89 * * * \\
(340.28)\end{array}$ & $\begin{array}{c}2,842.10 * * * \\
(486.31)\end{array}$ & $\begin{array}{c}2,756.59 * * * \\
(499.20)\end{array}$ & $\begin{array}{c}1,187.43^{*} \\
(601.01)\end{array}$ & $\begin{array}{l}1,162.28 * * \\
(567.05)\end{array}$ \\
\hline Four-years adoption & $\begin{array}{c}5,353.53 * * * \\
(1,108.31)\end{array}$ & $\begin{array}{c}4,745.78^{* * * *} \\
(1,077.15)\end{array}$ & $\begin{array}{c}2,031.89^{* * * *} \\
(609.82)\end{array}$ & $\begin{array}{c}1,596.95^{* *} \\
(652.40)\end{array}$ & $\begin{array}{c}2,900.51^{* *} \\
(1,124.40)\end{array}$ & $\begin{array}{c}2,653.42 * * * \\
(998.81)\end{array}$ & $\begin{array}{c}421.14 * * * \\
(114.14)\end{array}$ & $\begin{array}{c}495.42 * * * \\
(139.20)\end{array}$ \\
\hline Region & $\begin{array}{r}480.97 * * \\
(212.89)\end{array}$ & $\begin{array}{c}74.68 \\
(225.95)\end{array}$ & $\begin{array}{c}422.67 * * * \\
(157.90)\end{array}$ & $\begin{array}{l}168.05 \\
(169.99)\end{array}$ & $\begin{array}{c}-27.18 \\
(128.90)\end{array}$ & $\begin{array}{l}-103.93 \\
(143.94)\end{array}$ & $\begin{array}{c}85.48 * * * \\
(20.07)\end{array}$ & $\begin{array}{c}10.56 \\
(47.42)\end{array}$ \\
\hline Age & & $\begin{array}{l}20.38^{*} \\
(11.30)\end{array}$ & & $\begin{array}{l}9.46^{*} \\
(5.53)\end{array}$ & & $\begin{array}{c}8.50 \\
(9.29)\end{array}$ & & $\begin{array}{c}2.42 \\
(1.48)\end{array}$ \\
\hline Sex & & $\begin{array}{c}-76.97 \\
(237.55)\end{array}$ & & $\begin{array}{l}-227.34 \\
(163.03)\end{array}$ & & $\begin{array}{c}202.74 \\
(145.53)\end{array}$ & & $\begin{array}{l}-52.37 \\
(35.07)\end{array}$ \\
\hline Education (years) & & $\begin{array}{l}589.40 * * * \\
(112.88)\end{array}$ & & $\begin{array}{l}354.52 * * * \\
(74.00)\end{array}$ & & $\begin{array}{c}170.92 * * * \\
(58.53)\end{array}$ & & $\begin{array}{c}63.96 \\
(43.18)\end{array}$ \\
\hline Family size & & $\begin{array}{c}94.75 \\
(70.44)\end{array}$ & & $\begin{array}{c}30.59 \\
(31.75)\end{array}$ & & $\begin{array}{c}63.23 \\
(64.31)\end{array}$ & & $\begin{array}{c}0.93 \\
(11.62)\end{array}$ \\
\hline Dependency ratio & & $\begin{array}{c}681.82 \\
(542.55)\end{array}$ & & $\begin{array}{c}101.69 \\
(289.28)\end{array}$ & & $\begin{array}{c}444.14 \\
(399.16)\end{array}$ & & $\begin{array}{c}135.98 \\
(118.80)\end{array}$ \\
\hline Land size & & $\begin{array}{l}41.94 * * \\
(17.75)\end{array}$ & & $\begin{array}{c}34.90 * * * \\
(8.20)\end{array}$ & & $\begin{array}{c}9.44 \\
(9.63)\end{array}$ & & $\begin{array}{l}-2.40 \\
(4.54)\end{array}$ \\
\hline TLU & & $\begin{array}{c}76.23 * * * \\
(19.00)\end{array}$ & & $\begin{array}{c}50.54 * * * \\
(11.84)\end{array}$ & & $\begin{array}{l}27.64 * * \\
(11.38)\end{array}$ & & $\begin{array}{l}-1.95 \\
(3.87)\end{array}$ \\
\hline Distance from the market & & $\begin{array}{l}-151.38 \\
(108.81)\end{array}$ & & $\begin{array}{l}-94.96 \\
(68.12)\end{array}$ & & $\begin{array}{l}-25.47 \\
(64.27)\end{array}$ & & $\begin{array}{l}-30.95 \\
(20.28)\end{array}$ \\
\hline Extension contact & & $\begin{array}{l}-414.28 \\
(490.15)\end{array}$ & & $\begin{array}{l}-273.43 \\
(355.06)\end{array}$ & & $\begin{array}{l}-159.64 \\
(217.33)\end{array}$ & & $\begin{array}{c}18.80 \\
(38.41)\end{array}$ \\
\hline District 1 & & $\begin{array}{c}302.93 \\
(406.89)\end{array}$ & & $\begin{array}{c}983.28 * * * \\
(272.48)\end{array}$ & & $\begin{array}{c}-822.36^{* * * *} \\
(274.01)\end{array}$ & & $\begin{array}{c}142.01 \\
(207.69)\end{array}$ \\
\hline District 2 & & $\begin{array}{l}-414.92 \\
(351.45)\end{array}$ & & $\begin{array}{c}214.88 \\
(138.97)\end{array}$ & & $\begin{array}{l}-524.61 * \\
(309.67)\end{array}$ & & $\begin{array}{c}-105.19 * * \\
(49.07)\end{array}$ \\
\hline Constant & $\begin{array}{c}2,518.07 * * * \\
(167.99)\end{array}$ & $\begin{array}{l}-344.30 \\
(943.90)\end{array}$ & $\begin{array}{c}1,306.89 * * * \\
(107.31)\end{array}$ & $\begin{array}{l}-383.69 \\
(582.73)\end{array}$ & $\begin{array}{c}1,130.95 * * * \\
(102.57)\end{array}$ & $\begin{array}{c}65.87 \\
(639.59)\end{array}$ & $\begin{array}{c}80.24 * * * \\
(14.50)\end{array}$ & $\begin{array}{c}-26.49 \\
(192.65)\end{array}$ \\
\hline $\begin{array}{l}\text { Observations } \\
\text { R-squared }\end{array}$ & $\begin{array}{c}1,384 \\
0.12\end{array}$ & $\begin{array}{c}1,384 \\
0.17\end{array}$ & $\begin{array}{c}1,384 \\
0.08\end{array}$ & $\begin{array}{c}1,384 \\
0.16\end{array}$ & $\begin{array}{c}1,384 \\
0.07\end{array}$ & $\begin{array}{c}1,384 \\
0.09\end{array}$ & $\begin{array}{c}1,384 \\
0.05\end{array}$ & $\begin{array}{c}1,384 \\
0.05\end{array}$ \\
\hline
\end{tabular}


Table 5.5: The impact of intensity of IBI adoption on household per capita consumption (difference-in-difference estimates)

\begin{tabular}{|c|c|c|c|c|c|c|}
\hline Variables & $\begin{array}{l}\text { (1) } \\
\text { Per capita weekly } \\
\text { food consumption } \\
\text { expenditure }\end{array}$ & $\begin{array}{l}\text { (2) } \\
\text { Per capita weekly } \\
\text { food consumption } \\
\text { expenditure }\end{array}$ & $\begin{array}{l}(3) \\
\text { Per capita non-food } \\
\text { consumption } \\
\text { expenditure }\end{array}$ & $\begin{array}{l}\text { (4) } \\
\text { Per capita non-food } \\
\text { consumption } \\
\text { expenditure }\end{array}$ & $\begin{array}{c}(5) \\
\text { Per capita total } \\
\text { consumption } \\
\text { expenditure }\end{array}$ & $\begin{array}{c}\text { (6) } \\
\text { Per capita total } \\
\text { consumption expenditure }\end{array}$ \\
\hline Two-years adoption & $\begin{array}{c}-7.52 \\
(13.75)\end{array}$ & $\begin{array}{c}6.35 \\
(11.70)\end{array}$ & $\begin{array}{c}344.08 \\
(538.17)\end{array}$ & $\begin{array}{c}709.31 \\
(546.54)\end{array}$ & $\begin{array}{c}159.68 \\
(974.34)\end{array}$ & $\begin{array}{l}1,078.49 \\
(883.74)\end{array}$ \\
\hline Three-years adoption & $\begin{array}{c}52.02 \\
(31.65)\end{array}$ & $\begin{array}{l}72.75^{* *} \\
(30.04)\end{array}$ & $\begin{array}{l}-125.82 \\
(343.41)\end{array}$ & $\begin{array}{l}309.69 \\
(330.25)\end{array}$ & $\begin{array}{c}2,662.60 \\
(1,736.97)\end{array}$ & $\begin{array}{l}4,079.79 * * \\
(1,632.62)\end{array}$ \\
\hline Four-years adoption & $\begin{array}{c}39.34 \\
(32.50)\end{array}$ & $\begin{array}{l}52.17 * * \\
(24.38)\end{array}$ & $\begin{array}{c}1,665.21 \\
(1,892.54)\end{array}$ & $\begin{array}{c}1,837.99 \\
(1,682.33)\end{array}$ & $\begin{array}{c}4,707.73 \\
(3,379.24)\end{array}$ & $\begin{array}{l}5,289.44^{*} \\
(2,744.59)\end{array}$ \\
\hline Region & $\begin{array}{l}47.20 * * * \\
(9.93)\end{array}$ & $\begin{array}{c}22.39 \\
(14.88)\end{array}$ & $\begin{array}{c}977.05 * * * \\
(165.14)\end{array}$ & $\begin{array}{c}429.27 \\
(261.75)\end{array}$ & $\begin{array}{l}3,040.52 * * * \\
(576.22)\end{array}$ & $\begin{array}{l}1,414.87^{*} \\
(844.40)\end{array}$ \\
\hline Age & & $\begin{array}{l}-0.08 \\
(0.30)\end{array}$ & & $\begin{array}{l}-13.85 \\
(10.78)\end{array}$ & & $\begin{array}{l}-16.14 \\
(18.63)\end{array}$ \\
\hline Sex & & $\begin{array}{c}5.21 \\
(16.74)\end{array}$ & & $\begin{array}{c}67.58 \\
(300.02)\end{array}$ & & $\begin{array}{c}546.16 \\
(941.90)\end{array}$ \\
\hline Education (years) & & $\begin{array}{c}5.05 \\
(3.77)\end{array}$ & & $\begin{array}{c}356.11^{* *} \\
(137.50)\end{array}$ & & $\begin{array}{l}509.76^{* *} \\
(238.23)\end{array}$ \\
\hline Family size & & $\begin{array}{c}-12.28 * * * \\
(1.36)\end{array}$ & & $\begin{array}{c}-297.35 * * * \\
(37.90)\end{array}$ & & $\begin{array}{c}-872.66^{* * *} \\
(88.80)\end{array}$ \\
\hline Dependency ratio & & $\begin{array}{c}-85.97 * * \\
(36.10)\end{array}$ & & $\begin{array}{c}-2,793.35 * * * \\
(770.86)\end{array}$ & & $\begin{array}{c}-6,832.89 * * * \\
(2,169.34)\end{array}$ \\
\hline Land size & & $\begin{array}{l}-0.19 \\
(0.30)\end{array}$ & & $\begin{array}{l}-12.84 \\
(8.50)\end{array}$ & & $\begin{array}{l}-11.90 \\
(19.64)\end{array}$ \\
\hline TLU & & $\begin{array}{l}0.90^{*} \\
(0.51)\end{array}$ & & $\begin{array}{c}25.87 \\
(16.12)\end{array}$ & & $\begin{array}{l}81.20^{* *} \\
(39.66)\end{array}$ \\
\hline Distance from the market & & $\begin{array}{l}0.98 \\
(3.81)\end{array}$ & & $\begin{array}{c}27.98 \\
(145.18)\end{array}$ & & $\begin{array}{c}58.43 \\
(260.76)\end{array}$ \\
\hline Extension contact & & $\begin{array}{l}-24.20 \\
(23.85)\end{array}$ & & $\begin{array}{l}-487.27 \\
(481.02)\end{array}$ & & $\begin{array}{l}-1,168.34 \\
(1,250.64)\end{array}$ \\
\hline District1 & & $\begin{array}{c}13.36 \\
(13.65)\end{array}$ & & $\begin{array}{l}1,430.96^{* * * *} \\
(468.35)\end{array}$ & & $\begin{array}{l}1,495.78^{*} \\
(857.42)\end{array}$ \\
\hline District2 & & $\begin{array}{l}-11.99 \\
(12.57)\end{array}$ & & $\begin{array}{c}570.21 * * \\
(278.18)\end{array}$ & & $\begin{array}{c}-41.07 \\
(748.17)\end{array}$ \\
\hline Constant & $\begin{array}{c}51.49 * * * \\
(3.59)\end{array}$ & $\begin{array}{c}197.31 * * * \\
(30.60)\end{array}$ & $\begin{array}{c}1,112.35^{* * * *} \\
(77.35)\end{array}$ & $\begin{array}{c}4,080.90^{* * *} * \\
(1,012.38)\end{array}$ & $\begin{array}{c}3,632.39 * * * \\
(225.87)\end{array}$ & $\begin{array}{c}12,879.73 * * * \\
(2,091.72)\end{array}$ \\
\hline Observations & 1,384 & 1,384 & 1,384 & 1,384 & 1,384 & 1,384 \\
\hline R-squared & 0.02 & 0.09 & 0.02 & 0.14 & 0.03 & 0.13 \\
\hline
\end{tabular}

Note: $* * * \mathrm{p}<0.01, * * \mathrm{p}<0.05, * \mathrm{p}<0.1$ Robust standards errors, clustered at Iddir level are in brackets. Estimations follow OLS regressions based on eq. (5.2). 
Hence, it is likely that the cumulative effect of repeated adoption of IBI on household welfare is in line with the usual spending patterns of households as their income increases.

\subsection{Conclusion}

This study evaluates the impact of IBI adoption on two categories of welfare indicators: household consumption and investment in high-risk high-return inputs. The study used a tworound panel data collected from smallholders in the Rift Valley zone of south-eastern Ethiopia. IBI adoption in the area has lasted four years before this study, so that lasting welfare effects can be established. Self-selection and program placement biases often complicate causal attributions of welfare improvements to rural interventions. The study employs difference-in-difference techniques to reduce selections based on time-invariant unobservables. The results indicate that adoption of IBI has significantly increased both investment in high-risk high-return inputs and per capita household consumption expenditure, which are important indicators of welfare in the study area. A flexible specification of the double difference estimator that takes the frequency of adoption into account has also shown that investment in high-risk high-return inputs as well as per capita household consumption were increased with the frequency of IBI adoption. Repeated cycles of adoption, however, do matter to achieve significant welfare impacts from IBI adoption. Both methods strongly suggest that adoption of IBI in this specific Rift Valley zone of east Africa has been useful in terms of the measured household welfare outcomes. These findings have a number of implications. First, they show that the effect of IBI adoption on household welfare can be multidimensional and may not be fully captured by just a single household welfare outcome indicator. Second, the results also indicate that it takes time before the effect of IBI adoption on consumption or investment in high-risk high-return inputs is fully materialized. Therefore, impact estimates that rely on a single household welfare indicator and focus only on one cycle or year of IBI adoption may underestimate the potential welfare gains that can be achieved overtime. Future research should focus on larger longitudinal database with increase in both the number of years and units of observations. Finally, an important implication of these results for microinsurance firms like OIC is that IBI-adopter households should be encouraged not only to participate in adoption once, but also to sustain their uptake by renewing the IBI policy, and by remaining for longer periods in adoption phase in order to realize the full potentials in welfare gains. 
Appendix 6: Variable type and definition

Variables

Age

Gender

Education (years)

Family size

Dependency ratio

Land size in qarxi

Livestock size (TLU)

Distance from market

Extension contact

Investment in fertilizer

Investment in improved seed

Investment in

pesticides/herbicides

Total investment in high-risk highreturn inputs

Per capita food consumption expenditure

Per capita non-food consumption expenditure

Per capita total consumption expenditure

\section{Variable type and definition}

Continuous, age of the household head in years

Dummy, gender of the household head, $1=$ male headed $0=$ female headed

Continuous, household head's level of education in years of schooling

Continuous, number of household members in the family

Continuous, ratio of dependents ${ }^{1}$ in a family to family size

Continuous, household's land holding, measured in a local unit called qarxi, where 1 qarxi $=0.25$ hectares

Continuous, number of livestock owned by a household measured in standard tropical livestock units (TLU) ${ }^{2}$

Continuous, distance from household's residence to market measured in walking hours

Dummy, equal to 1 for households that frequently make contact with extension agents; 0 for others

Continuous, value of household's investment in fertilizer in ETB

Continuous, value of household's investment in improved seed varieties in ETB

Continuous, value of household's investment in pesticides/herbicides in ETB

Continuous, value of household's total investment in high-risk highreturn agricultural inputs in ETB

Continuous, value of household's annual per capita food consumption expenditure in ETB

Continuous, value of household's annual per capita non-food consumption expenditure in ETB

Continuous, value of household's total annual per capita food consumption expenditure in ETB

\footnotetext{
${ }^{1}$ Family dependents were counted as household members with age less than 15 years (preschool children) and those with age greater than 65

${ }^{2} 1 \mathrm{TLU}=1 *$ cow or ox $=0.75 *$ heifer $=0.34 *$ calf $=1.1 *$ horse $=0.7 *$ donkey $=0.13 *$ adult sheep or goat $=$ 0.013*chicken (Storck and Doppler 1991).
} 


\section{Chapter 6}

\section{Summary, conclusion and the way forward}

\subsection{Introduction}

Climate change induced risks cause magnificent welfare losses among smallholders in developing countries. The need for innovative risk management mechanisms is high on the agenda of development economics research and policy making. Insurance is the obvious policy option to manage risks. However, in the past periods, indemnity-based insurances that constitute the formal agricultural risk hedging markets were unsuccessful mainly due to problems associated with information asymmetry including moral hazard and adverse selection, and transaction costs. In recent years, innovative insurance instruments known as index-based insurances were developed to respond to the massive exposure of smallholders to uninsured covariate risks as well as their reinforcing impacts on persistent poverty.

Index-based insurances are innovative hedging instruments that mitigate drought shocks and seasonality-based weather risks induced by climate change. Payout is triggered when the index of a selective weather variable falls below a given threshold, signaling risk. Such index is often constructed from rainfall or vegetative cover on the surface of the earth, measured by satellite remote sensing. A reliable index to be used in index-based insurances should be closely correlated with the insured asset, objectively quantifiable and publicly verifiable in order not to be manipulated by both the insurer and the insured. Thus, in many ways, innovations in index-based insurances are helpful to overcome challenges that often plague the development of rural financial markets like the functioning of indemnity insurances. First, IBI delinks loss assessment from individual behaviour to overcome moral hazard problems. Second, the design of index-based insurances is based on publicly verifiable data like NDVI

data extracted from satellite measures. Thus, it partially tackles the problem of adverse selection. Third, the use of a single index to estimate losses of a group of farms minimizes transaction costs. In this way, IBIs uniquely overcome classic incentive problems like information asymmetry and transaction costs associated with claim verification and contract enforcement in rural financial markets. However, despite these merits, the uptake of indexbased insurances is very low in developing countries, typically ranging below $10 \%$. 
One of the main objectives of this dissertation is to examine the various possibilities to increase the uptake of IBI among smallholders to shape it to be a sustainable risk management tool. This thesis starts with examining whether the uptake of IBI of smallholders can be explained by their risk and ambiguity aversion behaviour. In addition, we carried out a randomized control trial (RCT) experiment to measure the extent to which relaxing two of the most theoretically impeding socio-economic constraints for insurance uptake, namely trust and liquidity constraints, can enhance the uptake of IBIs. The second main objective of this dissertation was to measure the welfare impacts of adopting IBIs for smallholders. We measured the welfare impacts of IBI adoption from various perspectives. First, we assessed the effect of IBI adoption on enhancing households' access to financial markets. Specifically, we measured the extent to which adoption of IBIs reduce credit rationing among smallholders in Ethiopia. Second, we systematically measured the ex-ante impact of IBI adoption on investment in high-risk high-return agricultural inputs. Specifically, we measured the ex-ante gains from adoption of IBI in terms of increased investment in chemical fertilizer, improved seed variety and use of pesticides and/or herbicides. Thirdly, we assessed the ex-post impact of IBI adoption on household food and non-food consumptions. Finally, this chapter summarizes the main findings in different pieces of the studies, draws conclusions, and discusses the implications in a wider context.

\subsection{Behavioural impediments in technology adoption}

In chapter 2 we examined how individual household-specific behavioural impediments influence IBI adoption decisions of smallholders in developing countries. Previous studies from the perspectives of behavioural economics noted that the main problem in adoption of IBI is basis risk-an imperfect correlation between the index and actual losses-that results in invalid trigger of the payout. Our study identifies that basis risk induces ambiguity in IBI uptake decisions of smallholders. Smallholders can remain ambiguous about the payout from IBI even after they incurred significant losses. Because of a higher potential degree of ambiguity associated with the failure of the IBI contract to accurately reflect loss realizations of smallholders, we hypothesized that ambiguity aversion might play a great role in hampering adoption of IBI in the study area. We tested this hypothesis using a discrete choice adoption model including experimental measures of risk and ambiguity aversion. We learned 
that ambiguity aversion has a large impact in delaying the adoption of IBI among smallholders. In the context of developing countries, IBI as a new innovation remains ambiguous for less-educated farmers with less access to information and extension services, particularly during the initial phases of adoption.

However, although ambiguity aversion has a large impact on the timing until farmers start to use IBI, it tends to have less impact on their subsequent dropout or persistent adoption decisions. This varying effect overtime is due to the fact that learning reduces ambiguity, so increased experience in adoption of IBI diminishes the effect of ambiguity aversion overtime. Furthermore, our results suggest that risk aversion does play an important role in IBI adoption decision. We find that risk aversion speeds-up rather than delays the adoption of IBI. This is in contrast with most of the previous empirical tests on the role of risk aversion on technology adoption. The difference can be due to the fact that most empirical literature so far has been considering the whole uncertainty as risk in their analysis, without having a split look at risk and ambiguity that sums up to uncertainty. Our setting directly defines risk as the down-side production risk that smallholders often face due to stochastic drought or seasonality-based variations. Since farmers are averse to production risk, most of them can be fundamentally willing to insure such risk using the IBI contract. However, since there is basis risk in IBI contract design, these farmers can remain ambiguous about whether the contract accurately reveals their actual loss realizations. This shows us that there is interplay between risk and ambiguity aversion in IBI uptake decisions of smallholders. Our analysis reveals that though risk-averse smallholders are willing to buy IBI in order to hedge their production risk, their actual uptake is not effective due to the ambiguity surrounding the validity of the contract to payout in the future. Ambiguity aversion thus dictates uptake decisions of smallholders in a sort of dominance effect. The effect of ambiguity aversion on IBI adoption however diminishes overtime, because farmers' learning and experience in adoption gradually clarifies their ambiguity regarding the nature of the contract. In addition, overtime improvement in the design of IBI like improvement in IBI design that inherently reduces basis risk, helps to reduce the ambiguity surrounding the validity of IBI contract overtime. 


\subsection{Relaxing liquidity constraints increases uptake of weather insurance}

Farmers in developing countries face liquidity constraints due to time-inconsistency between the insurance sells windows and their cash inflow periods. To overcome the problems of intertemporal and spatial adverse selections, insurance firms sell IBIs during specific months preceding the rainy seasons. These months often coincide with the periods when farmers make investments in productivity enhancing inputs like chemical fertilizer and improved seed varieties. Hence, upfront premium payment can become an additional cash outflow that farmers for the time being cannot afford. However, farmers can afford insurance premiums immediately after harvest. We thus observe that there is a liquidity problem due to time inconsistency that limits the uptake of IBI among smallholders in the study area. As reported in Chapter 3, we designed an innovative insurance intervention to overcome this problem. We organized a randomized controlled trial (RCT) experiment involving 8,579 farmers. This experiment has three innovative features. First, parallel to the conventional IBI that requires upfront premium payment we developed an insurance product that postpones premium payments towards shortly after harvest. We called this IOU insurance. Hence, the experiment allows a random subsample of farmers to pay the premium after harvest. Second, we created a customary marketing channel through Iddirs parallel to the conventional statutory channel through cooperatives. Third, the experiment allows a random subsample of households to sign binding contract to overcome the potential strategic defaults associated with repayment of the postponed premium for IOU. From the experimental results we learned that the uptake of the conventional IBI marketed through the statutory channel (cooperatives) is very low (i.e. about $8 \%$ of the potential demand). Overcoming liquidity constraints through the IOU significantly increases uptake controlling for the effects of intervention channel and strategic defaults. Our pilot shows that both marketing via Iddirs and dealing with liquidity constraints via IOUs enhance uptake. However, in isolation, both interventions do not seem to be sufficient: the increase in uptake due to the marketing intervention alone is not significant, while the IOU intervention may be troubled by defaults. Rather, a combination of the two interventions: an IOU with a binding contract, marketed via Iddirs lead to a significant increase in uptake (from $8 \%$ to uptake rates above $30 \%$ ) without default problems. 


\subsection{Trust holds the key in weather insurance uptake decisions}

In Chapter 3 we also test the extent to which overcoming trust problems can enhance the uptake of IBIs using our RCT experiment. Specifically, we create exogenous variation in the marketing channel of IBI to build trust. We marketed the IBI product to farmers through Iddirs. Iddirs in Ethiopia function on principles of reciprocity and altruism, bearing important trustworthiness at the public. In the conventional way, the standard insurance product was marketed and sold via cooperatives. Uptake of the standard product, however, turned out to be very low. The innovative IOU product sold through Iddirs increased uptake enormously as compared with selling the same product through the conventional cooperative channel. Hence, both marketing via Iddirs and dealing with liquidity constraints using IOUs largely enhances uptake. This shows that though some technologies are promising, poor promotion and extension services greatly decrease their uptake. In developing countries, technology adoption decisions are usually channelled through statutory channels. Due to the usual bureaucracy and other drawbacks such channels were not effective. As a result, farmers do not trust information flows through such channels for technology adoption. In this study, we learnt that selling insurance technologies through socially-trusted institutions or Iddirs helps to overcome this problem. The uptake of the innovative IBI channelled through Iddirs is the highest in our experiment revealing that innovative promotion and extension is equally important as offering innovative products or technologies. In essence, this chapter also clarifies that coaxing the market-based weather index insurances with the predominant social insurances enhances the overall uptake, which implies that the two mechanisms are complementary to each other.

\subsection{Contracts reduce strategic defaults in interlinked insurance-credit intervention}

As the IOU may lead to default problems, we also test in Chapter 3 whether this is the case, and we probe whether defaults can be reduced by using binding contracts. This binding contract is a written agreement between smallholders and the insurance firm in a way that a court can impose in case one of the parties attempt to negate on the promise. The objective of our intervention was to improve uptake of the weather insurance product without inducing (strategic) defaults. The experiment specifically created exogenous variations in the type of insurance products, the insurance marketing channel and binding contract. Our results 
revealed that IOU insurance with a binding contract or a joint liability contract, marketed via Iddirs enhances uptake of IBI considerably without serious default problems.

\subsection{Payout under basis risk}

Payout is the direct economic return for the insured. In IBI, payout remains a function of a specific weather variable like rainfall or NDVI. In low income countries where weather infrastructure is poorly developed and terrestrial weather stations are sparse, the discrepancy between index-based computed losses and farm-level actual losses is high. In other words, there exists a high level of basis risk as an imperfect correlation between indexed and real losses. This misleads computations of payouts that farmers are entitled to be paid. Payout can be higher or lower based on the nature of basis risk. During a good weather condition, an upward basis risk with false positive probability (FPP) can cause the index to trigger payout though the insured did not incur losses. This can unfairly awards the farmer with the double bonanza of a good harvest and an insurance payment. Contrarily, during unfavourable weather, payout computation can suffer from a downside basis risk with false negative probability (FNP) that triggers an index of no payout though the insured incurred significant loss due to covariate risk. IBI contracts with high levels of basis risk thus function like a lottery than insurance. Farmers can earn either higher or lower than the expected payout, and basis risk functions like a double-edged sword in determining the amount of payouts.

The adverse consequences of downside basis risk in reducing the amount of payouts in IBI are remarkable. For instance, where credit and insurance coexist in rural areas, inappropriate payouts due to basis risk can induce defaults in two ways. First, smallholders can be unable to repay loans due to cash outflow for premium payment and yield loss due to weather risk. Second, decreased welfare level due to cash outflow combined with expected but not realized payout can induce loan defaulting behaviour. Poor farmers remain behaviourally sensitive to the particular double penalty that once ruined their minimum welfare. When insurance is interlinked with credit, such discouragement can even lead farmers to collectively collude and strategically default on loan repayments. In Chapter 3 we find that defaults are concentrated in a very small number of Iddirs, indicating that members of these Iddirs were coordinated on defaults since they expected but did not earn payouts as a consequence of basis risk. But coordination on defaults threatens the feasibility of credit interlinked insurances. It is evident 
from group-based lending that the potential to use social collateral is often undermined by the behaviour of borrowers who collectively collude against the lender. Hence, under basis risk, IBI provides probabilistic payouts that can lead to distrust and strategic collective defaults. However, as discussed in Chapter 3, using socially-trusted institutions to promote insurance helps to reduce strategic defaults through the mechanisms of peer pressure, monitoring the behavior of adopters and enforcing repayment.

\subsection{Crowding-in credit through insurance}

As an extension to Chapter 3 which aims to insure farmers through a postponed premium, one of the areas where we are interested to see the impact of IBI adoption is whether the adoption of IBI improves access to financial markets and reduces credit rationing. It is evident that credit rationing prevails among households in developing countries. Among smallholders that dominate the developing world, the condition of credit rationing is exacerbated due to their repetitive exposure to covariate weather shocks (Udry 1990; McIntosh et al. 2013). In the absence of formal insurance, such shocks simultaneously overwhelm all households and their community-based mutual risk-sharing arrangements (Genicot and Ray 2003; Alger and Weibull 2010; Barrett 2011; Ambrus et al. 2014). Lenders consider smallholders under covariate shocks as non-credit worthy due to loan default potential in the current period and depleted wealth for collateralization in subsequent periods. This aggravates credit rationing behaviour and prohibits farmers from undertaking risky but remunerative agricultural practices like use of improved seed varieties or application of chemical fertilizer. Since weather risk is the core source of credit rationing in lending for smallholders, innovative weather risk hedging instruments can be integrated with credit facilities to (partially) overcome production risk and rural financial market failure. Integrating IBIs with credit and agricultural technology is appealing, and justified in various recent studies for mutual benefit of borrowers and lenders (Giné and Yang 2009; Hill et al. 2013; McIntosh et al. 2013). The benefit to the smallholder is both access to loan in order to acquire inputs that enhance productivity, and access to insurance to hedge down-side production risk. Lenders also benefit since default risk from lending for insured borrowers is lower than the risk to lend for uninsured ones because of their difference in ability to repay loan. This incentive can motivate lenders to enter markets with minimized default risk that would otherwise cannot be 
anticipated when the production risk that leads to default risk is not insured (McIntosh et al. 2013). Thus, IBI crowds-in credit supply and as farmers need such arrangement; it also crowds-in credit demand. At its face value, this win-win strategy forms a financial environment where credit and IBI complementarily reinforce (crowd-in) each other, and where both the borrower and the lender can remain better off.

However, the fact that IBI contracts sometimes remain invalid to trigger payout even when the insured incurs significant yield losses due to weather risk can cause loan defaults. Consequently, lenders who take this loan default potential into account may limit their credit supply to areas where IBI is implemented. In a natural setting in developing countries where IBI joined the long standing tinny rural credit structures that operate with limited effectiveness, whether the mutual functioning of credit and insurance overtime complement (crowd-in), or substitute (crowd-out) each other is an empirical issue and unknown in advance a prior. This is also a timely and relevant issue for policy aimed at improving the functioning of rural financial markets through credit and insurance interlinkage.

In Chapter 4, we have empirically tested this debate. Specifically, we tested the effect of IBI uptake on credit rationing using data from a drought-prone area in the Rift Valley zone of Ethiopia. In this area, IBI was implemented in selective pilot districts to manage income shocks that households face due to climate change induced drought. Various branches of commercial banks and microfinance institutions have been operating in this area. But no empirical study that investigates how the adoption of IBI has affected the credit rationing behaviour of these institutions was undertaken. We used Direct Elicitation Method (DEM) in line with Boucher et al. (2009) to identify credit rationing status and show that $38.2 \%$ of the sample households were credit constrained. We next show that uptake of IBI reduces credit rationing.

We used an identification strategy, in line with a cross-sectional double difference method, to control for potential selection biases by forecasting potential insurance adopters in the control region. This enables us to draw some conclusions regarding the causal relationship between adopting index-based insurance and credit rationing. We differentiate supply-side and demand-side forms of credit rationing, and identified that IBI reduces supply-side (quantity) 
rationing, while the impact on demand-side credit rationing is statistically insignificant. Thus adoption of IBI could enhance smallholders' access to credit. It also provides mutual benefits to both farmers (borrowers) and lenders. The benefits to the smallholder are two-fold. First, the alleviation of supply-side constraints can enable famers to acquire inputs and enhance productivity. Second, as access to credit overcomes liquidity constraints of farmers, this helps them to employ other risk management strategies to hedge against down-side production risk. Lenders can also benefit from lending to the insured farmers as the default risk of lending to insured borrowers tends to be lower. Hence, the complementary nature of IBI and credit also make borrowers and lenders better off in this context. We accordingly foresee that this study encourages new research that seeks to test whether our results also hold in other settings and are also robust to other identification strategies, such as the use of randomized controlled trials.

\subsection{Impacts of IBI adoption on household welfare: consumption and investment}

In this thesis, we estimated the impact of IBI adoption on household production and consumption behaviour. In Chapter 5, we evaluate the impact of IBI adoption on two categories of welfare indicators: household consumption and investment in high-risk highreturn inputs. We focussed on the issue that in impact evaluation studies, self-selection and program placement biases often complicate causal attributions of welfare improvements to rural interventions. We used a two-round panel data collected from smallholders in the Rift Valley zone of south-eastern Ethiopia. IBI adoption in the area has lasted four years before we undertake the study, so we expected that lasting welfare effects can be established. Consistently, our results indicated that adoption of IBI has significantly increased both investment in high-risk high-return inputs and per capita household consumption expenditure. Using a random effect model with flexible specification that takes the frequency of adoption into account, we further understood that investment in high-risk high-return inputs as well as

per capita household consumption is increased with the frequency of IBI adoption. In addition, we see evidence that one-time adoption had no significant impact on these outcome indicators, but repeated cycles of adoption do matter to achieve significant welfare impacts from IBI adoption. 


\subsection{General conclusion}

Shocks matter. Shocks that smallholders face are often caused by natural variations and exacerbated by climate change. Adverse welfare consequences of shocks can drive smallholders to lapse in consumption fluctuation and food insecurity situations, and then gradually lead them trapped in poverty. In the face of climate shocks, an alternative to emergency (food) aid is reliable interventions that can minimize farmers' risk and prevent their slide into poverty traps. This thesis focuses on the strategic ways of managing risks using innovative insurance markets. Insurance is the policy option to manage risks. IBI is an innovative pro-poor climate risk management strategy that bears the potential to overcome the problems associated with information asymmetry that often plagues the development of rural financial markets. In recent years, interest in the study of index insurance in rural areas of the developing world has increased. To date, much of the weather insurance research in lowincome countries has focused on reporting the low uptake of index insurance among smallholders. This thesis provides overview of the potential effects of shocks on welfare, and puts in line the systematic ways of managing the adverse effects of shocks through implementing innovative insurance mechanisms. In particular, we undertake analysis of the micro-level demand, impact and overall effectiveness of innovative insurance interventions designed for smallholders. We start from the evidence that IBI can have an immense potential for the management of risks that smallholders face. But the demand is sluggish, the uptake is low and as a result the full potential welfare gains were not yet derived from IBIs. There is limited understanding on how behavioural, socio-economic and institutional factors influence the uptake of IBI.

We address this important gap in the literature while simultaneously making an important contribution to disentangling the ex-ante and ex-post welfare effects of IBI in terms of households' investment in high-risk high-return agricultural inputs, consumption and access to financial markets. We use lab-in-the-field experiments, randomized controlled trial and household panel data collected between 2015 and 2017 to identify the main impediments of IBI adoption, to experimentally test how to enhance the uptake of IBI among smallholders and to detect the causal effect of IBI on household welfare. Using lab-in-the field experiments combined with household survey, we separate out the effects of ambiguity aversion behaviour 
from the effects of risk aversion in influencing the uptake of IBI. We find that ambiguity aversion behaviour delays while risk aversion behaviour speeds-up the adoption of IBI. We also find evidence that the effect of ambiguity aversion on IBI adoption diminishes overtime. The fact that the effect of ambiguity aversion on IBI adoption diminishes overtime suggests the importance of learning mechanisms such as training in shaping the behaviour and capacity of individual smallholders to manage uncertainty in adoption of new technologies. Using the randomized controlled trial we delineated that an innovative IBI that overcomes the liquidity constraints of households by postponing the premium payment enormously increases the uptake of IBI. There is even more increase in uptake when the innovative IOU insurance is offered through the customary social insurance channel Iddirs as compared with offering the same product through the statutory cooperative channel. This marginal increase in uptake due to the customary channel is attributed to the altruistic trust that smallholders have on their Iddirs. The high acceptance of the IBI through Iddirs also implies the win-win advantage from coaxing the commercial insurance with the social insurance. We also tested the relevance of binding contracts to enforce the repayment of the postponed IOU premium. This is especially important to minimize the strategic default. We find that the binding social contract in Iddir membership as well as the legal contract helps to reinforce repayment without jeopardizing the increase in uptake of the IBI.

To understand weather the adoption of IBI enhances households' access to financial markets; we used an innovative identification strategy in line with the cross-sectional difference-indifference method. We find that adoption of IBI reduces credit supply rationing among smallholders. The main policy implication from this result is that IBI and credit can be complementary rural financial services in developing countries. We measured the ex-ante welfare impacts of IBI using the household panel data and the difference-in-difference approach. Specifically, we evaluated the impact of IBI adoption on household investment in three high-risk high-return agricultural inputs: fertilizer, improved seed variety and pesticide/herbicide. We find that IBI coverage has a strongly positive and statistically significant effect on household investment in chemical fertilizer and improved seed varieties. We also find evidence of a statistically significant positive effect of IBI adoption on household consumption. 


\subsection{Policy implications}

We can draw several policy implications from the different studies included in this thesis. The first is the need to distinguish between risk and ambiguity aversion behaviour of households in analysis of technology adoption decision. Second, the effects of risk and ambiguity aversion on technology adoption can vary over time. These implications underscore the need for future studies to theoretically distinguish and empirically test the relative relevance of the effect of risk and ambiguity aversion in technology adoption decisions. Similarly, though IBIs were priced at relatively low prices, farmers face liquidity problems because during the sowing period smallholders were largely engaged in purchase of productivity enhancing inputs like improved seed varieties and chemical fertilizer. However, after harvest farmers' liquidity level obviously helps them to repay insurance premiums. Hence, since smallholders face problems of time-inconsistency in their cash flows and insurance purchase decisions, policy instruments that overcome such liquidity constraints due to time inconsistency like the IOU insurance we designed should be used in the next generations of drought insurances. An important implication of these results for microinsurance firms like OIC is that IBI-adopter households should be encouraged not only to participate in adoption once, but also to sustain their uptake by renewing the IBI policy, and by remaining for longer periods in adoption phase in order to realize the full potentials of welfare gains. The policy implication is that customized financial products to handle risk must be correspondingly developed based on rigorous experimentation and impact analysis. Our research also has methodological implications in designing studies related to the adoption of rural technologies in future. Combining experimental methods like the lab-in-the-field experiments and randomized controlled trial techniques to measure variables that are otherwise difficult to identify like risk and ambiguity aversion with survey methods helps to better understand the role of the behaviour of decision making units. The effect of IBI adoption on household welfare can be multidimensional and may not be fully captured by just a single household welfare outcome indicator. Second, the effect of IBI adoption lasts longer than one or two years. It takes time before the effect of IBI adoption on consumption or investment in high-risk high-return inputs is fully materialized. Therefore, impact estimates that rely on a single household welfare indicator and focus only on one cycle of IBI adoption may underestimate the potential welfare gains that can be achieved overtime. 


\subsection{Limitations}

In identifying the determinants of uptake, although we considered many variables, there is still a potential for omitted variables. For instance, we might think that people who are more wellconnected in social networks may more likely adopt IBI. But we do not have data on social networks. Thus, since we were not duly emphasized on the role of social networks, experimental data on social networks would have further strengthened our findings. The way we measured trust is also not based on experimental approaches or trust games. Hence, future studies can closely assess the effects of trust on adoption of IBI using a standardized experiment based trust measures like the use of trust games. Further, in conducting the RCT with the innovative IBI product that postpones the upfront premium payment, we did not test the efficacy of directly selling the product to Iddirs and the effect of post-payout distributions of earnings from insurance among the Iddir members. Iddirs pool risks through compensating losses in kind or cash from a common fund. Within Iddirs, cohesive groups and sub-groups have strong risksharing ties that may help to internalize the problems of basis risk in IBIs. Thus, it is imperative to experiment the uptake and impacts of selling IBIs to Iddirs, and understand how it aligns with some earlier findings that formal insurances crowd-out risk-sharing functions of informal insurances. In assessing the impacts of IBI on household welfare, we used only a two-year tworound panel data. This seems to be a promising avenue for future investigations on the welfare impacts of IBIs with a larger panel dataset in terms of time as well as units of observations.

\subsection{The way forward}

IBI is an institutional innovation with immense economic potential, but it faces the challenge of low uptake. This raises the issue of identifying the potential avenues for sustainable scale up. We examined the main determinants of low uptake, and experimentally explored the innovative ways of enhancing uptake in a sustainable way. For the way forward, this thesis puts the following directions. The primary issue is strategic ways of reducing basis risk. The effectiveness of offering IBI for smallholders principally hinges on innovation ability to improve the quality of the insurance by reducing the underlying basis risk. We provided a new evidence of behavioural economics that due to strong ambiguity aversion behaviour among smallholders, basis risk is even more important in limiting the uptake of IBI than predicted by 
conventional expected utility theory. Future efforts should focus on multiple institutional, contractual and technological innovations as important advances that can reduce basis risk. The second most important agenda is promoting research on behaviour. IBI uptake largely relies on household behaviour. Still there remain a vast category of behavioural aspects of insurance demand that need to be better understood. Issues including low willingness to pay for IBI even at premiums close to zero, buyers' fatalism to take-up new technology, unrealistic trust in others, recency bias in assessing risk, time inconsistency and prospect theory should be well understood (Kahneman 1979; List 2004). The third is designing innovative products. IBI products should be innovative to overcome the problems of liquidity, understandabaility and trust. Insurance products should also be developed in relation to the behaviour of the smallholders. Coaxing market based IBIs with predominant social insurances is also important. Interlinking IBI with credit and using binding contracts to enforce repayment are also important elements of IBI innovation. Future research on the welfare impacts of weather insurance must focus on more robust specifications that incorporate temporal as well as multidimensional effects of IBI adoption on household welfare. First, measuring the impact of IBIs should be robust and complete. Our study shows that IBIs have both ex-ante benefits that insured households realize from prudential investment in production inputs without payouts, and ex-post benefits in terms of payouts after loss realizations. Future studies should undertake more robust theoretical modelling and empirical evaluations of the impact of IBIs from multiple perspectives. This remains very important in current arena when large numbers of rural producers and entrepreneurs inherently face challenges of managing uncertainty in an increasingly volatile global economy. 


\section{References}

Ahlin, C., \& Jiang, N. (2008). Can micro-credit bring development? Journal of Development Economics, 86(1), 1-21.

Akay, A., Martinsson, P., Medhin, H., \& Trautmann, S. T. (2012). Attitudes toward uncertainty among the poor: an experiment in rural Ethiopia. Theory and Decision, 73(3), 453-464.

Alger, I., \& Weibull, J. W. (2010). Kinship, incentives, and evolution. American Economic Review, 100(4), 1725-58.

Ambrus, A., Mobius, M., \& Szeidl, A. (2014). Consumption risk-sharing in social networks. American Economic Review, 104(1), 149-82.

Andersen, S., Harrison, G. W., Lau, M. I., \& Rutström, E. E. (2006). Elicitation using multiple price list formats. Experimental Economics, 9(4), 383-405.

Angelucci, M., De Giorgi, G., Rangel, M. A., \& Rasul, I. (2010). Family networks and school enrolment: Evidence from a randomized social experiment. Journal of Public Economics, 94(3-4), 197-221.

Aredo, D. (1993). The Iddir: A study of an indigenous informal financial institution in Ethiopia. Savings and Development, 77-90.

Aredo, D. (2010). The Iddir: An informal insurance arrangement in Ethiopia. Savings and Development, 53-72.

Armendáriz, B., \& Morduch, J. (2010). The economics of microfinance. MIT press.

Arnott, R., \& Stiglitz, J. E. (1991). Moral hazard and nonmarket institutions: Dysfunctional crowding out of peer monitoring? American Economic Review, 179-190.

Ashraf, Nava, Dean S. Karlan, and Wesley Yin (2006). "Tying Odysseus to the Mast: Evidence from a Commitment Savings Product in the Philippines. Quarterly Journal of Economics.

Baland, J. M., Guirkinger, C., \& Mali, C. (2011). Pretending to be poor: Borrowing to escape forced solidarity in Cameroon. Economic Development and Cultural Change, 60(1), 116.

Banerjee, A. V. (2000). The Two Poverties. Nordic Journal of Political Economy, 26(2), 129141. 
Banerjee, A. V., \& Duflo, E. (2009). The experimental approach to development economics. Annual Review of Resource Economics, 1(1), 151-178.

Banerjee, A. V., Banerjee, A., \& Duflo, E. (2011). Poor economics: A radical rethinking of the way to fight global poverty. Public Affairs.

Bardhan, P., Bowles, S., \& Gintis, H. (2000). Wealth inequality, wealth constraints and economic performance. Handbook of Income Distribution, 1, 541-603.

Barham, B. L., Chavas, J. P., Fitz, D., Salas, V. R., \& Schechter, L. (2014). The roles of risk and ambiguity in technology adoption. Journal of Economic Behavior \& Organization, 97, 204-218.

Barnett, B. J., Barrett, C. B., \& Skees, J. R. (2008). Poverty traps and index-based risk transfer products. World Development, 36(10), 1766-1785.

Barrett, C. B. (2011). Covariate catastrophic risk management in the developing world: Discussion. American Journal of Agricultural Economics, 93(2), 512-513.

Barrett, C. B., Christian, P., \& Shiferaw, B. A. (2017). The structural transformation of African agriculture and rural spaces: introduction to a special section. Agricultural Economics, $48(\mathrm{~S} 1), 5-10$.

Becker, G. S., \& Mulligan, C. B. (1997). The endogenous determination of time preference. The Quarterly Journal of Economics, 112(3), 729-758.

Becker, S. W., \& Brownson, F. O. (1964). What price ambiguity? Or the role of ambiguity in decision-making. Journal of Political Economy, 72(1), 62-73.

Beegle, K., Dehejia, R. H., \& Gatti, R. (2003). Child labor, income shocks, and access to credit. The World Bank.

Belissa (2018d). Does Index-based insurance improve household welfare? Empirical evidence based on panel data in south-eastern Ethiopia. Working paper.

Belissa, Bulte, Cecchi, Gangopadhyay and Lensink (2018b). Trust, liquidity constraints and the adoption of weather insurance: A randomized controlled trial in Ethiopia. Under review, Journal of Development Economics.

Belissa, Lensink and Asseldonk (2018a). Risk and ambiguity aversion behaviour in index-based insurance uptake decision: Experimental evidence from Ethiopia. Under review, Journal of Economic Behaviour and Organization. 
Belissa, Lensink and Winkel $\left(2018_{\mathrm{c}}\right)$. Effects of Index Insurance on Demand and Supply of Credit: Evidence from Ethiopia. American Journal of Agricultural Economics revised and submitted.

Berhane, G., \& Gardebroek, C. (2011). Does microfinance reduce rural poverty? Evidence based on household panel data from northern Ethiopia. American Journal of Agricultural Economics, 93(1), 43-55.

Bernhard, H., Fischbacher, U., \& Fehr, E. (2006). Parochial altruism in humans. Nature, 442(7105), 912.

Bernheim, B. D., \& Whinston, M. D. (1998). Incomplete contracts and strategic ambiguity. American Economic Review, 902-932.

Besanko, D., \& Thakor, A. V. (1987). Competitive equilibrium in the credit market under asymmetric information. Journal of Economic Theory, 42(1), 167-182.

Besley, T. (1995). Savings, credit and insurance. Handbook of Development Economics, 3, $2123-$ 2207.

Besley, T., \& Coate, S. (1995). Group lending, repayment incentives and social collateral. Journal of Development Economics, 46(1), 1-18.

Bester, H. (1985). Screening vs. rationing in credit markets with imperfect information. American Economic Review, 75(4), 850-855.

Bester, H. (1987). The role of collateral in credit markets with imperfect information. European Economic Review, 31(4), 887-899.

Biazin, B., \& Sterk, G. (2013). Drought vulnerability drives land-use and land cover changes in the Rift Valley dry lands of Ethiopia. Agriculture, Ecosystems \& Environment, 164, 100113.

Binswanger, H. P. (1980). Attitudes toward risk: Experimental measurement in rural India. American Journal of Agricultural Economics, 62(3), 395-407.

Binswanger, H. P., \& Rosenzweig, M. R. (1986). Behavioural and material determinants of production relations in agriculture. Journal of Development Studies, 22(3), 503-539.

Binswanger-Mkhize, H. P. (2012). Is there too much hype about index-based agricultural insurance? Journal of Development Studies, 48(2), 187-200. 
Borghans, L., Heckman, J. J., Golsteyn, B. H., \& Meijers, H. (2009). Gender differences in risk aversion and ambiguity aversion. Journal of the European Economic Association, 7(2-3), $649-658$.

Boucher, S. R., Carter, M. R., \& Guirkinger, C. (2008). Risk rationing and wealth effects in credit markets: Theory and implications for agricultural development. American Journal of Agricultural Economics, 90(2), 409-423.

Boucher, S. R., Guirkinger, C., \& Trivelli, C. (2009). Direct elicitation of credit constraints: Conceptual and practical issues with an application to Peruvian agriculture. Economic Development and Cultural Change, 57(4), 609-640.

Boucher, S., \& Guirkinger, C. (2007). Risk, wealth, and sectoral choice in rural credit markets. American Journal of Agricultural Economics, 89(4), 991-1004.

Brick, K., Visser, M., \& Burns, J. (2012). Risk aversion: Experimental evidence from South African fishing communities. American Journal of Agricultural Economics, 94(1), 133152.

Bryan, G. (2010). Ambiguity and insurance. Unpublished manuscript.

Budescu, D. V., \& Fischer, I. (2001). The same but different: an empirical investigation of the reducibility principle. Journal of Behavioural Decision Making, 14(3), 187-206.

Cai, H., Chen, Y., Fang, H., \& Zhou, L. A. (2009). Microinsurance, trust and economic development: Evidence from a randomized natural field experiment (No. w15396). National Bureau of Economic Research.

Cai, H., Chen, Y., Fang, H., \& Zhou, L. A. (2015). The effect of microinsurance on economic activities: evidence from a randomized field experiment. Review of Economics and Statistics, 97(2), 287-300.

Cai, J. (2016). The impact of insurance provision on household production and financial decisions. American Economic Journal: Economic Policy, 8(2), 44-88.

Cai, J., \& Song, C. (2017). Do disaster experience and knowledge affect insurance take-up decisions. Journal of Development Economics, 124, 83-94.

Cai, J., De Janvry, A., \& Sadoulet, E. (2015). Social networks and the decision to insure. American Economic Journal: Applied Economics, 7(2), 81-108.

Camerer, C., \& Weber, M. (1992). Recent developments in modeling preferences: Uncertainty and ambiguity. Journal of Risk and Uncertainty, 5(4), 325-370. 
Cameron, A. C., Gelbach, J. B., \& Miller, D. L. (2008). Bootstrap-based improvements for inference with clustered errors. The Review of Economics and Statistics, 90(3), 414-427.

Cardenas, J. C., \& Carpenter, J. (2008). Behavioural development economics: Lessons from field labs in the developing world. The Journal of Development Studies, 44(3), 311-338.

Carlin, P. S. (1992). Violations of the reduction and independence axioms in Allais-type and common-ratio effect experiments. Journal of Economic Behavior \& Organization, 19(2), 213-235.

Carlsson, F., Martinsson, P., Qin, P., \& Sutter, M. (2013). The influence of spouses on household decision making under risk: an experiment in rural China. Experimental Economics, 16(3), 383-401.

Carter, M. R. (1997). Environment, technology, and the social articulation of risk in West African agriculture. Economic Development and Cultural Change, 45(3), 557-590.

Carter, M. R., Cheng, L., \& Sarris, A. (2016). Where and how index insurance can boost the adoption of improved agricultural technologies. Journal of Development Economics, 118 , 59-71.

Carter, M., de Janvry, A., Sadoulet, E., \& Sarris, A. (2014). Index-based weather insurance for developing countries: A review of evidence and a set of propositions for up-scaling. Development Policies working paper, 111.

Carter, M., de Janvry, A., Sadoulet, E., \& Sarris, A. (2017). Index insurance for developing country agriculture: a reassessment. Annual Review of Resource Economics, 9, 421-438.

Carter, M., Elabed, G., \& Serfilippi, E. (2015). Behavioral economic insights on index insurance design. Agricultural Finance Review, 75(1), 8-18.

Casaburi, L., \& Willis, J. (2019). Time vs. state in insurance: Experimental evidence from contract farming in Kenya. American Economic Review. Forthcoming.

Caselli, F. (1997). Rural labor and credit markets. Journal of Development Economics, 54(2), 235-260.

Cassar, A., Healy, A., \& Von Kessler, C. (2017). Trust, risk, and time preferences after a natural disaster: experimental evidence from Thailand. World Development, 94, 90-105.

Cecchi, F., Duchoslav, J., \& Bulte, E. (2016). Formal insurance and the dynamics of social capital: Experimental evidence from Uganda. Journal of African Economies, 25(3), 418438. 
Chantarat, S. (2009). Pro-poor risk management: essays on the economics of index-based risk transfer products. $\mathrm{PhD}$ dissertation. University of California at Davis.

Chantarat, S., Mude, A. G., Barrett, C. B., \& Carter, M. R. (2013). Designing index-based livestock insurance for managing asset risk in northern Kenya. Journal of Risk and Insurance, 80(1), 205-237.

Charness, G., \& Genicot, G. (2009). Informal risk sharing in an infinite-horizon experiment. The Economic Journal, 119(537), 796-825.

Cheng, L. (2014). The impact of index insurance on borrower's moral hazard behavior in rural credit markets. Working Paper. Department of Agricultural and Resource Economics. University of California, Davis.

Chetty, R., \& Szeidl, A. (2007). Consumption commitments and risk preferences. The Quarterly Journal of Economics, 122(2), 831-877.

Christiaensen, L., Demery, L., \& Kuhl, J. (2011). The (evolving) role of agriculture in poverty reduction-An empirical perspective. Journal of Development Economics, 96(2), 239254.

Clarke, D. J. (2016). A theory of rational demand for index insurance. American Economic Journal: Microeconomics, 8(1), 283-306.

Clarke, D. J., Mahul, O., \& Verma, N. (2012). Index based crop insurance product design and ratemaking: The case of modified NAIS in India. The World Bank.

Clarke, D., \& Dercon, S. (2009). Insurance, credit and safety nets for the poor in a world of risk. United Nations, Department of Economics and Social Affairs. Working Paper 81.

Coate, S., \& Ravallion, M. (1993). Reciprocity without commitment: Characterization and performance of informal insurance arrangements. Journal of Development Economics, $40(1), 1-24$.

Cole, S. (2012). The effectiveness of index-based micro-insurance in helping smallholders manage weather-related risks.

Cole, S., Giné, X., Tobacman, J., Topalova, P., Townsend, R., \& Vickery, J. (2013). Barriers to household risk management: Evidence from India. American Economic Journal: Applied Economics, 5(1), 104-35.

Cole, S., Stein, D., \& Tobacman, J. (2011). What is rainfall index insurance worth? A comparison of valuation techniques. Mimeo. 
Coleman, B. E. (1999). The impact of group lending in Northeast Thailand. Journal of Development Economics, 60(1), 105-141.

Coleman, B. E. (2006). Microfinance in Northeast Thailand: Who benefits and how much? World Development, 34(9), 1612-1638.

Conning, J., \& Udry, C. (2007). Rural financial markets in developing countries. Handbook of Agricultural Economics, 3, 2857-2908.

Copestake, J., Bhalotra, S., \& Johnson, S. (2001). Assessing the impact of microcredit: A Zambian case study. Journal of Development Studies, 37(4), 81-100.

Couture, P. (2008). Informed consent in social science. Science, 322(5902), 672-672.

Cummins, J. D., Lalonde, D., \& Phillips, R. D. (2004). The basis risk of catastrophic-loss index securities. Journal of Financial Economics, 71(1), 77-111.

De Bock, O., \& Ontiveros, D. (2013). Literature review on the impact of microinsurance. Microinsurance Innovation Facility, Research Paper, (35).

De Janvry, A., Dequiedt, V., \& Sadoulet, E. (2014). The demand for insurance against common shocks. Journal of Development Economics, 106, 227-238.

Deaton, A. (1992). Household saving in LDCs: Credit markets, insurance and welfare. The Scandinavian Journal of Economics, 253-273.

Deaton, A. (2010). Instruments, randomization, and learning about development. Journal of Economic Literature, 48(2), 424-55.

DellaVigna, S., List, J. A., \& Malmendier, U. (2012). Testing for altruism and social pressure in charitable giving. The Quarterly Journal of Economics, 127(1), 1-56.

Dercon, S. (1996). Risk, crop choice, and savings: Evidence from Tanzania. Economic Development and Cultural Change, 44(3), 485-513.

Dercon, S. (2004). Growth and shocks: Evidence from rural Ethiopia. Journal of Development Economics, 74(2), 309-329.

Dercon, S. (2008). Fate and fear: Risk and its consequences in Africa. Journal of African Economies, 17(suppl_2), ii97-ii127.

Dercon, S., \& Christiaensen, L. (2011). Consumption risk, technology adoption and poverty traps: Evidence from Ethiopia. Journal of Development Economics, 96(2), 159-173. 
Dercon, S., De Weerdt, J., Bold, T., \& Pankhurst, A. (2006). Group-based funeral insurance in Ethiopia and Tanzania. World Development, 34(4), 685-703.

Dercon, S., Hill, R. V., Clarke, D., Outes-Leon, I., \& Taffesse, A. S. (2014). Offering rainfall insurance to informal insurance groups: Evidence from a field experiment in Ethiopia. Journal of Development Economics, 106, 132-143.

Dercon, S., Hoddinott, J., \& Woldehanna, T. (2005). Shocks and consumption in 15 Ethiopian villages, 1999-2004. Journal of African Economies, 14(4), 559-585.

Dowd, K. (1992). Optimal financial contracts. Oxford Economic Papers, 44(4), 672-693.

Duflo, E, M. Kremer, and J. Robinson.( 2011). Nudging Farmers to Use Fertilizer: Theory and Experimental Evidence from Kenya. American Economic Review, 101 (6): 2350-90.

Duflo, E., Glennerster, R., \& Kremer, M. (2007). Using randomization in development economics research: A toolkit. Handbook of Development Economics, 4, 3895-3962.

Duflo, Esther and Emmanuel Saez (2003). "The Role of Information and Social Interactions in Retirement Plan Decisions: Evidence from a Randomized Experiment," The Quarterly Journal of Economics, 118(3): 815-842.

Elabed, G. \& M. R. Carter (2015). Compound-risk aversion, ambiguity and the willingness to pay for microinsurance. Journal of Economic Behavior \& Organization, 118, 150-166.

Elabed, G., \& Carter, M. (2014). Ex-ante impacts of agricultural insurance: Evidence from a field experiment in Mali. University of California at Davis.

Elabed, G., M. F. Bellemare, M. R. Carter \& C. Guirkinger (2013). Managing basis risk with multiscale index insurance. Agricultural Economics, 44, 419-431.

Ellsberg, D. (1961) Risk, ambiguity, and the Savage axioms. The Quarterly Journal of Economics, 643-669.

Emerick, K., A. de Janvry, E. Sadoulet and M. Dar (2016). Technological innovations, downside risk, and the modernization of agriculture. American Economic Review, 106: 1537-1561

Eswaran, M., \& Kotwal, A. (1990). Implications of credit constraints for risk behaviour in less developed economies. Oxford Economic Papers, 42(2), 473-482.

Fafchamps, M. \& S. Lund (2003). Risk-sharing networks in rural Philippines. Journal of Development Economics, 71, 261-287. 
Fafchamps, M. (2010). Vulnerability, risk management, and agricultural development. African Journal of Agricultural Economics, 5(1), 243-60.

Fafchamps, M., \& Gubert, F. (2007). The formation of risk sharing networks. Journal of Development Economics, 83(2), 326-350.

Fafchamps, Marcel, and John Pender (1997). "Precautionary Saving, Credit Constraints, and Irreversible Investment: Theory and Evidence from Semiarid India." Journal of Business and Economic Statistics, 152(2): 180-194.

Farrin, K. \& M. J. Miranda (2015) A heterogeneous agent model of credit-linked index insurance and farm technology adoption. Journal of Development Economics, 116, 199-211.

Farrin, K., \& Miranda, M. J. (2015). A heterogeneous agent model of credit-linked index insurance and farm technology adoption. Journal of Development Economics, 116, 199211.

Feder, G., Just, R.E., Zilberman, D. (1985). Adoption of agricultural innovations in developing countries: survey. Economic Development and Cultural Change, 33, 255-298.

Fehr, E., \& Fischbacher, U. (2003). The nature of human altruism. Nature, 425(6960), 785.

Foster, A. D., \& Rosenzweig, M. R. (2010). Microeconomics of technology adoption. Annu. Rev. Econ., 2(1), 395-424.

Fox, C.R., Tversky, A. (1995). Ambiguity aversion and comparative ignorance. The Quarterly Journal of Economics, 110, 585-603.

Fox, C.R., Weber, M. (2002). Ambiguity aversion, comparative ignorance, and decision context. Organizational Behavior and Human Decision Processes, 88, 476-498.

Fuchs, A., \& Wolff, H. (2011). Concept and unintended consequences of weather index insurance: the case of Mexico. American Journal of Agricultural Economics, 93(2), 505511.

Genicot, G., \& Ray, D. (2003). Group formation in risk-sharing arrangements. The Review of Economic Studies, 70(1), 87-113.

Gerber, A. S., \& Green, D. P. (2012). Field experiments: Design, analysis, and interpretation. WW Norton.

Ghatak, M. (1999). Group lending, local information and peer selection1. Journal of Development Economics, 60(1), 27-50. 
Ghosh, P., Mookherjee, D., \& Ray, D. (2000). Credit rationing in developing countries: An overview of the theory. Readings in the Theory of Economic Development, 383-401.

Giné, X., \& Yang, D., (2009). Insurance, credit, and technology adoption: Field experimental evidencefrom Malawi. Journal of Development Economics, 89(1), 1-11.

Giné, X., Townsend, R., \& Vickery, J. (2008). Patterns of rainfall insurance participation in rural india. The World Bank Economic Review, 22(3), 539-566.

Glauber, J. W. (2004). Crop insurance reconsidered. American Journal of Agricultural Economics, 86(5), 1179-1195.

Gneezy, U., \& List, J. A. (2006). Putting behavioral economics to work: Testing for gift exchange in labor markets using field experiments. Econometrica, 74(5), 1365-1384.

Gneezy, U., \& Potters, J. (1997). An experiment on risk taking and evaluation periods. The Quarterly Journal of Economics, 112(2), 631-645.

Gneezy, U., Leonard, K. L., \& List, J. A. (2009). Gender differences in competition: Evidence from a matrilineal and a patriarchal society. Econometrica, 77(5), 1637-1664.

Gollier, C. (1994). Insurance and precautionary capital accumulation in a continuous-time model. Journal of Risk and Insurance, 78-95.

Guarcello, L., F. Mealli \& F. C. Rosati (2010). Household vulnerability and child labor: the effect of shocks, credit rationing, and insurance. Journal of Population Economics, 23, 169-198.

Haggblade, S., Hazell, P. B., \& Dorosh, P. A. (2007). Sectoral growth linkages between agriculture and the rural nonfarm economy. Transforming the rural nonfarm economy: Opportunities and threats in the developing world, 141-182.

Harrison, G. W., \& List, J. A. (2004). Field experiments. Journal of Economic literature, 42(4), 1009-1055.

Harrison, G. W., Humphrey, S. J., \& Verschoor, A. (2009). Choice under uncertainty: evidence from Ethiopia, India and Uganda. The Economic Journal, 120(543), 80-104.

Harrison, G. W., List, J. A., \& Towe, C. (2007). Naturally occurring preferences and exogenous laboratory experiments: A case study of risk aversion. Econometrica, 75(2), 433-458.

Harrison, G.W., Lau, M.I., Rutström, E.E. (2009). Risk attitudes, randomization to treatment, and self-selection into experiments. Journal of Economic Behaviour and Organization, $70,498-507$. 
Harrower, S., \& Hoddinott, J. (2005). Consumption smoothing in the zone Lacustre, Mali. Journal of African Economies, 14(4), 489-519.

Hazell, P. B. (1992). The appropriate role of agricultural insurance in developing countries. Journal of International Development, 4(6), 567-581.

Henrich, J., Boyd, R., Bowles, S., Camerer, C., Fehr, E., Gintis, H., \& McElreath, R. (2001). In search of homo economicus: behavioral experiments in 15 small-scale societies. American Economic Review, 91(2), 73-78.

Henrich, J., Ensminger, J., McElreath, R., Barr, A., Barrett, C., Bolyanatz, A., ... \& Lesorogol, C. (2010). Markets, religion, community size, and the evolution of fairness and punishment. Science, 327(5972), 1480-1484.

Henrich, J., Mcelreath, R., Barr, A., Ensminger, J., Barrett, C., Bolyanatz, E., ... \& Gwako, E. (2008). Costly punishment across human societies. Science, 312, 1767-1770.

Hill, R. V., Hoddinott, J., \& Kumar, N. (2013). Adoption of weather-index insurance: Learning from willingness to pay among a panel of households in rural Ethiopia. Agricultural Economics, 44(4-5), 385-398.

Holt, C., and S. Laury (2002). Risk Aversion and Incentive Effects. American Economic Review, $92,1644-1655$.

Hulme, M., R. Doherty, T. Ngara, M. New \& D. Lister (2001). African Climate Change: 19002100. Climate research, 17, 145-168.

Imbens, G. W. (2003). Sensitivity to exogeneity assumptions in program evaluation. American Economic Review, 93(2), 126-132.

Imbens, G. W. (2015). Matching methods in practice: Three examples. Journal of Human Resources, 50(2), 373-419.

Islam, A., \& Maitra, P. (2012). Health shocks and consumption smoothing in rural households: Does microcredit have a role to play? Journal of Development Economics, 97(2), 232243.

Iturrioz, R. (2009). Agricultural insurance (No. E20-77). The World Bank.

Izhakian, Y. \& S. Benninga (2011). The uncertainty premium in an ambiguous economy. The Quarterly Journal of Finance, 1, 323-354.

Jaffee, D. M., \& Russell, T. (1976). Imperfect information, uncertainty, and credit rationing. The Quarterly Journal of Economics, 90(4), 651-666. 
Jalan, J., \& Ravallion, M. (1999). Are the poor less well insured? Evidence on vulnerability to income risk in rural China. Journal of Development Economics, 58(1), 61-81.

Janzen, S. A., \& Carter, M. R. (2013). The impact of microinsurance on consumption smoothing and asset protection: Evidence from a drought in Kenya. University of Califronia at Davis. Working Paper.

Jayachandran, S. (2006). Selling labor low: Wage responses to productivity shocks in developing countries. Journal of Political Economy, 114, 538-575.

Jensen, N. D., Mude, A., \& Barrett, C. B. (2014). How basis risk and spatiotemporal adverse selection influence demand for index insurance: Evidence from northern Kenya. Social Science Research Network. Working Paper, No. 2475187.

John, A. (2008). Homo experimentalis evolves. Science, 321(5886), 207-208.

Just, R. E., Calvin, L., \& Quiggin, J. (1999). Adverse selection in crop insurance: Actuarial and asymmetric information incentives. American Journal of Agricultural Economics, 81(4), 834-849.

Kahneman, D. (1979). Prospect theory: An analysis of decisions under risk. Econometrica, 47, 278.

Karlan, D. S., \& Goldberg, N. (2007). Impact evaluation for microfinance: Review of methodological issues. World Bank, Poverty Reduction and Economic Management, Thematic Group on Poverty Analysis, Monitoring and Impact Evaluation.

Karlan, D., Osei, R., Osei-Akoto, I., \& Udry, C. (2014). Agricultural decisions after relaxing credit and risk constraints. The Quarterly Journal of Economics, 129(2), 597-652.

Karlan, Dean (2005). "Using Experimental Economics to Measure Social Capital and Predict Real Financial Decisions," American Economic Review, 95(5): 1688-1699.

Khandker, S. (2005). Microfinance and Poverty: Evidence Using Panel Data from Bangladesh. World Bank Economic Review, 19(2): 263-286.

Klibanoff, P., Marinacci, M., Mukerji, S. (2005). A smooth model of decision making under ambiguity. Econometrica, 73, 1849-1892.

Kovacevic, R. M., \& Pflug, G. C. (2011). Does insurance help to escape the poverty trap?-A ruin theoretic approach. Journal of Risk and Insurance, 78(4), 1003-1028.

Kumar, K. B., \& Matsusaka, J. G. (2009). From families to formal contracts: An approach to development. Journal of Development Economics, 90(1), 106-119. 
Laibson D. (1997). "Golden Eggs and Hyperbolic Discounting," Quarterly Journal of Economics, 62: 443-478.

Lamb, R. L. (2003). Fertilizer use, risk, and off-farm labor markets in the semi-arid tropics of India. American Journal of Agricultural Economics, 85(2), 359-371.

Lensink, R., \& Sterken, E. (2001). Asymmetric information, option to wait to invest and the optimal level of investment. Journal of Public Economics, 79(2), 365-374.

Lensink, R., \& Sterken, E. (2002). The option to wait to invest and equilibrium credit rationing. Journal of Money, Credit, and Banking, 34(1), 221-225.

Levitt, S. D., \& List, J. A. (2008). Homo economicus evolves. Science, 319(5865), 909-910.

List, J. A. (2004). Neoclassical theory versus prospect theory: Evidence from the marketplace. Econometrica, 72(2), 615-625.

List, J. A., \& Sturm, D. M. (2006). How elections matter: Theory and evidence from environmental policy. The Quarterly Journal of Economics, 121(4), 1249-1281.

Mahul, O., \& Skees, J. (2007). Managing agricultural risk at the country level: The case of index-based livestock insurance in Mongolia. World Bank. Working Paper, No. 4325.

Matsuyama, K. (2007). Credit traps and credit cycles. American Economic Review, 97(1), 503516.

McIntosh, C., Sarris, A., \& Papadopoulos, F. (2013). Productivity, credit, risk, and the demand for weather index insurance in smallholder agriculture in Ethiopia. Agricultural Economics, 44(4-5), 399-417.

Meinke, H., \& Stone, R. C. (2005). Seasonal and inter-annual climate forecasting: The new tool for increasing preparedness to climate variability and change in agricultural planning and operations. Climatic Change, 70(1-2), 221-253.

Meze-Hausken, E., A. Patt \& S. Fritz (2009). Reducing climate risk for micro-insurance providers in Africa: a case study of Ethiopia. Global Environmental Change, 19, 66-73.

Miranda, M., Vedenov, D.V. (2001). Innovations in agricultural and natural disaster insurance. American Journal of Agricultural Economics, 650-655.

Miranda, M.J., Glauber, J.W. (1997). Systemic risk, reinsurance, and the failure of crop insurance markets. American Journal of Agricultural Economics 79, 206-215. 
Mobarak, A. M., \& Rosenzweig, M. (2014). Risk, insurance and wages in general equilibrium (No. w19811). National Bureau of Economic Research.

Mobarak, A.M. and M. Rosenzweig. (2013). Informal risk sharing, index insurance and risk taking in developing countries. American Economic Review, 103: 375-380

Morduch, J. (1994). Poverty and vulnerability. American Economic Review, 84(2), 221-225.

Morduch, J. (1995). Income smoothing and consumption smoothing. The Journal of Economic Perspectives, 9(3), 103-114.

Munshi, Kaivan (2004). "Social Learning in a Heterogeneous Population: Technology Diffusion in the Indian Green Revolution," Journal of Development Economics, 73(1): 185-215.

Neilson, W. S. (2010). A simplified axiomatic approach to ambiguity aversion. Journal of Risk and Uncertainty, 41, 113-124.

Netzer, N. (2009). Evolution of time preferences and attitudes toward risk. American Economic Review, 99(3), 937-55.

Oya, C. (2012). Contract farming in sub-Saharan Africa: A survey of approaches, debates and issues. Journal of Agrarian Change, 12(1), 1-33.

Patt, A., Suarez, P., Hess, U. (2010). How do small-holder farmers understand insurance, and how much do they want it? Evidence from Africa. Global Environmental Change 20, 153-161.

Pitt, M., and S. Khandker. (1998). The Impact of Group-based Credit Programs on Poor Households in Bangladesh: Does the Gender of Participants Matter? Journal of Political Economy, 106(4): 958-996.

Pratt, J.W. (1964). Risk aversion in the small and in the large. Econometrica, 32, 122-136.

Premand, P., \& Vakis, R. (2010). Do shocks affect poverty persistence? Evidence using welfare trajectories from Nicaragua. Well-Being and Social Policy, 6(1), 95-129.

Ravallion, M. (2001). The Mystery of Vanishing Benefits: An Introduction to Impact Evaluation. World Bank Economic Review, 15(1): 115-140.

Ravallion, M. (2007). "Evaluating anti-poverty programs." Handbook of Development Economics 4 (2007): 3787-3846.

Ray, D. (1998). Development Economics. Princeton University Press. 
Renkow, M., Hallstrom, D. G., \& Karanja, D. D. (2004). Rural infrastructure, transactions costs and market participation in Kenya. Journal of Development Economics, 73(1), 349-367.

Rosenzweig, M. R. \& K. I. Wolpin (1993). Credit market constraints, consumption smoothing, and the accumulation of durable production assets in low-income countries: Investments in bullocks in India. Journal of Political Economy, 223-244.

Ross, N., Santos, P., \& Capon, T. (2010). Risk, ambiguity and the adoption of new technologies: Experimental evidence from a developing economy. Unpublished Manuscript.

Sarris, A. (2013). Weather index insurance for agricultural development: Introduction to the special issue. Agricultural Economics, 44: 381-384

Segal, U. (1990). Two-stage lotteries without the reduction axiom. Econometrica: Journal of the Econometric Society, 349-377.

Simon, H. A. (1959). Theories of decision-making in economics and behavioral science. The American economic review, 49(3), 253-283.

Skees, J. R. (2008). Innovations in index insurance for the poor in lower income countries. Agricultural and Resource Economics Review, 37(1), 1-15.

Smith, V. L. (1976). Experimental economics: Induced value theory. The American Economic Review, 66(2), 274-279.

Smith, V. L. (1982). Microeconomic systems as an experimental science. The American Economic Review, 72(5), 923-955.

Staiger, D. \& Stock, J. (1997). Instrumental Variables Regression with Weak Instruments. Econometrica 65(3): 557-586.

Stern, N., \& Taylor, C. (2007). Climate change: Risk, ethics, and the Stern review. Science, 317(5835), 203-204.

Stiglitz, J. E., \& Weiss, A. (1981). Credit rationing in markets with imperfect information. The American Economic Review, 71(3), 393-410.

Storck, H., \& Doppler, W. (1991). Farming systems and farm management practices of smallholders in the Hararghe Highlands. Wissenschaftsverlag Vauk Kiel.

Suri, T. (2011). Selection and comparative advantage in technology adoption. Econometrica, 79(1), 159-209. 
Takahashi, K., Ikegami, M., Sheahan, M., \& Barrett, C. B. (2016). Experimental Evidence on the drivers of index-based livestock insurance demand in southern Ethiopia. World Development, 78, 324-340.

Tedeschi, G. A. (2008). Overcoming Selection Bias in Microcredit Impact Assessments: A Case Study in Peru. Journal of Development Studies, 44(4): 504-518.

Tedeschi, G. A., and D. Karlan. (2010). Cross Sectional Impact: Bias from Dropouts. Perspectives on Global Development and Technology, 9(3-4): 270-291.

Thomas, T., Christiaensen, L., Do, Q. T., \& Trung, L. D. (2010). Natural disasters and household welfare: evidence from Vietnam. The World Bank.

Thornton, P. K., P. G. Jones, G. Alagarswamy, J. Andresen \& M. Herrero (2010). Adapting to climate change: Agricultural system and household impacts in East Africa. Agricultural Systems, 103, 73-82.

Townsend, R. M. (1994). Risk and insurance in village India. Econometrica: Journal of the Econometric Society, 539-591.

Townsend, R. M. (1995). Consumption insurance: An evaluation of risk-bearing systems in lowincome economies. Journal of Economic Perspectives, 9(3), 83-102.

Udry, C. (1994). Risk and insurance in a rural credit market: An empirical investigation in northern Nigeria. The Review of Economic Studies, 61(3), 495-526.

Udry, C. (1995). Risk and saving in Northern Nigeria. The American Economic Review, 85(5), 1287-1300.

Udry, Christopher. (1990). "Credit markets in Northern Nigeria: Credit as insurance in a rural economy." The World Bank Economic Review, 4(3): 251-69.

Vandell, K. D. (1984). Imperfect information, uncertainty, and credit rationing: Comment and extension. The Quarterly Journal of Economics, 99(4), 841-863.

Walker, T. S., \& Ryan, J. G. (1990). Village and household economics in India's semi-arid tropics. Johns Hopkins University Press.

Wantchekon, L., \& Guardado R, J. (2011). Methodology Update: Randomised Controlled Trials, Structural Models and the Study of Politics. Journal of African Economies, 20(4), 653672.

Wilde, L. L. (1981). On the use of laboratory experiments in economics. In Philosophy in Economics (pp. 137-148). Springer, Dordrecht. 
Williamson, S. D. (1986). Costly monitoring, financial intermediation, and equilibrium credit rationing. Journal of Monetary Economics, 18(2), 159-179.

Wooldridge, J. M. (2002). Econometric Analysis of Cross Section and Panel Data. Cambridge, Massachusetts and London, England.

World Bank. (2007). World Bank Report 2008: Agriculture for Development. Washington DC: The World Bank.

Youden, W. J. (1950). Index for rating diagnostic tests. Cancer, 3(1), 32-35.

Zant, W. (2008). Hot Stuff: Index insurance for Indian smallholder pepper growers. World Development, 36(9), 1585-1606.

Zimmerman, F. J., \& Carter, M. R. (2003). Asset smoothing, consumption smoothing and the reproduction of inequality under risk and subsistence constraints. Journal of Development Economics, 71(2), 233-260. 


\section{Summary}

Smallholders in Sub-Sahara African region are the most vulnerable groups in the world. Their capacity to manage risks is especially low due to multiple stressors like drought coupled with their poor asset base. Particularly in Ethiopia, rampant crop losses due to climate change induced drought as well as weather and seasonality-based variations typically characterize the economic loss of farm households. The trend of global climate change has remained devastating and future climatic scenarios are also predicted to be pessimistic as changes in climate and weather patterns are inevitable. In spite of rapid advances in using remote sensing and simulation models, there is no clear picture of how climate variables will change. Hence, in the face of changing climate, the enhanced role of agricultural risk management should be to contribute to mitigation without compromising the role of agriculture for adaptation and food security. As adaptation to climate change is a priority for smallholders and a top agenda for development, demand-driven innovations in agricultural technologies and services are deemed for interventions. Consequently, the last several years have seen the development of innovative instruments for managing weather-related agricultural risks in which global efforts to reduce the impact of climate change on the welfare of households has significantly increased.

One of the recent innovations is the index-based insurance (IBI) which was piloted in different areas in developing countries for managing agricultural risks. The preference for IBI innovations were fueled due to the fact that IBI contracts avoid the twin asymmetric information problems of adverse selection (hidden information) and moral hazard (hidden behavior). Since the indices in IBI innovations were not individual specific, but rely on external parametric measures, neither the insurer nor the insured can manipulate the functioning of IBIs in order to derive unfair

financial advantage. These innovations thus explicitly target and transfer covariate risks to insurers within the contract place and period. Due to these essential characteristics and their potential for adaptation, IBIs have recently attracted the attentions of various scholars and practitioners in the insurance industry. However, almost all pilot experiments undertaken in diversified agro-ecological regions in Africa, Asia and Latin America have met unexpectedly low uptake. While the inherent problem is the prevalence of basis risk in the design of IBI products, various additional caveats were observed in terms of trust in the insurer, financial 
literacy and farmers' ability to understand insurance actuaries as well as the problems of liquidity among smallholders to effect upfront premium payment.

Beyond examining behavioural impediments of IBI adoption like risk and ambiguity aversion which were given less emphasis in previous studies, this thesis was mainly devoted on analysis of the uptake and impact of innovative IBI products that can overcome the longstanding problems like liquidity due to time inconsistency, trust and financial literacy. In Chapter 2, we investigated the role of risk and ambiguity aversion behaviour of smallholders in Ethiopian Rift Valley zone using lab-in-the-field experiments finding that risk aversion behaviour enhances the adoption of IBIs while ambiguity aversion deters the adoption of IBIs among smallholder farmers.

In Chapter 3, we designed an innovative IBI product that postpones the premium payment, and we conducted a randomized control trial in which we exogenously vary between the standard and the innovative IBI as well as between delivery through the conventional statutory cooperative channel and the Iddir-based customary channel. In essence, these innovations help to overcome liquidity constraints and trust problems among the smallholders in deciding to purchase IBI. In addition, since premium postponement potentially invites strategic default, our experiment involves some farmers to sign legal contracts. This experiment first helped us to learn that innovative insurance products that closely address the liquidity constraints of smallholders have significant incremental uptake when channeled through Iddirs which constitute customary social institutions that bear altruistic trust. Second, we learnt that market-based index insurances and predominant indigenous social insurances can function as complementary rural risk management options. IBIs can get an avenue and trustworthiness to reach the community through informal insurances. Informal insurances also benefit from IBIs that manage covariate risks that otherwise erode informal insurances. Integrating the two reinforces (crowds-in) risk-sharing among households.

In Chapter 4 and 5, we systematically measured the impact of IBIs on various categories of household welfare. First, we learned that the adoption of IBIs can help to overcome credit rationing with the implication that credit and IBIs are complementary in the functioning of rural financial markets. Second, the impact evaluation study we reported in Chapter 5 reveals that the adoption of IBI have positive effect on household farm investment. Households who adopted IBI 
have increased investment in high-risk high-return agricultural inputs including chemical fertilizer, improved seed variety as well as pesticide and herbicide. These findings corroborate the theory that adoption of IBIs can have ex-ante (before payout) benefits. In indemnity-based insurance, ex-ante benefits can be negative as insured agents may deliberately produce suboptimal due to moral hazard. But since IBIs are not prone to moral hazard, IBI policy holders can realize such ex-ante benefits from their prudential investment on their insured farms. The implication is that IBI-insured households optimally exert productivity enhancing efforts. Hence, categorizing ex-ante impacts into two as positive (where insured households optimally exert output enhancing efforts) and negative (where insured households may shrink efforts to lower yields and claim payouts), we understood that IBI provides higher ex-ante benefits than indemnity insurance. Thirdly, our welfare impact assessments have shown that the adoption of IBIs increases household consumption. This consumption smoothing role of IBI is the ex-post benefits that insured households enjoy to achieve improved level of welfare compared to their non-insured counter parts.

In general, the results in this dissertation evidence that IBIs are market-based innovative instruments that help to mitigate the adverse effects of climate change and local weather variations on the welfare of households. Though the uptake of the product faces significant challenge due to a host of factors including basis risk, trust, liquidity and understandabaility, uptake can be improved through innovations in product design and/or through innovations in delivery channel. Overcoming the uptake challenges and offering IBIs in a larger scale helps rural households to derive a range of benefits including enhanced access to financial markets, exante prudential investment in productivity enhancing high-risk high-return inputs as well as expost consumption smoothing and shock copying. 


\section{Acknowledgements}

I was benefited from the invaluable contributions of numerous individuals and organizations over the period of my PhD work. First of all, I would like to thank my promotor, Prof. B.W. Lensink, for generously helping me from the start of this study. Robert, your inspiring discussions, encouragement and constant guidance on my $\mathrm{PhD}$ education program has been invaluable. Your contributions have greatly added value on the scientific significance of this thesis. Closely working with you has helped me to be benefited from your expertise to conduct experimental development economics research. At each stage of my $\mathrm{PhD}$ work, I have gained important inputs from your meticulous reviews and critical reflections. Thank you also for your annual socialization and invitation events. My deepest gratitude also goes to my co-promoter Dr. ir. A.P.M. van Asseldonk. Marcel, your insightful reflections, guidance and critical comments have helped me to consider a given item from various perspectives. Thank you very much for all

your inquisitive knowledge and mentorship that contributed a lot in this thesis. In between my heartfelt tribute of misfortune remains with my co-promotor and daily supervisor, Dr. Kees Burger, who unfortunately deceased at the middle of my PhD work. I remain highly indebted for the care and support that Kees made for me till the end of his life.

I would like to thank various individuals who have contributed in different pieces of this study. Prof. Erwin Bulte has helped me to maintain the nice track of my $\mathrm{PhD}$ work at our chair group, and supported me with his special skill of experimental field design. Erwin, I have the great pleasure of working with you. Thank you for all your help to allow me to use the available resource for field work. I have special gratitude for Francesco Cecchi, Anne Winkle, Ana Marr and Shubhashis Gangopadhyay who as co-authors of the different chapters have made their own valuable contributions. I also thank my thesis examining committee members, Prof. Miranda Ruiter, Dr. Cornelis Gardebroek, Prof. Niels Hermes and Prof. Issouf Soumaré, for their interest and willingness to review this thesis and contribute to the public defence. I appreciate your valuable contributions and I am honoured to have you as my thesis examiners. At our Economics Section, I held discussions at different stages and enriched my thesis with Hans-Peter Weikard, Janneke Pieters, Jeroen Klomp, Koen Leuveld, Maarten Voors, Niccolo Meriggi, Rein Haagsma, Jack Peerlings, Cornelis Gardebroek, Nico Heerink, John, Prossy, Aisha and Maria. My PhD study has also been possible with the great support of the administrative staff, all my colleagues 
and students at the Development Economics Group. Thank you all for contributing to our unique family in the academic world with supports and encouragements.

I am highly indebted for the more than 8,500 households in Ethiopia who have taken part in the field experiments and survey rounds of this study. I would really like to thank these individuals for their exhaustive response that they made to satisfy my research inquiry. Using this opportunity, I am thankful for many of the field workers who have helped me for data collection. Implementing agencies for the studies included in this thesis also deserve a great acknowledgement for allowing me to study their projects. I would like to express my gratitude to Oromia Insurance Company (OIC), Japan International Cooperation Agency (JICA), and Ethiopian Ministry of Forest, Environment and Climate Change, for giving me the opportunity, information and support to study their program. I am also thankful a lot to all the staff of the project, especially to Mr. Jin Kimiaki (Ethiopian Country Director of JICA) and OIC staff including Asfaw (CEO), Melkachew (Manager), Habtamu (Crop Insurance Officer) and Getaneh (Livestock Insurance Officer).

Due to resource intensive nature of experimental studies like the studies we have undertaken in this thesis, we have partnered to acquire finance from various agencies that I would like to thank a lot. First, I am profoundly grateful to the Netherlands Organization for International Cooperation in Higher Education (NUFFIC) for granting me the scholarship for my $\mathrm{PhD}$ education. I also sincerely thank the International Initiatives for Impact Evaluation (3ie), the UK Department for International Development (DFID), Economic and Social Research Council (ESRC) and the International Maize and Wheat Improvement Centre (CIMMYT), for the opportunities made available in providing significant amounts of funds for the different phases of the experiments for my $\mathrm{PhD}$ work.

At WUR, I was privileged to be part of a close band of Ethiopian students who always make me feel home. Bayissa, Kassahun, Beshir, Tsion, Gerba, Dereje, Azime, Lemessa, Delelegn, Tariku, Banchayehu, Mohammad, Mohammadamin, Yalemzewud and Tesfaye are my special families and non-forgettable. I have sincere thanks to many of my mentors and colleagues at Haramaya University (HU) who helped me at various stages. The encouragements from Prof. Fikadu Beyene (my local advisor), Prof. Chemeda Fininsa, Prof. Nigussie Dechasa, Prof. Jema Haji, Dr. Jemal Yousuf, Prof. Tessema Zewudu, Dr. Belaineh Legesse and Dr. Mengistu Ketema were 
highly helpful for this work. In addition, Admikew, Robson, Abdeta, Sisay, Kassahun, Dendena and Sonessa at HU have supported me and made my study time more interesting. I am also thankful for Beshir for helping me for the data entry, Kedir for accompanying me at the field for data collection, and Ebrahim Seid (especially for editing the cover of this book). My special gratitude is also to Feyera, Burtkan, Muhammedamin and Juma as well as their children for their special care and support for my family and for me as well. I am so much thankful for my paranymphs, Mark Treurniet and Muhammedamin Hussein for all their support during my preparation for the $\mathrm{PhD}$ defence and to handle invitation of my guests. My lifetime friends including Chemeda, Desalegn, Habtamu, Amsalu, Belete, Ayele and Wakessa deserve a heartfelt word of thanks. I am ever grateful to all of you for everything that we have shared since the early years of our schooling, and will always treasure our great memories.

I have special thanks to my parents, my father, mother and brothers who have shouldered to send me to school and who were unable to see my success today due to the force of nature though they ever wish it. Your legacy will remain memorial with me for all what you did for me to succeed. I am thankful for all my sisters, brothers and relatives at home for all their care and support for me and my family. Finally, I have no enough words to thank my family for their backing during my study. I owe a lot to my wife Alemakef Gedefa for shouldering the whole burden of my family. My PhD work has shared the critical time when my daughters Simera and Heran, and my sons Jinenus and Sihingtan need me the most at home. For this, I bequeath that this book belongs to them as far as it belongs to me. 
Temesgen Keno Belissa

Completed Training and Supervision Plan (TSP)

Wageningen School of Social Sciences (WASS)

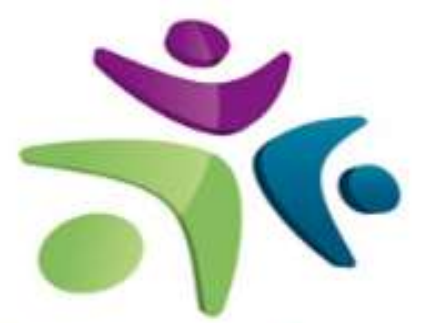

Wageningen School

of Social Sciences

\begin{tabular}{lcc}
\hline Name of the learning activity & $\begin{array}{c}\text { Department/ } \\
\text { Institute }\end{array}$ & Year ECTs* \\
\hline
\end{tabular}

\section{Project related competences}

Advanced Econometrics

Agricultural Economics and Rural Policy $\quad 2014 \quad 6$

(WUR)

Central themes in Development Economics

Development Economics (WUR)

$2014 \quad 6$

Microfinance and Marketing in Developing

Development Economics (WUR)

20146

Countries

General research related competences

Introduction to $\mathrm{PhD}$

WASS (WUR)

$2013 \quad 1$

Information Literacy and End note

WUR library

$2014 \quad 0.6$

Microfinance Experiments: Methods and

University of Groningen

$2014 \quad 2$

application

Research proposal

Development Economics (WUR)

$2014 \quad 6$

\section{Career related competences}

Index-based insurance market development

University of Groningen

$2014 \quad 1$

for pro-poor risk management in Ethiopia

Trust, liquidity constraints and the adoption

of weather insurance: Evidence based on a

Addis Ababa University (Ethiopia)

$2017 \quad 1$

randomized controlled trial in Ethiopia

Trust, liquidity constraints and the adoption

of weather insurance: Evidence based on a

randomized controlled trial in Ethiopia

Stakeholder Workshop, Addis Ababa

$2017 \quad 1$

(Ethiopia)

\footnotetext{
*One credit according to ECTS is on average equivalent to 28 hours of study load

*Additional courses: Advanced Macroeconomics (6 ECTS), Climate Change and Agrarian

Development (2 ECTS) and Rural Economic Analysis (6 ECTS).
} 
The research described in this thesis was financially supported by the Netherlands Organization for International Cooperation in Higher Education (NUFFIC), International Initiatives for Impact Evaluation (3ie), Department for International Development (DFID), Economic and Social Research Council (ESRC) and International Maize and Wheat Improvement Centre (CIMMYT). 
